\title{
Zur Feststellung der Eignung von Schülern für das Fachgymnasium Wirtschaft: Entwicklung und Validierung eines Auswahlinstrumentariums
}

\author{
Dissertation \\ zur Erlangung des Doktorgrades \\ der Mathematisch-Naturwissenschaftlichen Fakultäten \\ der Georg-August-Universität zu Göttingen
}

\author{
vorgelegt von \\ Maike Dittmar \\ aus Naumburg (Saale)
}

Göttingen 2006 
D 7

Referent: $\quad$ Prof. Dr. Marcus Hasselhorn

Korreferent: PD Dr. Dietmar Grube

Tag der mündlichen Prüfung: 18.01.2007 


\section{Vorwort}

Das dieser Dissertation zugrunde liegende Projekt wurde auf Anregung der BBS1 ArnoldiSchule in Göttingen als eine Kooperation mit der Abteilung für Entwicklungspsychologie und Pädagogische Psychologie der Universität Göttingen ins Leben gerufen. Zielsetzung war die Verbesserung der Schülerauswahl sowie die Diagnose des Leistungsniveaus der Schüler am Fachgymnasium Wirtschaft dieser Schule. Die Finanzierung dieses Projektes wurde durch die BBS1 Arnoldi-Schule ermöglicht. Ich danke vor allem der Direktorin Frau Wucherpfennig, den Koordinatoren des Projektes Herrn Tuschling und Herrn Rölleke sowie dem Team der beteiligten Fachlehrerinnen und -lehrer für die gute Zusammenarbeit.

Weiterhin möchte ich mich bei all denen bedanken, die mir darüber hinaus während der Promotionszeit mit Rat und Tat zur Seite standen und die ebenfalls zum Gelingen dieser Arbeit beigetragen haben.

Ein besonderer Dank gilt Herrn Prof. Dr. Marcus Hasselhorn für seine gute Betreuung. Er hat mir ermöglicht, dieses spannende und praxisnahe Projekt durchzuführen und hat mich während dieser Zeit motiviert und mit wertvollen Anregungen unterstützt. Außerdem geht ein Dank an Herrn PD Dr. Dietmar Grube, der sich dazu bereit erklärt hat, die Arbeit als Korreferent zu begutachten. Meinem Kollegen Thorsten Roick danke ich für viele wertvolle fachliche Anregungen und Diskussionen sowie Jörg Behrendt und Thorsten Roick für die mühevolle Arbeit des Lesens der Dissertation und damit verbundene konstruktive Anmerkungen. An dieser Stelle sei auch Alexandra Grote gedankt, die im Rahmen ihrer Diplomarbeit einen wichtigen Beitrag zum Gelingen des Projektes geleistet hat.

Nicht zuletzt geht mein Dank an meine Familie für ihre liebevolle Unterstützung während meiner Promotionszeit. Meinem Freund Dirk danke ich dafür, dass er mir immer hilfreich und aufmunternd zur Seite stand und stets für mich da war. 


\section{Inhaltsverzeichnis}

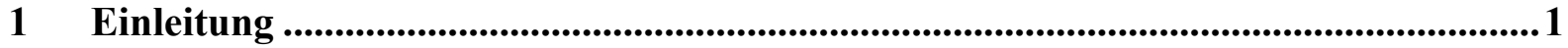

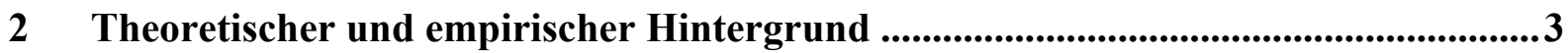

2.1 Allgemeines Prognosemodell nach Krapp (1979) ................................................ 4

2.2 Methoden der Prognose ......................................................................................... 5

2.3 Prognostische Aspekte im Kontext selektiver Laufbahnentscheidungen..................... 7

2.3.1 Merkmale einer selektiven Laufbahnentscheidung …......................................

2.3.2 Prognosemodelle für Vorhersagen im Schulkontext .................................... 10

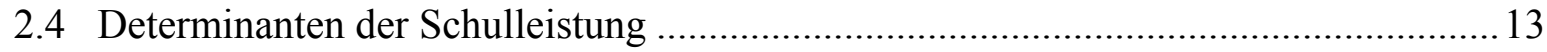

2.4.1 Selbstreguliertes Lernen: Ein Rahmenmodell zum Lernen ............................ 14

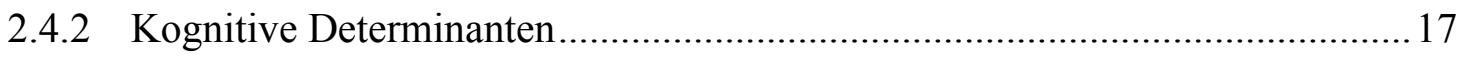

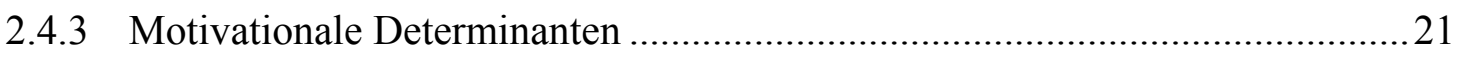

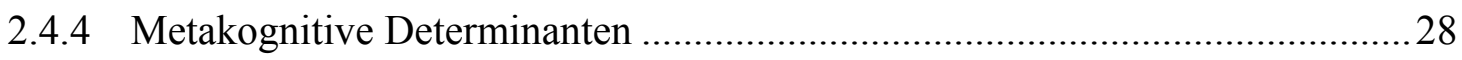

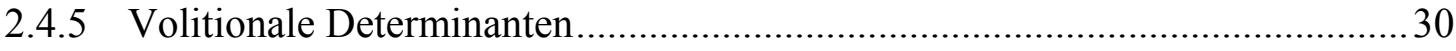

3 Rahmenkonzeption für ein Auswahlverfahren und seine empirisch-basierte

Optimierung .........................................................................................................................................34

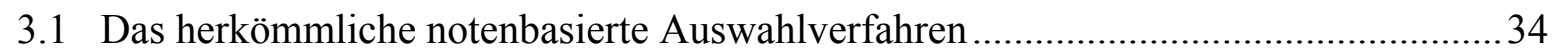

3.2 Vorstellung der Rahmenkonzeption für ein neues Auswahlverfahren........................35

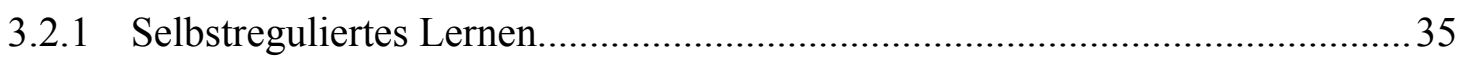

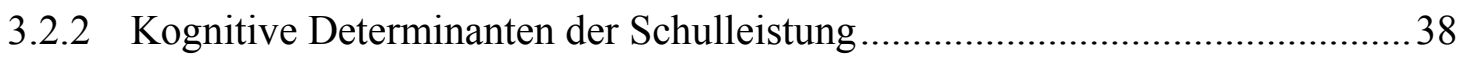

3.2.3 Motivationale Determinanten der Schulleistung .........................................42

3.3 Überblick zur Studie 1: Entwicklung des Diagnoseinstrumentariums ........................46

3.4 Überblick zur Studie 2 (Hauptstudie): Validierung des Diagnoseinstrumentariums ... 46

4 Studie 1: Entwicklung des Diagnoseinstrumentariums ................................................48

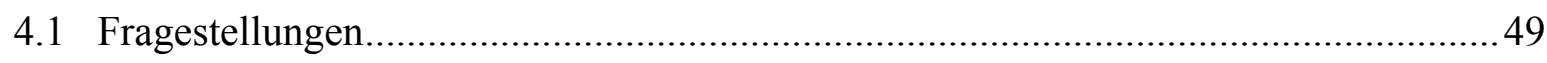

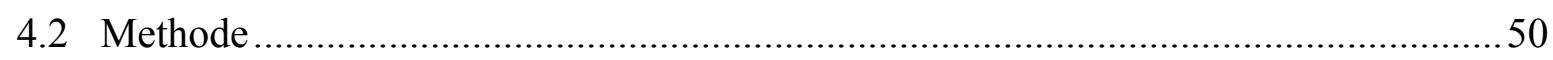

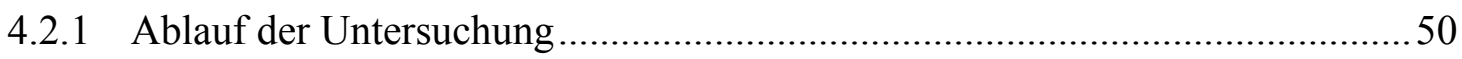

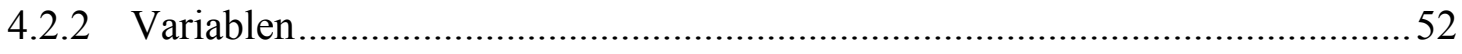

4.2.3 Analysestrategie zur Ermittlung aufgeklärter Kriteriumsvarianz .....................54

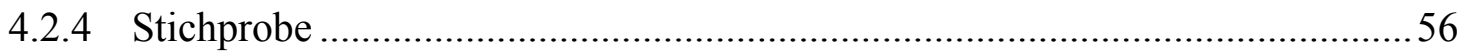

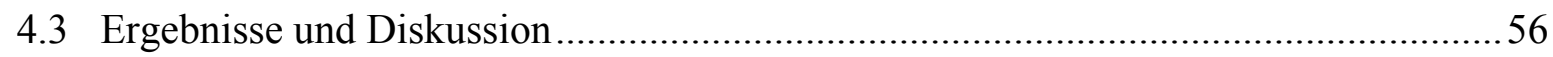

4.3.1 Aufklärung von Kriterienvarianz durch die erhobenen Prädiktorvariablen .....56 
4.3.2 Zur erhöhten Prognosesicherheit des Diagnoseinstrumentariums ....................68

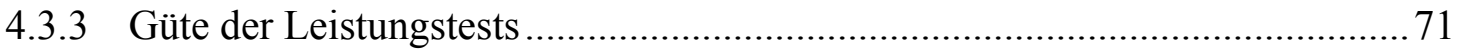

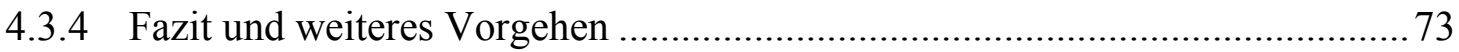

5 Studie 2: Validierung des Diagnoseinstrumentariums ...........................................76

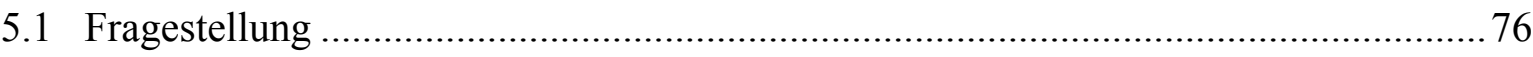

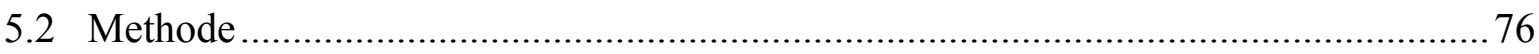

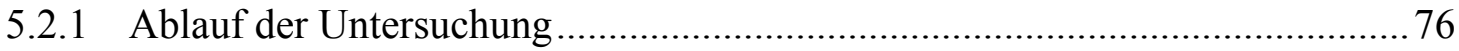

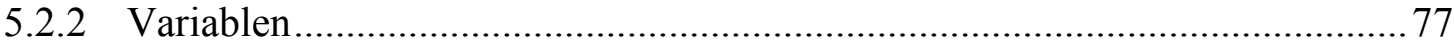

5.2.3 Analysestrategie für die Rangreihenbildung nach dem

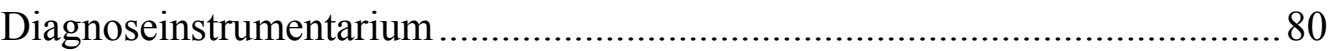

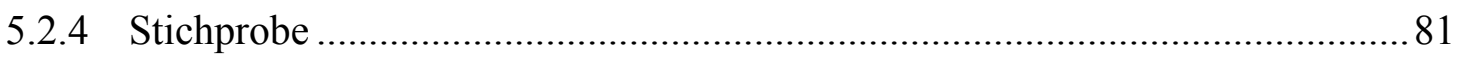

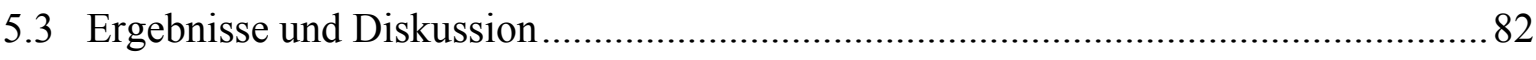

5.3.1 Analysen zur prognostischen Überlegenheit des entwickelten

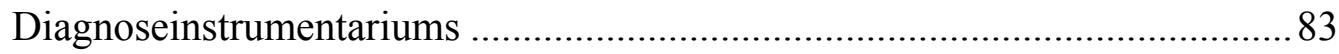

5.3.2 Varianzaufklärung der Validitätskriterien .......................................................93

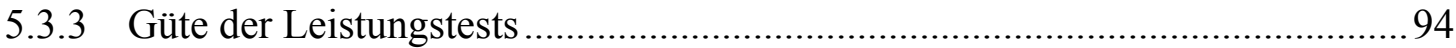

5.3.4 Fazit und weiteres Vorgehen zur Optimierung der Vorhersagegleichungen....96

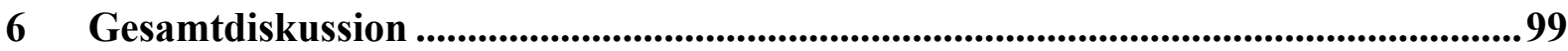

$7 \quad$ Zusammenfassung ...................................................................................................................... 110

Literaturverzeichnis ................................................................................................................... 112

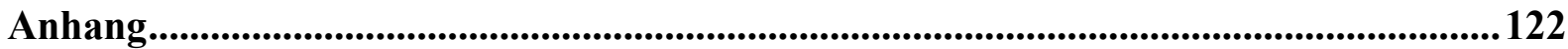




\section{Einleitung}

Selten bestand ein derartig starkes Interesse der Öffentlichkeit an Erziehung und Bildung wie heute. Die TIMSS (Third International Mathematics and Science Study; vgl. Baumert, Bos \& Lehmann, 2000) und die PISA-Studie (Programme for International Student Assessment; vgl. Baumert et al., 2000) haben beide erhebliche Diskrepanzen in der Bildung Jugendlicher zwischen Deutschland und anderen Industrienationen offen gelegt. Seitdem wird der Ruf nach starken Reformimpulsen des deutschen Bildungssystems laut. Selbstverständlich stehen bei entsprechenden Diskussionen neben Universitäten und Fachhochschulen die Schulen mehr denn je im Fokus der Aufmerksamkeit.

Als im Dezember 2001 die Ergebnisse der PISA-Studie veröffentlicht wurden, reagierte die Kultusministerkonferenz unverzüglich mit einem Handlungskatalog (vgl. Kultusministerkonferenz, 2002). In mehreren dort erörterten Punkten zu sieben relevanten Handlungsfeldern wurden Ansätze zur Verbesserung von Bildung und Bildungsangeboten festgelegt. Hieraus ist zum Beispiel der Punkt „Qualitätssicherung von Bildung durch verbindliche Standards und ergebnisorientierte Evaluation“ zu nennen. Mit der Forderung nach einer Verbesserung der Bildungsqualität der Schulen und der Einführung grundlegender einheitlicher Standards verstärkten sich auch Maßnahmen der Standardsetzung und Leistungsevaluation, wie unter anderem die Selektion von Schülern für einen bestimmten Bildungsweg.

Die Gruppe der Fachgymnasien steht hierbei vor einer speziellen Herausforderung, da sie im Vergleich zu allgemein bildenden Gymnasien heterogenere Schülergruppen, bestehend aus Real- sowie Gymnasialschülern, für die 11. Jahrgangsstufe aufnimmt und auf das Abitur vorbereitet. Dies wird beispielsweise durch die relativ hohe Quote von Schulabbrechern an diesen Schulen in der Sekundarstufe II deutlich. Eine zusätzliche Erschwernis stellt das kürzlich eingeführte Zentralabitur dar.

Vor diesem Hintergrund ist das vorliegende Projekt zu sehen. Es wurde auf Initiative einer berufsbildenden Schule ins Leben gerufen, deren vorrangiges Anliegen es war, die bisherige Auswahl der Schüler ${ }^{1}$ für die 11. Jahrgangsstufe ihres Fachgymnasiums Wirtschaft zu verbessern. Aus diesem Grund wurden an Bildungsstandards orientierte Leistungstests der Fächer Deutsch, Mathematik und Englisch entwickelt und in Kombination mit Verfahren zur Erfassung relevanter individueller Merkmale erhoben. Diese Maßnahmen sollen in Hinblick

\footnotetext{
${ }^{1}$ Aus Gründen der besseren Lesbarkeit wird im Folgenden für Personenbezeichnungen die männliche Bezeichnung auch stellvertretend für die weibliche Form verwendet.
} 
auf das von der Kultusministerkonferenz formulierte Handlungsfeld „Qualitätssicherung von Bildung durch verbindliche Standards und ergebnisorientierte Evaluation" langfristig dazu beitragen, mehr Schüler erfolgreich zum Abitur zu führen.

Das Projekt besteht aus zwei Teilstudien. In einer ersten Studie wurde ein Diagnoseinstrumentarium bestehend aus an Bildungsstandards orientierten Leistungstests sowie verschiedenen psychologischen Verfahren der Bereiche kognitive Grundfertigkeiten, Motivation und selbstreguliertes Lernen erstellt und anhand empirischer Befunde auf seine wesentlichen Bestandteile reduziert. Im Rahmen einer zweiten Studie wurde dieses optimierte Instrumentarium in Kombination mit dem herkömmlichen notenbasierten Verfahren des Wirtschaftsgymnasiums zur Auswahl eines neuen 11. Jahrgangs eingesetzt. Das Ziel dieser zweiten Studie war der Vergleich beider Verfahren hinsichtlich der Güte der Schülerauswahl. Anhand der vorliegenden Daten sollen ebenfalls Fragen geklärt werden, die die prognostische Validität der verschiedenen im Diagnoseinstrumentarium enthaltenen Prädiktorvariablen betreffen.

Der theoretische und empirische Hintergrund dieses Themenbereiches ist in Kapitel 2 dargestellt. Im Anschluss daran wird in Kapitel 3 die Rahmenkonzeption des Diagnoseinstrumentariums skizziert sowie dessen empirisch-basierte Optimierung anhand eines strukturellen und zeitlichen Rahmens erläutert. Die Vorstellung der beiden Teilstudien des Gesamtprojektes sowie die Darstellung und Diskussion relevanter Ergebnisse ist in den Kapiteln 4 und $5 \mathrm{zu}$ finden. Abschließend werden in Kapitel 6 die Ergebnisse aus den Teilstudien zusammenfassend und vor dem Hintergrund der Projektziele kritisch diskutiert. Die Arbeit schließt mit einer Zusammenfassung. 


\section{Theoretischer und empirischer Hintergrund}

Die in der Einleitung skizzierte Fragestellung verweist auf einen zentralen Bereich der pädagogischen Psychologie - die Schülerbeurteilung und damit verbundene Diagnose- und Prognoseentscheidungen. Die Erklärung und Vorhersage zukünftigen Verhaltens war und wird ein zentrales Problem der Psychologie, insbesondere der pädagogisch-psychologischen Arbeit bleiben. Da Lernen und Lehren in zielorientierter Form und einem institutionalisierten Kontext stattfindet, interessieren somit auch Aussagen über spätere Schul- oder Studienerfolge sowie Aussagen über die berufliche Entwicklung der Schüler. Dies ist insbesondere relevant für Stationen im schulischen Sozialisationsprozess, in welchen Entscheidungen über zukünftige Schullaufbahnen der Kinder und Jugendlichen anstehen (z.B. Schuleintritt, Versetzung, Übertritt in Schulen der Sekundarstufe, Zulassung zu den verschiedenen Fachhochschulen, Hochschulen). Hierbei handelt es sich auf unterster Ebene um pädagogisch relevante Entscheidungen, die nach verschiedenen Dimensionen eingeteilt werden können. Derartige Entscheidungen können aufgrund eines vorliegenden Prognosemodells getroffen werden, in welchem relevante Größen spezifiziert und in einen kausalen Zusammenhang zueinander gebracht werden (Sauer \& Gamsjäger, 1996). Auf diese relevanten Aspekte soll im folgenden Kapitel näher eingegangen werden.

Eine für den schulischen Kontext sinnvolle und pragmatische Einteilung verschiedener Entscheidungsprozesse aus phänomenologischer Sicht schlagen Krapp und Mandl (1977) vor. Ausgehend von den praktischen Erfahrungen im Schulalltag lassen sich danach im Wesentlichen drei typische Situationen voneinander abgrenzen:

1) die Laufbahnentscheidungen (innere Laufbahnentscheidungen: Klassenwiederholungen, Wechsel zwischen Leistungsgruppen; äußere Laufbahnentscheidungen: alle Arten von Übertrittsentscheidungen),

2) didaktische Differenzierungsentscheidungen und

3) präventiv-therapeutische Interventionsentscheidungen.

Auf Laufbahnentscheidungen wird unter Abschnitt 2.3 genauer eingegangen. Bei didaktischen Differenzierungsentscheidungen geht es darum, möglichst optimale Lernbedingungen und Unterrichtsformen zu schaffen. Die so genannten präventiv-therapeutischen Interventionsentscheidungen beschreiben Entscheidungen, die zur Behandlung auslösender Faktoren von 
Lernstörungen Veränderungen in der familiären und schulischen Umwelt der Schüler bedürfen (Sauer \& Gamsjäger, 1996).

\subsection{Allgemeines Prognosemodell nach Krapp (1979)}

Um Entscheidungen treffen zu können, sind häufig prognostische Aussagen über zukünftiges Verhalten der „Entscheidungsobjekte“ (z.B. Schüler) von Bedeutung. Derartige Aussagen können aus zugrunde liegenden Prognosemodellen abgeleitet werden. Prognosemodelle enthalten theoretische Aussagen über den Zusammenhang von Prädiktoren (vorhersagenden Variablen) und Kriterien (vorherzusagenden Variablen). In Abbildung 1 ist ein solches Modell nach Krapp (1979) in allgemeiner Form dargestellt, welches verschiedenste Arten von wissenschaftlichen Prognosen umfasst. Das Kernstück des Schemas besteht aus zwei Teilen, dem vorhersagenden Teil (Prädiktoren und Prognosetheorie) und dem vorherzusagenden Teil (Kriterien), die durch eine unterbrochene Linie getrennt voneinander dargestellt sind. Dieses Kernstück (Krapp (1979, S. 37) nennt diesen Teil ,prognostisches Argument“) ist in vielen wissenschaftstheoretischen Modellen zur Prognose enthalten. Krapp betont insbesondere einen im Vergleich zu anderen Modellen neuen Aspekt seines Modells, das Konzept der Rahmenbedingungen, welches der Tatsache Rechnung tragen soll, dass Prognosen nicht auf abstrakt-theoretischer Ebene durchgeführt werden können. Prognosen beziehen sich immer auf konkrete Praxisfelder und können deshalb in ein System spezifischer Merkmale der Handlungssituationen eingebettet werden. Hierzu gehören beispielsweise die allgemeine praktische Zielsetzung der Prognose sowie die Auswahl geeigneter theoretischer Sätze aus den verfügbaren Wissensgrundlagen $\left(\mathrm{WG}_{\mathrm{t}}\right)$. Die rationale Vorhersage eines Kriteriums beruht auf theoretischen Annahmen, Hypothesen und Gesetzen, welche in der Prognosetheorie vereint und miteinander in Beziehung gesetzt werden. Generell kann man sagen, dass alle prognostisch relevanten Aussagen in Form sogenannter „Wenn-Dann-Sätze“ vorliegen oder auf Sätzen beruhen, die sich auf diese Struktur zurückführen lassen, wobei die „DannKomponente“ das vorherzusagende Ereignis, also das Kriterium, darstellt. Die „WennKomponente“ wiederum enthält Sachverhalte oder Ereignisse (Prädiktoren), die zur Vorhersage des Kriteriums geeignet sein sollen (vgl. Krapp, 1997). 


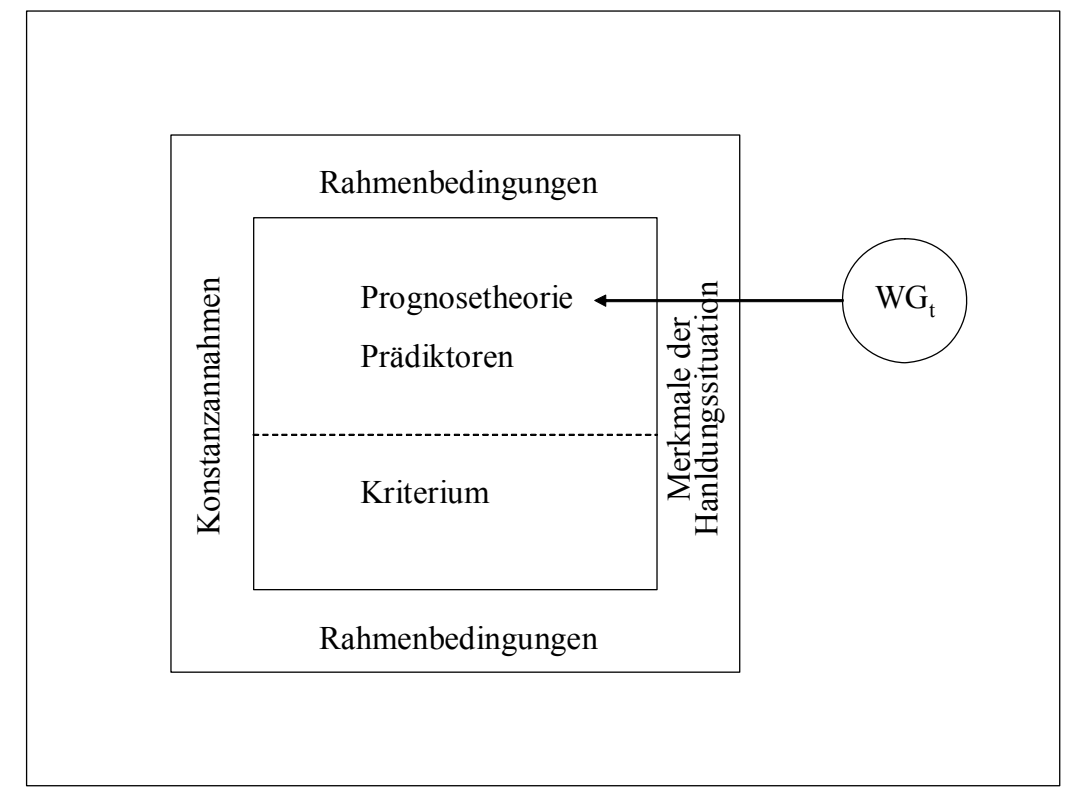

Abbildung 1: Allgemeines Modell der handlungsbezogenen wissenschaftlichen Prognose (nach Krapp, 1979).

\subsection{Methoden der Prognose}

Für die Erstellung von Prognosen wird in den meisten Fällen mit dem Modell der Regressionsanalyse gearbeitet, wobei hier häufig die Modelle der multiplen Regressionsanalyse herangezogen werden. Dieses Verfahren hat den Vorteil, dass mehrere Prädiktoren zur Vorhersage eines Kriteriums herangezogen werden können und dass die einzelnen Prädiktoren mit verschiedenartiger Gewichtung zur Vorhersage des Kriteriums beitragen. Verschiedene andere Verfahren, wie kanonische Korrelationsanalysen, Kovarianzanalysen und auch Pfadanalysen werden als Spezialfälle der multiplen Regression angesehen (Sauer \& Gamsjäger, 1996). Mehrere große Untersuchungen zur Vorhersage von Schulerfolg bedienten sich des Verfahrens der multiplen Regression, wie zum Beispiel die Untersuchungen von Krapp (1973), Gattringer (1980, zitiert nach Sauer \& Gamsjäger, 1996), Kühn (1983) oder Sauer und Gattringer (1986). In diesen Untersuchungen gelang es unter Einbezug unterschiedlicher individueller, familiärer und schulischer Bedingungen sowie kognitiver Prädiktoren mehr als 50\% der späteren Grundschulleistung aufzuklären (Sauer \& Gamsjäger, 1996).

Dabei besaßen in jedem Fall die kognitiven Lernvoraussetzungen bzw. die Intelligenz das stärkste Gewicht zur Erklärung der Schulleistung (Sauer \& Gamsjäger, 1996). Zweifellos hat 
das Modell der multiplen Regression für die Erstellung von Prognosen eine Reihe von Vorteilen. Hierzu gehören zum einen die Möglichkeit der Berücksichtigung mehrerer Prädiktoren zur Vorhersage eines Kriteriums sowie zum anderen der Vorzug, dass sich die Gewichte der einzelnen Variablen zur Bestimmung der Kriteriumsleistung feststellen lassen. Gleichzeitig wird allerdings auch von bestimmten Annahmen ausgegangen, zum Beispiel wird in diesem Modell impliziert, dass sich die Effekte der einzelnen Prädiktorvariablen auf die Kriteriumsvariable additiv verknüpfen. Für den Fall, dass es sich in der Realität nicht so verhält, wird die Vorhersage fehlerbehaftet sein, auch wenn es sich um prognoserelevante Merkmale handelt. Eine weitere Besonderheit ist die zugrunde liegende Annahme, dass die Gewichte der Regressionsgleichung für alle Personen der Population die gleiche Bedeutung haben und somit eine globale Vorhersage getätigt wird. Die Kriteriumswerte aller Personen werden mittels der gleichen Regressionsgleichung vorhergesagt. Hierbei wird der Fakt außer Acht gelassen, dass es auch bei der Größe der Gewichte interindividuelle Unterschiede geben kann (Sauer \& Gamsjäger, 1996).

In Abhängigkeit von der Prognosetheorie und den Rahmenbedingungen kann es sich bei einer Vorhersage um unterschiedlich viele Prädiktoren und Kriterien handeln. Mit Blick auf die Prädiktor- und Kriteriumsvariablen im Prognosemodell kann eine Einteilung nach verschiedenen Prognosetypen vorgenommen werden. Diese Einteilung erfolgt anhand der Zahl der Prädiktor- und Kriteriumsvariablen (Krapp, 1979) und ist in Tabelle 1 dargestellt.

Tabelle 1: Einteilung verschiedener Prognosetypen anhand der Anzahl der Prädiktor- und Kriteriumsvariablen (Krapp, 1979, S. 55).

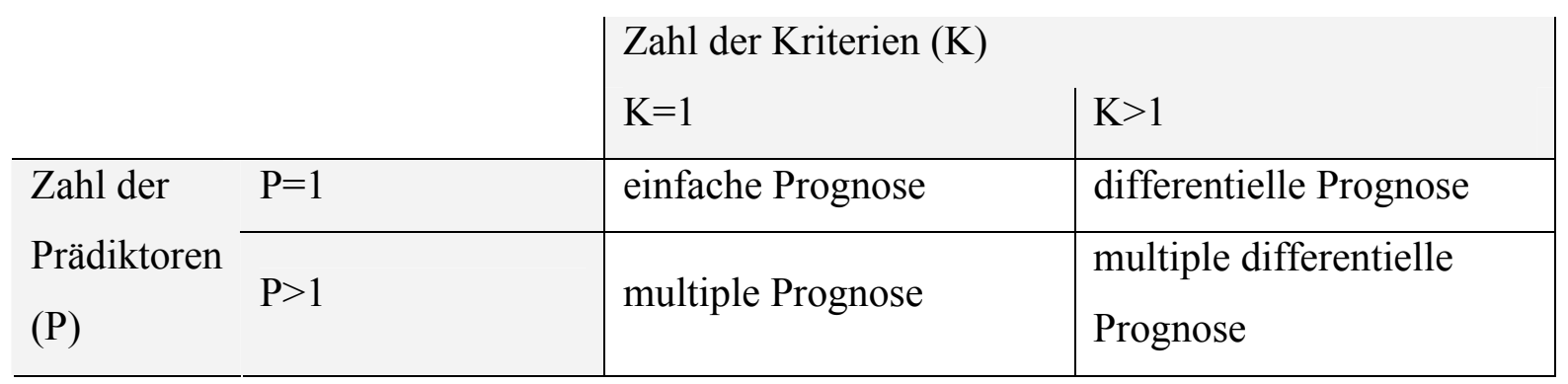




\subsection{Prognostische Aspekte im Kontext selektiver Laufbahnentscheidungen}

Vom phänomenologischen Blickpunkt aus betrachtet, gibt es drei Formen von Prognosen: die selektive Laufbahnentscheidung, die Prognose im Kontext der didaktischen Differenzierungsentscheidung sowie die präventiv-therapeutische Interventionsentscheidung. Bezugnehmend auf das vorliegende Projekt soll im Folgenden näher auf die Prognose im Kontext der selektiven Laufbahnentscheidung eingegangen werden. Formen dieser Prognose sind anhand verschiedener praktischer Beispiele im schulischen Alltag zu beobachten und finden dadurch häufig auch in der breiten Öffentlichkeit Beachtung. Derartige Entscheidungen reichen von Einschulungsentscheidungen, die alle Kinder betreffen über Übertrittsentscheidungen für das Gymnasium bis hin zu Hochschulzulassungsentscheidungen.

\subsubsection{Merkmale einer selektiven Laufbahnentscheidung}

Bei dieser Form der Entscheidung stehen Personen (Schüler, Studenten u.ä.) bzw. Personengruppen im Mittelpunkt. Ein übliches Vorgehen ist die Auswahl einer Teilgruppe aus einer größeren Gruppe, die für eine bestimmte Laufbahn geeignet ist bzw. besonders geeignet erscheint. Die Entscheidung liegt meist bei der aufnehmenden Institution (z.B. Gymnasium) mit dem Ziel der Aufnahme der Bestgeeigneten. Die Handlungsalternativen beinhalten folglich „aufnehmen oder zurückweisen“. Die für die Entscheidung relevanten diagnostischen und prognostischen Informationen enthalten meist Angaben über laufbahnrelevante Persönlichkeitsmerkmale, wie beispielsweise Vorkenntnisse sowie Aussagen über allgemeine und spezielle Fähigkeiten. Im Falle einer Laufbahnentscheidung ist das weitere Vorgehen fest vorgegeben und es wird über die Personenzusammensetzung entschieden. Meist wird ein für alle Individuen gleichermaßen gültiges Entscheidungskriterium herangezogen, beispielsweise der Grad der Erfolgswahrscheinlichkeit in der betreffenden Laufbahn. Offen ist hierbei zunächst noch die inhaltliche Bestimmung derjenigen Bereiche, die in die Bewertung des Erfolges eingehen sollen. Dies kann von den Lernbereichen, die zum Curriculum gehören (z.B. Schulfächer) bis hin zu Bereichen, die nicht direkt zum Aufgabenbereich der Schule gehören (z.B. Motivation, Selbstreguliertes Lernen, Intelligenz) reichen. Die erhobenen prognostischen Daten werden meist schematisch in eine einfache Entscheidungsregel transformiert mit der Funktion, künftiges Leistungsverhalten vorherzusagen. Ein Entscheidungswert innerhalb dieser Regel dient häufig als Grenze zwischen geeigneten und ungeeigneten 
Bewerbern. Dieser Erwartungswert ist ein oft aus mehreren diagnostischen Datenquellen summativ gewonnener Wert, der die allgemeine Fähigkeit zur Bewältigung der Laufbahnanforderungen charakterisieren soll. Zu erwähnen ist hier noch, dass ein Entscheidungsprozess einstufig oder mehrstufig (sequentiell) ablaufen kann. Bei einem mehrstufigen Vorgehen wird sich der Entscheidungsfindung allmählich genähert, indem mit jedem weiteren Schritt die Menge der Bewerber minimiert wird und immer differenziertere diagnostische Verfahrensweisen zur Auswahl eingesetzt werden.

Die laufbahnorientierte Prognose erstreckt sich meist über einen längeren Zeitraum, nämlich über die gesamte Länge der Laufbahndauer bzw. über einen für die Entscheidung relevanten Laufbahnabschnitt. Bei der Entscheidung über die Gymnasialzulassung beispielsweise gilt der Zeitraum von der Zulassungsentscheidung bis zum Abitur als Zeitspanne der Laufbahn mit dem Abitur als Endpunkt dieser Spanne. Eine laufbahnorientierte Prognose soll folglich das Endergebnis einer durch den Treatmentablauf bewirkten Veränderungsreihe vorhersagen. Was in der Zwischenzeit geschieht bzw. auf welche Weise das Endergebnis zustande kommt, interessiert in diesem Zusammenhang nicht. In den meisten Fällen interessiert die Erstellung von individuellen Schätzwerten über den zu erwartenden Ausprägungsgrad eines globalen Gesamtleistungskriteriums. Oft ist es hilfreich, dieses Kriterium auf eine einzige Dimension zu beschränken, da dies die Entscheidungsfindung vereinfacht (z.B. Gesamtschulleistung).

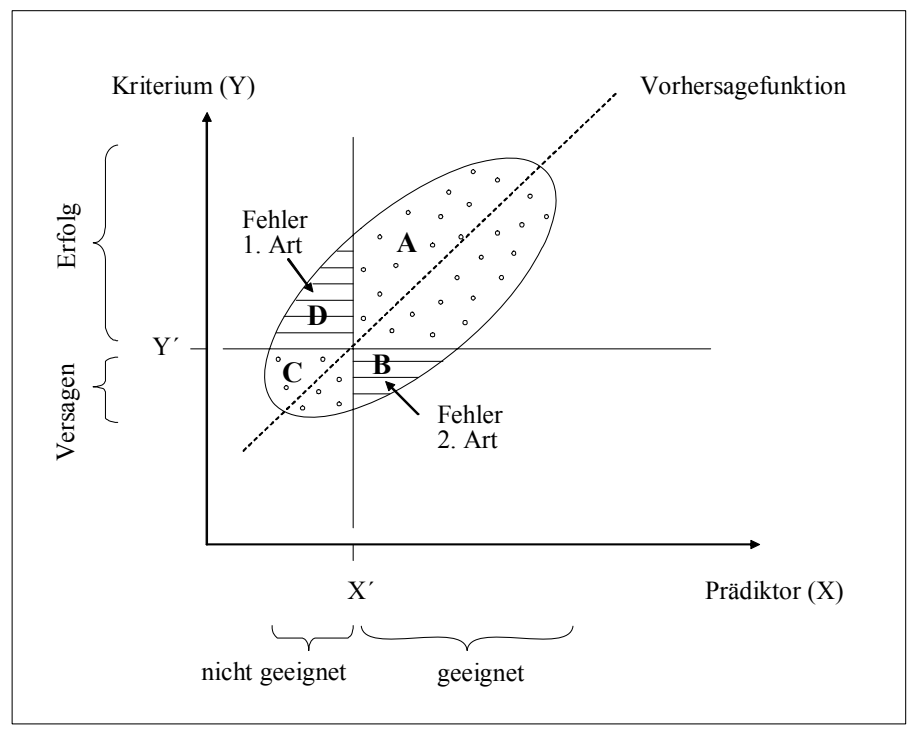

Abbildung 2: Die Beziehung zwischen Prädiktor und Kriterium sowie verschiedene Fehlerund Treffermöglichkeiten dargestellt anhand des Modells der einfachen Selektion (Krapp, 1979, S. 118). 
In Abbildung 2 ist das Prinzip der selektiven Prognose schematisch verdeutlicht. Dieses Prinzip wird hier am einfachsten Fall dargestellt: eine Prädiktordimension $\mathrm{X}$ sagt eine Kriteriumsdimension Y vorher (einfache Prognose). Es wird von einer linearen Beziehung zwischen Prädiktor- und Kriteriumsgrößen ausgegangen, die in Form einer Regressionsgeraden dargestellt ist. In einem Punkteschwarm werden alle einzelnen Werte abgebildet, die Regressionsgerade stellt somit eine Vereinfachung der realen Beziehung dar. Hieran wird deutlich, dass es sich um eine stochastische Beziehung zwischen Prädiktor und Kriterium handelt. Das allgemeine Ziel der Prognose, eine möglichst fehlerfreie Vorhersage des Kriteriums auf der Basis der vorliegenden Prädiktorwerte, kann in dem Maße verwirklicht werden, wie die Streuung der individuellen Punkte um die Regressionsgerade auf ein Minimum reduziert wird. Für selektive Entscheidungen genügt oft die Information, ob ein bestimmter Bewerber auf der Kriteriumsdimension einen bestimmten Mindestwert erreicht oder nicht. In der Abbildung 2 ist dies durch die Trennwerte $\mathrm{X}$ und $\mathrm{Y}$ angezeigt, welche geeignete von ungeeigneten Bewerbern trennen. In der vorliegenden Abbildung kann allerdings noch ein anderer Aspekt der Prognose verdeutlicht werden: die Zahl der Trefferund Fehlerquoten bei selektiven Prognosen. Betrachtet man die Ellipse in Abbildung 2 als Grenze eines Punkteschwarmes, der die Verteilung der Probandendaten im Koordinatensystem repräsentiert, so werden durch die beiden Grenzlinien $X^{\prime}$ und $Y^{\prime}$ vier Gruppen gebildet:

\section{Nach Maßgabe des Prädiktors:}

A: als geeignet klassifizierte und tatsächlich erfolgreiche Probanden (richtig Positive)

B: als geeignet klassifizierte, aber tatsächlich nicht erfolgreiche Probanden (falsch Positive)

C: als ungeeignet klassifizierte und tatsächlich erfolglose Probanden (richtig Negative)

D: als ungeeignet klassifizierte, aber in Wirklichkeit erfolgreiche Probanden (falsch Negative; vgl. Wiggins, 1973 zitiert nach Krapp, 1979).

So handelt es sich bei den Probanden der Gruppen A und C um zutreffende Prognosen (Treffer), bei den Gruppen B und D um unzutreffende Prognosen (Fehler). Hat man für eine große Zahl von Probanden empirische Befunde über prognostische Schätzwerte und unabhängig von diesen Schätzwerten gewonnene Kriteriumsdaten, dann kann man die Qualität der Prognose aufgrund der Verteilung der Bewerber auf die Gruppen A und D zahlenmäßig bestimmen (vgl. Krapp, 1979). 


\subsubsection{Prognosemodelle für Vorhersagen im Schulkontext}

Im Folgenden wird das in den vorigen Abschnitten dargestellte allgemeine Prognosemodell speziell für das Anwendungsgebiet der laufbahnorientierten Prognose inhaltlich angewendet und es werden Aussagen zu den einzelnen Komponenten getroffen. Zunächst wird auf die im Modell explizierten Rahmenbedingungen eingegangen, um anschließend objekttheoretische Aussagen über das Kriterium, die Prädiktoren und die Art der Beziehungen zwischen Kriterium und Prädiktoren vorzunehmen.

\section{Rahmenbedingungen}

Im Modell wurden zwei Gruppen von Rahmenbedingungen unterschieden: (1) Bedingungen, die sich aus dem Handlungszusammenhang ergeben und (2) theorierelevante Konstanzannahmen, die den Anwendungs- bzw. Gültigkeitsbereich der theoretischen Aussagen ermöglichen und gleichzeitig begrenzen. Die selektive Laufbahnentscheidung ist eine institutionelle Entscheidung, die von der jeweiligen Institution nach meist festgelegten Handlungsplänen vorbereitet und durchgeführt wird. Die Art, wie in solchen Institutionen Eignungsdaten erhoben werden können, bestimmt bis zu einem gewissen Grad das Umfeld, aus welchem aufzunehmende Prädiktoren ausgewählt werden. Einschränkende Rahmenbedingungen können sich durch die rechtliche Situation, durch organisatorische Strukturen, Strukturen der Datensicherung sowie zeitliche und finanzielle Grenzen der Datenbeschaffung ergeben. Beispielsweise ist oft die Möglichkeit ausgeschlossen, die individuellen Lernbedingungen innerhalb der familialen Umgebung systematisch zu erfassen, also Daten zu beschaffen, die zwar für die Prognose des Leistungsverhaltens bedeutsam sein könnten, aber dem rechtlichen und moralischen Schutz der Privatsphäre unterliegen. Solche beschränkenden Rahmenbedingungen haben entscheidende Auswirkungen auf die Gestaltung der Prognosetheorie, denn schließlich ist es nicht sinnvoll, in eine praxisnahe Theorie Prädiktoren aufzunehmen, über die der Entscheidungsträger keine Informationen bekommen kann. Eine zweite Form der Rahmenbedingungen sind theoretische Konstanzannahmen. Eine Konstanzannahme ist für alle laufbahnbezogenen Entscheidungen relevant. Es handelt sich um die Überzeugung, dass die für die Laufbahn charakteristischen Lernziele und Lernbedingungen für den Zeitraum der Entscheidung unverändert bleiben. Eine zweite Konstanzannahme betrifft das System leistungsrelevanter Schülermerkmale, der Prädiktoren. Da Laufbahnentscheidungen auf relativ langfristigen Prognosen über zukünftiges 
Leistungsverhalten beruhen, liegt solchen Prognosetheorien die Annahme stabilitätsorientierter Eigenschafts- und Persönlichkeitsmodelle zugrunde.

\section{Kriterium}

Prognosen im Kontext der institutionellen Laufbahnentscheidung sollen individuelles Leistungsverhalten vorhersagen. Als Kriterium wird hier häufig die Schulleistung herangezogen. Vor dem Hintergrund der vorliegenden konkreten Fragestellung der Verbesserung der Schülerauswahl soll an dieser Stelle kurz auf das diesem Projekt zugrunde liegende Konzept von Schulleistung eingegangen werden. Über die Struktur wie auch die Art der verwendeten Kriteriumsmaße gibt es verschiedene Vorstellungen. Diese unterschiedlichen Ansichten über die strukturelle Gestaltung des Kriteriums gehen von einem größtenteils eindimensionalen Merkmal und der Überzeugung aus, dass es eine relativ allgemeine Schulleistungsfähigkeit gibt bis hin zu der Vermutung, dass andere Strukturen relevant sind.

Aufgrund der Tatsache, dass sich Laufbahnentscheidungen vor allem an der prognostischen Information orientieren, ob ein Schüler erfolgreich sein wird oder nicht, ist der Entscheidungsträger meist an globalen Umschreibungen des Leistungsverhaltens interessiert. Die oft angestrebte summative Beurteilung nach den Kriterien Erfolg oder Versagen wird durch eine stärkere Differenzierung des zugrundliegenden Kriteriums, also durch mehrere theoretisch voneinander unabhängige Dimensionen, erschwert. Vor diesem Hintergrund ist eine Bevorzugung von theoretischen Modellen und Methoden, die das Ziel einer weitgehenden Reduktion der Datenfülle bedienen, durchaus nachvollziehbar. In diesem Kontext ist sowohl die traditionelle Notengebung als summative Leistungsbewertung als auch eine objektive Leistungsbeurteilung mit Hilfe von Schulleistungstests vorstellbar. So werden häufig Noten wie auch andere Leistungsindikatoren als Komponenten einer umfassenden Gesamtschulleistung angesehen.

Als Weiteres stellt sich die Frage nach der konkreten Operationalisierung des Kriteriums. Ein wichtiger Gesichtspunkt ist hierbei die Auswahl eines geeigneten Maßstabes. Unter formalen Aspekten können drei Arten von Gütemaßstäben beziehungsweise Normen unterschieden werden: (a) der interindividuelle Maßstab oder die sozialbezogene Norm, (b) der kriteriumsbezogene Maßstab oder die lehrzielorientierte Norm, (c) der intraindividuelle Maßstab oder die personenbezogene Norm. Bei diesen verschiedenen Normen verschiebt sich der Vergleichsmaßstab vom Vergleich mit der sozialen Bezugsgruppe (siehe a), über den Vergleich mit einem konkreten Kriterium (siehe b) bis hin zu einem Vergleich, der sich ausschließlich intern auf das einzelne Individuum bezieht (siehe c). Im Kontext von selektiven 
Laufbahnentscheidungen wird vorwiegend das Konzept der normorientierten Leistungsmessung verwendet. Grundlegendes Ziel der normorientierten Messung ist die möglichst exakte Beschreibung der interindividuellen Leistungsunterschiede (vgl. Krapp, 1979).

Eine weitere Facette des Konstruktes Schulleistung betrifft die Frage danach, ob dieses als situations- und zeitabhängige Verhaltenstendenz oder als ein relativ stabiles Persönlichkeitsmerkmal betrachtet wird. Selektive Laufbahnentscheidungen gehen in Hinblick auf die Bedingungsfaktoren der Schulleistung sowie die vorherzusagende Schulleistung von einer relativen Stabilität der gemessenen Variablen aus (vgl. Krapp, 1979). Für die Berechtigung dieser Sichtweise sprechen empirische Befunde, die belegen, dass interindividuelle Unterschiede zwischen Schülern aus differentialpsychologischer Sicht erstaunlich stabil sind. Diese Unterschiede verringern sich lediglich, wenn Schüler den Schultyp wechseln (vgl. Weinert \& Stefanek, 1997). Aus dieser Perspektive kann Schulleistung als persönliches Merkmal betrachtet werden, welches zwischen Schülern variiert und eine gewisse zeit- und situationsübergreifende Stabilität aufweist (vgl. Heller \& Hany, 2001).

\section{Prädiktoren}

Als zentraler Bereich jeder Prognosetheorie beinhalten die Prädiktoren Aussagen über diejenigen Variablen, die zur Vorhersage des Kriteriums geeignet sein sollen. Auf der Suche nach Variablen, die zur Vorhersage (und/oder Erklärung) von individuellem Leistungsverhalten in der Schule verwendet werden können, sind drei Gruppen von Prädiktoren zu unterscheiden:

1. Faktoren der Schülerpersönlichkeit (individuelle Merkmale, Lernvoraussetzungen, Fähigkeiten, Fertigkeiten)

2. Faktoren der häuslichen Umgebung (Merkmale der familialen Umwelt, z.B. Sozialstatusmerkmale, Erziehungsstil, Familienkonstellation)

3. Faktoren der schulischen Umgebung (Merkmale der Schulorganisation, Ausstattung der Schule, Einflüsse des Lehrers und des Curriculums, Interaktionsbedingungen)

Diese Faktoren sind vor dem Hintergrund gesellschaftlicher und historischer Rahmenbedingungen zu sehen. Im Falle von Laufbahnentscheidungen wird von der Stabilität der für die Prognose relevanten Eigenschaften der Person ausgegangen. 


\subsection{Determinanten der Schulleistung}

In empirischen Arbeiten sowie Studien zu Determinanten von Schulleistung werden verschiedenste Einflussfaktoren diskutiert. Als Determinanten oder auch als Bedingungsfaktoren der Schulleistung verstehen Helmke und Weinert (1997) die ,... Faktoren und Prozesse, [die] die Genese des kollektiven und individuellen Niveaus sowie die Entwicklung der dabei zu beobachtenden intra- und interindividuellen Unterschiede der Schulleistungen 'mitbestimmen'“ (S. 72). Es wird hier also nach Variablen beziehungsweise Faktoren gefragt, die einen Beitrag zur Erklärung intra- und interindividueller Unterschiede der Schulleistungen von Schülern leisten können.

Bei der Wahl der Darstellung beziehungsweise Modellbildung der Bedingungsfaktoren der Schulleistung kann man unterschiedlich große Ausschnitte aus dem Geflecht von Bedingungskonstellationen wählen. Determinanten der Schulleistung reichen von historischen, gesellschaftlichen, kulturellen und wirtschaftlichen Rahmenbedingungen bis hin zu konkreten individuellen Bestimmungsstücken, die Bestandteil der Persönlichkeit des Lerners sind (Helmke \& Weinert, 1997). Für die Entwicklung eines Instrumentariums zur Vorhersage von Schulerfolg soll sich auf die Faktoren der Schülerpersönlichkeit beschränkt werden. Diese Gruppe von Faktoren beinhaltet die für eine Prognose des zukünftigen Schulerfolges aussagekräftigsten Determinanten. Die verschiedenen Faktoren zur Schülerpersönlichkeit können weiter in die Bereiche Kognition, Metakognition, Volition und Motivation unterteilt werden, welche auf verschiedene Art und Weise miteinander in Beziehung gebracht werden können. Vor dem Hintergrund bestehender theoretischer Vorstellungen sollen wichtige und insbesondere für den vorliegenden Rahmen relevante Bereiche und Komponenten dargestellt werden.

Vergleichbar sind beispielsweise die Einteilungen von Helmke und Schrader (2001) sowie von Helmke und Weinert (1997). Die individuellen Determinanten des Schülers werden hier in drei Bereiche eingeteilt. Helmke und Schrader (2001) unterteilen in kognitive (Intelligenz, aufgaben- und bereichsspezifisches Vorwissen), volitionale (Lernstile und Lernstrategien, metakognitive Kompetenzen, Lerngewohnheiten und Arbeitstechniken, Handlungskontrolle) sowie in motivationale Determinanten (Leistungsmotivation, Fähigkeitsselbstkonzept). Helmke und Weinert (1997) unterscheiden ebenfalls zwischen kognitiven (Intelligenz), volitionalen (Lernermerkmale, Arbeitshaltungen, Verhaltensstile, metakognitive Stile, selbstgesteuertes Lernen und Lernstrategien) und motivational-affektiven Determinanten (Leistungsmotivation, Fähigkeitsselbstbild, Prüfungsangst, Interesse, Einstel- 
lung zum Lernen), dabei siedeln sie kognitive und volitionale Determinanten strukturell näher beieinander an.

Bei eingehender Betrachtung wird deutlich, dass manche spezifische Determinanten nur schwer einer dieser globalen Determinantengruppen zuzuordnen sind. Dies betrifft beispielsweise Lernstrategien sowie die metakognitiven Komponenten, die nach diesen Modellen zu dem Bereich der Volition gehören. Beide Konzepte sind auf höherer Ebene den Handlungskontrollprozessen zuzuordnen, da diese wichtige Aufgaben bei der Kontrolle und Überwachung von Lern- und Handlungsprozessen übernehmen. Pekrun und Schiefele (1996) argumentieren allerdings, dass sich Volition und Metakognition nicht hinreichend differenzieren lassen, wenn man allein den Begriff der „,volitionalen Kontrolle“ nach Corno und Kanfer (1993) für den gesamten Bereich der Handlungskontrollprozesse verwendet und diesen nicht weiter unterteilt. Sie differenzieren den globalen Bereich der lernbezogenen Handlungskontrolle weiter auf in „,volitionale Kontrolle“ und „metakognitive Kontrolle“. So können Emotions-, Motivations- und Anstrengungssteuerung als zur „volitionalen Kontrolle“ zugehörig angesehen werden. Planungs-, überwachungs- und lernstrategische Komponenten der Steuerung kognitiver Aspekte des Lernens sind inhaltlich dem Bereich „,metakognitiver Kontrolle“ zuzuordnen.

Nach Pekrun und Schiefele (1996) können also vier globale, ineinander greifende Bereiche motivationaler, kognitiver, volitionaler und metakognitiver Determinanten der Schulleistung unterschieden werden. Das Konzept des selbstregulierten Lernens kann als übergeordnetes Konstrukt aufgefasst werden, welches diese Komponenten beinhaltet. Gelungenes selbstreguliertes und somit selbstständiges Lernen bedient sich kognitiver, metakognitiver, volitionaler und motivationaler Komponenten und gelingt im komplexen Zusammenspiel dieser.

\subsubsection{Selbstreguliertes Lernen: Ein Rahmenmodell zum Lernen}

Zur Einordnung der verschiedenen Determinanten von Schulleistung wird zunächst auf das Konstrukt des selbstregulierten Lernens eingegangen. Nach einer Beschreibung des Konstruktes kann anhand des Modells von Schiefele und Pekrun (1996) der Prozess des selbstregulierten Lernens unter Einbezug von Komponenten der Bereiche Kognition, Motivation, Volition und Metakognition nachvollzogen werden (siehe Abbildung 3).

In den letzten Jahren wird in der Entwicklung der Fähigkeit zum selbstregulierten Lernen - neben der Vermittlung klassischen Fachwissens - eine der Hauptaufgaben der 
Erziehung gesehen. Gleichzeitig handelt es sich beim selbstregulierten Lernen um eine Voraussetzung für den schulischen und außerschulischen Wissenserwerb. Die wachsende Bedeutsamkeit dieses Konstruktes wird auch vor dem Hintergrund der immer rascheren Veränderungen von Wissensbeständen verständlich, wobei das selbstregulierte Lernen mehr und mehr als Schlüsselqualifikation akademischen und beruflichen Erfolges betrachtet wird.

Andererseits wird häufig kritisiert, dass selbstgesteuertes Lernen weder ein präzise definierter wissenschaftlicher Begriff, noch eine einheitlich gebrauchte alltagssprachliche Bezeichnung ist. In dem Umgang mit diesem Konzept wird die Gefahr gesehen, dass es sich zu einem vieldeutigen, ideologieanfälligen Schlagwort entwickeln könnte. Bei einer Durchsicht einschlägiger pädagogischer und psychologischer Studien wird dies anhand der Breite der Definitionen deutlich, deren Variationsbreite teilweise so groß ist, dass kaum noch ein gemeinsamer Begriffskern erkennbar ist (vgl. Weinert, 1982; Kraft, 1999).

So definiert Weinert (1982) selbstreguliertes Lernen als eine Lernform, bei der „... der Handelnde die wesentlichen Entscheidungen, ob, was, wann, wie und woraufhin er lernt, gravierend und folgenreich beeinflussen kann“" (S. 102). Artelt, Demmrich \& Baumert (2001) schlagen folgende konkretere Definition vor: „Lernende, die ihr eigenes Lernen regulieren, sind in der Lage, sich selbstständig Lernziele zu setzen, dem Inhalt und Ziel angemessene Techniken und Strategien auszuwählen und sie auch einzusetzen. Ferner erhalten sie ihre Motivation aufrecht, bewerten die Zielerreichung während und nach Abschluss des Lernprozesses und korrigieren - wenn notwendig - die Lernstrategie“ (S. 271). Schiefele und Pekrun (1996) wiederum definieren selbstreguliertes Lernen in Hinsicht auf die daran beteiligten Bereiche als eine Form des Lernens, „... bei der die Person in Abhängigkeit von der Art ihrer Lernmotivation selbstbestimmt eine oder mehrere Selbststeuerungsmaßnahmen (kognitiver, metakognitiver, volitionaler und verhaltensmäßiger Art) ergreift und den Fortgang des Lernprozesses selbst überwacht“" (S. 258).

Anhand dieser Definitionen wird deutlich, dass selbstreguliertes Lernen ein komplexer Lernvorgang ist, welcher verschiedenste Fähigkeiten und Fertigkeiten einer Person einbezieht. Die Auffassung dieses Konstruktes von Schiefele und Pekrun (1996) ist für den vorliegenden Kontext am besten geeignet. Im Modell zum fremd- und selbstgesteuerten Lernen (siehe Abbildung 3) unterscheiden Schiefele \& Pekrun (1996) zwischen relativ stabilen Lernvoraussetzungen metakognitiver, kognitiver, motivationaler und volitionaler Art, aktuellen Prozessen der Lernsteuerung sowie Produkten des Lernens. Der Lernprozess an sich untergliedert sich in drei Phasen: Planung, Durchführung und Bewertung. Im realen Lernprozess sind sicherlich Abweichungen von der im Modell vorgegebenen Reihenfolge zu 
beobachten. Die Prozesse der Lernsteuerung können sowohl interner als auch externer Steuerung unterliegen, wobei im vorliegenden Modell hauptsächlich Aspekte der internen Steuerung detaillierter dargestellt sind. Die Lernprodukte bestehen nach Auffassung von Schiefele und Pekrun (1996) aus Wissenskomponenten deklarativer und prozeduraler Art (in Abbildung 3 wurde sich auf die Nennung von für schulisches Lernen besonders relevanten Merkmalen der Repräsentation deklarativen Wissens beschränkt).

Das vorliegende Modell bietet einen Rahmen zur Integration motivationaler, metakognitiver, kognitiver und volitionaler Determinanten der Schulleistung, unter der Voraussetzung, dass sich die Qualität und Quantität der Lernresultate (Mikroebene) auch auf die Güte der Schulleistungen (Makroebene) auswirken (vgl. Pekrun \& Schiefele, 1996). So wird davon ausgegangen, dass Schüler, die über günstige selbstregulative Komponenten verfügen, auch bessere Lernresultate und Schulleistungen aufweisen.

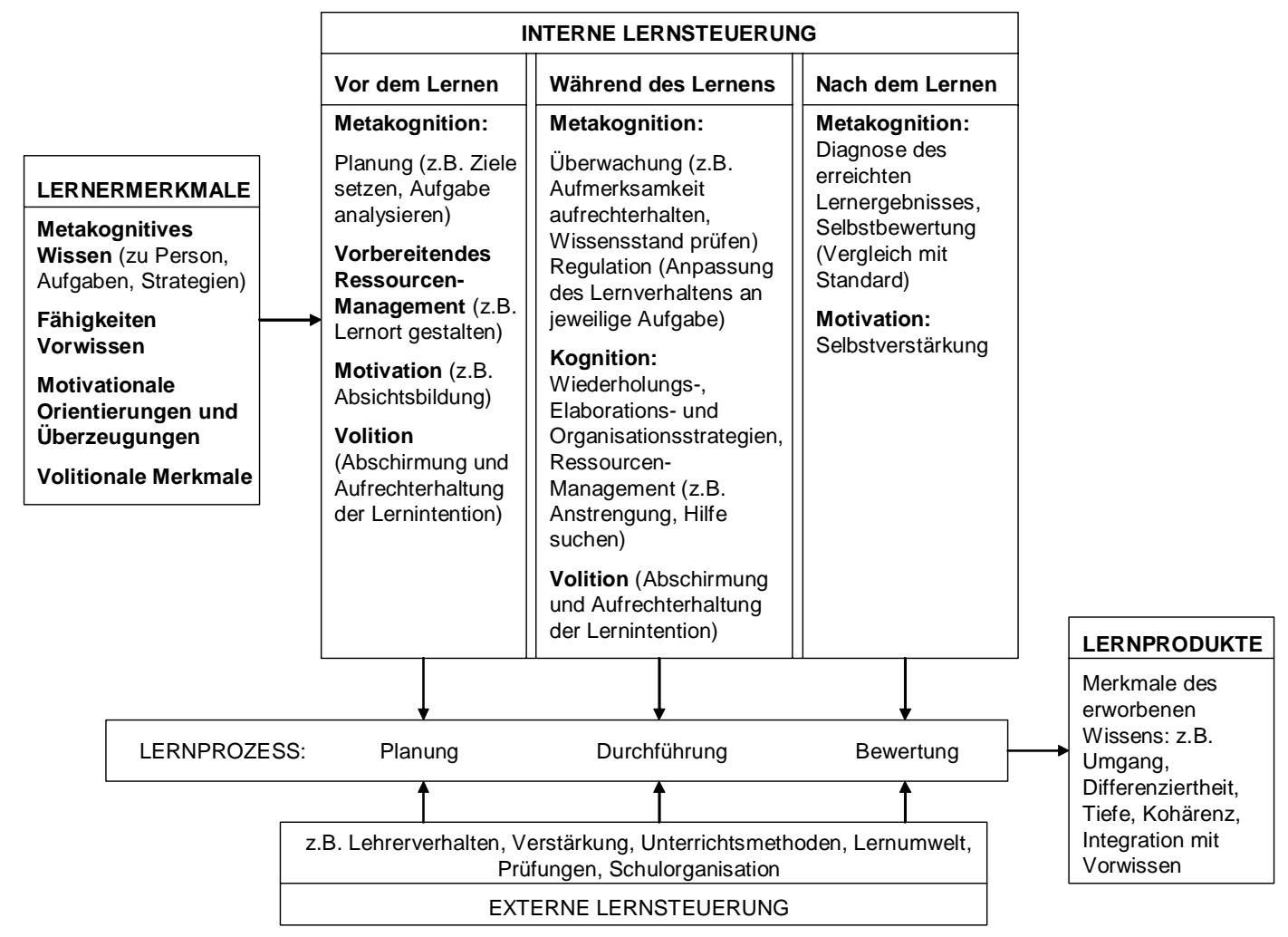

Abbildung 3: Ein Rahmenmodell des fremd- und selbstgesteuerten Lernens zur Verdeutlichung des Prozesses selbstgesteuerten Lernens (vgl. Schiefele \& Pekrun, 1996, S. 271). 


\subsubsection{Kognitive Determinanten}

Die Lernleistung und der Expertiseerwerb werden maßgeblich durch die Intelligenz und das Vorwissen bestimmt. Die prognostische Validität von Intelligenztests sowie des Vorwissens für den Schul- und Studienerfolg ist wiederholt empirisch nachgewiesen worden (vgl. z.B. Heller, Rosemann \& Steffens, 1978; Helmke, 1992a; Kühn, 1983). Des Weiteren ist hier die Konzentrationsfähigkeit zu nennen.

\section{Intelligenz}

Intelligenz bezeichnet im weitesten Sinne die Fähigkeit zum Erkennen von Zusammenhängen und zum Finden optimaler Problemlösungen. Es handelt sich hierbei um eines der am besten untersuchten Persönlichkeitsmerkmale. Obwohl es nach wie vor keine einheitliche Definition von Intelligenz gibt, besteht zumindest Einigkeit hinsichtlich der Art und Beschaffenheit von Aufgaben zur Erfassung der Intelligenz (vgl. Klauer, 2001). Dies wird dadurch verständlich, dass Intelligenz nicht direkt beobachtbar ist, sondern aus verschiedenen Leistungssituationen erschlossen werden muss (vgl. Holling, Preckel \& Vock, 2004).

Wie kann man sich den Zusammenhang zwischen intellektuellen Fähigkeiten und schulischen Leistungen erklären? Es sind zwei kombinierbare Erklärungsansätze denkbar. Erstens sind Intelligentere im Vergleich zu weniger intelligenten Menschen besser in der Lage, sich auf neue Aufgaben einzustellen, effektive Problemlösungsstrategien zu entwickeln und lösungsrelevante Regeln zu erkennen. Zweitens haben intelligente Menschen durch eben diese günstige Voraussetzung in der Vergangenheit mit gewisser Wahrscheinlichkeit intelligenter organisiertes Wissen erworben und können aufgrund dieser bereichsspezifischen Vorkenntnisse leichter darauf aufbauende Lernprozesse bewältigen (vgl. Helmke \& Weinert, 1997).

Stark aggregierte Maße der allgemeinen Intelligenz haben sich als beste Einzelprädiktoren von Schulleistungen erwiesen (vgl. Helmke \& Weinert, 1997). Die Beziehungen zwischen verschiedenen Intelligenzvariablen und Schulleistungskriterien sind in unterschiedlichen Abhandlungen im Überblick dokumentiert (vgl. Heller et al., 1978; Kühn, 1983; Sauer \& Gamsjäger, 1996). Man kann davon ausgehen, dass die mit diversen Intelligenztests erfassten kognitiven Merkmale in der Regel zwischen 25\% und $45 \%$ der Schulleistungsvarianz erklären (vgl. Sauer, 2001). Die Korrelationen mit Schulleistungen liegen bei sprachlichen und naturwissenschaftlichen Fächern bei $r=.30$, für Grundschulnoten sogar bei r=.42 (vgl. Oswald \& Roth, 1978; Roth \& Sauer, 1981). In diesem Zusammenhang 
sei angemerkt, dass sich Intelligenztests zur Prognose individueller Schulleistungsdifferenzen zwar bewährt haben, jedoch wenig Informationen über notwendige Teilfähigkeiten zum Erlernen bestimmter schulischer Prozesse (z.B. Lesen, Schreiben, Rechnen) liefern (vgl. Stevenson et al., 1976). So kann Intelligenz als ein wichtiges, wenn auch nicht hinreichendes Merkmal für die Erklärung von Schulleistungen angesehen werden. Das verdeutlichen zwei Entwicklungen in der Erforschung dieses Zusammenhangs, das bekannte und umstrittene Konzept des sogenannten Over- und Underachievements sowie der Moderatoransatz. Im Konzept des Over- und Underachievements geht es um den Versuch der Erklärung von Abweichungen tatsächlicher Leistungen von den Leistungen, die aufgrund der Intelligenz erwartet werden. Der Moderatoransatz geht davon aus, dass Prädiktoren für verschiedene Gruppen von Personen in Bezug auf ein definiertes Kriterium unterschiedliche Vorhersagewerte haben, d.h. nur in differentieller Weise valide sind. Hier sind verschiedene Drittvariablen denkbar, die den Zusammenhang zwischen Intelligenz und Schulleistung systematisch moderieren. Die Auswahl dieser Variablen wird oft gezielt vom jeweiligen Forschungsinteresse gelenkt. Meist jedoch ist hier der Bereich der sogenannten nichtkognitiven Variablen der Schulleistung vertreten (vgl. Sauer \& Gamsjäger, 1996). Zur Frage der prognostischen Validität von Intelligenzmaßen für die Schulleistung sind zwei Punkte hervorzuheben: zum einen leistet Intelligenz einen signifikanten, aber natürlich nicht hinreichenden Beitrag zur Prognose von Schulleistungen, wobei der Erklärungsanteil bei gleichzeitiger Kontrolle anderer prognoserelevanter Variablen (z.B. Leistungsmotivation) sinkt. Zum anderen ist der Vorhersagewert bei jüngeren Schülern (Grundschülern) größer als bei älteren Schülern (Haupt- und Realschüler, Gymnasiasten), bei denen in zunehmendem Maße auch nicht-kognitive Variablen eine Rolle spielen (vgl. Sauer, 2001). Diese Punkte verweisen stark auf den Bereich nicht-kognitiver Variablen als Ergänzung wichtiger Determinanten, auf die im Anschluss an die Darstellung der kognitiven Determinanten ausführlicher eingegangen wird.

\section{Bereichsspezifisches Vorwissen}

Lange Zeit haben Pädagogische Psychologen und Entwicklungspsychologen die Bedeutung der Vorkenntnisse für das schulische Lernen durch die starke Fixierung auf allgemeine intellektuelle Fähigkeiten unterschätzt (vgl. Helmke \& Weinert, 1997). Der Wissenstand hierzu hat sich jedoch inzwischen grundlegend verändert. Dem bereichsspezifischen Vorwissen kommt gegenüber verbalen und quantitativen Denkfähigkeiten eine vergleichbare Bedeutung als Schulleistungsprädiktor zu. Diese verstärkt sich noch im Verlauf der 
Schulkarriere bzw. bei anwachsendem Komplexitäts- und Schwierigkeitsgrad der Lernaufgabe (vgl. Heller, 1997). Die Erkenntnis, dass das erworbene Wissen eine grundlegende Funktion bei dem Erwerb neuen Wissens hat, wurde im so genannten NovizenExperten-Paradigma weiter vertieft. Vergleicht man Menschen mit ähnlicher Intelligenz, aber einem unterschiedlichen Wissensstand bei der Bearbeitung anspruchsvoller Lern-, Gedächtnis- oder Denkaufgaben aus einem bestimmten Inhaltsgebiet, so übertreffen diejenigen, die über das bessere Wissen verfügen (Experten) die Novizen in praktisch allen Belangen (vgl. Ericsson \& Crutcher, 1990). Dieses für Experten vorteilhafte Wissen umfasst neben deklarativen Kenntnissen auch prozedurale Fertigkeiten, Routinen und Strategien (vgl. Mack, 1995). Experten können für den jeweiligen Gegenstandsbereich auf hierarchisch organisiertes, umfassendes bereichsspezifisches Wissen zurückgreifen (vgl. Glaser, 1991, zitiert nach Spada \& Wichmann, 1996) und beherrschen Lösungsstrategien, die den Möglichkeiten und vor allem Begrenzungen menschlicher Informationsverarbeitung Rechnung tragen (vgl. Simon, 1990). So konnte beispielsweise belegt werden, dass Experten Probleme nach zentralen lösungsorientierten Merkmalen klassifizieren, während Novizen häufig von unwesentlichen, „oberflächlichen“ Eigenschaften ausgehen (vgl. Chi et al., 1981).

Empirische Befunde bestätigen interindividuelle Unterschiede im Vorwissen als einen der stärksten Prädiktoren für Lernleistungen (vgl. Renkl, 1996). In Untersuchungen von Helmke, Schneider und Weinert (1986, zitiert nach Renkl, 1996) zur Prädiktion von Mathematikleistungen bei Hauptschülern konnte dies bestätigt werden. Die Korrelationen zwischen im Jahresabstand erhobenen Vortestleistungen (als Indikator für Vorwissen) und Nachtestleistungen (als Maß für die Lernleistung) lagen zwischen .65 und .74. Weinert (1989) konnte zeigen, dass Vorwissen eine notwendige Bedingung für anspruchsvolle Lernleistung darstellt. So können geringe intellektuelle Fähigkeiten durch gute Vorkenntnisse durchgängig kompensiert werden, während die Möglichkeiten der Kompensation geringer Vorkenntnisse durch hohe intellektuelle Fähigkeiten mit zunehmender Aufgabenschwierigkeit systematisch abnehmen (vgl. Weinert, 1988, zitiert nach Weinert, 1996).

\section{Konzentration}

Konzentration und Aufmerksamkeit sind Konstrukte, die in engem Zusammenhang stehen, die theoretische Abgrenzung beider Konstrukte ist intuitiv zunächst wenig einsichtig. Beiden Konzepten gemeinsam sind eine selektive Komponente sowie die Strukturierung des Wahrnehmungsfeldes (vgl. ten Hoopen, 1996). Konzentration ist in Abgrenzung zur 
Aufmerksamkeit zu verstehen als: „... leistungsbezogene, kontinuierliche und fokussierende Reizselektion ...“ bzw. als Fähigkeit, „... sich bestimmten (aufgaben-)relevanten internen oder externen Reizen selektiv, d.h. unter Abschirmung gegenüber irrelevanten Stimuli, ununterbrochen zuzuwenden und diese schnell und korrekt $\mathrm{zu}$ analysieren“ (S. 6) (Brickenkamp \& Karl, 1986, zitiert nach Brickenkamp, 2002). Konzentration zeichnet sich also gegenüber Aufmerksamkeit durch folgende Kriterien aus: 1. Intentionalität (dies impliziert eine bewusste, intentionale Zuwendung von Aufmerksamkeit), 2. Integration (Integration der selektierten Reize in vorhandene kognitive Strukturen, die intendiert durchgeführt wird), 3. Beanspruchung energetischer Ressourcen (Konzentration wird subjektiv als unterschiedlich anstrengend erlebt), 4. die Abschirmung konkurrierender Reize (das Zielverhalten wird auch gegen konkurrierende Reize und mögliche Ablenkungen abgeschirmt) (vgl. Berg \& Imhof, 2001).

Konzentration und Aufmerksamkeit werden häufig zur Klärung differentialdiagnostischer Fragestellungen herangezogen, beispielsweise, um inter- und intraindividuelle Variationen in verschiedenen Leistungsbereichen zu begründen oder auch um schulische Lernschwierigkeiten eingehender zu beleuchten (vgl. Berg \& Imhof, 2001).

Vom theoretischen Verständnis des Konstruktes ausgehend, erscheint der Zusammenhang der Konzentrationsfähigkeit mit Schulerfolg und Schulleistung plausibel. Empirische Studien belegen niedrige korrelative Zusammenhänge zwischen Konzentration und Schulleistung. Die Rolle der Konzentration als wesentliche Voraussetzung gelungener Lernprozesse ist sicherlich unumstritten. Sie äußert sich stärker im Kontext differentialpsychologischer Fragestellungen, wenn es beispielsweise um die Erklärung von Lernschwierigkeiten geht (vgl. Berg \& Imhof, 2001). In Extremgruppenvergleichen über verschiedene Schultypen und Klassenstufen hinweg (Grund-, Haupt-, Realschule und Gymnasium) konnte gezeigt werden, dass sich leistungsstarke von leistungsschwachen Schülern auch hinsichtlich ihrer Konzentrationsfähigkeit signifikant voneinander unterscheiden. Leistungsstarke Schüler zeigten hier bessere Werte hinsichtlich ihrer Konzentrationsfähigkeit im Vergleich zu leistungsschwächeren Schülern (vgl. Keller, 1993). Schneider und Bös (1985) stellten in ihrer Studie zu Komponenten des Schulerfolges in der Grundschule fest, dass der Beitrag der Konzentration zur Vorhersage der Leistungen für verschiedene Fächer variierte. Sie kommen zu dem Schluss, dass die Effekte der Konzentration auf die Schulleistung als eher gering einzustufen sind. Hellwig (1975) konnte anhand multivariater Analysen einen indirekten Einfluss der Konzentration - gemessen 
mittels des Aufmerksamkeits- und Belastungstests d2 - auf die Schulleistung über die Intelligenz nachweisen.

Anhand dieser Befunde kann ein niedriger positiver Zusammenhang der Konzentrationsfähigkeit mit der Schulleistung angenommen werden. Es ist zu vermuten, dass der Einfluss der Konzentrationsfähigkeit eher in Extremgruppen beziehungsweise in speziellen Settings zum Tragen kommt.

\subsubsection{Motivationale Determinanten}

Durch kognitive Merkmale wird in der Regel $25 \%$ bis $45 \%$ der Schulleistungsvarianz erklärt (vgl. Sauer, 2001). Eine Hinzunahme von motivationalen Lernvoraussetzungen kann diesen Beitrag noch steigern. Trotzdem wurde die Bedeutung nicht-kognitiver Faktoren für die Entwicklung kognitiver Fähigkeiten und für das Leistungsverhalten im Schulkontext lange Zeit unterschätzt. Als zentrale motivationale Komponente ist hier, neben dem Interesse sowie den motivationssteuernden Kognitionen, die Lern- und Leistungsmotivation zu nennen.

\section{Lern- und Leistungsmotivation}

Unter Leistungsmotivation ist „... das Bestreben, die eigene Tüchtigkeit in all jenen Tätigkeiten zu steigern oder möglichst hoch zu halten, in denen man Gütemaßstäbe für verbindlich hält und deren Ausführung deshalb gelingen oder mißlingen kann“ (Lukesch, 1998, S. 343) zu verstehen. Das Leistungsmotiv auf Seiten der Person wird als relativ stabile Verhaltensdisposition verstanden, welche sich als Ergebnis bisheriger Erfahrungen mit Leistungssituationen herausgebildet hat. Dabei kann man Stärke und Gerichtetheit des Leistungsmotivs (bestehend aus den Komponenten: Hoffnung auf Erfolg und Furcht vor Misserfolg) unterscheiden. Das Konzept der Leistungsmotivation ist in allen Situationen von Bedeutung, welche individuelles Verhalten mit Hilfe von Leistungsstandards (durch das Individuum selbst oder durch andere) bewerten und als Erfolg und Misserfolg verstehen (vgl. Sauer \& Gamsjäger, 1996). Hiermit werden schon zwei der grundlegendsten Bestandteile der Theorie der Leistungsmotivation deutlich, Hoffnung auf Erfolg und Furcht vor Misserfolg, die in den Modellen der „McClelland/Atkinson/Heckhausen-Schule“ (Heckhausen, 1989) hervorgehoben werden. In einer Weiterentwicklung der beiden Ansätze von McClelland und Atkinson im Prozessmodell der Motivation von Heckhausen (1972) kommt es durch die Interaktion von Situationen mit Leistungscharakter und der Disposition des Individuums, also seinem Leistungsmotiv, zu einer motivierten Handlung. Merkmale einer Situation, die das 
beim Individuum vorherrschende Leistungsmotiv anregen, können zum Beispiel die Art der Aufgabenstellung betreffen.

Es ist sicherlich verständlich, dass zum Konzept der Leistungsmotivation in Abhängigkeit vom zugrunde liegenden theoretischen Ansatz noch weitere Facetten als zugehörig angesehen werden können (neben den oben aufgeführten Komponenten Hoffnung auf Erfolg und Furcht vor Misserfolg). Zu häufig genannten Dimensionen der Leistungsmotivation gehören unter anderem auch Zielsetzung, Antriebsstärke, Beharrlichkeit und Dominanz (vgl. Schuler \& Prochaska, 2001). Aus der Vielzahl der Ansätze zum Bereich der Lern- und Leistungsmotivation sollen die Konzepte der Zielorientierungen sowie der extrinsischen und intrinsischen Motivation vertiefend dargestellt werden.

Eine in neuerer Zeit von verschiedenen Autoren vorgeschlagene Differenzierung des Konstruktes der Leistungsmotivation ist die Unterscheidung von zwei „Zielorientierungen“ (vgl. z.B. Nicholls, 1989; Dweck, 1986). Hierbei handelt es sich erstens um das Ziel, eigene Fähigkeiten $\mathrm{zu}$ erweitern und zweitens darum, anderen gegenüber hohe Fähigkeit zu demonstrieren bzw. niedrige Fähigkeit zu verbergen. Verschiedene Autoren haben dafür verschiedene Bezeichnungen geprägt. Im Folgenden werden diese Zielorientierungen Bezug nehmend auf die von Dweck (1986) eingeführten Begrifflichkeiten als Lern- und Leistungsziele bezeichnet. Zielorientierungen sind unter anderem hilfreich bei der Erklärung und Vorhersage von Lernverhalten, aber auch von Bewältigungsversuchen nach Misserfolgen. Eine Person mit einer Lernzielorientierung beispielsweise sieht Lern- und Leistungssituationen als Möglichkeit, neue Fertigkeiten zu erwerben bzw. vorhandene Kompetenzen zu erweitern. Eine Person mit einer Leistungszielorientierung wiederum möchte eher ihre Fähigkeiten unter Beweis stellen oder geringe Fähigkeiten verbergen (vgl. Köller \& Schiefele, 2001). Misserfolge werden von Personen mit Lernzielorientierung als Herausforderung angesehen und eine Person reagiert darauf mit bewältigendem Verhalten. Personen mit einer Leistungszielorientierung hingegen zeigen nur dann bewältigendes Verhalten, wenn sie ihre eigenen Fähigkeiten hoch einschätzen (vgl. Köller \& Schiefele, 2001). An diesen Ausführungen wird die unterschiedliche Bedeutung von Zielorientierungen für Motivation und Lernen deutlich.

Unter Lernmotivierung versteht man die momentane Bereitschaft eines Individuums, seine sensorischen, kognitiven und motorischen Funktionen auf die Erreichung eines Lernzieles zu richten und zu koordinieren (vgl. Lukesch, 1998). Bei der Fokussierung auf lernrelevante Motivation, die direkt im Lernalltag beobachtet werden kann, wird eine Unterscheidung von extrinsischer und intrinsischer Motivation vorgenommen (vgl. Pekrun \& 
Schiefele, 1996). Die extrinsische Motivation ist als die Form der Motivation definiert, bei der die Person Handlungsfolgen anstrebt, die außerhalb der eigentlichen Handlung liegen bzw. in einem willkürlichen Zusammenhang mit dieser stehen. Unter intrinsischer Motivation versteht man die Motivation, eine Handlung wegen der unmittelbar mit ihr verbundenen Anreize auszuführen (vgl. Schiefele, 1996; Köller \& Schiefele, 2001). Dementsprechend sind beispielsweise Formen der Kompetenz-, Leistungs- oder sozial orientierten Lernmotivation als extrinsische Motivationsformen aufzufassen (vgl. Pekrun \& Schiefele, 1996). Die Lernzielorientierung weist deutliche Parallelen zum Konzept der intrinsischen Motivation auf. Nach Schiefele (1996) kann die Lernzielorientierung als Streben nach Kompetenzzuwachs definiert werden, was zwar impliziert, dass eine Motivation zum Lernen besteht, aber keinerlei Auskunft über die dahinter liegenden Gründe beinhaltet. Dieses Verhalten kann folglich sowohl intrinsisch als auch extrinsisch motiviert sein. Auch Spinath et al. (2002) betonen die Verwandtschaft der Konstrukte, weisen allerdings aus dem gleichen Grund deutlich darauf hin, dass eine Gleichsetzung von Lernzielen mit intrinsischer Motivation theoretisch nicht begründbar ist. Die Autoren räumen jedoch ein, dass bestimmte Bedingungen, die die Annahme von Lernzielen fördern, auch eher zu intrinsischer Motivation führen, während Bedingungen, die Leistungsziele betonen, eher extrinsische Motivation erzeugen dürften. Generell ist allerdings anzunehmen, dass das Ziel, eine hohe Kompetenz zu erwerben (Lernzielorientierung) Motivation und Lernen im Unterricht fördert und das Ziel, Kompetenz im Unterricht zu zeigen beziehungsweise Inkompetenz zu verbergen Motivation und Lernen im Unterricht beeinträchtigt (vgl. Stiensmeier-Pelster, Balke \& Schlangen, 1996). Grundlegende Annahmen in Dwecks Theorie (1986) konnten in einer Studie von StiensmeierPelster et al. (1996) bestätigt werden.

Der Lern- und Leistungsmotivation wird theoretisch eine große Bedeutsamkeit für die Schulleistung eingeräumt (vgl. Sauer \& Gamsjäger, 1996). Fend (1971) schreibt der Leistungsmotivation als Steuerungssystem des Lernverhaltens einen großen Einfluss auf den Schulerfolg zu und begründet dies damit, dass leistungsmotivierte Schüler:

- $\quad$ eine große Abwehr gegen irrelevante Reize bei Lerntätigkeiten entwickeln,

- mehr Lernaktivität aufbringen,

- eine große Persistenz bei schwierigen Aufgaben zeigen und

- relativ unabhängig gegen äußeren Druck sind.

Anhand der Ergebnisse einer Untersuchung von Keller (1993) konnte belegt werden, dass leistungsstarke Schüler nach eigenen Angaben Freizeitbedürfnisse besser aufschieben können, mehr Vertrauen in die eigene Leistungsfähigkeit haben, konzentrierter lernen sowie den 
Lernprozess besser organisieren. Trotz theoretischer Klarheit sind die empirischen Untersuchungsergebnisse bezüglich des Zusammenhangs zwischen Leistungsmotivation und Schulleistung uneinheitlich (vgl. Sauer \& Gamsjäger, 1996). Ein Streitpunkt stellt die Frage nach dem relativen Stellenwert kognitiver im Vergleich zu motivationalen Variablen für die Aufklärung von Schulleistungsunterschieden dar. Nach einem Vergleich zahlreicher Studien mit unterschiedlichen Stichproben und Erhebungsmethoden kommt Sauer (2001) zu dem Schluss, dass die verschiedenen Kennwerte der Leistungsmotivation zwar einen signifikanten Beitrag zur Aufklärung der Schulleistungsvarianz leisten, jedoch in einem viel geringeren Maße als dies theoretisch erwartet wurde. Helmke wiederum (1992b) hat mit Daten der Münchner Hauptschulstudie belegt, dass der Einfluss nicht-kognitiver Variablen auf die Erklärung interindividueller Unterschiede in den Schulleistungen aufgrund einer Reihe konzeptueller und methodischer Gründe bisher unterschätzt wurde. Er konnte für ältere Schüler (5. und 6. Klasse) zeigen, dass bei der Vorhersage der Mathematikleistung der Prognosewert motivationaler Variablen (49.4\%) nicht wesentlich unter dem der kognitiven Variablen (54.1\%) liegt. Ein anderes Ergebnis der Analyse war allerdings, dass es erhebliche Beträge an konfundierter Varianz gibt, d.h. Varianzanteile, die nicht ausschließlich einem der beiden Prädiktorgruppen zuzuordnen sind (vgl. Helmke, 1992b).

\section{Interesse}

Der enge Zusammenhang der Motivation mit dem Interesse ist insbesondere für intrinsisch motivierte Verhaltensweisen nachvollziehbar. Interesse beschreibt die Wertigkeit, die ein Gegenstandsbereich für eine bestimmte Person repräsentiert (vgl. Krapp, 1992a). Diese Wertigkeit kann auch als kognitive Repräsentation der Verbindungen gefühlsmäßiger Attribute (z.B. Freude) und wertbezogener Attribute (persönliche Bedeutsamkeit) mit dem Interessengegenstand verstanden werden. Diese kognitiven Repräsentationen, oder auch Valenzüberzeugungen genannt, haben intrinsischen Charakter, was bedeutet, dass der Interessengegenstand um seiner selbst willen wertvoll ist (vgl. Schiefele \& Urhahne, 2000). So können intrinsisch motivierte Verhaltensweisen als interessenbestimmte Handlungen aufgefasst werden (vgl. Deci \& Ryan, 1993 ).

In der aktuellen Forschung wird Interesse als mehrdimensionales Konstrukt aufgefasst (vgl. Krapp, 1998). Zur näheren Charakterisierung kann Interesse unter drei Merkmalsaspekten betrachtet werden: einem kognitiven, einem emotionalen und einem wertbezogenen Aspekt. So verfügt eine an einem Gegenstand interessierte Person über mehr oder weniger differenzierte kognitive Schemata über den Bereich dieses Gegenstandes und 
jede weitere Beschäftigung damit führt zu einer Erweiterung der kognitiven Repräsentationen des Interessengebietes. Der emotionale Aspekt beschreibt den Zustand einer emotional anregenden und angenehmen Tönung, der während des Vollzugs der Interessenhandlung auftritt (z.B. Freude, Spannung). Der wertbezogene Aspekt bezeichnet die Tatsache, dass die Interessenhandlung für sich einen eigenständigen Wert darstellt. In neueren Theorien wird davon ausgegangen, dass die individuellen Interessen mit dem Selbstkonzept und der Identität einer Person in Verbindung stehen (Krapp, 1992b).

Mit dem Zusammenhang zwischen der Ausprägung fachspezifischer Interessen und schulischer Leistung hat sich unter anderem die Metaanalyse von Schiefele, Krapp und Schreyer (1993) befasst. Die Autoren trugen in ihrer Metaanalyse Studien des Zeitraumes 1965-1990 zusammen, die sich empirisch mit dem Zusammenhang zwischen Interesse und Leistung beschäftigt haben. Die Korrelationen reichen von .09 bis .67 bei einem Mittelwert von .30. Als Moderatoren zwischen Interesse und Leistung konnten Schiefele et al. (1993) das Schulfach und nur tendenziell das Geschlecht und das Alter sowie die Klassenstufe ausmachen.

\section{Motivationssteuernde Kognitionen}

Im Zusammenhang mit Zielorientierungen und somit Leistungsmotivation stehen auch das akademische Selbstkonzept sowie die schulischen Selbstwirksamkeitserwartungen. Schiefele und Pekrun (1996) ordnen diese den motivationsbezogenen Kognitionen als überdauernde Überzeugungen einer Person zu. Im Folgenden soll kurz näher auf das akademische Selbstkonzept und die schulischen Selbstwirksamkeitserwartungen eingegangen werden.

Als Selbstkonzept versteht man das mentale Modell einer Person über ihre Fähigkeiten und Eigenschaften, bestehend aus gespeicherten kognitiven Repräsentationen über die eigene Person (vgl. Moschner \& Dickhäuser, 2006; Pekrun \& Schiefele, 1996). Der Begriff Selbstkonzept bezeichnet das deklarative Konzept der Kognition einer Person über sich selbst. Diese Einteilung basiert auf dem Vorschlag verschiedener Autoren, eine grundlegende Trennung zwischen Selbstbeschreibungen und Selbstbewertungen vorzunehmen (z.B. Filipp \& Frey, 1987; Helmke, 1992b). Davon zu trennen sind die emotionalen Folgen dieser Repräsentationen, die das globale Selbstwertgefühl als affektiv-evaluatives Konzept konstituieren (vgl. Moschner, 2001). Im Rahmen der Pädagogischen Psychologie wurden Selbstkonzept-Variablen insbesondere im Zusammenhang mit leistungsthematischen Kontexten untersucht (Moschner, 2001). Das akademische Fähigkeitsselbstkonzept (kurz: akademisches Selbstkonzept) kann als die Gesamtheit der kognitiven Repräsentationen 
eigener Fähigkeiten in akademischen Leistungssituationen definiert werden (vgl. Dickhäuser, Schöne, Spinath, \& Stiensmeier-Pelster, 2002).

Über die Struktur, Wirkungsweise und Entstehung des Selbstkonzeptes existieren verschiedene Modelle und Hypothesen (vgl. Moschner, 2001). Während man in früheren Arbeiten häufig von einem globalen Selbstkonzept ausging, besteht heute weitgehend Einigkeit darüber, dass es sich eher um ein differenziertes, vielschichtiges System handelt und dass sich selbstbezogene Kognitionen nicht auf das Selbst als globales Konzept, sondern überwiegend auf spezifische Teilbereiche desselben, beziehen. Ein nach verschiedenen Inhaltsbereichen hierarchisch strukturiertes Modell nehmen Shavelson, Hubner und Stanton (1976) an. Dieses Modell unterscheidet unter anderem zwischen akademischem und nichtakademischem Selbstkonzept. In der überarbeiteten Version von Marsh und Shavelson (1985) werden zwei getrennte Subkonzepte des akademischen Selbstkonzeptes angenommen: das mathematisch orientierte und das verbale Selbstkonzept, denen jeweils noch Faktoren zweiter Ordnung zugrunde liegen (Marsh, 1990). Andere Autoren wiederum verstehen das Selbstkonzept als ein nicht-hierarchisches System von Selbst-Schemata oder gehen von einer Repräsentation selbstbezogener Kognitionen in einem multidimensionalen Raum aus (Moschner, 2001). Auch die Frage nach der Stabilität von Selbstkonzepten hängt eng mit der Einschätzung der Globalität dieser zusammen. Es wird angenommen, dass stark globalisierte Selbstkonzept-Bereiche sowie Selbstkonzept-Facetten mit hoher subjektiver Bedeutsamkeit und Zentralität stabiler sind als bereichsspezifische Facetten des Selbstkonzeptes (vgl. Moschner \& Dickhäuser, 2006). Selbstbezogene Kognitionen können prinzipiell in allen Phasen des Handlungsprozesses bedeutsam werden. So ist das Selbstkonzept im Allgemeinen, aber auch in seinen spezifischeren Facetten hilfreich bei der Verhaltensinitiierung, der Anstrengungsintensität und der Verhaltenspersistenz (vgl. Moschner \& Dickhäuser, 2006).

Neben Indikatoren kognitiver Kompetenzen zählen Selbstkonzepte zu denjenigen Größen, die ebenfalls eng mit schulischen Lernleistungen korrelieren (vgl. Pekrun \& Schiefele, 1996). Der Zusammenhang zwischen Selbstkonzept-Variablen und Leistungen wurde im Rahmen einer Metaanalyse untersucht (vgl. Hansford \& Hattie, 1982). Es wurde eine durchschnittliche Korrelation von $\mathrm{r}=.21$ zwischen Selbstkonzept-Maßen und Leistungsvariablen ermittelt. Unter Berücksichtigung der Studien, in denen lediglich das akademische Selbstkonzept gemessen wurde, stieg die durchschnittliche Korrelation auf $\mathrm{r}=$.42. Für die Adoleszenzphase konnte festgestellt werden, dass der Zusammenhang zwischen hoch generalisierten Fähigkeitseinschätzungen und Schulnoten niedrig ausfällt, während sich fachspezifische und lernbereichsspezifische Fähigkeitseinschätzungen 
stabilisieren (Moschner, 2001). Somit sollte sich die Erfassung der Einschätzung eigener Leistungen und Fähigkeiten möglichst auf das akademische Selbstkonzept oder bereichsspezifische Komponenten desselben beziehen (vgl. Moschner \& Dickhäuser, 2006).

Vom Selbstkonzept ist das Konzept der Selbstwirksamkeitserwartung abzugrenzen. Dieses beinhaltet die Überzeugung einer Person, das zur Erlangung eines Handlungsergebnisses erforderliche Verhalten erfolgreich ausführen zu können und ist somit als Kompetenzerwartung zu verstehen (vgl. Moschner \& Dickhäuser, 2006). Während sich die Selbstwirksamkeit im Leistungsbereich generell auf selbst wahrgenommene Kompetenzen im Hinblick auf die Bewältigung einer Aufgabe beschränkt, sind die Facetten des schulischen Selbstkonzeptes breiter und umfassender (vgl. Köller \& Möller, 2006).

Der Begriff schulische Selbstwirksamkeitserwartung beschreibt die subjektive Gewissheit, neue oder schwierige Anforderungssituationen im schulischen Kontext aufgrund eigener Kompetenz bewältigen zu können. Diese Erwartungen sind insbesondere bei Aufgaben von Bedeutung, die besonderer Anstrengung und Ausdauer bedürfen (vgl. Schwarzer \& Jerusalem, 2002). Es wird angenommen, dass die Einschätzung persönlicher Konsequenzen (Selbstwirksamkeitserwartungen) kognitive, motivationale und affektive Prozesse initiiert, welche wiederum die Umsetzung von Wissen und Fähigkeiten in Handlungen steuern. So kann das Konzept der Selbstwirksamkeitserwartung in ein handlungstheoretisches Erwartungs-Wert-Modell eingebettet werden. Ein solches Modell erklärt Handlungen als Kombination von verschiedenen Vorstellungen bezüglich des zu erwartenden Ergebnisses der Handlung und des Wertes dieses Ergebnisses für die Person, welcher von den antizipierten Folgen des Ergebnisses abhängt (vgl. Preiser, 1998). Vor diesem Hintergrund wird auch die Einordnung der Selbstwirksamkeitserwartung zu den motivationssteuernden Kognitionen, wie es Pekrun und Schiefele (1996) vornehmen, verständlich.

Selbstwirksamkeitserwartungen basieren nach Bandura (1997) sowohl auf der Einschätzung eigener Fähigkeiten, als auch auf der Beurteilung situativer Gegebenheiten. Unter der Berücksichtigung der situativen Erfordernisse schätzt das Individuum ein, inwieweit die eigenen Fähigkeiten in situationsadäquate Handlungen umgesetzt werden können (vgl. Schermer, 2001). Die wahrgenommene Selbstwirksamkeitserwartung ist kein Maß der Fähigkeiten, sondern eine Überzeugung, dass man mit den Fähigkeiten, die man besitzt, etwas Bestimmtes erreichen kann (vgl. Bandura, 1997). Kompetenzerwartungen können hinsichtlich ihrer Stärke, der Allgemeinheit und der Höhe der Anforderungen (dem 
Schwierigkeitsgrad) unterschieden werden. In einer hohen Selbstwirksamkeitserwartung kommt die optimistische Überzeugung einer Person zum Ausdruck, über die notwendigen personalen Ressourcen zur Bewältigung schwieriger Anforderungen zu verfügen (vgl. Satow, 1999). Zahlreiche empirische Untersuchungen belegen, dass hohe Selbstwirksamkeitserwartungen eine Grundbedingung dafür darstellen, dass Anforderungen mit innovativen und kreativen Ideen aufgenommen und mit Ausdauer umgesetzt werden (vgl. Schwarzer \& Jerusalem, 2002).

In vielen Untersuchungen aus dem Bereich der Pädagogischen Psychologie hat sich bestätigt, dass schulische Selbstwirksamkeitserwartungen Leistungsunterschiede erklären und dass deren Vorhersagekraft noch erhöht werden kann, wenn möglichst (fach-)spezifische Selbstwirksamkeitserwartungen herangezogen werden (vgl. Bandura, 1997; Satow \& Schwarzer, 2000; Schunk \& Zimmerman, 1998). Unterschiede in der Selbstwirksamkeit schlugen sich in den Noten der von Jerusalem \& Mittag (1999) untersuchten Fächer Mathematik, Deutsch und Englisch nieder. Bei Schülern mit hoher Selbstwirksamkeit zeigten sich durchgängig bessere Noten als bei Schülern mit niedriger Selbstwirksamkeitserwartung. Diese leistungsmäßigen Vorteile von Schülern mit hoher Selbstwirksamkeit galten für alle untersuchten Jahrgangsstufen (Alter: 13-18 Jahre) sowie für Jungen und für Mädchen. Mittag, Kleine und Jerusalem (2002) beobachteten in ihrer Studie einen generellen Abwärtstrend der Leistung über die Schuljahre in den untersuchten Fächern Mathematik, Deutsch und Englisch. Dieser war bei Schülern und Schülerinnen mit stabilen hohen Selbstwirksamkeitserwartungen nicht zu verzeichnen, was für günstige „Puffereffekte“ hoher Selbstwirksamkeit spricht.

Generell bestätigen diese Befunde einen günstigen Effekt hoher Selbstwirksamkeitserwartungen auf die Leistung.

\subsubsection{Metakognitive Determinanten}

Frühe Definitionen der Metakognition gehen von zwei Komponenten dieser aus, bestehend aus einem deklarativen Wissensaspekt (Wissen über kognitive Funktionen, Produkte und Ziele) und einem exekutiven Kontrollaspekt (Kontrolle der eigenen kognitiven Aktivitäten) (z.B. Flavell, 1976). Diese Einteilung erweist sich für eine angemessene Beschreibung des Gegenstandsbereiches der aktuellen Forschung zur Metakognition jedoch als unvollständig (vgl. Hasselhorn, 2006). Eine weiterführende Untergliederung schlägt Hasselhorn in Anlehnung an Cavanaugh (1989) und Flavell (1976) vor (siehe hierzu auch Tabelle 2). Die ursprüngliche Wissenskomponente (deklarativer Wissensaspekt) wird hier in zwei 
Komponenten unterteilt, in systemisches und epistemisches Wissen. Als eine weitere dritte Kategorie wird die exekutive Metakognition, die mit der zweiten Komponente (exekutiver Kontrollaspekt) des einfacheren Modells korrespondiert, eingeführt. Zwei weitere Kategorien, die allerdings in der bisherigen Metakognitionsforschung wenig Beachtung finden, sind die Sensitivitätskategorie und die Kategorie der metakognitiven Erfahrung (vgl. Hasselhorn, 2006). In Tabelle 2 sind diese fünf Subkategorien der Metakognition noch einmal ausführlicher dargestellt.

Tabelle 2: Integratives Klassifikationsschema der fünf wichtigsten Subsysteme der Metakognition (vgl. Hasselhorn, 1992, S. 42)

1. Systemisches Wissen

a. Wissen über das eigene kognitive System und seine Funktionsgesetze

b. Wissen über Lernanforderungen

c. Wissen über Strategien

2. Epistemisches Wissen

a. Wissen über eigene aktuelle Gedächtniszustände bzw. Lernbereitschaften

b. Wissen über die Inhalte und Grenzen eigenen Wissens

c. Wissen über die Verwendungsmöglichkeiten eigenen Wissens

3. Exekutive Prozesse (Kontrolle)

a. Planung eigener Lernprozesse

b. Überwachung eigener Lernprozesse

c. Steuerung eigener Lernprozesse

4. Sensitivität für die Möglichkeiten kognitiver Aktivitäten

a. Erfahrungswissen

b. Intuition

5. Metakognitive Erfahrungen bezüglich der eigenen kognitiven Aktivität

a. bewusste kognitive Empfindungen

b. bewusste affektive Zustände

Die Vernetzung dieser Systeme im Lernprozess erschwert eine empirische Trennung der einzelnen am Lernen beteiligten Komponenten.

Zur Frage des Zusammenhangs zwischen Metakognition und Leistung kann die Metaanalyse von Schneider (1985) herangezogen werden. Schneider ermittelte einen mittleren Zusammenhang von $r=.41$. Hasselhorn (1992) weist darauf hin, dass metakognitive 
Aspekte notwendig, jedoch nicht hinreichend für intentionales strategisches Lernverhalten sind, welches sich außerdem kognitiver und motivationaler Komponenten bedient (vgl. Short \& Weissberg-Benchell, 1989, zit. n. Hasselhorn, 1992). Zur konkreteren Wirkungsweise von Metakognition auf Leistung weist Weinert (1984) weiterhin darauf hin, dass Komponenten der Metakognition lediglich bei Aufgaben im subjektiven mittleren Schwierigkeitsbereich verbessernd auf Lernleistungen wirken. Auch Vorkenntnisse spielen mitunter für erfolgreiche Lern- und Arbeitsprozesse eine essentielle Rolle und ihr Fehlen kann - trotz des Vorhandenseins von guten metakognitiven Kompetenzen - ausschlaggebend für ungünstige Lernergebnisse sein (vgl. Hasselhorn, 1992).

Zusammenfassend ist zu sagen, dass metakognitive Kompetenzen eine notwendige Bedingung für die Nutzung vorhandener Lernstrategien und somit für Lernerfolg darstellen. Das Konzept der Metakognition ist relativ komplex und schwer von anderen Determinanten erfolgreichen Lernverhaltens $\mathrm{zu}$ trennen. So werden metakognitive Kompetenzen oft im Zusammenspiel mit anderen Determinanten wirksam.

\subsubsection{Volitionale Determinanten}

Die Motivationspsychologie hat lange Zeit das Problem der Umsetzung von Absichten in Handeln vernachlässigt. Die volitionale Steuerung oder Kontrolle dient der Abschirmung eines Vorhabens gegenüber konkurrierenden Einflüssen. Prozesse der volitionalen Steuerung werden dann relevant, wenn die Schwierigkeit, eine Lernabsicht in Verhalten umzusetzen, einen kritischen Wert übersteigt, der Lerner sich aber prinzipiell für fähig hält, die Absicht tatsächlich auszuführen (Kuhl, 1983). Das Ausmaß der volitionalen Steuerung hängt von der Stärke des Ziels. Kuhl hat sechs Mechanismen der volitionalen Kontrolle formuliert:

1) Aufmerksamkeitskontrolle: aktive Lenkung der Aufmerksamkeit auf absichtsrelevante Informationen (selektive Aufmerksamkeit),

2) Enkodierkontrolle: Informationsaspekte, die mit der augenblicklichen Intention in Zusammenhang stehen, werden tiefer verarbeitet,

3) Emotionskontrolle: Beeinflussung der eigenen Gefühlslage zur Sicherung der Handlungsrealisierung,

4) Motivationskontrolle: gegebenenfalls erneuter Einschub eines Motivierungsprozesses,

5) Umweltkontrolle: Veränderung der eigenen Umgebung in einer Weise, die das Durchhalten der aktuellen Absicht fördert, 
6) Misserfolgsbewältigung: beispielsweise über das Abstandnehmen von unerreichbaren Zielen.

Obwohl diese Strategien bewusst eingesetzt werden können, ist in den meisten Fällen davon auszugehen, dass eine automatische unbewusste Anwendung erfolgt (vgl. Heckhausen, 1989). Hinsichtlich des Einsatzes dieser sieben Kontrollstrategien bestehen systematische Unterschiede zwischen Personen, die Kuhl mit den Begriffen Lage- und Handlungsorientierung umschreibt. So haben lageorientierte Personen bei der adäquaten Kontrolle der Aufmerksamkeit, der Motivation und der Emotionen erhebliche Schwierigkeiten.

In den letzten Jahren haben die volitionalen Determinanten der Schulleistung große wissenschaftliche Aufmerksamkeit erfahren (vgl. Corno \& Kanfer, 1993). Die Forschung zum Einfluss volitionaler Komponenten auf die Schulleistung steht allerdings erst am Anfang (vgl. Helmke \& Schrader, 2001). Empirische Befunde zu Zusammenhängen volitionaler Faktoren mit spezifischen Schulleistungen erwiesen sich in zahlreichen Untersuchungen als schwach oder inkonsistent. Allerdings mag dies an den der Komplexität dieser Kontrollprozesse wenig genügenden Versuchsplänen liegen, die dem vielschichtigen Zusammenspiel kognitiver, motivationaler, metakognitiver sowie volitionaler Merkmale und deren Einfluss auf die Schulleistung nicht gerecht werden (vgl. Helmke \& Weinert, 1997).

Auf einige empirische Befunde des Zusammenhangs zwischen volitionalen Determinanten sowie Handlungskontrolle und Leistung soll im Folgenden eingegangen werden. So konnten beispielsweise positive Korrelationen bei Schülern (10-15 Jahre) und Studierenden zwischen Handlungskontrolle und Leistung gefunden werden (Boekaerts, 1994; Volet, 1997). Schiefele und Urhahne (2000) konnten in ihrer Studie zu motivationalen und volitionalen Bedingungen der Studienleistung belegen, dass die Quantität der erbrachten Studienleistungen in einem bestimmten Zeitraum (Zahl der Klausuren) deutlich von der Handlungs- vs. Lageorientierung abhängt. In der konkreten Studie konnte belegt werden, dass handlungsorientierte Personen eine größere Zahl von Leistungssituationen (Prüfungen) aufsuchten. Dies weist darauf hin, dass die Handlungsorientierung dazu beiträgt, eine Lernintention in Verhalten umzusetzen. Hieraus kann man schließen, dass eine hohe Handlungsorientierung Studierende dazu befähigt, Lernabsichten auch unter widrigen Umständen (z.B. Misserfolg) zu verwirklichen. Diese Fähigkeit garantiert vor allem, dass quantitativ mehr geleistet wird. Hinsichtlich der Qualität von Studienleistungen zeigten Boerner, Seeber, Keller und Beinborn (2005) in ihrer Studie mit Studierenden eines 
Fernstudienganges, dass der Einsatz volitionaler Strategien des Zeitmanagements, der Aufmerksamkeits- und der Umweltkontrolle die Qualität der Studienleistungen vorhersagte. Diese empirischen Befunde sowie theoretische Überlegungen weisen auf die Relevanz volitionaler Determinanten für erfolgreiches Lernen und Schulleistungen hin.

Ausgehend von dem Ziel der Verbesserung der Schülerauswahl wurden in diesem Kapitel relevante Determinanten zur Vorhersage von Schulerfolg vorgestellt. Es wurde eine dem vorliegenden Rahmen entsprechende Auswahl der in der Literatur aufgeführten Komponenten getroffen. Dabei sind vier große Variablenkomplexe identifizierbar: kognitive, motivationale, volitionale und metakognitive Determinanten. Im Konzept des Selbstregulierten Lernens können alle vier Bereiche zusammengeführt werden und tragen in ihrem Zusammenspiel zu einem erfolgreichen Lernprozess bei. Diese vier Bereiche wurden mit Blick auf dazugehörige theoretische Konstrukte ausführlich vorgestellt. Eine zentrale Stellung nimmt die Gruppe der kognitiven Determinanten ein. Der Einfluss dieser Variablengruppe ist empirisch vielfach nachgewiesen worden. Von besonderer Relevanz sind das bereichsspezifische Vorwissen und die Intelligenz. Eine untergeordnete Rolle spielt das Konstrukt der Konzentrationsfähigkeit. Zusammenhänge mit der Schulleistung sind hierfür weniger konsistent nachweisbar, doch wird diese Fähigkeit häufig zur Klärung differentialdiagnostischer Fragestellungen herangezogen, um beispielsweise interindividuelle Variationen im Leistungsverhalten zu erklären.

Deutlichere Zusammenhänge zur Schulleistung weisen das Vorwissen und die Intelligenz auf. Die essentielle Rolle des Vorwissens verstärkt sich noch im Laufe der Schulzeit beziehungsweise bei anwachsender Komplexität der Lernaufgabe. Beispielsweise besteht die Möglichkeit einer Kompensation geringer intellektueller Fähigkeiten durch gute Vorkenntnisse. So ist dem Vorwissen, gerade in höheren Schulklassen, eine tragende Rolle zur Bewältigung erfolgreichen Lernens zuzuschreiben. Die Rolle der Intelligenz für Schulleistung ist ebenfalls wichtig, diese nimmt bei älteren Schülern im Vergleich zu jüngeren Schülern in ihrer Prognosekraft ab.

Die Existenz guter kognitiver Fähigkeiten kann unter bestimmten Voraussetzungen erst im Zusammenspiel mit anderen Merkmalen entfaltet werden. An dieser Stelle bedarf es eines Einbezugs nicht kognitiver Merkmale, wie motivationaler Determinanten der Schulleistung sowie volitionaler und metakognitiver Prozesse. Die motivationalen Komponenten spielen im Vergleich zu den kognitiven Determinanten eine untergeordnete Rolle, doch sollte ihre Bedeutung für erfolgreiches Lernen, gerade bei älteren Schülern, nicht unterschätzt werden. 
Diese Komponenten stellen eine wichtige Ergänzung zu den kognitiven Determinanten dar. Ein gelungener Lernprozess beinhaltet allerdings ebenso volitionale und metakognitive Komponenten. Zur Erfassung wichtiger Voraussetzungen erfolgreicher Lernprozesse und somit selbstregulierten Lernens ist es sinnvoll, Variablen aus allen vier Bereichen $\mathrm{zu}$ berücksichtigen. Ein Schwerpunkt sollte dabei auf der Erhebung der kognitiven Determinanten liegen, welche durch die aufgeführten Komponenten aus dem Bereich motivationaler Determinanten ergänzt werden. Der Einbezug von Variablen zur volitionalen und metakognitiven Kontrolle stellt eine als wertvolle Ergänzung der bisher erwähnten Determinanten dar. Die Auswahl geeigneter Verfahren aus diesen Bereichen wird im folgenden Kapitel ausführlich dargestellt. 


\section{Rahmenkonzeption für ein Auswahlverfah- ren und seine empirisch-basierte Optimierung}

In dem vorliegenden Projekt geht es darum, die Auswahl der Schüler für die 11. Klassenstufe des Wirtschaftsgymnasiums aufgrund reliabler und valider Analysemethoden zu verbessern. Ausgangspunkt dieser Fragestellungen ist das bisher etablierte Auswahlverfahren, welches auf den Noten der Herkunftsschulen der Schüler basiert. Aufbauend auf der Vorstellung dieses Vorgehens, wird in einem nächsten Schritt die Auswahl geeigneter Komponenten für das neue Diagnoseinstrumentarium dargestellt sowie Schritte für deren empirisch-basierte Optimierung skizziert.

\subsection{Das herkömmliche notenbasierte Auswahlverfahren}

Ausgangspunkt bildeten hierbei die Endjahresnoten der 10. Klasse für die Fächer Deutsch, Englisch und Mathematik der Herkunftsschulen. Hieraus wurde für jeden Schüler ein Summenwert gebildet, der weitere relevante Kriterien, die Beurteilung des Arbeits- und Sozialverhaltens der Schüler, die Schulform der abgebenden Schule sowie gegebenenfalls eine Empfehlung des Klassenlehrers, berücksichtigte. So wurden beispielsweise zwei Bewertungspunkte von der Notensumme abgezogen, wenn die Noten auf einem Gymnasium erhalten wurden, zwei Bewertungspunkte wurden addiert, wenn es sich bei der abgebenden Schule um eine Hauptschule handelte. Die Beurteilungen des Arbeits- und Sozialverhaltens seitens der abgebenden Schule wurden vor der Summenbildung in Bewertungspunkte (von -1 bis +2) überführt. Der gebildete Summenwert konnte durch einen weiteren Punkt (-1 bzw. +1) verändert werden, wenn eine Empfehlung oder eine kritische Einschätzung der Eignung eines Schülers für die gymnasiale Oberstufe seitens des ehemaligen Klassenlehrers vorlag. Die Berechnung von Summenwerten aus diesen Informationen ermöglichte die Einstufung aller Bewerber in eine Rangfolge. Schüler mit den niedrigsten Punktwerten wurden bei der Auswahl bevorzugt. Bis zu einem Gesamtpunktwert von 9 wurde eine Zusage erteilt.

Die Verteilung der Plätze orientierte sich neben dem Punktwert in der Rangliste auch an einigen anderen Kriterien. So wurde ein Zehntel der vorhandenen Plätze Bewerbern zugesprochen, deren Ablehnung eine außergewöhnliche Härte darstellen würde. Von den 
verbleibenden Plätzen sollten bis zu 40 an die Schüler vergeben werden, die in einem früheren Schuljahr wegen fehlender Plätze nicht aufgenommen werden konnten. In diesem Fall legte die jeweilige Dauer der Wartezeit die Rangfolge der Bewerber fest. Für den Fall gleich langer Wartezeiten und für die übrigen Plätze sollte eine Vergabe nach Eignung und Leistung erfolgen. Über diesen Weg wurden in der Regel zahlenmäßig die meisten Schüler ausgewählt. Die restlichen Plätze wurden an Schüler vergeben, die trotz eines höheren Punktwertes noch als bedingt geeignet beurteilt werden konnten.

\subsection{Vorstellung der Rahmenkonzeption für ein neues Auswahlverfahren}

Im vorigen Kapitel wurden relevante Determinanten der Schulleistung dargestellt und vor dem Hintergrund ihres Zusammenhangs zum Schulerfolg betrachtet. Ausgehend von diesen theoretischen Überlegungen wird in den folgenden Abschnitten die konkrete Auswahl von Verfahren für ein Diagnoseinstrumentarium dargestellt. Zunächst werden Verfahren zum Bereich des Selbstregulierten Lernens vorgestellt. Diese beinhalten Komponenten der Bereiche Motivation, Volition und Metakognition. Anschließend werden die zu dem Bereich Kognition und Motivation ausgewählten Verfahren aufgeführt. Die im Folgenden vorgestellten Fragebögen sind im Anhang zu finden.

\subsubsection{Selbstreguliertes Lernen}

Es wurden zwei Fragebögen ausgewählt: zum einen der Fragebogen zum Selbstregulierten Lernen aus der PISA-Studie (vgl. Baumert et al., 2000), zum anderen die Kurzform des Selbststeuerungsinventars von Rheinberg und Wendland (SSI-K-32) (2003).

Der Fragebogen zum Selbstregulierten Lernen aus der Pisa-Studie umfasst verschiedene Bereiche der Determinanten von Schulerfolg. Betrachtet man selbstreguliertes Lernen tatsächlich als den verschiedenen Bereichen zu Determinanten der Schulleistung übergeordnet (kognitiv, motivational, metakognitiv und volitional), dann ist es durchaus sinnvoll, diese Bereiche in einem Fragebogen zu verknüpfen. Die Autoren der PISA-Studie gehen davon aus, dass das Vorhandensein der in diesem Fragebogen enthaltenen Merkmale bei Schülerinnen und Schülern die Wahrscheinlichkeit erhöht, positive Lernresultate zu erzielen. In dem Fragebogen wurden 11 solcher Merkmale in 11 Skalen erfasst, es wurden 9 
Skalen daraus für das Diagnoseinstrumentarium übernommen. Dieser Bogen umfasst vier große Bereiche, in denen sich diese 9 Skalen verteilen:

- Lernstrategien: Wiederholungsstrategien, Elaborationsstrategien, Kontrollstrategien),

- Motivationale Präferenzen: Instrumentelle Motivation, Interesse (Lesen), Interesse (Mathematik),

- Selbstbezogene Kognitionen: Kontrollerwartungen, Selbstwirksamkeitserwartungen,

- Handlungskontrolle: Anstrengung und Ausdauer beim Lernen.

Die Items dieser Skalen sind im Original auf einer vierstufigen Ratingskala hinsichtlich der Auftretenshäufigkeit von „fast nie“ bis „fast immer“ bzw. hinsichtlich des Grades der

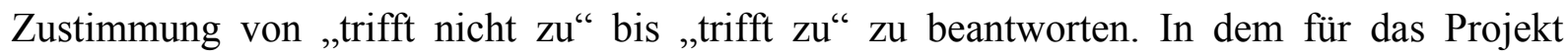
erstellten Fragebogen wurden die Items in eine sechsstufige Ratingskala von „Trifft gar nicht $z u^{\prime \prime}$ bis „Trifft vollständig zu“ überführt. Die Veränderung der Anzahl der Stufen wurde vorgenommen, um die verschiedenen Ursprungsfragebögen und -skalen in möglichst wenigen Fragebögen zusammenfassen zu können und Unsicherheiten bei den Schülern zu vermeiden. Es wurde davon ausgegangen, dass die Erhöhung der Stufen der Ratingskala keinen erheblichen Einfluss auf die Gütemerkmale der Ursprungsskala hat.

Im Folgenden werden die verschiedenen Skalen des Fragebogens zum Selbstregulierten Lernen kurz dargestellt.

Ein zentraler Bereich des selbstregulierten Lernens ist der Themenbereich Lernstrategien/Lernstile. Die Lernstrategien sind größtenteils im Bereich der kognitiven und metakognitiven Regulation anzusiedeln, doch sind auch motivationale und volitionale Mechanismen für eine effiziente Nutzung der Lernstrategien von Bedeutung. In der Literatur zu Lernstategien bestehen allerdings in der Definition dieser erhebliche Unterschiede (vgl. Bjorklund \& Harnishfeger, 1990), beispielsweise hinsichtlich der Bewusstheit der jeweiligen Handlung oder auch bezüglich der angenommenen Stabilität bzw. Komplexität von Lernstrategien. Oft wird eine grobe Einteilung in kognitive Strategien, metakognitive Strategien und Strategien des Ressourcenmanagements vorgenommen (vgl. z.B. Friedrich, 1995). Zu den kognitiven Strategien zählen dabei Memorierstrategien (Einprägen durch wiederholtes lautes Vorlesen, Auswendiglernen), Elaborationsstrategien (Konstruktion, Integration, Transfer) und Transformationsstrategien (Übertragung der Informationen auf ein anderes Medium). Zu den sogenannten metakognitiven Strategien zählen Planung (Setzen von Lernzielen, Formulieren von Kontrollfragen), Überwachung (wiederholtes Prüfen, ob das Gelesene verstanden wurde) 
und Regulation (Anpassung der Lerntätigkeit an Aufgaben, z.B. Lesegeschwindigkeit herabsenken). Aus dem Pisa-Fragebogen zu selbstreguliertem Lernen wurden zwei kognitive Strategien, Wiederholungsstrategien (4 Items), Elaborationsstrategien (4 Items), und eine metakognitive Strategieskala, Kontrollstrategien (5 Items), entnommen.

Eine ebenfalls zentrale Voraussetzung für selbstreguliertes Lernen ist das Vorhandensein einer hinreichenden (Lern-)Motivation. Aus dem Pisa-Fragebogen für selbstreguliertes Lernen wurden drei Skalen aus dem Bereich motivationaler Präferenzen entnommen. Von diesen motivationalen Komponenten des selbstregulierten Lernens wird angenommen, dass sie die Investition von Zeit und mentaler Energie für Lernzwecke regulieren und die Auswahl von Lernstrategien beeinflussen. Wie in Kapitel 2 deutlich wurde, können Interessen auch als motivationale Tendenzen verstanden werden. Sie weisen einen engen Bezug zur intrinsischen Lernmotivation auf. Interesse hat einen Einfluss auf die Kontinuität und Intensität des Engagements in Lernsituationen und auf die erreichte Verstehenstiefe. Deshalb wurden zum Bereich Interesse die Skalen, Interesse (Lesen) und Interesse (Mathematik), übernommen. Des Weiteren wurde die Skala Instrumentelle Motivation in das Diagnoseinstrumentarium aufgenommen. Diese Skala erfasst mit drei Items die extrinsische Motivation, die auf den Zielen beruht, eigene Berufschancen zu verbessern sowie die Aussicht auf eine gute Arbeitsstelle zu erhöhen, also insgesamt berufliche Ziele zu verwirklichen. Diese Skala wurde ausgewählt, da sie sich als wichtiger Prädiktor für Fachleistungen erwiesen hat (vgl. Eccles \& Wigfield, 1995; Wigfield, Eccles \& Rodriguez, 1998).

Weiterhin wurden zwei Skalen aus dem Bereich motivationsbezogener Kognitionen entnommen: die Skalen Selbstwirksamkeitserwartungen und Kontrollerwartungen. Bei beiden Skalen geht es darum, die subjektive Überzeugung des eigenen Einflusses auf schulische Leistungen und Erfolge zu erfassen.

Als volitionale Komponente selbstgesteuerten Lernens wird im Pisa-Fragebogen die Investition von Anstrengung und Ausdauer verstanden. Da diese beiden Handlungskontrollkomponenten schwer trennbar sind, wurden sie in einer Skala mit insgesamt vier Items kombiniert. Gegenstand der Volition sind die kurz vor und die während der eigentlichen Handlungsausführung ablaufenden Kontrollprozesse. Diese willentlichen Prozesse sind zeitlich nach der am Anfang eines Handlungsablaufes angesiedelten Motivationsphase anzusiedeln. 
Um den Bereich der Selbst- und Handlungskontrolle (volitionale Regulation) mittels weiterer Skalen abzudecken, wurde außerdem die Kurzform des Selbststeuerungsinventars (SSI-K-32) von Rheinberg und Wendland (2003) eingesetzt. Kompetenzen der Selbst- und Handlungskontrolle umfassen verschiedene Fähigkeiten der willentlichen Kontrolle, die das Aufrechterhalten eines Handlungsvollzuges ermöglichen. Im SSI-K-32 werden vier Funktionskomponenten der Selbststeuerung mittels 32 Items erfasst. Es handelt sich um die Bereiche: Selbstkontrolle und Zielverfolgung (11 Items), Energiemangel und geringe Konzentration (11 Items), Selbstmotivierung und Emotionskontrolle (7 Items) sowie Procrastination/Aufschieben (6 Items). Die Items werden auf einer fünfstufigen Rating-Skala (,stimmt gar nicht“ bis „stimmt genau“) mit einem Zeitaufwand von insgesamt ungefähr 15 Minuten beantwortet.

\subsubsection{Kognitive Determinanten der Schulleistung}

Die Intelligenz sowie das bereichsspezifische Vorwissen wurden aufgrund ihrer großen Bedeutsamkeit zur Vorhersage von Schulerfolg in das Instrumentarium aufgenommen. Der Konzentrationsfähigkeit wird als wichtige Komponente zur Klärung differentialdiagnostischer Fragestellungen sowie interindividueller Leistungsunterschiede ebenfalls Relevanz zugesprochen.

\section{Intelligenz}

Dem Konstrukt Intelligenz liegt keine einheitliche Definition zugrunde. Stattdessen beruhen Testverfahren zur Messung der Intelligenz auf verschiedenen Intelligenz-Modellen. $\mathrm{Zu}$ diesem Bereich wurde auf die Erfassung einer relevanten Facette - des formallogischen, schlussfolgernden Denkens - fokussiert. Hierfür wurden zwei Untertests aus dem nonverbalen Teil des Kognitiven Fähigkeitstests für 4. bis 12. Klassen (KFT 4-12+R) von Heller \& Perleth (2000) ausgewählt. Bei dem Untertest „Figurenklassifikation“ (N-Test 1) geht es um die korrekte Auswahl einer aus fünf vorgegebenen Figuren zu einem Set gegebener Figuren. Im Untertest „Figurenanalogien“ (N-Test 2) ist ein Paar von Figuren vorgegeben und es soll für eine weitere vorgegebene Figur der dazugehörige Partner analog $\mathrm{zu}$ dem ersten Paar ausgewählt werden. In beiden Tests unterscheiden sich die Figuren hinsichtlich mehrerer Dimensionen (z.B. Größe, Form, Farbe). Beide Tests dauern 20 bzw. 25 Minuten. Die beiden Untertests erfassen anhand von Aufgaben zu figural-räumlichen Fähigkeiten das formallogische, schlussfolgernde Denken, das als eine Kombination von induktivem und de- 
duktivem Denken zu verstehen ist. Diese Komponente der Intelligenz entspricht dem Primärfaktor „Reasoning“ (schlussfolgerndes bzw. formallogisches Denken) in Thurstones Intelligenzmodell, der sieben derartige voneinander unabhängige Primärfaktoren der Intelligenz annahm. Der Faktor „Reasoning“ steht ebenso in engem Zusammenhang mit einer zentralen Komponente des Modells von Cattell, der fluiden Intelligenz. Cattell geht in seinem hierarchischen Modell von einem Generalfaktor der Intelligenz (g-Faktor) aus, der wiederum aus zwei untergeordneten Faktoren besteht - der fluiden und der kristallinen Intelligenz (vgl. Klauer, 2001). Die beiden Konstrukte Reasoning (formallogisches Denken) und fluide Intelligenz stehen in theoretischem Zusammenhang zueinander. Die fluide Intelligenz wird vor allem über Fähigkeiten wie „Induktives Schließen“, „Figurale Beziehungen“ und „Intellektuelle Geschwindigkeit“ gemessen (vgl. Horn, 1968). Sie beschreibt die Fähigkeit, sich neuen Situationen anzupassen und Probleme zu lösen, ohne dass gelerntes Wissen eine Rolle spielt. Das formallogische Denken kommt gerade in solchen Situationen zum Einsatz. In diesem Sinne gelingt es, mittels dieser beiden relativ knappen Untertests zum formallogischen Denken eine grundlegende Komponente intelligenten Verhaltens zu erfassen.

\section{Konzentration}

Zur Erfassung der Konzentrationsfähigkeit wurde der Aufmerksamkeits-Belastungs-Test (d2Test) von Brickenkamp (2002) ausgewählt, da es sich hierbei um einen vielseitig eingesetzten und weitgehend etablierten Test handelt, welcher auf ökonomische Weise im Rahmen einer Gruppentestung ermöglicht, die Konzentrationsfähigkeit objektiv, reliabel und valide zu erfassen. Der d2-Test besteht aus 14 Zeilen mit jeweils 47 Zeichen (d oder p). Jedes dieser Zeichen ist mit 0 bis 3 Strichen versehen, die sich über und unter dem Zeichen befinden. Die Probanden müssen alle d's anstreichen, die mit insgesamt 2 Strichen versehen sind. Für jede Zeile sind 20 Sekunden Zeit vorgesehen, die Personen werden nach 20 Sekunden aufgefordert, bei der nächsten Zeile weiter zu arbeiten. Die gesamte Testdurchführung dauert demzufolge 4 Minuten und 40 Sekunden; mit Instruktion werden ungefähr 10 Minuten benötigt. Der d2-Test kann als Einzel- und Gruppentest eingesetzt werden. Für die eigene Untersuchung wurde dabei als Maß für die Konzentrationsleistung lediglich der sogenannte „Konzentrationsleistungswert“ (KL-Wert) herangezogen. Dieser Wert wird aus der Anzahl der richtig durchgestrichenen relevanten Zeichen ( $d$ mit zwei Stichen) abzüglich der Anzahl an Verwechslungsfehlern gebildet (durchgestrichene d's mit mehr oder weniger als zwei Strichen). Damit ist der KL-Wert laut Brickenkamp (2002) im Gegensatz zu den anderen erfassbaren Leistungsindikatoren verfälschungsresistent, da er durch instruktionswidriges 
Verhalten in Form von wahllosem Durchstreichen aller Zeichen oder oberflächlichem, unkonzentriertem Überspringen von Testteilen nicht in ungerechtfertigter Weise erhöht werden kann.

\section{Bereichsspezifisches Vorwissen}

Um relevante bereichsspezifische Vorkenntnisse zu erfassen, wurden Leistungstests für die Kernfächer Mathematik, Deutsch und Englisch entwickelt ${ }^{2}$. Diese Tests entstanden in Anlehnung an die von der Kultusministerkonferenz formulierten Bildungsstandards (Stand: 10. Klasse). Diese Bildungsstandards legen die Lernziele für die Klassenstufe 10 verbindlich fest, indem sie Kompetenzbereiche definieren und spezielle in der 10. Jahrgangsstufe zu erreichende Zielkompetenzen festlegen. Die Testkonstruktion wurde eng an einigen speziell für diese Schule relevanten Zielkompetenzen orientiert. Aus Gründen der Ökonomie wurde sich pro Test auf eine Bearbeitungszeit von 30 Minuten beschränkt. Die Tests wurden so konzipiert, dass sie ohne Probleme als Gruppenverfahren durchführbar sind (im Test zum Fach Englisch gab es einen Untertest zum Hörverständnis, für welchen jeder Schüler an einem ihm zugewiesenen Platz einen Kopfhörer bekam). Um zu gewährleisten, dass jeder Schüler für sich allein arbeitet, wurden jeweils zwei Versionen A und B entwickelt (im Leistungstest Deutsch wurde für die meisten Untertests lediglich die Reihenfolge der Aufgaben verändert, so dass es sich hierbei um eine Pseudoparallelform handelt). Diese Tests werden im Folgenden ausführlich vorgestellt.

Für das Fach Deutsch wurden Zielkompetenzen aus den Bereichen Lesen, Schreiben und Sprachgebrauch ausgewählt. Zur Erfassung dieser Kompetenzen wurden fachspezifische Testaufgaben mit den Untertests Textverständnis, Wortschatz, Zeichensetzung und Rechtschreibung entwickelt. Die Aufgabe zum Textverständnis besteht darin, zwei in Form von Briefen verfasste Texte, in denen persönliche Meinungen zu einem aktuellen gesellschaftlichen Thema (Graffiti) geäußert werden, zu lesen und dazu fünf Fragen zu beantworten, die sich inhaltlich auf die beiden Texte beziehen. Der Untertest Wortschatz untergliedert sich in zwei Aufgaben zum Finden von Synonymen und Antonymen. Dazu werden mehrere Zeilen mit jeweils sieben zu vergleichenden Wörtern vorgegeben, für welche jeweils zwei vorhandenen Synonyme zu kennzeichnen sind. Zum anderen sind zwei Gruppen mit jeweils zehn Wörtern einander so zuzuordnen, dass für jedes Wort aus der einen Gruppe ein passendes Antonym aus der anderen Gruppe gefunden wird. Um Kenntnisse im Bereich der Zeichensetzung zu überprüfen, wird ein Fließtext dargeboten, in dem die entsprechend

\footnotetext{
${ }^{2}$ Die Leistungstests sind im Anhang nicht aufgeführt, um einen weiteren fairen und objektiven Einsatz im Rahmen zukünftiger Leistungsprognosen zu gewährleisten.
} 
fehlenden Kommata ergänzt werden sollen. Zur Kontrolle der Rechtschreibfähigkeiten wird ein Text mit 12 Rechtschreibfehlern vorgegeben, die $\mathrm{zu}$ markieren und anschließend $\mathrm{zu}$ korrigieren sind.

Für das Kernfach Mathematik wurden relevante Zielkompetenzen ausgewählt und entsprechende fachspezifische Testaufgaben konstruiert, die sich in folgende vier Untertests gliedern: Größenverhältnisse (Ordnen nach Größe), Gleichung (Lösen quadratischer Gleichungen), Zinsrechnung (Lösen einer Aufgabe zur Berechnung von Zinsen), Funktion (Interpretation von Funktionen anhand konkreter Modelle zur Lohnerhöhung). Der Untertest Größenverhältnisse beinhaltet fünf Aufgaben, in denen jeweils zwei Größen vorgegeben werden, deren Verhältnis zueinander durch das Größer-, Kleiner- oder Gleichheitszeichen anzugeben ist. Diese Größen sind dabei in Dezimal-, in Potenzschreibwiese oder als Brüche notiert, so dass entsprechende Grundkenntnisse erforderlich sind, um diese Aufgaben lösen zu können. Beim Untertest Gleichung ist eine Gleichung zu lösen, welche mittels Kenntnis binomischer Formeln und grundlegender Kenntnisse zum Umformen von Gleichungen gelöst werden soll. Um Kenntnisse im Bereich der Zinsrechung zu erfassen, wurde eine Textaufgabe zur Berechnung eines Zinssatzes entwickelt. Beim Untertest Funktion geht es um den Umgang mit der graphischen Darstellung von Daten und ihrer Interpretation. Die Aufgabe ist in verschiedene Elemente unterteilt: zum einen sind zwei Graphen vorgegeben und es sollen aus der Graphik bestimmte Werte abgelesen werden, in einem zweiten Schritt sollen Werte in Prozentwerte umgerechnet werden. Diese Werte sollen dann in einem dritten Schritt in einem Koordinatensystem abgetragen werden. Schließlich soll eine Frage zum Verständnis zweier Graphiken beantwortet werden.

Für das Kernfach Englisch sollten Zielkompetenzen aus den Bereichen Hörverständnis, Leseverständnis, sprachlicher und schriftlicher Ausdruck erfasst werden. Dementsprechend wurden fachspezifische Aufgaben konstruiert, die in folgende drei Untertests untergliedert sind: Matching (Zuordnen), Einsetzen und Übersetzung. Bei dem Untertest Matching sind Fertigkeiten des Textverstehens gefragt. Hier geht es darum, Informationen aus verschiedenen, schriftlich vorgegebenen kurzen englischen Texten zum Beantworten einer inhaltsbezogenen Aufgabe zu nutzen. Es sollen je nach der Testversion entweder Buchklappentexte zur Beschreibung des Inhaltes eines Buches einem bestimmten Leserwünschen zugeordnet werden oder Fragen auf der Grundlage kurzer Ratschläge für das Autofahren im Winter beantwortet werden. Kompetenzen aus dem Bereich des Hörverstehens werden mittels des Untertests Einsetzen erfasst. Dabei wird den Schülern per Köpfhörer ein Text vom Tonband vorgespielt. Je nach Testversion handelt es sich dabei entweder um eine 
Radiomitteilung bzw. ein Gespräch in einer Kantine zwischen zwei fiktiven Personen. Vorgegebene Fragen sind mit ,ja“ oder „,nein“ zu beantworten. Der Untertest Übersetzung dient der Erfassung von kommunikativen Kompetenzen. In der fiktiven Rolle eines Übersetzers sollen englische Sätze ins Deutsche und deutsche Sätze ins Englische überführt werden.

\subsubsection{Motivationale Determinanten der Schulleistung}

Zur Erweiterung des Instrumentariums um nicht kognitive Determinanten wurden verschiedene Komponenten aus dem motivationalen Bereich ausgewählt. Zunächst werden Verfahren $\mathrm{zu}$ den Bereichen Lern- und Leistungsmotivation vorgestellt. Des Weiteren werden Instrumente aus dem Bereich der motivationsbezogenen Kognitionen, zum schulischen Selbstkonzept und schulischen Selbstwirksamkeitserwartungen, erläutert.

\section{Leistungsmotivation}

Zur Erfassung der Leistungsmotivation wurden das LeistungsmotivationsinventarKurzversion (LMI-K) von Schuler \& Prochaska (2001) und die zwei Skalen Lernziele und Arbeitsvermeidung aus den Skalen zur Erfassung der Lern- und Leistungsmotivation (SELLMO) von Spinath, Stiensmeier-Pelster, Schöne und Dickhäuser (2002) ausgewählt.

Das Leistungsmotivationsinventar (LMI) von Schuler und Prochaska (2001) versteht Leistungsmotivation als globale Verhaltensorientierung und ermöglicht anhand von 17 Skalen eine differenzierte Beschreibung des Konstruktes. Die Autoren haben versucht, in ihrem Instrument verschiedene Arten von Verhaltenstendenzen, die mehr oder weniger in direktem Zusammenhang zur Leistungsmotivation stehen, zu integrieren. Zum einen handelt es sich um häufig mit Leistungsmotivation assoziierte Dimensionen, zum anderen um Verhaltenstendenzen, die in der Regel weniger häufig in einen Zusammenhang mit Leistungsmotivation gebracht werden. Des Weiteren wurden auch Dimensionen integriert, welche theoretisch in keinem direkten Bezug zu Leistungsmotivation stehen, für welche man sich allerdings theoretisch ein Zusammenwirken mit Leistungsmotivation vorstellen kann (z.B. die Attributionsneigung). Nicht zuletzt wurden Persönlichkeitsmerkmale berücksichtigt, für welche ein Zusammenhang mit den Kernaspekten der Leistungsmotivation vorstellbar ist, wie beispielsweise das Pflichtbewusstsein aus der Gewissenhaftigkeit, die Dominanz aus der Extraversion (vgl. Schuler \& Prochaska, 2001). Die Autoren sehen Leistungsmotivation weniger als abgegrenztes Konstrukt, als als globale Verhaltensorientierung, an der vielfältige 
Aspekte der Persönlichkeit beteiligt sind. Der Fragebogen ist sowohl in der Berufseignungsdiagnostik als auch in der Forschung einsetzbar. Die Gesamtform enthält 10 Items pro Skala (insgesamt 170 Items).

Die für die vorliegende Untersuchung ausgewählte Kurzform des LMI (LMI-K) beinhaltet 30 Items, die den Gesamtwert der Normalversion besonders gut repräsentieren. Es wurden also Items ausgewählt, welche die höchsten Item-Gesamttest-Korrelationen aufweisen. Auch der Kurzversion wurde das Verständnis von Leistungsmotivation als ein sehr breites Konstrukt im Sinne der Ausrichtung weiter Anteile der Persönlichkeit auf die Leistungsthematik zugrunde gelegt. Aus diesem Grunde wurde darauf geachtet, dass in der Kurzform möglichst viele Einzeldimensionen vertreten sind. Diese können aus Reliabilitätsgründen nicht gesondert ausgewiesen bzw. ausgewertet werden. Die Items werden auf einer 7-stufigen Skala beantwortet („,1“: „trifft gar nicht $\mathrm{zu}^{“}$, , ,7“: „,rifft vollständig zu“). Die Durchführung beschränkt sich auf 10 Minuten.

Das Leistungsmotivationsinventar wurde hauptsächlich aufgrund der Breite seines theoretischen Ansatzes ausgewählt. Die Kurzversion überzeugte aufgrund ihrer Kürze und der guten Repräsentativität der Langform. Des Weiteren ist der LMI gerade für Fragen der Personalauswahl geeignet und ausgewiesen und enthält unter anderem auch Normen für Wirtschaftsgymnasiasten (Schuler \& Prochaska, 2001).

Zwei weitere Skalen ergänzen den LMI-K insofern sinnvoll, dass für die vorliegende Fragestellung besonders wichtige Bereiche von Leistungsmotivation noch einmal auf andere Art und Weise erfragt werden. Es handelt sich um die Skalen Lernziele und Arbeitsvermeidung aus dem „SELLMO“, den Skalen zur Erfassung der Lern- und Leistungsmotivation (vgl. Spinath et al., 2002). Dem SELLMO liegt der Gedanke der Zielorientierung menschlichen Verhaltens zugrunde. Er erfasst insgesamt vier Arten von Zielen: Lernziele, Annäherungs-Leistungsziele, Vermeidungs-Leistungsziele und die Tendenz zur Arbeitsvermeidung. Die Dimension Lernziele umschreibt das Bestreben von Personen, bei der Beschäftigung mit Aufgaben eigene Kompetenzen zu erweitern. In der Dimension der Annäherungs-Leistungsziele drückt sich das Bestreben aus, eigenes Wissen und Können zu zeigen, während Vermeidungs-Leistungsziele dann im Vordergrund stehen, wenn jemand als unzureichend wahrgenommene eigene Kompetenzen zu verbergen sucht. Die Dimension der Arbeitsvermeidung beschreibt, inwieweit jemand bemüht ist, möglichst wenig Arbeit in die Erfüllung von Aufgaben zu investieren.

Aus Gründen der Ökomomie wurden nur die beiden für die eigene Untersuchung relevantesten Skalen Lernbereitschaft und Arbeitsvermeidung ausgewählt. Personen mit 
hohen Lernzielen streben nach Erweiterung der eigenen Kompetenzen, sehen gestellte Aufgaben als Herausforderung und Gelegenheit zum Lernen. Zahlreiche Studien belegen, dass hohe Lernziele langfristiges Engagement und gute Leistungen sichern (Spinath et al., 2002). Lernziele werden eher mit intrinsischem Lernen assoziiert und hängen auch stärker mit diesem zusammen als Leistungsziele. In experimentellen Studien konnte bestätigt werden, dass eine Orientierung auf Lernziele im Vergleich zu einer Orientierung auf Leistungsziele durchschnittlich zu besseren Leistungsergebnissen führt (vgl. Utman, 1997). Die Motivation bei Zielen der Arbeitsvermeidung liegt in dem Bemühen, möglichst wenig Arbeit zu investieren. Naturgemäß geht diese Tendenz kurz- und langfristig mit geringem Engagement und schlechten Leistungen, wie auch mit geringem Interesse und geringer intrinsischer Motivation einher. Es ist empirisch belegt, dass hohe Arbeitsvermeidungstendenzen mit schlechteren Noten zusammenhängen. Im Rahmen der geplanten Untersuchung wird davon ausgegangen, dass geeignete Schüler auf dieser Skala niedrige Werte erzielen.

Beide Skalen stellen gegensätzliche Pole eines für die vorliegende Fragestellung relevanten individuellen Merkmales dar und ermöglichen eine sinnvolle Ergänzung des LMIK zur Erfassung der Leistungsmotivation.

\section{Motivationsbezogene Kognitionen}

Es wurde hierzu das von Rost und Sparfeldt (2002) entwickelte Verfahren zur Messung des differentiellen Selbstkonzepts schulischer Leistungen und Fähigkeiten (DISK-Gitter) ausgewählt. Das DISK-Gitter entstand in Orientierung an der Skala zur Erfassung des Selbstkonzepts schulischer Leistungen und Fähigkeiten (SKSLF, Rost \& Lamsfuß, 1992), die wiederum in Anlehnung an die amerikanische „Self-Concept as a Learner Scale“ von Waetjen (1963, zitiert nach Burns, 1979) und an die SIQ-Subskala „Leistungsselbstkonzept“ (SeiffgeKrenke, 1987) entwickelt wurde. Aus Ökonomiegründen wurde die aus 10 Items bestehende Originalskala um zwei Items gekürzt und in der Formulierung teilweise dem altersentsprechenden Sprachgebrauch angeglichen. In dieser fachbezogenen Form des DISKGitters wurde an geeigneter Stelle des Items das jeweilige Fach eingefügt (in der Originalversion handelte es sich um die Fächer Mathematik, Deutsch, Physik und Geschichte). Das aus acht Items bestehende DISK-Gitter wird auf einem sechsstufigen Antwortformat $\left(1=\right.$,trifft gar nicht $\mathrm{zu}^{\text {“ }}$ bis $6=$,trifft genau $\mathrm{zu}^{\text {“ })}$ ) bearbeitet. Empirische Studien belegen, dass es durchaus sinnvoll ist, das Selbstkonzept möglichst spezifisch zu erfassen, also auf Ebene der einzelnen Fächer (vgl. Moschner \& Dickhäuser, 2006). Ein großer Vorteil des Instrumentes ist zusätzlich die hohe Ökonomie, die zum einen durch die 
Kürze des DISK-Gitters sowie zum anderen durch die sehr ökonomische Anordnung der Items erreicht wird (es sind nur acht Items zu lesen) und somit das Ausfüllen auf wenige Minuten beschränkt. Dies ist ein entscheidender Vorteil gegenüber anderen, ebenfalls gut etablierten Verfahren, wie beispielsweise das von Dickhäuser et al. (2002) entworfene Instrument zur Erfassung des akademischen Selbstkonzeptes, welches zusätzlich zwischen verschiedenen Bezugsnormen trennt und dadurch deutlich länger ist. Ein weiterer Vorteil des Instrumentes ist die einfache Erweiterung um andere Fächer, die methodisch problemlos möglich ist (Rost \& Sparfeldt, 2002). Im entwickelten Diagnoseinstrumentarium wurde das DISK-Gitter für die Fächer Mathematik, Deutsch und Englisch angepasst.

Für die Erfassung der schulischen Selbstwirksamkeitserwartung wurde die Skala zur Schulbezogenen Selbstwirksamkeitserwartung (WIRKSCHUL) von Jerusalem \& Satow (1999) eingesetzt, welche die Kompetenzerwartungen von Schülern im Umgang mit schulischen Anforderungen anhand von 7 Items auf einer vierstufigen Ratingskala erfasst (von „Trifft

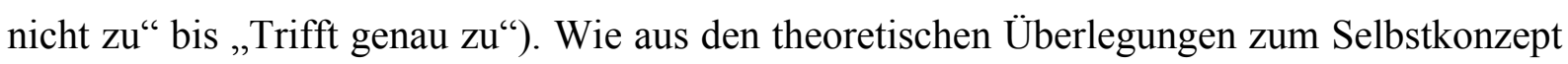
hervorgeht, werden Selbstwirksamkeitserwartungen als wichtiger Bestandteil des Selbstkonzeptes angesehen und sind umso stabiler je spezifischer nach ihnen gefragt wird. Da Selbstwirksamkeitserwartungen äußerst wichtig für gelungene Lernprozesse und gute Leistungen sind, wurde diese Skala in das Instrument mit aufgenommen. Die Bearbeitungszeit beträgt ungefähr 5-10 Minuten und die Skala ist in einem Altersbereich bis zu einschließlich 16 Jahren einsetzbar.

Um die Bedeutsamkeit der in das Diagnoseinstrumentariums eingehenden Verfahren und Tests bestimmen zu können, bedarf es einer Evaluation und Validierung dieser Komponenten. In einem ersten Schritt im Rahmen der ersten Studie wurde das vorläufige Instrumentarium hinsichtlich seiner generellen Eignung überprüft sowie anhand verschiedener relevanter Kriterien zum Schulerfolg optimiert und auf seine wichtigsten Bestandteile gekürzt. Schließlich wurde das Diagnoseinstrument in der zweiten Studie an einer anderen Stichprobe eingesetzt und anhand dieser Daten weiter validiert. Der zeitliche Rahmen des Gesamtprojektes erstreckte sich von August 2004 bis Juli 2006 (siehe Abbildung 4). 


\section{3 Überblick zur Studie 1: Entwicklung des Diagnoseinstrumentariums}

Im Rahmen der ersten Studie sollte das Instrumentarium hinsichtlich der Eignung der enthaltenen Komponenten überprüft und darauf aufbauend in einem ersten Optimierungsschritt auf seine relevantesten Bausteine reduziert werden. Die Verfahren und Tests des Diagnoseinstrumentariums aus den Bereichen Vorwissen, kognitive Grundfertigkeiten, Motivation und Selbstregulation wurden erstmalig im August 2004 an einem kurz zuvor eingeschulten 11. Jahrgang erhoben. Zur Prüfung der Prädiktionskraft der im Instrumentarium enthaltenen Verfahren (sogenannte Prädiktorvariablen oder auch Prädiktoren) wurden nach 45 Monaten an der gleichen Stichprobe relevante Maße des Schulerfolges (sogenannte Validitätskriterien) erfasst. Mittels des im Kapitel 2 vorgestellten Verfahrens der multiplen Regression wurde dann ermittelt, welche der im August 2004 erhobenen Prädiktorvariablen zur Aufklärung der später erhobenen Validitätskriterien beitrugen. Des Weiteren konnten multiple Regressionsanalysen zur Vorhersage der Validitätskriterien aufgestellt werden. Für eine erste Optimierung des Instrumentariums wurden die Prädiktoren aus dem vorläufigen Instrumentarium entnommen, welche keinen Beitrag zur Varianzaufklärung leisteten. Das um die irrelevanten Prädiktorvariablen gekürzte Diagnoseinstrumentarium wurde im Rahmen der zweiten Studie an einer weiteren Stichprobe eingesetzt.

\section{4 Überblick zur Studie 2 (Hauptstudie): Validierung des Diagnoseinstrumentariums}

In der zweiten Studie wurde das gekürzte Instrumentarium an einer zweiten Kohorte eingesetzt. Es handelte sich um Schüler, die sich für die Aufnahme in die 11. Jahrgangsstufe des Wirtschaftsgymnasiums beworben hatten. Das Diagnoseinstrumentarium wurde parallel zum ehemaligen Vorgehen der Schule bei der Auswahl der Schüler für die 11. Jahrgangsstufe des Wirtschaftsgymnasiums durchgeführt. Mithilfe der an der ersten Stichprobe erstellten multiplen Regressionsgleichungen zur Vorhersage der Validitätskriterien wurde eine Rangreihe über alle Bewerber erstellt, die richtungsweisend für die Auswahl mit dem neuen Instrumentarium war. Da beide Auswahlverfahren berücksichtigt wurden, konnten die ausgewählten Schüler in drei Gruppen eingeteilt werden: a) nach dem ehemaligen Verfahren der Schule ausgewählt, b) nach dem neu entwickelten Diagnoseinstrumentarium ausgewählt 
und c) nach beiden Vorgehensweisen ausgewählt. Bei diesen Schülern wurden nach 9-16 Monaten ebenfalls Validitätskriterien erhoben. Anhand verschiedener Analysen konnte das Diagnoseinstrumentarium hinsichtlich seiner prognostischen Validität weiter evaluiert und gekürzt sowie hinsichtlich seiner Güte der Schülerauswahl mit dem ehemaligen Verfahren verglichen werden.

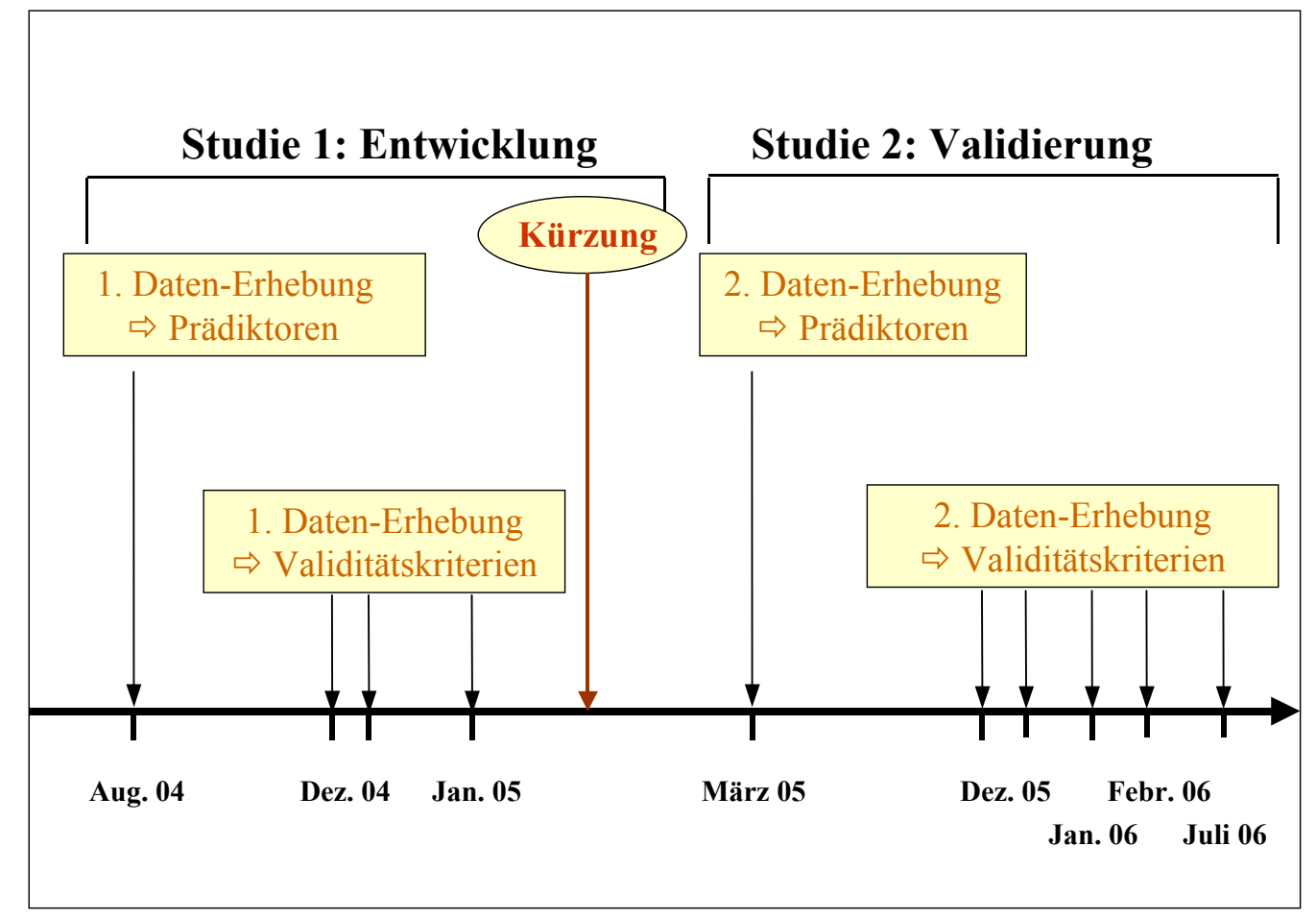

Abbildung 4: Zeitlicher Ablauf des Gesamtprojektes bestehend aus 2 Teilstudien. 


\section{Studie 1: Entwicklung des Diagnoseinstru- mentariums}

In dieser ersten Studie wurde das erstellte Diagnoseinstrumentarium mit den enthaltenen Prädiktorvariablen zur Erfassung des Vorwissens sowie relevanter „psychologischer“ Merkmale $^{3}$ einer empirischen Prüfung unterzogen. Eine Übersicht über diese Komponenten bietet Tabelle 3 .

Tabelle 3: Übersicht über die im Diagnoseinstrumentarium enthaltenen Prädiktorvariablen

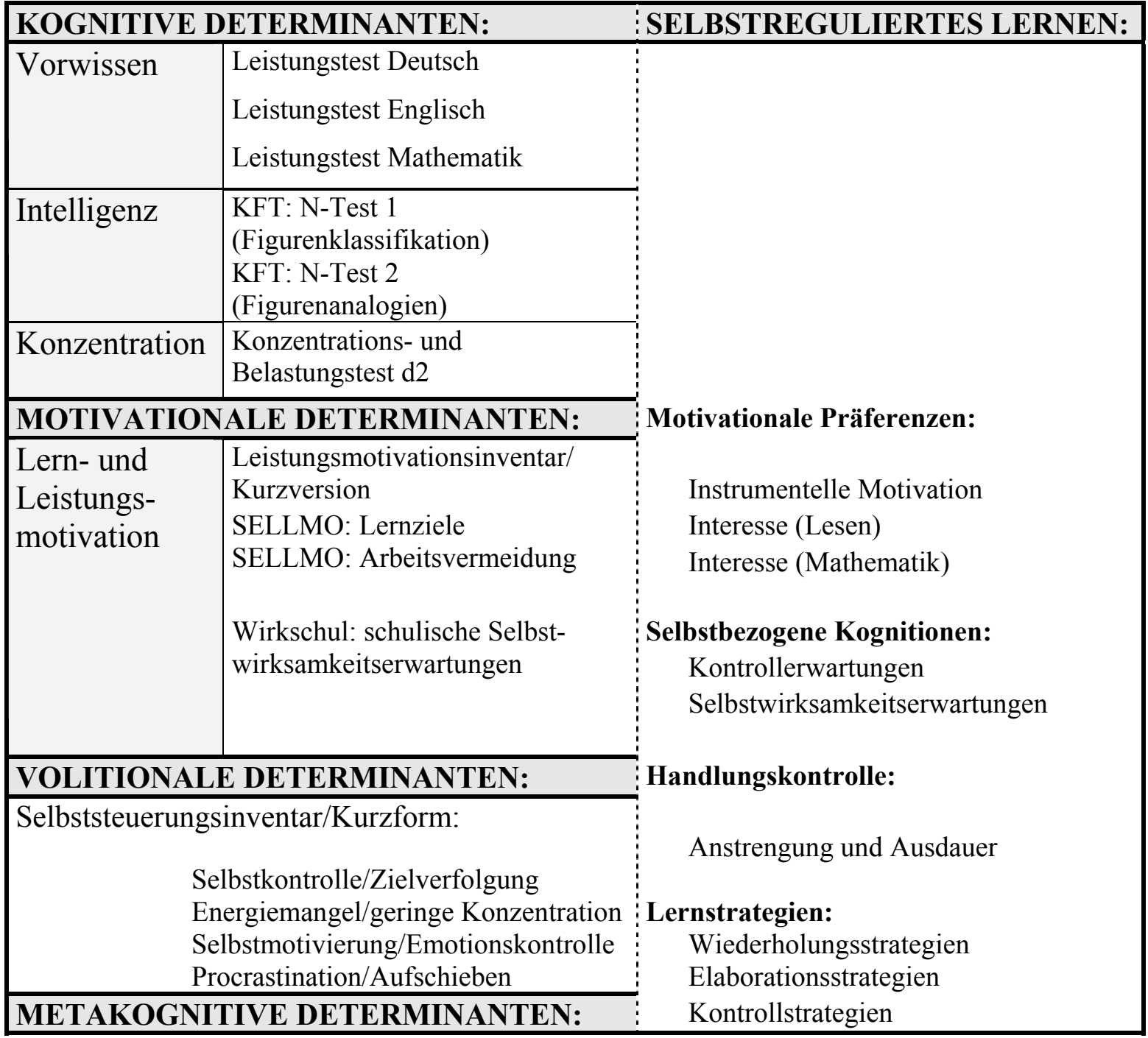

Anmerkung. Für alle aufgeführten Skalen wird ein positiver Zusammenhang mit der Schulleistung erwartet. Die Skalen Arbeitsvermeidung sowie Procrastination/Aufschieben stehen in negativem Zusammenhang zur Schulleistung und wurden dementsprechend umgepolt.

\footnotetext{
${ }^{3}$ Die Konstrukte kognitive Grundfertigkeiten (schlussfolgerndes Denken), Motivation und Selbstregulation bzw. die Operationalisierungen dieser Konstrukte werden im Weiteren vereinfachend als ,psychologische“ Merkmale bzw. ,psychologische“ Verfahren bezeichnet. Die hierfür gewählte eher umgangssprachliche Bezeichnung soll die Lesbarkeit und Verständlichkeit erleichtern.
} 


\subsection{Fragestellungen}

Das herkömmliche Auswahlverfahren beruhte auf der Annahme, dass frühere Leistungsbewertungen in Form von Schulnoten die besten Prädiktoren für spätere Schulleistungen sind. Grundlage für die Auswahl waren die Abschlusszeugnisnoten der Bewerber in den Fächern Deutsch, Mathematik und Englisch, welche nach einem auf Erfahrungswerten der Schule beruhenden Schlüssel gewichtet wurden. Diese Gewichtung sollte dem unterschiedlichen Anforderungsniveau der abgebenden Schulen (Gymnasium, Real- und Hauptschule) Rechnung tragen und somit eine bessere Vergleichbarkeit der Noten gewährleisten. Die Konzeption des neuen Systems wurde von der Annahme geleitet, dass fachspezifische Vorkenntnisse und fächerübergreifende Kompetenzen einen höheren prädiktiven Wert für zukünftige schulische Leistungen an dieser Schule besitzen als die Schulnoten der Herkunftsschulen. Sofern die Güte der beiden Verfahren allein nach ökonomischen Gesichtspunkten beurteilt würde, wäre das ehemalige Verfahren aufgrund des geringeren Zeitaufwandes überlegen. Es interessiert allerdings stärker die Frage danach, welches der beiden Verfahren eine höhere prognostische Validität aufweist beziehungsweise wie hoch der prognostische Zugewinn ist, der einen Einsatz des neuen aufwendigeren Verfahrens rechtfertigen würde. Somit kann folgende Hypothese formuliert werden:

\section{Hypothese zur erhöhten Prognosesicherheit des Diagnoseinstrumentariums:}

Das Diagnoseinstrumentarium zur Auswahl von Schülerinnen und Schülern für das Wirtschaftsgymnasium auf der Basis von an Bildungsstandards orientierten Leistungstests und „psychologischen“ Verfahren der Bereiche kognitive Grundfertigkeiten, Motivation und Selbstregulation erlaubt eine bessere Vorhersage der schulischen Eignung als die bisherigen Schulnoten.

Um ein effizientes und ökomomisches Verfahren zu entwickeln ist es wichtig, aussagekräftige Prädiktoren zu identifizieren und Komponenten aus dem Instrumentarium zu entfernen, die überflüssige oder redundante Informationen bereitstellen. Es stellt sich die Frage, welche der in die erste Version des Diagnoseinstrumentariums aufgenommenen Verfahren und Tests zur Erklärung der Schulleistung beitragen.

Mit einem Fokus auf die Bereiche Kognition, Motivation, Volition und Metakognition, zu denen Verfahren ausgewählt wurden, kristallisiert sich eine konkretere Fragestellung heraus. Der wichtige Beitrag des Vorwissens sowie kognitiver 
Grundfertigkeiten zur Vorhersage von Schulerfolg ist vielfach empirisch belegt. Hinsichtlich des Einflusses motivationaler, volitionaler und metakognitiver Komponenten des Lernens ist die Befundlage weniger eindeutig. In letzter Zeit wird allerdings auch diesen Variablen eine Bedeutung zur Erklärung von interindividuellen Unterschieden in der Schulleistung zugesprochen. Es stellt sich hier folglich die Frage, inwieweit die ausgewählten Prädiktoren der Bereiche kognitive Grundfertigkeiten, Motivation und Selbstregulation zu einer Verbesserung der bereits durch die vorwissensrelevanten Leistungstests erreichten Prädiktionskraft beitragen.

Diese Ausführungen weisen darauf hin, dass hier weniger hypothesenprüfend, als vielmehr explorativ vorgegangen wird. Aus diesem Grunde werden hierzu Fragestellungen anstelle von Hypothesen formuliert:

\section{Fragestellungen zu bedeutsamen Prädiktorvariablen und der inkrementellen Validität „psychologischer" Merkmale:}

Welche Leistungen und „psychologischen“ Merkmale der Bereiche kognitive Grundfertigkeiten, Motivation und Selbstregulation tragen zur Aufklärung des Schulerfolges bei?

Wird durch den Einbezug der psychologischen Merkmale noch eine Verbesserung der Prognosekraft im Vergleich zur allein durch die Leistungstests erreichten Vorhersage erzielt?

\subsection{Methode}

\subsubsection{Ablauf der Untersuchung}

Die Datenerhebung dieser ersten Studie beinhaltete zwei globale Messzeitpunkte: die Erhebung der im Diagnoseinstrumentarium enthaltenen Prädiktorvariablen im August 2004 sowie die Erhebung der Validitätskriterien im Dezember 2004/ Januar 2005.

Zum ersten Messzeitpunkt im August 2004 wurden die Schüler parallel in vier Gruppen mit dem erstellten Diagnoseinstrumentarium untersucht. Die Durchführung kann strukturell in zwei Einheiten unterteilt werden: 1.) die Durchführung der Leistungstests der Fächer Mathematik, Deutsch und Englisch sowie 2.) der Einsatz psychologischer Fragebögen und Tests. Der erste Teil wurde durch die jeweiligen Fachlehrer betreut. Instruktionen zur Durchführung der Leistungstests waren auf den Testbögen vermerkt. Es wurden hierfür 
jeweils 30 Minuten Bearbeitungszeit eingeplant; zwischen den einzelnen Tests waren kurze Pausen vorgesehen. Anschließend wurden die Fragebögen und Tests $\mathrm{zu}$ den individuellen psychischen Merkmalen durchgeführt. Die Testleiter waren zuvor ausführlich über die Durchführung instruiert worden und orientierten sich an den notwendigen Unterlagen und standardisierten Instruktionen für die Durchführung (die Instruktion zu den Fragebögen ist im Anhang D zu finden). Es wurden zunächst die Untertests des Kognitiven Fähigkeitstests (NTest 1, N-Test 2), dann der Aufmerksamkeits-Belastungstest (Test d2) und drei Fragebögen, die die ausgewählten Skalen der Bereiche Motivation und Selbstregulation enthielten, durchgeführt (die drei Fragebögen mit den relevanten Skalen sind im Anhang F-H einzusehen). Die Schüler wurden vor jedem Test (N-Test 1, N-Test 2, d2-Test) entsprechend der aus den Handbüchern vorliegenden Angaben instruiert.

Zum zweiten Messzeitpunkt nach 4-5 Monaten wurden Validitätskriterien des Schulerfolges der Schüler erhoben. Im Zeitraum von Dezember 2004 bis Januar 2005 wurden drei verschiedene Maße zum Schulerfolg erfasst. Im Dezember wurde von 4 Fachlehrern ein Eignungsurteil für jeden Schüler eingeholt. Auf einer vierstufigen Ratingskala beurteilten diese Lehrer (für die Fächer Deutsch, Mathematik, Englisch und $\mathrm{BRC}^{4}$ ) getrennt voneinander jeden Schüler hinsichtlich seiner Eignung für Oberstufe des Wirtschaftsgymnasiums. Es handelt sich hierbei um eine selbst konstruierte Skala bestehend aus vier Items. Der kurze Fragebogen wurde an alle Fachlehrer verteilt und von diesen ausgefüllt. Des Weiteren wurde im Dezember 2004 die subjektive Leistungszufriedenheit der Schüler erhoben. Es handelt sich hierbei um einen kurzen, ebenfalls selbst konstruierten, Fragebogen von 10 Items. Dieser wurde an einem Vormittag hintereinander in den vier Klassen durchgeführt. Die Schüler wurden auch hier ausführlich zum Ausfüllen des Bogens instruiert. Die Durchführung dieser Befragung dauerte ungefähr 20 Minuten. Im Januar 2005 wurden die Halbjahresnoten der Schüler als weiteres Kriterium für Schulerfolg eingeholt und mit in die Analysen einbezogen. Die in diesem Zeitraum erhobenen Kriterien gingen gemeinsam mit den zuvor erhobenen Prädiktoren in die multiplen Regressionsanalysen zur Evaluierung und Optimierung des Instrumentariums ein.

\footnotetext{
${ }^{4}$ BRC bezeichnet ein Wirtschaftsfach, welches die Bereiche Betriebswirtschaft, Rechnungswesen und Controlling beinhaltet.
} 


\subsubsection{Variablen}

Die für das Diagnoseinstrumentarium ausgewählten Leistungstests, „psychologischen“ Testverfahren sowie Fragebögen stellen die unabhängigen Variablen, die im zeitlichen Ablauf später erhobenen Variablen die abhängigen Variablen dar. Unabhängige Variablen sind im Kontext der multiplen Regression als Prädiktoren, die abhängigen Variablen als Kriterien (im Weiteren auch als Validitätskriterien bezeichnet) zu verstehen, wobei es darum geht, ein Kriterium durch einen beziehungsweise mehrere Prädiktoren zu erklären.

\section{Unabhängige Variablen (Prädiktoren)}

Als Prädiktoren wurden die von den Schülern in den Tests und Verfahren des Diagnoseinstrumentariums erzielten Werte eingesetzt (siehe hierzu Tabelle 19 im Anhang R). Es handelt sich hierbei um die Prozentwerte der erzielten Ergebnisse in den Leistungstests, die erreichte Punktsumme der zwei Untertests des Kognitiven Fähigkeitstests, den Konzentrationsleistungswert des Aufmerksamkeits- und Belastungstests sowie um Mittelwerte zu den in den Fragebögen enthaltenen Skalen der Bereiche Motivation und Selbstreguliertes Lernen. Diese konkreten Werte bilden die Prädiktorwerte, die in die multiplen Regressionsanalysen zur Vorhersage der Validitätskriterien eingingen. Die Prädiktorvariablen wurden bereits ausführlich unter Kapitel 3 dargestellt. Da es sich bei den ausgewählten Skalen und Tests zur Messung individueller psychischer Merkmale um bereits erprobte und weitgehend optimierte Verfahren handelt, ist gewährleistet, dass diese Verfahren hinsichtlich der Gütekriterien Objektivität, Validität und Reliabilität erprobt sind und befriedigende bis gute Kennwerte aufweisen (siehe Tabelle 17 in Anhang A).

\section{Abhängige Variablen (Validitätskriterien)}

Zur Optimierung des Diagnoseinstrumentariums wurden einige Zeit nach der Erhebung der unabhängigen Variablen abhängige Variablen (Kriterien bzw. Validitätskriterien) erfasst. Es handelt sich hierbei um Variablen, die den Schulerfolg der Schüler möglichst gut abbilden sollen. Hierzu gehören ein Urteil der Schüler-Eignung durch mehrere Fachlehrer, eine Einschätzung der eigenen Leistungszufriedenheit durch die Schüler sowie die Halbjahresnoten. Diese Validitätskriterien werden im Folgenden konkreter dargestellt.

Im Dezember 2004 wurden für jeden Schüler vier Lehrerurteile zur Eignung eingeholt. Die Fachlehrer der Fächer Mathematik, Deutsch, Englisch und BRC (Betriebswirtschaft, 
Rechnungswesen und Controlling) beurteilten die Schüler anhand von vier Items auf einer vierstufigen Ratingskala (von sehr (geeignet) bis gar nicht (geeignet)). Die Items bestanden aus kurzen und klaren Fragen, wie zum Beispiel: „Für wie geeignet halten Sie oben genannten Schüler/Schülerin für Ihre Schule?“ oder „Wie überzeugt sind Sie, dass oben genannter Schüler/Schülerin den Abschluss an Ihrer Schule schaffen wird?“ (die Lehrer gaben ihre Antworten direkt in eine Excel-Datei ein und bezogen sich in ihren Antworten jeweils auf einen bestimmten Schüler). Mittels der selbst erstellten Skala sollte die Eignung für die Oberstufe bezogen auf das Fachgymnasium Wirtschaft sowie die Fähigkeit, das Abitur erfolgreich zu absolvieren, eingeschätzt werden (Fragebogen siehe Anhang B). Insgesamt lagen für jeden Schüler vier verschiedene Eignungsurteile von Fachlehrern vor. Diese Angaben wurden zunächst für jedes Fach/jeden Lehrer über die vier Skalenitems zusammengefasst und schließlich wurde ein Mittelwert über alle Lehrerurteile für jeden Schüler gebildet. Dieser Wert ging als Kriteriumswert in die Regressionsanalysen ein.

Ebenfalls im Dezember 2004 wurde eine Befragung der Schüler hinsichtlich ihrer eigenen Leistungszufriedenheit durchgeführt. Dieses Kriterium sollte als Gegenstück zur Beurteilung durch die Lehrer auch die Schülerseite berücksichtigen. Da die Frage nach den eigenen Leistungen vermutlich relativ leicht verfälschbar ist, wurde nicht direkt nach der Einschätzung des eigenen Schulerfolges, sondern nach der Zufriedenheit bezüglich der eigenen Leistungen gefragt. Es handelt sich hierbei um einen kurzen selbst entwickelten Fragebogen mit zehn Aussagen, die auf einer vierstufigen Ratingskala (von „stimmt genau“ bis ,stimmt gar nicht*) beantwortet werden (Itembeispiele: „Nach den ersten Monaten an der Arnoldi-Schule bin ich zuversichtlich, dass ich das Abitur schaffe.“, „Wenn ich sehe, was wir an der Arnoldi-Schule können müssen, halte ich mich für sehr geeignet.“, Fragebogen siehe Anhang C). Als Wert für die subjektive Schülerzufriedenheit wurde ein Mittelwert über den Fragebogen gebildet, welcher als Kriterium in die Analysen einging.

Im Januar 2005 wurden die Halbjahresnoten der Schüler als weitere relevante Kriterien zur Operationalisierung des Schulerfolges eingeholt. Es wurden die Noten der Kernfächer Mathematik, Deutsch, Englisch und BRC (Betriebswirtschaft, Rechnungswesen und Controlling) sowie eine Durchschnittsnote über alle Halbjahresnoten ${ }^{5}$ als Operationalisierungen schulischer Leistungen herangezogen.

Insgesamt standen mit dem Eignungsurteil der Lehrer, der Einschätzung der eigenen Leistungszufriedenheit sowie den Halbjahresnoten 7 Validitätskriterien zur Verfügung, um im

\footnotetext{
${ }^{5}$ Die durchschnittliche Halbjahresnote beinhaltet die Fächer BRC, Volkswirtschaft, Informatik, Deutsch, Englisch, Mathematik, Naturwissenschaft (wahlweise Biologie, Physik, Chemie), Sprache (wahlweise Französisch oder Spanisch), Religion oder Werte und Normen sowie Politik und Fachpraxis.
} 
Weiteren zu analysieren, inwieweit individuelle Unterschiede in den Prädiktormaßen Unterschiede der Kriteriumsmaße erklären beziehungsweise vorhersagen können.

\subsubsection{Analysestrategie zur Ermittlung aufgeklärter Kriteriumsvarianz}

Für die methodische Auswertung wurde das Verfahren der multiplen linearen Regression eingesetzt. Im Rahmen der Regressionsanalyse wird der Zusammenhang zwischen einer abhängigen und einer oder mehreren unabhängigen Variablen betrachtet. Mit Hilfe der Regressionsanalyse können dann die unterstellten Beziehungen überprüft und quantitativ abgeschätzt werden. Dieses Verfahren eignet sich sowohl für die Erklärung von Zusammenhängen als auch für die Durchführung von Prognosen (Backhaus, Erichson, Plinke \& Weiber, 2000). Bei der Vorhersage von Zusammenhängen sollen aus einer Gruppe potentiell wichtiger Merkmale solche selegiert werden, durch die das Kriterium am besten vorhergesagt werden kann. Die Regressionsanalyse unterstellt eine eindeutige Richtung des Zusammenhangs unter den Variablen, die nicht umkehrbar ist. Es wird also theoretisch ein kausaler Wirkzusammenhang angenommen (vgl. Backhaus et al., 2000).

Ein Vorteil der dargestellten multiplen Regressionsanalyse besteht zum einen darin, dass mehrere Prädiktoren zur Vorhersage eines Kriteriums berücksichtigt werden können. Zum anderen lassen sich über die multiple Regressionsanalyse die Gewichte der einzelnen Variablen am Zustandekommen der Kriteriumsleistung feststellen, so dass man die Bedeutung der einzelnen Variablen im Hinblick auf die Aufklärung der Kriteriumsvarianz abschätzen kann (Sauer \& Gamsjäger, 1996).

Die Methode der Regressionsanalyse baut auf verschiedenen wichtigen Annahmen auf. Zum einen wird davon ausgegangen, dass die unabhängigen und abhängigen Variablen metrisch skaliert sind. Eine weitere Voraussetzung ist die theoretisch begründbare Annahme einer eindeutigen Kausalrichtung des Zusammenhangs. Als günstige Ausgangsbedingung gilt die ausreichende Qualität der Prädiktor- und Kriteriumsvariablen.

Obwohl die Technik der Regressionsanalyse im pädagogisch-psychologischen Bereich äußerst verbreitet ist und flexibel eingesetzt wird, gibt es auch einige Besonderheiten dieses Verfahrens, die beachtet werden sollten. So können die spezifischen Anteile von Prädiktoren an der Kriteriumsvarianz nur dann exakt bestimmt werden, wenn die Prädiktoren unkorreliert, also voneinander unabhängig, sind. Da diese Forderung selten vollständig erfüllt ist, ergibt sich hier ein Grund für Verzerrungen der Regressions-Schätzparameter. Die Vorhersagegüte hängt des Weiteren mit der Reliabilität der Prädiktoren zusammen. Je geringer die Reliabilität 
der Prädiktoren ausfällt, desto größer ist die Verzerrung in den Schätzwerten der multiplen Regressionsanalyse (vgl. Backhaus et al., 2000).

Ein Maß für die Güte der Vorhersage ist das Bestimmtheitsmaß (,goodness of fit"). Dieses gibt die Güte der Anpassung der Regressionsfunktion an die empirischen Daten an (vgl. Backhaus et al., 2000). Die Schätzung der einzelnen Kriteriumswerte ist umso besser, je größer der Anteil der durch die unabhängigen Variablen (Prädiktoren) erklärten Abweichung an der Gesamtabweichung ist. Als normierte Größe nimmt es einen Wertebereich zwischen 0 und 1 an. Da das Bestimmtheitsmaß sich alternativ durch Streuungszerlegung oder als Quadrat der Korrelation $\mathrm{r}$ zwischen den beobachteten und den geschätzten Y-Werten berechnen lässt, wird es auch im Kontext der multiplen Regressionsanalyse als multipler Korrelationskoeffizient bezeichnet $r^{2}$. Das Bestimmtheitsmaß wird in seiner Höhe durch die Anzahl der Prädiktoren beeinflusst. Bei gegebener Stichprobengröße wird mit jedem hinzukommenden Prädiktor ein mehr oder weniger großer Erklärungsanteil hinzugefügt, der möglicherweise nur zufällig bedingt ist. Der Wert von $r^{2}$ kann also mit der Aufnahme zusätzlicher irrelevanter Prädiktoren dann nur zunehmen bzw. nicht abnehmen. Deshalb ist es

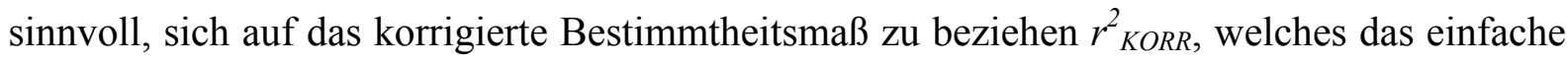
Bestimmtheitsmaß um eine Korrekturgröße vermindert, die von der Stichprobengröße abhängt und mit zunehmender Anzahl der Prädiktoren steigt. Ausgehend von dem korrigierten Bestimmtheitsmaß kann die Vorhersagegüte durch eine gezielte Auswahl besonders vorhersagerelevanter Prädiktoren noch verbessert werden. Eine geeignete Methode hierfür ist das „Schrittweise“ (,stepwise“) Vorgehen. Bei diesem Verfahren werden die unabhängigen Variablen einzeln nacheinander in die Regressionsgleichung einbezogen, wobei jeweils die Variable ausgewählt wird, die ein bestimmtes Gütekriterium maximiert. Im ersten Schritt wird diejenige Variable ausgewählt, die die höchste (positive oder negative) Korrelation mit der abhängigen Variable aufweist. In den weiteren Schritten werden die Variablen mit der höchsten partiellen Korrelation ausgewählt. Aus der Rangfolge der Aufnahme lässt sich die statistische Bedeutsamkeit der Variablen erkennen. Die Zahl der durchgeführten Aufnahmevorgänge ist variabel und hängt von der Erfüllung eines speziellen Aufnahmekriteriums ab. Eine Variable kann auch wieder aus der Regressionsgleichung entfernt werden, weil sie durch die Aufnahme anderer Variablen an Bedeutung verloren hat und das Aufnahmekriterium nicht mehr erfüllt (vgl. Backhaus et al., 2000). 


\subsubsection{Stichprobe}

Bei dieser ersten Stichprobe handelt es sich um Schülerinnen und Schüler, die bereits den 11 . Jahrgang der Oberstufe des Wirtschaftsgymnasiums besuchten und kurz zuvor eingeschult worden waren. Diese Schüler waren mittels des bisherigen schuleigenen Auswahlverfahrens ausgewählt worden. Es nahmen 90 Schülerinnen und Schüler an der Eingangsdatenerhebung teil. Im Verlauf des 11. Schuljahres verließen drei Schüler die Schule. Daher beruht die endgültige, den Berechnungen zugrunde liegende Stichprobe, auf 87 Schülern (41 Frauen, 46 Männer). Das Durchschnittsalter dieser Stichprobe beträgt 17,2 Jahre. Für insgesamt 67 Schülerinnen und Schülern lagen Informationen zu deren Herkunftsschulen vor. Die deutliche Mehrzahl dieser Schüler (ca. $60 \%$ ) hatte vor dem Wechsel auf die Oberstufe des Wirtschaftsgymnasiums eine Realschule besucht.

\subsection{Ergebnisse und Diskussion}

\subsubsection{Aufklärung von Kriterienvarianz durch die erhobenen Prädiktorvariablen}

Um entscheiden zu können, welche der Prädiktorvariablen den besten Beitrag zur Varianzaufklärung der Validitätskriterien leisten, wurden multiple Regressionsanalysen durchgeführt. Insgesamt wurden sieben verschiedene Kriterien vorhergesagt: das Lehrerurteil der Eignung, das Schülerurteil der eigenen Leistungszufriedenheit, Halbjahresnoten der Fächer Deutsch, Mathematik, Englisch, BRC (Betriebwirtschaft, Rechnungswesen und Controlling) sowie die durchschnittliche Halbjahresnote über alle Fächer (siehe Tabelle 4). Um eine optimale Varianzaufklärung zu erreichen, wurden zum Teil verschiedene Varianten bezüglich der Kombination der vorgegebenen Prädiktorvariablen erprobt. So wurde für die Prädiktorvariablen aus dem Bereich fachspezifische Vorkenntnisse neben den ermittelten Werten für die bereichsspezifischen Untertests und eines Gesamtwertes für jeden Leistungstest zusätzlich ein Wert über alle drei Gesamtwerte der Leistungstests berechnet (auch als „Gesamtleistungswert“ bezeichnet). Mitunter verbesserte eine Hinzunahme bzw. ein Weglassen dieses Gesamtwertes die Vorhersage. Für die Verfahren LMI-K und SSI-K-32 wurde zunächst - wie von den Autoren angegeben - jeweils ein Gesamtwert über alle Skalen berechnet. Da diese Werte sich für keine der Vorhersagen als relevant erwiesen, wurde die 
Bedeutsamkeit einzelner Items/ Skalen dieser Verfahren zur Vorhersage der sieben Kriterien geprüft. Schließlich wurden aus dem LMI-K die Skala Erfolgszuversicht sowie aus dem SSIK-32 einige Items der Skala Procrastination/Aufschieben ${ }^{6}$ als weitere Prädiktoren ausgewählt.

Tabelle 4: Aufgeklärte Kriteriumsvarianz durch Vorhersage mittels der Methode Schrittweise durch relevante Prädiktorvariablen.

\begin{tabular}{ll}
\hline Kriterium & Anteil der aufgeklärten Varianz an der
\end{tabular}

(geordnet anhand der Höhe der aufgeklärten Varianz) Gesamtvarianz r $_{\text {KORR }}^{2}$

Lehrereinschätzung der Eignung

.49

Leistungszufriedenheit der Schüler

Halbjahresnote Englisch

Halbjahresdurchschnittsnote aller Fächer .36

Halbjahresnote Deutsch .26

Halbjahresnote Mathematik

Halbjahresnote BRC

.08

Anmerkung. BRC bezeichnet die Abkürzung für ein Fach mit wirtschaftlichen Inhalten (Betriebswirtschaft, Rechnungswesen und Controlling). Die angegebenen Werte beruhen auf einer Stichprobenzahl von N=90 Schülern.

Wie aus Tabelle 4 ersichtlich, werden korrigierte Varianzaufklärungen bis zu $49 \%\left(r_{K O R R}^{2}\right.$ $=.49$ ) erreicht. Im Durchschnitt über alle sieben Kriterien handelt es sich um eine prozentuale Varianzaufklärung von $34 \%\left(r_{K O R R}^{2}=.34\right)$. Hierbei werden die höchsten Varianzaufklärungen für die Kriterien Lehrereinschätzung der Eignung, Leistungszufriedenheit der Schüler, Halbjahresdurchschnittsnote Englisch sowie Halbjahresdurchschnittsnote über alle Fächer erzielt. Eine im Vergleich $\mathrm{zu}$ allen anderen Kriterien deutlich niedrigere korrigierte Varianzaufklärung wurde für die Halbjahresnote BRC errechnet.

In Tabelle 5 ist einzusehen, welche konkreten Prädiktoren für die jeweiligen Kriterien nacheinander aufgenommen wurden und welches Gewicht diesen Prädiktoren bei der Vorhersage zugewiesen wurde. Am standardisierten Regressionskoeffizienten $\beta$ lässt sich in der Regressionsanalyse der Beitrag einer unabhängigen Variablen für die Erklärung oder Prognose der abhängigen Variable ablesen. Bei der multiplen Regression ist es sinnvoll, sich auf die standardisierten Regressionskoeffizienten $\beta \mathrm{zu}$ beziehen, um die Erklärungs- oder

\footnotetext{
${ }^{6}$ Es handelt sich hierbei um drei Items der Skala Aufschieben (Item 9, 36, 37 des Fragebogens Nr. 3), die sich in ihrer Kombination als vorhersagerelevant erwiesen haben. Diese kurze Skala zum Aufschieben erzielt noch eine interne Konsistenz von $\alpha=.40$.
} 
Prognosebeiträge der einzelnen unabhängigen Variablen (unabhängig von den bei der Messung der Variablen gewählten Einheiten) in ihrer Höhe miteinander vergleichen zu können (vgl. Backhaus et al. 2000).

Tabelle 5: Darstellung der Ergebnisse der multiplen Regressionsanalysen für die sieben Validitätskriterien.

\begin{tabular}{|c|c|c|c|}
\hline Kriterium & Aufnahme von Prädiktoren & $\begin{array}{l}\text { Anteil der } \\
\text { aufgeklärten } \\
\text { Varianz an der } \\
\text { Gesamtvarianz } \\
\text { für jeden } \\
\text { Schritt } \mathbf{r}^{2} \text { KORR }\end{array}$ & $\begin{array}{l}\text { Standardisierte } \\
\text { Regressionskoeffi- } \\
\text { zienten } \beta\end{array}$ \\
\hline $\begin{array}{l}\text { Lehrereinschät- } \\
\text { zung der Eignung }\end{array}$ & $\begin{array}{l}\text { Deutsch: Wortschatz } \\
\text { Englisch: Übersetzen } \\
\text { Formallogisches Denken: } \\
\text { N-Test } 2 \\
\text { Mathe: Funktion } \\
\text { Selbstregulation: } \\
\text { Skala Elaborationsstrategien } \\
\text { Selbstkonzept: Deutsch }\end{array}$ & $\begin{array}{l}.20 \\
.30 \\
.37 \\
.41 \\
.45 \\
.49\end{array}$ & $\begin{array}{l}.24 \\
.34 \\
.22 \\
.28 \\
-.21 \\
.22\end{array}$ \\
\hline $\begin{array}{l}\text { Halbjahresnote } \\
\text { Englisch }\end{array}$ & $\begin{array}{l}\text { Selbstkonzept: Englisch } \\
\text { Deutsch: Wortschatz } \\
\text { Selbstregulation: } \\
\text { Skala Elaborationsstrategien } \\
\text { Selbstkonzept: Deutsch } \\
\text { Selbstregulation: Skala } \\
\text { Tendenz zum Aufschieben } \\
\end{array}$ & $\begin{array}{l}.31 \\
.38 \\
.42 \\
.45 \\
.48\end{array}$ & $\begin{array}{c}.55 \\
.31 \\
-.23 \\
-.24 \\
.19\end{array}$ \\
\hline $\begin{array}{l}\text { Leistungszufrie- } \\
\text { denheit der } \\
\text { Schüler }\end{array}$ & $\begin{array}{l}\text { Deutsch: Wortschatz } \\
\text { Motivation: } \\
\text { Skala Erfolgszuversicht } \\
\text { Selbstregulation: } \\
\text { Skala Elaborationsstrategien } \\
\text { Motivation: } \\
\text { Skala Arbeitsvermeidung } \\
\end{array}$ & $\begin{array}{l}.19 \\
.31 \\
.37 \\
.43\end{array}$ & $\begin{array}{r}.37 \\
.30 \\
-.30 \\
.27\end{array}$ \\
\hline $\begin{array}{l}\text { Halbjahresdurch- } \\
\text { schnittsnote aller } \\
\text { Fächer }\end{array}$ & $\begin{array}{l}\text { Deutsch: Wortschatz } \\
\text { Selbstregulation: } \\
\text { Skala Elaborationsstrategien } \\
\text { Gesamtleistungsergebnisse }\end{array}$ & $\begin{array}{l}.23 \\
.31 \\
.36\end{array}$ & $\begin{array}{r}.34 \\
-.30 \\
.26\end{array}$ \\
\hline
\end{tabular}




\begin{tabular}{|l|l|c|c|}
\hline Halbjahresnote & Deutsch: Wortschatz & .20 & .35 \\
Deutsch & Gesamtleistungsergebnisse & .26 & .29 \\
\hline $\begin{array}{l}\text { Halbjahresnote } \\
\text { Mathematik }\end{array}$ & $\begin{array}{l}\text { Gesamtleistungsergebnisse } \\
\text { Selbstregulation: } \\
\text { Skala Elaborationsstrategien }\end{array}$ & .16 & .40 \\
\hline $\begin{array}{l}\text { Halbjahresnote } \\
\text { BRC }\end{array}$ & Gesamtleistungsergebnisse & .08 & $\mathbf{- . 3 0}$ \\
\hline
\end{tabular}

Anmerkung. Die Validitätskriterien sind einheitlich kodiert, so dass ein Zusammenhang im Sinne der Erwartung einen positiven standardisierten Regressionskoeffizienten erhält, ein erwartungswidriger Zusammenhang ein negatives Vorzeichen der Regressionskoeffizienten bedingt (diese Beta-Koeffizienten sind fett markiert). Erwartungswidrige Zusammenhänge traten konsistent für die Skala Elaborationsstrategien auf. Des Weiteren tritt einmalig ein negativer Zusammenhang für die Skala Selbstkonzept Deutsch zur Vorhersage der Halbjahresnote Englisch auf.

Die differenzierte Darstellung der statistisch relevanten Prädiktorvariablen in Tabelle 5 zeigt, dass für jedes Kriteriumsmaß andere Prädiktorvariablen zur Varianzaufklärung ausgewählt wurden. Für jedes Kriteriumsmaß kann somit eine andere Vorhersagegleichung erstellt werden. Die Regressionsgleichungen unterscheiden sich nicht nur in ihrer Zusammensetzung und durch die Anzahl der jeweils ausgewählten, statistisch relevanten Prädiktorvariablen, sondern auch hinsichtlich deren Gewichtung in Form der Regressionskoeffizienten. Das bedeutet, dass dieselben Prädiktorvariablen je nach Kriteriumsmaß in unterschiedlichem Ausmaß zur Vorhersage beitragen. Jedoch bleibt die Richtung des Zusammenhangs zwischen den Prädiktor- und der Kriteriumsvariablen, ausgedrückt durch das Vorzeichen der standardisierten Regressionskoeffizienten, für dieselben Prädiktorvariablen über alle Kriteriumsmaße unverändert. Eine Ausnahme bildet lediglich die Variable Selbstkonzept im Fach Deutsch (aus dem DISK-Gitter), die mit der Kriteriumsvariable Halbjahresnote in Englisch negativ korreliert. Einen konsistent erwartungswidrigen Zusammenhang weist ebenfalls die Prädiktorvariable Elaborationsstrategien (aus dem Pisa-Fragebogen zu Selbstreguliertem Lernen) bei der Vorhersage auf.

Anhand der in Tabelle 4 dargestellten Daten zeigte sich, dass die am Schuljahresanfang erhobenen kognitiven, motivationalen und selbstregulativen Persönlichkeitsmerkmale sowie Vorwissen bis zu 49 \% der Varianz in den nach einem Vierteljahr beziehungsweise am Ende des ersten Schulhalbjahres erfassten Leistungsmaßen erklären. Vergleichbare Untersuchungen, wie die Salzburger Längsschnittstudie von Sauer \& Gamsjäger (1996) erzielen 
eine Varianzaufklärung von 51\% im ersten Gymnasialjahr und $39 \%$ am Ende der 10. Jahrgangsstufe (vgl. Sauer, 2001). Im Vergleich zu diesen Befunden erreichen die Werte eine sehr zufriedenstellende Größe, zumal es sich hier um das korrigierte Bestimmtheitsmaß handelt, welches hinsichtlich seiner Höhe noch unter dem in der Literatur oft angegebenen einfachen Bestimmtheitsmaß liegt. Die Genauigkeit der Vorhersage wird von verschiedensten Einflussfaktoren beeinflusst, so zum Beispiel der Auswahl der eingehenden Prädiktoren wie auch der Güte (Reliabilität, Validität, Objektivität) dieser Prädiktoren. Die Genauigkeit der Leistungsvorhersage hängt ebenfalls von den gewählten Leistungsmaßen (Validitätskriterien) ab. Dies ist an der Schwankungsbreite der erklärten Kriteriumsvarianz sichtbar, die von $10 \%$ bis zu $49 \%$ reicht. So liegt die korrigierte Varianzaufklärung von drei Kriterien (Lehrereinschätzung der Eignung, Halbjahresnote Englisch, Leistungszufriedenheit der Schüler) im oben genannten Bereich von 49 \%. Es wird deutlich, dass sich interindividuelle Leistungsunterschiede im Eignungsurteil der Lehrer, der leistungsbezogenen Selbsteinschätzung der Schüler besser vorhersagen lassen als Leistungsunterschiede in den Halbjahresnoten. Als Ausnahme können hier die Halbjahresnote Englisch sowie die Halbjahresdurchschnittsnote gesehen werden. Für die Halbjahresnoten Deutsch, Mathematik und BRC (Betriebswirtschaft, Rechnungswesen, Controlling) wurden keine Prädiktoren aus den Bereichen Motivation und Selbstregulation, sondern nur Prädiktoren aus dem Bereich Vorwissen aufgenommen. Hieraus ist zu schließen, dass die Kriterien, die durch eine Kombination der Bereiche Vorwissen sowie Motivation und Selbstregulation erklärt werden, insgesamt besser aufgeklärt werden können als die Kriterien, die ausschließlich durch Prädiktoren des Bereichs Vorwissen vorhergesagt werden. Dies beinhaltet bereits einen Hinweis zur berechtigten Annahme der hinter der aufgestellten Fragestellung stehenden Vermutung, dass die zusätzliche Aufnahme psychologischer Verfahren die durch die Leistungstests erzielte Varianzaufklärung im Durchschnitt noch erhöht. Insbesondere das Kriterium Eignungsurteil der Lehrer ist hierbei von besonderer Relevanz. Dieses Validitätskriterium erzielt mittels der gleichmäßigen Kombination von Prädiktoren beider Bereiche die höchste Varianzaufklärung. Es ist $\mathrm{zu}$ vermuten, dass Lehrer bei dieser Einschätzung des Leistungspotentials der Schüler und der zu erwartenden Leistungsentwicklung aufgrund ihrer Berufserfahrung andere Informationen einbeziehen und diese anderweitig gewichten als das im Falle der Beurteilung durch Noten der Fall ist. So mag ein Lehrer hier vorhandene Vorkenntnisse, Motivation und Selbstregulationskompetenzen stärker betonen als dies bei der Notenvergabe möglich ist. Für die einbezogenen Halbjahresnoten sind sicherlich andere Maßstäbe relevant. Des Weiteren ist zu bedenken, dass es sich bei den einbezogenen 
Zeugnisnoten um die erste umfangreiche Leistungsbewertung nach dem ersten halben Jahr auf einer neuen Schule, in einem neuen Klassenverband, mit neuen Anforderungen handelt. $\mathrm{Zu}$ erwarten wäre, dass im weiteren Verlauf der gymnasialen Oberstufe die erhobenen Vorkenntnisse und noch stärker die Selbstregulationskompetenzen einen größeren Anteil der Schulnotenvarianz aufklären können.

Es fällt auf, dass die Aufklärung der Kriteriumsvarianz der Halbjahresnote für das Fach BRC im Vergleich zu den Noten der Fächer Deutsch, Englisch und Mathematik zu einem deutlich geringeren Ausmaß möglich ist. Dieser Effekt einer geringeren Varianzaufklärung in Nebenfächern im Vergleich zu Hauptfächern ist auch aus der Literatur bekannt (vgl. Heller, 1997). So ist in diesem Fall davon auszugehen, dass dieses Fach einen relativ eigenständigen Leistungsbereich darstellt, in dem die Schüler vermutlich weniger von ihren fachspezifischen Vorkenntnissen in den Hauptfächern profitieren können.

Zusammenfassend ist hier zu betonen, dass die prognostische Validität des neu konzipierten Diagnoseinstrumentes zu diesem Zeitpunkt als belegt gelten kann.

\section{Welche der Leistungen und „psychologischen“" Merkmale der Bereiche kognitive Grundfertigkeiten, Motivation und Selbstregulation tragen zur Aufklärung des Schulerfolges bei?}

Zur Beantwortung dieses ersten Teils der aufgestellten Fragestellung wird dargestellt, wie eine weitere Optimierung des Diagnoseinstrumentariums aufbauend auf den vorgestellten Ergebnissen aussehen kann. Diese erste Studie diente der Abschätzung der Bedeutsamkeit der verschiedenen Prädiktoren. Aus diesem Grunde wurde eine möglichst breite Auswahl von relevanten Variablen vorgenommen. Nach diesen ersten empirischen Ergebnissen können Prädiktoren identifiziert werden, die keinen beziehungsweise einen signifikanten Beitrag zur Aufklärung der Kriteriumsvarianz leisten. Die Bedeutsamkeit der ausgewählten Prädiktoren kann zum einen aufgrund der Häufigkeit der Aufnahme für die Varianzaufklärung verschiedener Kriterien, zum anderen aufgrund der Gewichte, die der Prädiktorvariable hinsichtlich der Aufklärung eines Kriteriums im Vergleich zu den anderen Prädiktoren zukommt, deskriptiv beurteilt werden. Die hierfür relevanten Informationen sind Tabelle $5 \mathrm{zu}$ entnehmen. Aus dem Bereich der Leistungstests haben sich folgende Prädiktoren als bedeutsam erwiesen: Deutsch: Wortschatz, Englisch: Übersetzen, Mathematik: Funktion, Ge- 
samtleistungsergebnisse (über alle drei Leistungstests). Zum Bereich der psychologischen Verfahren wurden die Prädiktoren: Selbstkonzept Deutsch, Selbstkonzept Englisch (DISKGitter), die Skala Elaborationsstrategien (Pisa-Fragebogen zu Selbstreguliertem Lernen), die Skala Arbeitsvermeidung (aus dem SELLMO), die Skalen Aufschieben (aus dem SSI-K-32) sowie Erfolgszuversicht (LMI-K) und der Untertest N-Test2 (aus dem KFT) ausgewählt.

Es fällt auf, dass die fachspezifischen Vorkenntnisse hier erwartungsgemäß das größte Vorhersagegewicht besitzen. Das bedeutet, dass bessere Ergebnisse in den Leistungstests mit besseren Leistungen und höherem Schulerfolg, zumindest während des ersten halben Jahres an der Schule, einhergingen. Hierbei wurden aus den drei Leistungstests die Untertests Deutsch: Wortschatz, Englisch: Übersetzen und Mathematik: Funktion aufgenommen. Des Weiteren erwies sich ein durchschnittlicher Wert, der aus den Gesamtergebnissen in den Leistungstests (Gesamtleistungsergebnisse) gebildet wurde, als für die Vorhersage bedeutsam. Aus dem Bereich kognitive Grundfertigkeiten (schlussfolgerndes Denken) erwies sich von zwei hierzu ausgewählten Tests der N-Test 2 (aus dem Kognitiven Fähigkeitstest) als kognitive Grundfertigkeit zur Vorhersage des Kriteriums Lehrereinschätzung der Eignung als relevant. Für die Vorhersage weiterer Kriterien trug diese Prädiktorvariable nicht zur Verbesserung der Prognose bei. Das mag daran liegen, dass Intelligenz einen hohen Zusammenhang zu den erbrachten Leistungen im Leistungstest aufweist und somit vermutlich zusätzlich zu den fachspezifischen Leistungen durch diese Variable keine zusätzliche Varianz aufgeklärt werden kann. Die Konzentrationsfähigkeit stand in keinem erkennbaren Zusammenhang mit den schulischen Leistungen. Hierzu kann angenommen werden, dass die Konzentrationsfähigkeit einen eher indirekten Einfluss auf die Kriterien, vermittelt über die Ergebnisse in den Leistungstests, ausübt. Aus der Literatur ist ebenfalls ein indirekter Einfluss der Konzentrationsfähigkeit über die Intelligenz auf schulische Leistungen bekannt (vgl. Schneider \& Bös, 1985). Derartige indirekte Einflüsse sind bei dem hier gewählten Vorgehen nicht aufzudecken. Ein anderer Grund für die relativ geringe Varianzaufklärung der Konzentrationsfähigkeit sowie der Intelligenz (schlussfolgerndes Denken) kann in der gewählten Form der Operationalisierung dieser Konstrukte begründet liegen. Für die Intelligenz wurde bewusst das schlussfolgernde Denken als wesentliche Komponente von Intelligenz ausgewählt. Es stellt sich allerdings die Frage, inwieweit neben nonverbalen Aspekten verbale Intelligenzleistungen ebenso einen wichtigen Einfluss auf Schulleistungsindikatoren ausüben, zumal verbale Intelligenzleistungen vermutlich in noch 
stärkerem Zusammenhang zu Schulleistungen und Noten stehen als das schlussfolgernde Denken.

Hinsichtlich des Bereichs der fächerübergreifenden Selbstregulationskompetenzen haben sich metakognitive Strategien bei der Vorhersage des Schulerfolges als nicht bedeutsam erwiesen. Es ist zu vermuten, dass es sich hier ebenfalls um eine schlecht gelungene Operationalisierung dieser kognitiven Strategien der Planung, Kontrolle und Überwachung des Lernprozesses handelt. Im Pisa-Fragebogen befindet sich hierzu die Skala Kontrollstrategien, welche allerdings eine relativ geringe interne Konsistenz $(\alpha=.64)$ aufweist. Die empirische Befundlage zu diesen Strategien ist nicht einheitlich. Probleme der Erfassung dieser Konstrukte liegen meist in der Diskrepanz zwischen der retrospektiv erinnerten und der tatsächlichen Anwendungshäufigkeit der Strategien begründet (vgl. Artelt, 2000). Aus anderen Bereichen zur Selbstregulation (kognitive Regulation, Motivationsregulation sowie Selbst- und Handlungskontrolle) konnten vorhersagerelevante Prädiktorvariablen identifiziert werden. Hier sind die Skalen Erfolgszuversicht (aus dem Leistungsmotivationsinventar-Kurzform, LMI-K), Arbeitsvermeidung (aus dem Fragebogen zur Erfassung von Lern- und Leistungsmotivation, SELLMO) sowie die Skalen Selbstkonzept Englisch und Deutsch (aus dem Verfahren zur Messung des differentiellen Selbstkonzepts schulischer Leistungen und Fähigkeiten, DISK-Gitter) zu nennen. Aus dem Selbststeuerungsinventar (SSI-K-32) wurde die Skala Tendenz zum Aufschieben zur Vorhersage aufgenommen. Hervorgehoben werden muss die Skala Elaborationsstrategien (aus dem Pisa-Fragebogen zum Selbstregulierten Lernen). Diese Variable weist eine im Vergleich zu anderen Variablen dieses Bereichs unerwartete konsistente Relevanz auf (sie erweist sich bei fünf von sieben Kriterien als relevant). Erstaunlich ist hier ebenso der konsistent negative und somit erwartungswidrige Zusammenhang elaborierten Lernens mit den Schulleistungskriterien. Schüler, die häufig Elaborationsstrategien einsetzen, weisen im Sinne dieses Zusammenhangs, schlechtere Leistungen auf und sind weniger erfolgreich in der Schule. Der Intention der Skala folgend, wurden allerdings umgekehrte Ergebnisse erwartet, insofern, dass ein hoher Einsatz von Elaborationsstrategien mit guten Leistungen einhergeht. Hierzu sind verschiedene Erklärungsansätze denkbar, die im Folgenden kurz dargestellt werden sollen. Ein erster Ansatz beinhaltet die Überlegung, dass die Angaben zum Einsatz von Lernstrategien möglicherweise nur einen geringen Zusammenhang zum tatsächlichen Einsatz dieser Strategien aufweisen. Dieser Punkt wird in der aktuellen Forschung wiederholt diskutiert (vgl. Artelt, 2000). In diesem Zusammenhang ist vorstellbar, dass „gute“ Schüler im Vergleich zu „schlechten“ Schülern vertiefende Lernstrategien möglicherweise auch 
verstärkt einsetzen, diesen Einsatz jedoch weitestgehend automatisiert haben und sich dessen weniger bewusst sind. Diese Schüler würden die Häufigkeit des Einsatzes dieser Strategien im Fragebogen unterschätzen. Im Gegensatz hierzu mag es für „schlechte“ Schüler relevanter sein, Lernstrategien einzusetzen und deren Einsatz bewusst zu üben, um ihre Leistungen zu verbessern. Diesen Überlegungen folgend wäre zu vermuten, dass diese Schüler den Strategieeinsatz überschätzen. In einem solchen Fall stellt die Automatisierung von Lernprozessen ein besonderes Problem dar, was vermutlich damit zusammen hängt, dass stärker automatisierte kognitive Prozesse dem Bewusstsein weniger zugänglich sind als kognitiv kontrollierte Prozesse (vgl. Artelt, 2000). Es ist also vorstellbar, dass „schlechte“ Schüler eher als ,gute“ Schüler auf Lernstrategien angewiesen sind und diese noch stärker bewusst einsetzen als Schüler, denen das Lernen generell leichter fällt. Verschiedene empirische Befunde unterstützen diese Vermutung. So lässt sich beispielsweise oft nur ein geringer Zusammenhang zwischen dem über Fragebogen und handlungsnah erfassten Einsatz von Lernstrategien finden (vgl. Artelt, 1999). In diesem Zusammenhang wird in der Forschung häufig auf die Notwendigkeit verwiesen, Lernstrategien im Handlungskontext zu untersuchen (vgl. Artelt, 2000). Bezogen auf das vorliegende Projekt ist allerdings zu sagen, dass eine solche Erfassung aus organisatorischen sowie ökonomischen Gründen nicht möglich war. Ein weiterer Erklärungsansatz setzt ebenfalls an der Validität der Skala an, berücksichtigt aber einen etwas anderen Sachverhalt. Es fällt auf, dass die Skala Elaborationsstrategien neben dem konsistent erwartungswidrigen Einfluss ein recht hohes Prädiktionsgewicht im Vergleich zu anderen Lernstrategien aufweist, was nicht dafür spricht, dass die Skala eine schlechte Messung der eigentlichen Lernstrategie darstellt, sondern, dass sie möglicherweise ein etwas anderes Konstrukt misst. So kann man vermuten, dass die Schüler, die hohe Werte auf dieser Skala erzielen, Probleme bei der Kontrolle und Überwachung von Lernprozessen haben und dazu neigen, durch $\mathrm{zu}$ intensive Auseinandersetzung mit dem Thema den Überblick zu verlieren und somit andere gestellte Aufgaben nicht schaffen. Insbesondere bei leistungsrelevanten Aufgaben mag sich dieser Arbeitsstil, bei dem es vermutlich an effektiven Kontrollstrategien fehlt, als ungünstig erweisen. Dieses Problem der fehlenden Zielorientierung kann in Zusammenhang mit der von Kuhl (1998) formulierten Theorie der volitionalen Handlungskontrolle gebracht werden. Personen, die einen hohen Wert auf der Skala Elaborationsstrategien erzielen, sind laut dieser Theorie dem lageorientierten Typus zuzuordnen. Lageorientierte Personen haben Schwierigkeiten, relevante Intentionen auf Zielkurs $\mathrm{zu}$ halten oder auch unrelevante Intentionen zu deaktivieren beziehungsweise auszublenden. Stattdessen neigen lageorientierte 
Personen dazu, sich besonders intensiv mit Sachverhalten auseinander zu setzen. In den Daten sind hierfür insofern Belege zu finden, dass die Skala Elaborationsstrategien einen deutlich positiven Zusammenhang mit Skalen aufweist, die Lernbereitschaft, intrinsische Motivation sowie die Bereitschaft, sich mit schwierigen Aufgaben auseinander zu setzen messen (vgl. Tabelle 18 im Anhang P).

\section{Wird durch die Hinzunahme der „psychologischen“ Merkmale noch eine Verbesserung der Prognosekraft im Vergleich zur allein durch die Leistungstests erreichten Vorhersage erzielt?}

Aus den Ausführungen des vorigen Abschnitts wurde bereits deutlich, dass sich auch aus dem Bereich der „psychologischen“ Verfahren der Bereiche kognitive Grundfertigkeiten, Motivation und Selbstregulation Variablen als bedeutsam erwiesen haben.

Wie aus der Literatur zu erwarten, wird jeweils ein großer Anteil der Varianz durch die Leistungstests aufgeklärt. Bei fünf von sieben Kriterien kann die Varianzaufklärung durch einen Einbezug psychologischer Verfahren noch gesteigert werden (siehe Abbildung 5 und Abbildung 6). Für die Kriterien Leistungszufriedenheit der Schüler und Halbjahresnote Englisch ist der Anteil, der durch die psychologischen Verfahren aufgeklärt wird, sogar noch höher als der durch die Leistungstests aufgeklärte Anteil. In der Regel wird durch dieses Vorgehen der Anteil der psychologischen Verfahren an der Gesamtvarianz etwas unterschätzt, da bei späterem Einbezug dieser Prädiktoren die zu den Leistungstests redundanten Informationen der psychologischen Verfahren nicht berücksichtigt werden. 


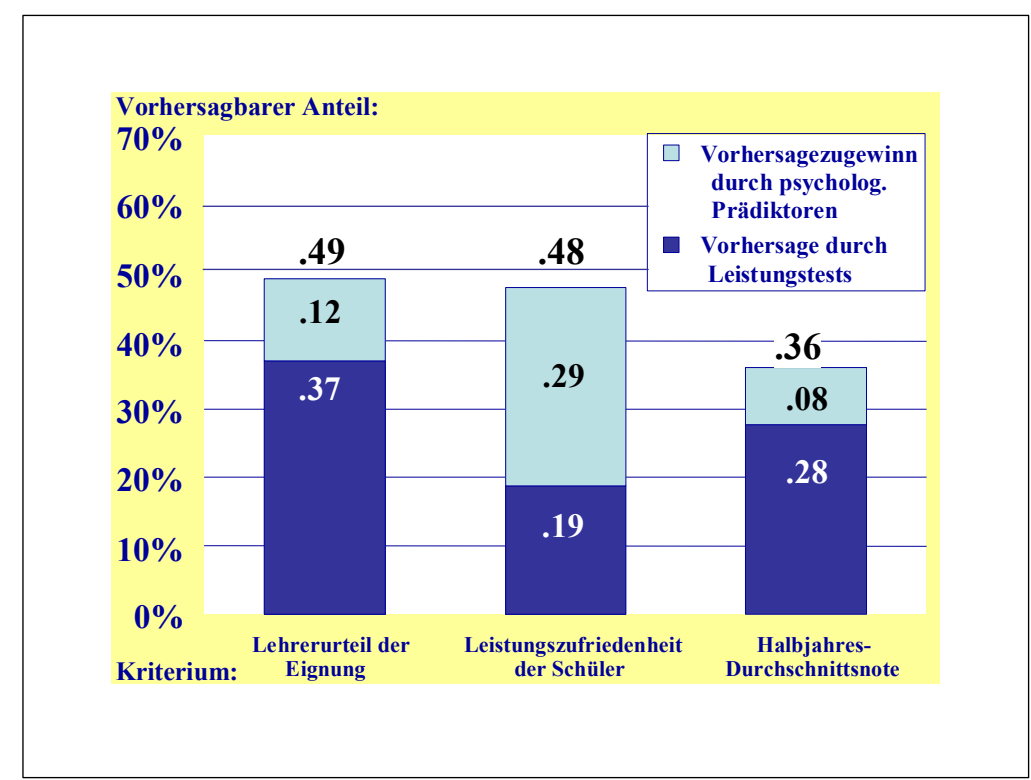

Abbildung 5: Die erzielte korrigierte Varianzaufklärung der Leistungstests sowie der Zugewinn an Kriteriumsvarianz durch die Hinzunahme der Prädiktoren aus den „psychologischen“ Verfahren für die Kriterien Lehrerurteil der Eignung, Leistungszufriedenheit der Schüler sowie Halbjahresdurchschnittsnote (N=67).

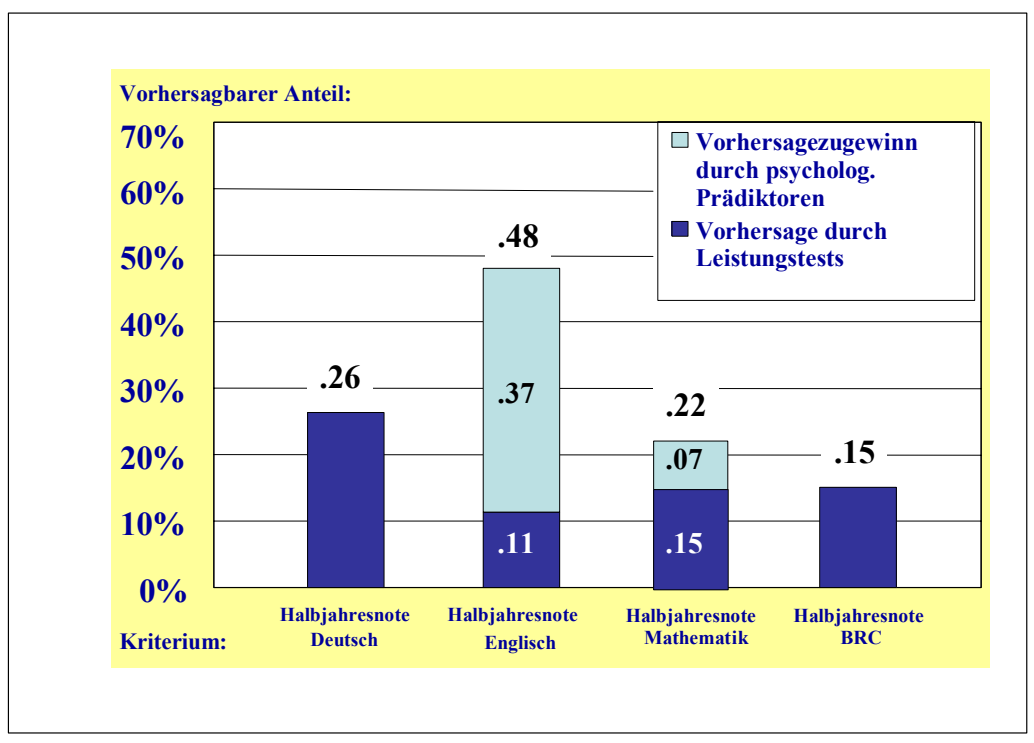

Abbildung 6: Die erzielte korrigierte Varianzaufklärung der Leistungstests sowie der Zugewinn an Kriteriumsvarianz durch die Hinzunahme der Prädiktoren aus den „psychologischen“ Verfahren für die Kriterien Halbjahresnote Deutsch, Halbjahresnote Englisch, Halbjahresnote Mathematik sowie Halbjahresnote BRC (Betriebswirtschaft, Rechnungswesen und Controlling), ( $\mathrm{N}=67)$. 
Die Ergebnisse belegen, dass ein Einbezug der psychologischen Verfahren für die meisten Kriterien zu einer Verbesserung der Varianzaufklärung führt. Im Durchschnitt wird eine Verbesserung der Varianzaufklärung von $13 \%$ erreicht. Wenn man bedenkt, dass die durch das herkömmliche Verfahren erzielte maximale Varianzaufklärung circa $10 \%$, der Durchschnitt über alle Kriterien hier 3,5 \% beträgt, dann handelt es sich hier im Durchschnitt um das Drei- bis Vierfache dessen, was durchschnittlich allein durch das ehemalige Verfahren an Varianzaufklärung geleistet wurde. Diese Ergebnisse rechtfertigen folglich die zusätzliche Aufnahme der „psychologischen“ Verfahren. Die Höhe des Zugewinns an Varianzaufklärung variiert allerdings deutlich (von 0 bis $37 \%$ ). Der höchste Zuwachs wird für die Kriterien Halbjahresnote Englisch (37 \%) und Leistungszufriedenheit der Schüler (29\%) erzielt. Geringere Werte sind für die Kriterien Lehrerurteil der Eignung, Halbjahresdurchschnittsnote sowie Halbjahresnote Mathematik zu verzeichnen. Die übrigen Kriterien (Halbjahresnote Deutsch, Halbjahresnote BRC) werden allein durch das Vorwissen aufgeklärt. Der große Anteil der Bereiche Motivation und Selbstregulationskompetenzen an der Aufklärung des Kriteriums Leistungszufriedenheit der Schüler ist durchaus nachvollziehbar. Hier fällt die theoretische Ähnlichkeit zwischen dem Kriteriumsmaß und den erhobenen Prädiktorvariablen auf. Motivationale Prädiktoren, wie die Skala Erfolgszuversicht (LMI-K), die Skala Arbeitsvermeidung (aus dem SELLMO) sowie die Skala Elaborationsstrategien (PisaFragebogen) gehen hier neben der Vorwissenskomponente Deutsch: Wortschatz in die Vorhersage ein. Die konzeptuelle Nähe der Konstrukte „Zufriedenheit mit der eigenen Leistung“ und „Motivation“ sind theoretisch durchaus begründbar. Die Relevanz verschiedener Prädiktoren aus den psychologischen Verfahren ist für das Kriterium Lehrerurteil der Eignung ebenfalls nachvollziehbar. Wie bereits an anderer Stelle erläutert, gehen in diese Einschätzung der Lehrer neben Fähigkeiten und Kenntnissen im Leistungsbereich sicherlich auch Hinweise und Informationen über persönliche Kompetenzen und Ressourcen aus den Bereichen lernrelevanter individueller Merkmale (wie kognitive Grundfertigkeiten, Motivation, Selbstregulation) ein. Auch dieser Befund stützt die Vermutung, dass die Lehrereinschätzung der Eignung Potentiale und Ressourcen der Schüler einbezieht, die sich sicherlich nicht in den konkreten Noten widerspiegeln.

Für das Validitätskriterium - Halbjahresnote Englisch - spielt das Vertrauen in die eigenen Fähigkeiten (Skala: Selbstkonzept Englisch), weniger jedoch die konkreten Leistungen im Fach Englisch eine Rolle. Hier stellt sich allerdings die Frage, inwieweit ein hohes Selbstkonzept im Fach Englisch auch mit guten Leistungen in diesem Fach zusammenhängt. Aus dem Bereich des Vorwissens ist lediglich der Untertest Deutsch: 
Wortschatz relevant. Hieran wird die konzeptuelle Ähnlichkeit beider Fächer deutlich. Die Tatsache, dass aus dem Leistungstest Englisch keine Prädiktorvariablen aufgenommen wurden, kann verschiedene Gründe haben. Beispielsweise ist denkbar, dass das Selbstkonzept Englisch hier besser zur Varianzaufklärung geeignet ist. Zum Bereich des selbstregulierten Lernens erwiesen sich die Skalen Tendenz zum Aufschieben (aus dem SSI-K-32) und Elaborationsstrategien (aus dem Pisa-Fragebogen) als bedeutsam. Der Einfluss dieser Variablen kann möglicherweise damit erklärt werden, dass für die Englischnote stärker als für andere in die Analysen einbezogene Halbjahresnoten individuelles Engagement, Mitarbeit und Fleiß wichtig sind. Dieser Umstand könnte damit zusammen hängen, dass insbesondere im Fach Englisch ein unterschiedlicher Leistungsstand der Schüler vorherrscht und dass erhöhter Fleiß und Engagement in diesem Fach - zumindest im ersten Halbjahr - stärker mit einer guten Halbjahresnote einhergehen als in anderen Fächern. Erstaunlich ist der negative Zusammenhang der Prädiktorvariable Selbstkonzept Deutsch mit der Halbjahresnote Englisch. Theoretisch würde man hier einen positiven Zusammenhang vermuten. Denn obwohl es sich um unterschiedliche Fächer handelt, weisen beide doch hinsichtlich der geforderten Fähigkeiten eine konzeptuelle Ähnlichkeit auf. Allerdings ist zu bemerken, dass der erwartungswidrige negative Einfluss dieser Variable aufgrund der relativ geringen Gewichtung keine große Rolle spielt.

Zusammenfassend kann festgestellt werden, dass die fachspezifischen Vorkenntnisse - wie aus der Literatur zu erwarten - das größte Vorhersagegewicht besitzen. Der Einbezug der Prädiktorvariablen aus den Bereichen kognitive Grundfertigkeiten, Motivation und Selbstregulation tragen zu einer durchschnittlichen Verbesserung der Varianzaufklärung der Kriterien um das Drei- bis Vierfache bei.

\subsubsection{Zur erhöhten Prognosesicherheit des Diagnoseinstrumentariums}

Um in weiteren Analyseschritten zur Beantwortung der aufgestellten Hypothese eine Vergleichbarkeit des herkömmlichen notenbasierten Verfahrens mit dem neuen testbasierten Auswahlvorgehen zu ermöglichen, wurde ebenfalls für das neue Auswahlverfahren eine Rangreihe berechnet. Hierfür wurden ausgehend von den im vorigen Abschnitt 4.3.1 dargestellten Ergebnissen zur Varianzaufklärung zwei besonders relevante Validitätskriterien ausgewählt: das Eignungsurteil sowie die durchschnittliche Halbjahresnote. Diese beiden Kriterien wurden aufgrund der im Zuge der durchgeführten Regressionsanalysen aufgestellten 
Prognosegleichungen für jeden Schüler aus den Prädiktorwerten vorhergesagt. Bevor diese zu einem Wert zusammengefasst wurden, wurden sie so umgepolt, dass sie hinsichtlich ihrer Richtung vergleichbar sind. Des Weiteren wurden beide Werte standardisiert, um eine unterschiedliche Gewichtung der beiden Kriterien zu vermeiden. Es resultierte 1 Summenwert über beide Kriterien für jeden Schüler. Diese Summenwerte wurden der Größe nach in eine Rangreihe gebracht und es wurde jedem Schüler ein Rangplatz zugewiesen. Bei gleichen Werten wurde mehrmals derselbe Rangplatz vergeben.

In der Hypothese zur erhöhten Prognosesicherheit des Diagnoseinstrumentariums wurde vermutet, dass das vorläufige Diagnoseinstrumentarium beruhend auf an Bildungsstandards orientierten Leistungstests und „psychologischen“ Verfahren der Bereiche kognitive Grundfertigkeiten, Motivation und Selbstregulation eine höhere Vorhersagekraft als das ursprüngliche notenbasierte Auswahlverfahren erzielt. Zur Beantwortung dieser Hypothese ist es nötig, dass die durch die beiden verschiedenen Verfahren erreichten Varianzaufklärungen miteinander verglichen werden. Das herkömmliche System beruhte auf den Noten der Fächer Deutsch, Mathematik und Englisch der Herkunftsschulen, deren Summe hinsichtlich verschiedener weiterer Kriterien (Schultyp, Arbeits- und Sozialverhalten, gegebenenfalls eine Empfehlung vom Klassenlehrer) relativiert wird. Hier wurde die Rangliste als Prädiktor in die Analysen aufgenommen. Um eine optimale Vergleichbarkeit zu gewährleisten, wurde ebenfalls für das Diagnoseinstrumentarium - eine Rangreihe berechnet und als Prädiktorvariable in die Vorhersage eingebracht. In Abbildung 7 und Abbildung 8 sind die erreichten korrigierten Varianzaufklärungen für beide Rangreihen im Vergleich dargestellt. In beide Berechnungen gehen Werte von 67 Schülerinnen und Schülern ein. Ursache hierfür ist, dass für die Rangreihe des herkömmlichen Verfahrens nur 67 Schüler-Daten vorlagen. Aus Gründen der Vergleichbarkeit wurden auch nur diese Schüler in die Berechnungen mit beiden Rangreihen einbezogen. Deshalb weichen die hierzu dargestellten Werte für die korrigierte Varianzaufklärung des Diagnoseinstrumentariums etwas von den in Tabelle 4 dargestellten Werten ab.

Die Abbildungen verdeutlichen, dass durch die Rangreihe, die mithilfe des entwickelten Diagnoseinstrumentariums erstellt wurde, deutlich mehr Kriteriumsvarianz aufgeklärt werden kann als durch die Rangreihe des herkömmlichen Verfahrens. Dies ist besonders eindeutig für die Validitätskriterien Lehrerurteil der Eignung, Leistungszufriedenheit der Schüler, Halbjahresdurchschnittnote sowie Halbjahresnote Deutsch. Für die restlichen Kriterien wird ebenfalls eine höhere Varianzaufklärung durch das neue Verfahren erzielt. Im Durchschnitt über alle Kriterien wird für das neue Auswahlverfahren eine um $20 \%$ 
höhere Varianzaufklärung erreicht. Diese Steigerung entspricht ungefähr dem Sechs- bis Siebenfachen der Varianzaufklärung des herkömmlichen Verfahrens.

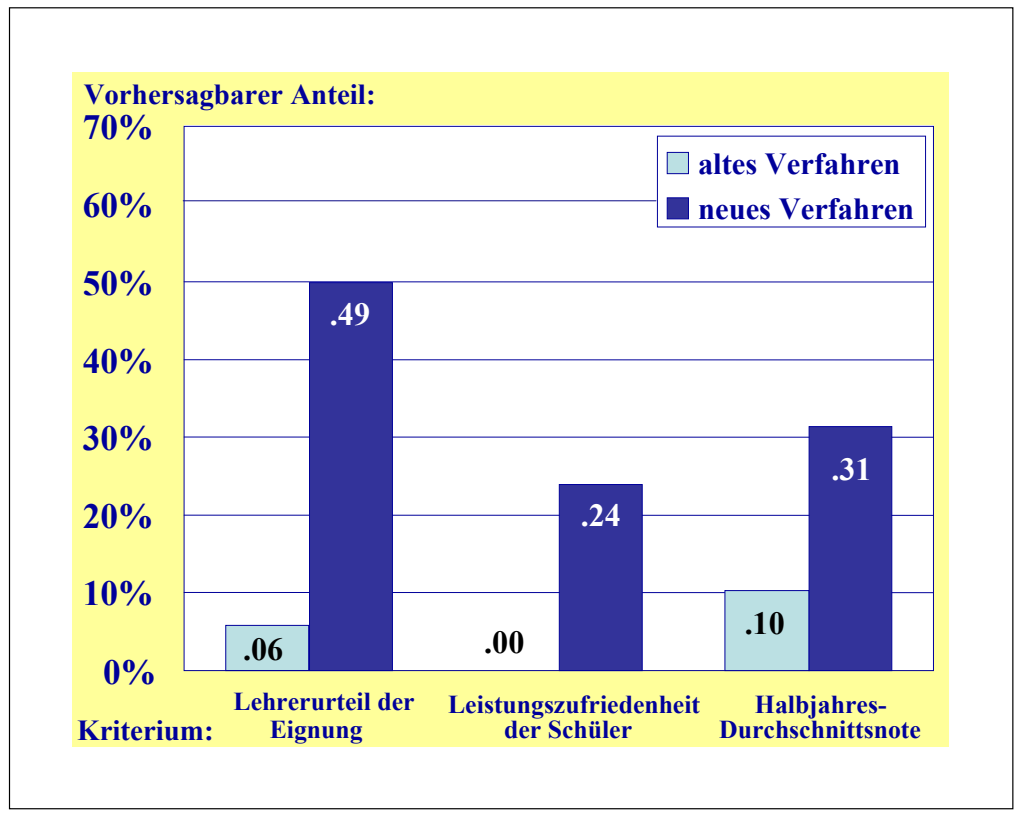

Abbildung 7: Erzielte korrigierte Varianzaufklärungen für die Kriterien Lehrerurteil der Eignung, Leistungszufriedenheit der Schüler sowie Halbjahresdurchschnittsnote der Rangreihen beider Auswahlverfahren im Vergleich $(\mathrm{N}=67)$.

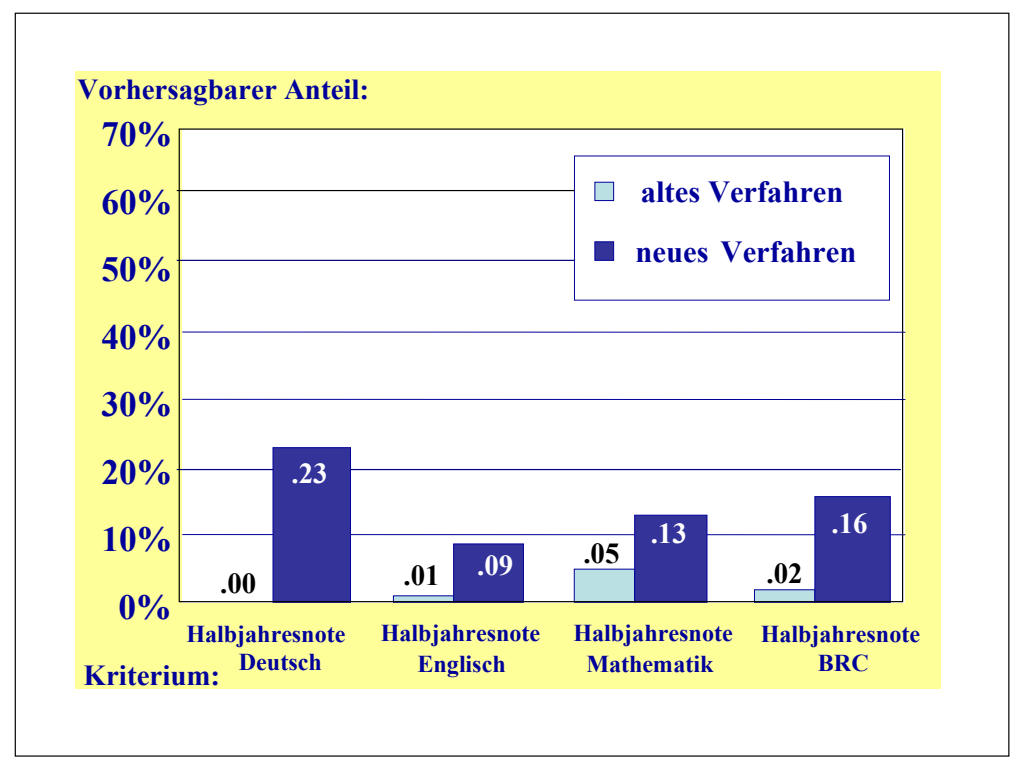

Abbildung 8: Erzielte korrigierte Varianzaufklärungen für die Kriterien Halbjahresnote Deutsch, Halbjahresnote Englisch, Halbjahresnote Mathematik sowie Halbjahresnote BRC (Betriebswirtschaft, Rechnungswesen, Controlling) der Rangreihen beider Auswahlverfahren im Vergleich $(\mathrm{N}=67)$. 
Diese Varianzaufklärungen der beiden Rangreihen für jeweils ein Validitätskriterium können statistisch auf Unterschiedlichkeit geprüft werden. Das geschieht durch Prüfung der Unterschiedlichkeit der Korrelationen, die den in den beiden Abbildungen dargestellten korrigierten Varianzaufklärungen zugrunde liegen (vgl. Bortz, 1993). Außer für die Kriterien Halbjahresnote Englisch und Halbjahresnote Mathematik unterscheiden sich alle zu den Varianzaufklärungen gehörigen Korrelationen (Spearmans rho) signifikant untereinander.

Diese Ergebnisse belegen eindrucksvoll die aufgestellte Hypothese. Anhand dieser überzeugenden Ergebnisse kann aufgrund der bisher vorliegenden Daten der Schluss gezogen werden, dass das neu konzipierte Diagnoseinstrumentarium dem herkömmlichen Auswahlverfahren hinsichtlich der prognostischen Vorhersagekraft überlegen ist.

\subsubsection{Güte der Leistungstests}

Im folgenden Abschnitt soll auf die Reliabilitäten sowie Schwierigkeiten der Leistungstests eingegangen werden. Für die Frage der Güte beziehungsweise Reliabilität des Diagnoseinstrumentariums sind hier lediglich die Werte der jeweiligen Testbausteine von Interesse, die als Bestandteile in das Instrumentarium eingehen. Dies betrifft die Untertests Englisch: Übersetzen, Deutsch: Wortschatz sowie Mathematik: Funktion sowie die Gesamttestwerte der drei Leistungstests, die zu einem Gesamtwert zusammengefasst werden.

Die Reliabilitäten wurden gemeinsam für beide Testversionen berechnet, da bei der Vorhersage der Rangreihe mittels Vorhersagegleichungen nicht zwischen beiden Testversionen unterschieden wird (vgl. Tabelle 6). Die berechneten Reliabilitäten (interne Konsistenz: Cronbachs Alpha) erreichen zufrieden stellende Werte. Eine Ausnahme bildet der Leistungstest Deutsch, welcher eine weniger zufrieden stellende Reliabilität erzielt. Eine genauere Analyse der in diesen Wert eingehenden Untertests verdeutlicht, dass es sich bei diesem Test tatsächlich nicht um einen in sich homogenen Test handelt. Dies wurde keinesfalls angestrebt. So weicht beispielsweise der Untertest Textverständnis hinsichtlich Aufbau und Inhalt deutlich von den anderen Untertests ab. Hier geht es darum, offene Fragen zu zwei kurzen Texten zu beantworten. So ist dieser Untertest in sich selbst weniger homogen als es von einem üblichen Test zu erwarten wäre ( $\alpha=.39$, über beide Testversionen). Die übrigen Untertests weisen im Vergleich zu dem Test Textverständnis eher eine klassische Testform auf, erfassen allerdings auch verschiedene im Rahmen des Faches Deutsch geforderte Fertigkeiten. Bei den Untertests Zeichensetzung und Rechtschreibung handelt es 
sich um einstudierte und geübte Regelkenntnis und deren Anwendung. Im Untertest Wortschatz ist das Sprachgefühl sowie die Kenntnis von Wörtern und deren Bedeutungen gefordert. So kann man schlussfolgern, dass sich der Gesamttest Deutsch offensichtlich aus relativ heterogenen Teilen zusammensetzt, was den erzielten niedrigen Reliabilitätswert erklärt. Trotzdem wäre es wünschenswert, diesen Test weiterhin so zu optimieren, dass er befriedigende Gütemerkmale in sich vereint.

Tabelle 6: Gütemerkmale (Reliabilität, Schwierigkeit) der relevanten Bausteine der Leistungstests (Deutsch, Englisch, Mathematik) der ersten Datenerhebung.

\begin{tabular}{|c|c|c|c|}
\hline Leistungstest & $\begin{array}{l}\text { Reliabilität } \\
\text { (interne Konsistenz) }\end{array}$ & \multicolumn{2}{|c|}{$\begin{array}{l}\text { Schwierigkeit } \\
\text { Version A/B }\end{array}$} \\
\hline \multicolumn{4}{|l|}{ Englisch } \\
\hline $\begin{array}{l}\text { Gesamt } \\
\text { Übersetzen }\end{array}$ & $\begin{array}{l}\alpha=.78 \\
\alpha=.78\end{array}$ & $\begin{array}{l}\mathrm{p}=.54 \\
\mathrm{p}=.40\end{array}$ & $\begin{array}{l}\mathrm{p}=.58 \\
\mathrm{p}=.54\end{array}$ \\
\hline \multicolumn{4}{|l|}{ Deutsch } \\
\hline $\begin{array}{l}\text { Gesamt } \\
\text { Wortschatz }\end{array}$ & $\begin{array}{l}\alpha=.43 \\
\alpha=.79\end{array}$ & \multicolumn{2}{|c|}{$\mathrm{p}=.57$} \\
\hline \multicolumn{4}{|l|}{ Mathematik } \\
\hline $\begin{array}{l}\text { Gesamt } \\
\text { Funktion }\end{array}$ & $\begin{array}{l}\alpha=.71 \\
\alpha=.75\end{array}$ & $\begin{array}{l}\mathrm{p}=.51 \\
\mathrm{p}=.47\end{array}$ & $\begin{array}{l}\mathrm{p}=.49 \\
\mathrm{p}=.44\end{array}$ \\
\hline
\end{tabular}

Anmerkung. Diese Berechnungen basieren auf einer Stichprobengröße von $\mathrm{N}=89$ bzw. $\mathrm{N}=90$ Schülerdaten. Für den Untertest Deutsch: Wortschatz wurde, aufgrund der Gleichheit beider Testversionen, eine gemeinsame Schwierigkeit für beide Testformen berechnet.

Hinsichtlich der Test-Schwierigkeiten ist vor allem wichtig, dass sich die beiden zueinander gehörenden Testformen nicht deutlich hinsichtlich ihrer Schwierigkeiten unterscheiden. Die Schwierigkeiten sind für die meisten der relevanten Testbestandteile der Leistungstests hinsichtlich ihrer Höhe ähnlich (vgl. Tabelle 6). Bei einem Vergleich der Gesamtversionen der Leistungstests ist der Test im Fach Deutsch am leichtesten, die Leistungstests Englisch und Mathematik weisen Schwierigkeiten im optimalen mittleren Bereich auf. Die Untertests Englisch: Übersetzen, Mathematik: Funktion und Deutsch: Wortschatz weisen ebenfalls mittlere Schwierigkeiten auf und sind ein wenig schwieriger als der jeweilige Gesamttest. Hierzu ist zusammenfassend $\mathrm{zu}$ sagen, dass die Forderung nach vergleichbaren Schwierigkeiten der beiden Testversionen erfüllt ist. 
Auf Aspekte der Validität der Leistungstests soll an dieser Stelle nicht weiter eingegangen werden. Da die Konstruktion der Leistungstests stark an den Bildungsstandards orientiert wurde, wird davon ausgegangen, dass durch diese strenge Orientierung an vorliegenden verbindlichen inhaltlichen Richtlinien die geforderte Inhaltsvalidität absichert ist.

\subsubsection{Fazit und weiteres Vorgehen}

Ein Blick auf die Varianzaufklärung der Validitätskriterien zeigt, dass es mittels der Daten dieser 1. Studie gelingt, maximal $49 \%$ der Kriteriumsvarianz aufzuklären. Dieses Ergebnis belegt eindeutig, dass das erstellte Diagnoseinstrumentarium eine gute Prognosekraft besitzt. Die verschiedenen Validitätskriterien variieren hier hinsichtlich der erzielten Varianzaufklärung im Bereich von $10 \%$ bis $49 \%$. Hieran wird deutlich, dass es unterschiedlich gut gelingt, die einzelnen Validitätskriterien vorherzusagen. Am besten konnten die Kriterien Eignungsurteil der Lehrer, Halbjahresnote Englisch, Einschätzung der eigenen Schülerzufriedenheit aufgeklärt werden. Ebenfalls gute Varianzaufklärungen wurden für die Kriterien durchschnittliche Halbjahresnote sowie Halbjahresnoten Deutsch und Mathematik erzielt.

An diesem Punkt stellt sich die Frage danach, welche der in das vorläufige Diagnoseinstrumentarium aufgenommenen Prädiktorvariablen zur Aufklärung von Kriteriumsvarianz beitragen. Als zentraler Bereich ist hier das Vorwissen zu nennen. Außerdem erwiesen sich Prädiktorvariablen der Bereiche Motivation und Selbstregulation als bedeutsam (vgl. Tabelle 7).

Der Einbezug psychologischer Verfahren in die Vorhersage erbrachte eine durchschnittliche Verbesserung der durch das Vorwissen erzielten Varianzaufklärung um das Drei- bis Vierfache. Einen relativ großen Anteil der durch psychologische Verfahren aufgeklärten Varianz weisen die Validitätskriterien durchschnittliche Halbjahresnote, Halbjahresnote Englisch sowie Leistungszufriedenheit der Schüler auf. Für die Kriterien Eignungsurteil, durchschnittliche Halbjahresnote und Halbjahresnote Mathematik sind diese Werte geringer. Keine zusätzliche Varianzaufkärung wird für die Kriterien Halbjahresnote Deutsch und BRC (Betriebswirtschaft, Rechnungswesen, Controlling) erzielt.

Im Kontext der hauptsächlichen Fragestellung des Projektes, ob die Schülerauswahl durch das neue Instrumentarium noch verbessert werden kann (siehe Hypothese zur erhöhten Prognosesicherheit des Diagnoseinstrumentariums) wurden Varianzaufklärungen durch die 
anhand beider Verfahren erstellten Rangreihen miteinander verglichen. Das neue testbasierte Auswahlverfahren erzielt hier eine Sechs- bis Siebenfache Varianzaufklärung im Vergleich zum ehemaligen notenbasierten Verfahren, welche bei fünf von sieben Kriterien auf signifikanten Korrelationsdifferenzen beruht.

Diese erste Studie diente unter anderem dazu, relevante und aussagekräftige Variablen für das Diagnoseinstrumentarium herauszufiltern. Hierfür wurden zur Vorhersage von sieben Kriterien multiple Regressionsanalysen durchgeführt. Aufgrund dieser Analysen und weitergehender im Diskussionsteil dargestellter Überlegungen zur Relevanz der Prädiktorvariablen wurde das Diagnoseinstrumentarium gekürzt. Hierbei wurden die Leistungstests bis auf einige kleine Optimierungen im Instrumentarium belassen. Dies liegt darin begründet, dass das Vorwissen eine große Relevanz zur Aufklärung von Schulleistungen besitzt sowie die Erhebung des Leistungsstandes und spezifischer Fähigkeiten und Fertigkeiten der Fächer Mathematik, Deutsch und Englisch ein grundlegendes Anliegen des Wirschaftsgymnasiums war. In die Vorhersagegleichungen gehen jeweils ein Untertest-Wert der Leistungstests Deutsch, Englisch, Mathematik sowie die Gesamttestwerte der Leistungstests ein. Im Rahmen der Optimierung der Leistungstests wurden diese um jeweils 5 Minuten gekürzt, so dass jeder Test innerhalb von 25 Minuten bearbeitet werden konnte. Im Fach Englisch wurde der Untertest Einsetzen aus dem Gesamttest entfernt, da dieser in der Durchführung äußerst aufwendig ist (jedem Schüler wird ein Hörtext vorgespielt). Im Fach Mathematik wurde zur Kürzung um 5 Minuten eine Teilaufgabe des Untertests Größenverhältnisse entfernt. Für den Leistungstest Deutsch wurde der Untertest Zeichensetzung aus dem Diagnoseinstrumentarium gestrichen. Da die beiden Untertests Zeichensetzung und Rechtschreibung hinsichtlich der erfragten globalen Fertigkeiten relativ ähnlich sind, sollte auf einen der beiden Tests im Weiteren verzichtet werden. Der Untertest Rechtschreibung scheint inhaltlich bezogen auf geforderte Fertigkeiten des Deutschunterrichts noch relevanter und breiter ausgerichtet als der Test zur Zeichensetzung. Des Weiteren unterscheiden sich beide Testversionen A und B des Tests Zeichensetzung deutlich hinsichtlich ihrer Reliabilität (A: $\alpha=.57$; B: $\alpha=.30$ ), was ebenfalls zur Entscheidungsfindung beitrug.

Aus den Bereichen kognitive Grundfertigkeiten, Motivation und Selbstregulation wurden die Verfahren im Instrumentarium belassen, welche sich bei mindestens einer Kriteriums-Vorhersage als relevant erwiesen haben (siehe hierzu Abschnitt 4.3.1). Zusätzlich zu diesen Prädiktorvariablen wurden vier weitere Skalen aus dem Pisa-Fragebogen zu 
selbstreguliertem Lernen vorerst im Diagnoseinstrumentarium behalten. Es handelt sich hierbei um die Skalen Wiederholungsstrategien, Kontrollstrategien, Kontrollerwartungen sowie Interesse Mathematik, die entweder aufgrund theoretischer Überlegungen einer weiteren empirischen Erprobung unterzogen werden sollten bzw. die sich in durchgeführten Voranalysen als relevant erwiesen hatten. Alle im Instrumentarium beibehaltenen Prädiktorvariablen sind in der folgenden Tabelle 7 im Überblick dargestellt.

Tabelle 7: Übersicht über die nach der ersten Optimierung im Diagnoseinstrumentarium enthaltenen Prädiktorvariablen.

\begin{tabular}{|c|c|c|}
\hline \multicolumn{2}{|c|}{ KOGNITIVE DETERMINANTEN: } & \multirow[t]{2}{*}{ SELBSTREGULIERTES LERNEN: } \\
\hline Vorwissen & $\begin{array}{l}\text { Leistungstest Deutsch } \\
\text { Leistungstest Englisch } \\
\text { Leistungstest Mathematik }\end{array}$ & \\
\hline Intelligenz & $\begin{array}{l}\text { KFT: N-Test } 2 \\
\text { (Figurenanalogien) }\end{array}$ & \\
\hline \multicolumn{2}{|c|}{ MOTIVATIONALE DETERMINANTEN: } & Motivationale Präferenzen: \\
\hline $\begin{array}{l}\text { Lern- und } \\
\text { Leistungs- } \\
\text { motivation }\end{array}$ & $\begin{array}{l}\text { LMI-K: Erfolgszuversicht } \\
\text { SELLMO: Arbeitsvermeidung }\end{array}$ & $\begin{array}{l}\text { Interesse (Mathematik) } \\
\text { Selbstbezogene Kognitionen: } \\
\text { Kontrollerwartungen }\end{array}$ \\
\hline \multicolumn{2}{|c|}{ VOLITIONALE DETERMINANTEN: } & Lernstrategien: \\
\hline \multicolumn{2}{|c|}{ Selbststeuerungsinventar/Kurzform: } & Wiederholungsstrategien \\
\hline & Procrastination/Aufschieben & Elaborationsstrategien \\
\hline METAKOG & ITIVE DETERMINANTEN: & Kontrollstrategien \\
\hline
\end{tabular}

Anmerkung. Es wurden alle Prädiktorvariablen im Instrumentarium belassen, die sich anhand empirischer Ergebnisse dieser Studie für die Aufklärung von Kriteriumsvarianz bedeutsam erwiesen haben. Die kursiv gekennzeichneten Skalen hatten sich im Rahmen der ersten Studie nicht als bedeutsam zur Aufklärung von Kriteriumsvarianz erwiesen. Sie wurden allerdings trotzdem vorerst im Instrumentarium belassen, um einer weiteren empirischen Erprobung unterzogen zu werden. Die Items der Skala Erfolgszuversicht aus dem LMI-K wurden für den Einsatz in der zweiten Studie um die darüber hinaus enthaltenen Items aus der Langform des LMI aufgestockt. 


\section{Studie 2: Validierung des Diagnose- instrumentariums}

\subsection{Fragestellung}

Aufbauend auf den Ergebnissen der ersten Studie zur Entwicklung des Diagnoseinstrumentariums sollte im Rahmen der folgenden Studie geklärt werden, inwieweit sich das entwickelte und gekürzte Verfahren zur Auswahl der Schüler im Vergleich zum ehemaligen System der Schule in der Anwendung an einer anderen Stichprobe bewährt. In diesem Schritt sollte beurteilt werden, ob das neue Vorgehen im Vergleich zum herkömmlichen notenbasierten Verfahren tatsächlich zu einer besseren Schülerauswahl führt. Es kann folgende Hypothese formuliert werden:

Hypothese zur prognostischen Überlegenheit des entwickelten Diagnoseinstrumentariums:

Das erstellte Diagnoseinstrumentarium beruhend auf an Bildungsstandards orientierten Leistungstests und „psychologischen“ Verfahren der Bereiche kognitive Grundfertigkeiten, Motivation und Selbstregulation führt zu einer besseren Auswahl geeigneter Schülerinnen und Schüler als das herkömmliche notenbasierte Auswahlverfahren.

\subsection{Methode}

\subsubsection{Ablauf der Untersuchung}

Die Datenerhebung kann analog zum Vorgehen der ersten Studie in zwei große Messzeitpunkte unterteilt werden: 1.) die Erhebung der Prädiktorvariablen sowie 2.) der Erhebung der Validitätskriterien.

Zum 1. Messzeitpunkt im März 2005 wurden die im gekürzten Diagnoseinstrumentarium enthaltenen Prädiktorvariablen erhoben. Es nahmen insgesamt 173 Schülerinnen und Schüler an der Erhebung teil. Diese hatten sich für einen Platz an der Oberstufe des Wirtschaftsgymnasiums beworben. Zur Haupterhebung im März wurde ein Großteil der Schüler $(\mathrm{N}=153)$ getestet, an einer kurz darauf folgenden Nachtestung nahmen weitere 20 
Schüler teil. Zu beiden Zeitpunkten wurde ähnlich verfahren. Die Schüler wurden jeweils in parallelen Gruppen untersucht (für die Nachtestung war dies aufgrund der geringen Gruppengröße nicht nötig). Zunächst führten Fachlehrer die Leistungstests der Fächer Mathematik, Deutsch und Englisch durch. Hierfür wurden jeweils 25 Minuten Zeit veranschlagt, die Instruktionen befanden sich auf den Testbögen. Im Anschluss daran wurden die „psychologischen“ Verfahren durchgeführt. In einem Zeitraum von 30 Minuten wurde zunächst der Test zum formallogischen Denken (N-Test 2 aus dem Kognitiven FähigkeitsTest, KFT) zum Bearbeiten vorgelegt. Anschließend wurde ein Fragebogen ausgefüllt, welcher die nach der ersten Optimierung im Diagnoseinstrumentarium verbliebenen Skalen enthielt. $\mathrm{Zu}$ den verschiedenen Verfahren wurden jeweils ausführliche Instruktionen gegeben (Instruktion zu dem Fragebogen siehe Anhang E).

Die Erhebung der Validitätskriterien fand im Dezember 2005/ Januar 2006 statt. Des Weiteren wurden im Februar 2006 durchgeführte psychometrische Leistungstests der Fächer Mathematik und Englisch sowie die im Juli 2006 vergebenen Endjahresnoten der Schüler als Validitätskriterien in die Analysen aufgenommen.

\subsubsection{Variablen}

\section{Unabhängige Variablen (Prädiktoren)}

Die Leistungstests der Fächer Deutsch, Mathematik und Englisch wurden größtenteils unverändert aus der ersten Studie übernommen. Zur Verringerung der Durchführungszeit waren anhand der empirischen Ergebnisse der ersten Studie minimale Kürzungen vorgenommen wurden, so dass die Leistungstests innerhalb von 25 Minuten durchgeführt werden konnten. Aus dem Leistungstest Deutsch war der Untertest Zeichensetzung, aus Englisch der Untertest Einsetzen und aus dem Leistungstest Mathematik eine Teilaufgabe des Untertests Größenverhältnisse eliminiert worden.

Die psychologischen Verfahren der Bereiche kognitive Grundfertigkeiten, Motivation und Selbstregulation waren aufgrund der empirischen Ergebnisse der ersten Studie soweit minimiert worden, dass diese nunmehr in der Hälfte der zuvor benötigten Zeit durchgeführt werden konnten (30 Minuten nach der Kürzung). Dabei blieben die ursprünglichen Skalen und Tests größtenteils unverändert, es wurden lediglich irrelevante Skalen und Tests aus dem Instrumentarium entfernt. 
In Tabelle 7 des vorigen Kapitels sind die Leistungstests sowie die Verfahren zur Erfassung relevanter individueller Merkmale dargestellt, die im Instrument belassen wurden und zur Erhebung eingesetzt wurden.

\section{Abhängige Variablen (Validitätskriterien)}

Zur Validierung und Optimierung des Diagnoseinstrumentariums wurden einige Monate nach der Erhebung der unabhängigen Variablen (Prädiktorvariablen) abhängige Variablen (Kriterien bzw. Validitätskriterien) erhoben. Es handelt sich hierbei um Variablen, die den Schulerfolg der Schüler möglichst gut abbilden. Hierzu gehören eine Einschätzung der Eignung der Schüler durch mehrere Fachlehrer, eine Skala zur Erfassung der eigenen Leistungszufriedenheit der Schüler sowie die Halbjahresnoten. Zusätzlich zu diesen Kriterien wurden psychometrische Leistungstests ${ }^{7}$ der Fächer Mathematik und Englisch sowie die Endjahresnoten erhoben. Diese Validitätskriterien werden im Folgenden vorgestellt.

Von vier Fachlehrern der Fächer Mathematik, Deutsch, Englisch und BRC ${ }^{8}$ wurde jeweils ein Lehrerurteil zur Eignung anhand von vier Items auf einer vierstufigen Ratingskala abgegeben (von sehr (geeignet) bis gar nicht (geeignet)), (Fragebogen siehe Anhang B). Die für jeden Schüler vorliegenden Urteile wurden zunächst für jeden Lehrer über die vier Items zusammengefasst und schließlich wurde ein Mittelwert über alle Lehrerurteile für jeden Schüler gebildet. Dieser Wert ging als Kriteriumswert in die Regressionsanalysen ein. Die Skala Eignungsurteil wurde bereits erfolgreich in der ersten Studie eingesetzt. Für diese Skala wurde aufgrund der Daten der ersten Studie eine interne Konsistenz von $\alpha=.90$ berechnet werden.

Das Validitätskriterium Leistungszufriedenheit der Schüler wird mit einem kurzen selbst entwickelten Fragebogen bestehend aus zehn Aussagen erhoben, die auf einer vierstufigen Ratingskala (von stimmt genau bis stimmt gar nicht) beantwortet werden (siehe Fragebogen im Anhang C). Als Wert für die subjektive Leistungszufriedenheit der Schüler wurde ein Mittelwert über den Fragebogen gebildet, welcher als Kriterium in die Analysen einging. Für diese Skala wurde ebenfalls anhand der Daten der ersten Studie ein Wert zur internen Konsistenz von $\alpha=.73$ berechnet.

Weiterhin wurden im Januar 2006 die Halbjahresnoten der Kernfächer Mathematik, Deutsch, Englisch und BRC (Betriebswirtschaft, Rechnungswesen und Controlling) sowie

\footnotetext{
${ }^{7}$ Hiermit soll Herrn Prof. Dr. R. Watermann und Frau Dipl. Psych. J. Warwas für die zur Verfügung gestellten Daten aus den psychometrischen Leistungstests gedankt werden.

${ }^{8}$ BRC bezeichnet ein Wirtschaftsfach, welches die Bereiche Betriebswirtschaft, Rechnungswesen und Controlling beinhaltet.
} 
eine Durchschnittsnote über alle Halbjahresnoten ${ }^{9}$ als Operationalisierung schulischer Leistungen herangezogen.

Im Juli 2006 wurden die Endjahresnoten der Schüler, die an der Testung teilgenommen hatten, erhoben. Als Validitätskriterien wurden hier - analog zu den Halbjahresnoten - die Noten der Fächer Mathematik, Deutsch, Englisch, BRC sowie eine durchschnittliche Note über alle Fächer zum Endjahr eingesetzt.

Im Februar 2006 wurden psychometrische Leistungstests der Fächer Mathematik und Englisch durchgeführt. Diese werden im Folgenden kurz vorgestellt.

In Mathematik wurden Aufgaben aus den Tests zur mathematischen Grundbildung und Oberstufenmathematik aus der dritten TIMSS-Studie (vgl. Baumert, Bos \& Lehmann, 2000) eingesetzt. Der Test zur mathematischen Grundbildung ist orientiert an den zentralen Stoffen des mathematischen Unterrichts der Mittelstufe, beansprucht aber keine curriculare Validität im strengen Sinne (vgl. Köller, Watermann, Trautwein \& Lütke, 2004). Die Testaufgaben sind in Alltagskontexte eingebunden. Der Test deckt die folgenden Inhalte ab: Zahlenverständnis, Proportionalität, Algebra sowie Messen und Schätzen. Die Aufgaben weisen unterschiedliche Antwortformate auf: es gibt Multiple Choice-Aufgaben und Aufgaben mit offenem Antwortformat. Letztere lassen sich noch einmal unterteilen in solche mit Kurzantworten (Short Answer) und solche mit erweitertem Antwortformat (Extended Response). Bei Aufgaben mit erweitertem Antwortformat sollen die Schülerinnen und Schüler beispielsweise einen Lösungsweg aufschreiben. Der Test zur Oberstufenmathematik ist an den Lerninhalten und -zielen der Sekundarstufe II orientiert. Dieser Fachleistungstest für den voruniversitären Mathematikunterricht beinhaltet Aufgaben, die entlang des Lehrstoffs voruniversitärer Fachkurse am Ende der Sekundarstufe II entwickelt wurden. Kriterien der Testkonstruktion waren fachliche Breite und Tiefe sowie die Passung zu den Lerninhalten und Lernzielen in der Sekundarstufe II. Der Test umfasst Aufgaben zu den Bereichen Zahlen, Gleichungen und Funktionen, Analysis, Geometrie, Wahrscheinlichkeitsrechnung und Statistik sowie Aussagenlogik und Beweise. Wie im Test zur mathematischen Grundbildung sind hier die Aufgabenformate Multiple Choice und offenes Antwortformat (entweder als Kurzantworten oder im erweiterten Antwortformat) vertreten. Letzteres verlangt vom Testbearbeiter zum Beispiel die Beschreibung eines Lösungsweges, eines Argumentationsprozesses oder einer Beweisführung. Da es sich hierbei um einen gut erprobten psychometrischen Leistungstest handelt, ist davon auszugehen, dass die Gütekriterien im

\footnotetext{
${ }^{9}$ Die durchschnittliche Halbjahresnote beinhaltet die Fächer BRC, Volkswirtschaft, Informatik, Deutsch, Englisch, Mathematik, Naturwissenschaft (wahlweise Biologie, Physik, Chemie), Sprache (wahlweise Französisch oder Spanisch), Religion oder Werte und Normen sowie Politik und Fachpraxis.
} 
zufrieden stellenden Ausmaß erfüllt sind. Als Validitätskriterien gingen jeweils die Summenwerte des Tests zur Oberstufenmathematik sowie zur mathematischen Grundbildung ein.

Für das Fach Englisch wurde ein Test eingesetzt, der Kompetenzen erhebt, die für ein Studium an einer englischsprachigen Universität relevant sind. Der Test beansprucht keine curriculare Validität. Der psychometrische Leistungstest im Fach Englisch stellt eine leicht veränderte Version des Test of Englisch as a Foreign Language (TOEFL) dar, welcher in den 60er Jahren vom „Educational Testing Service“ entwickelt wurde. Versionen vom „Institutional Testing Program“" werden üblicherweise zu Trainingszwecken eingesetzt. Der Test besteht aus drei Subskalen. Die erste, Hörverständnis (Listening Comprehension), soll die Fähigkeit erfassen, die gesprochene englische Sprache zu verstehen, so wie man sie üblicherweise in den USA hört (Kurzversion: 29 Items). In dem Untertest Grammatik und Orthografie (Structure and Written Expression) werden Kenntnisse in den Bereichen Rechtschreibung und Grammatik, definiert durch die nordamerikanischen Regeln, getestet (Kurzversion: 23 Items). Als drittes ist die Skala Vokabelwissen und Leseverständnis (Vocabulary and Reading Comprehension) enthalten, bei der die Fähigkeit, kurze Textpassagen zu lesen und zu verstehen, erfasst werden soll. Die Passagen sind hinsichtlich ihres Schwierigkeitsgrades an Texte angepasst, wie sie im Studium an amerikanischen Universitäten verwendet werden (Köller, et al., 2004) (Kurzversion: 28 Items). Auch für diesen psychometrischen Leistungstest wird davon ausgegangen, dass zufrieden stellende bis gute Gütekriterien zugrunde liegen. Für das Fach Englisch wurden Summenwerte der drei Untertests sowie ein Gesamtwert als Validitätskriterien verwendet.

\subsubsection{Analysestrategie für die Rangreihenbildung nach dem Diagnosein- strumentarium}

Um Aussagen über den erwarteten Schulerfolg der Schüler treffen zu können, sollten die erhobenen Daten aus dem Diagnoseinstrumentarium in einer Rangreihe integriert werden. Zur Bildung dieser Rangreihe wurde - wie im vorigen Kapitel unter Abschnitt 4.3.2 beschrieben verfahren. Hierfür wurden die beiden Validitätskriterien Eignungsurteil sowie durchschnittliche Halbjahresnote anhand der aus der ersten Studie vorliegenden Prognosegleichungen vorhergesagt. In einem zweiten Schritt wurde eines der beiden Kriterien so umgepolt, dass beide hinsichtlich ihrer Richtung vergleichbar waren. Um eine unterschiedliche Gewichtung der beiden Kriterien zu vermeiden, wurden beide Werte standardisiert. Es resultierte 1 
Summenwert über beide Kriterien für jeden Schüler. Diese Summenwerte wurden der Größe nach in eine Rangreihe gebracht und es konnte jedem Schüler ein Rangplatz zugewiesen werden. Bei gleichen Werten wurde mehrmals derselbe Rangplatz vergeben. Diese Rangreihe wurde dem Wirtschaftsgymnasium für die Auswahl ihrer Schüler zur Verfügung gestellt.

\subsubsection{Stichprobe}

Um den Vorgang der Schülerauswahl sowie die Zusammenstellung dieser Stichprobe nachvollziehen zu können, wird im Folgenden das mit der Auswahl verbundene konkrete Vorgehen beschrieben.

Für die Auswahl wurde sowohl das ehemalige notenbasierte Auswahlsystem des Wirtschaftsgymnasiums, als auch das neu entwickelte System basierend auf Auswahltests, eingesetzt. Eine Kombination beider Verfahren sollte zum einen die spätere Validierung des neuen Verfahrens in Abgrenzung zum ehemaligen Vorgehen erleichtern, zum anderen die Treffsicherheit der Auswahl erhöhen. Wie auch in den Jahren zuvor wurden von den Schülern für das ehemalige Auswahl-System relevante Daten erhoben (die Noten in Mathematik, Deutsch und Englisch der Herkunftsschulen, Angaben zum Arbeits- und Sozialverhalten, die Schulform sowie gegebenenfalls eine Empfehlung bzw. kritische Beurteilung vom Klassenlehrer). Aufgrund von Angaben der aus diesen Daten erstellten Rangliste wurden vorab 63 Aufnahme-Zusagen für die Oberstufe des Wirtschaftsgymnasiums vergeben. Diese Zusagen waren an die Bedingung einer Teilnahme am Auswahltest geknüpft. Es handelte sich hier um Schüler, die nach der Rangliste der Schule bis zu 7 Punkte erreicht hatten. Weitere 140 Schüler erhielten eine bedingte Zusage, d.h. eine Zusage wurde von den Ergebnissen im Test abhängig gemacht. 40 Schüler erhielten aufgrund ihres Punktewertes auf der Rangreihe der Schule eine sofortige Ablehnung. Aus der Gruppe von Schülern, die eine Zusage erhalten hatten, erschienen 43 (von 63) zum Test, alle anderen lehnten die Zusage ab. Aus der Gruppe mit einer bedingten Zusage sprangen 23 Schüler ab, so dass aus dieser Gruppe 117 Personen am Test teilnahmen. Weitere 5 Schüler nahmen den zweiten Testzeitpunkt war und 2 Schüler erschienen trotz Anmeldung nicht zum Test, so dass insgesamt 153 Schüler zum ersten Testzeitpunkt getestet werden konnten. Zum zweiten Testzeitpunkt erschienen insgesamt 20 Schüler. Am Auswahlverfahren nahmen also über beide Zeitpunkte hinweg 173 Schülerinnen und Schüler teil. Nach der ersten Testung wurden alle Schüler bis einschließlich Rangplatz 79 der anhand des neuen Auswahlverfahrens erstellten Rangliste aufgenommen sowie einige Schüler, die zuvor eine Zusage erhalten hatten, jedoch nicht in diesem Bereich lagen 
(insgesamt handelte es sich hierbei um 104 Schüler). Alle Schüler, die aufgrund dieser Vorgehensweise nicht ausgewählt werden konnten, wurden noch einmal individuell anhand ihrer Vornoten und der erzielten Leistungstestergebisse einer strengen Prüfung unterzogen. Aufgrund dieses Vorgehens wurden noch einige Einzelfallentscheidungen zur Aufnahme von Schülern getroffen (hier wurden weitere 29 Zusagen vergeben). Nach der Nachtestung wurden die später getesteten Schüler in die ursprüngliche Rangreihe eingegliedert und aufgrund ihres Rangplatzes wurden weitere 15 Schüler ausgewählt. Nach beiden Testungen konnten also insgesamt 148 Zusagen vergeben werden.

Bis zum Zeitpunkt der Einschulung verringerte sich diese Zahl um die Schüleranzahl, die den zugesagten Platz nicht annahm. Zur Erhebung des Kriteriums Eignungsurteil im Dezember 2005 nahmen 109 Schülerinnen und Schüler (57 Männer und 52 Frauen) teil. Im Juli 2006 zur Erhebung der Endjahresnoten lagen noch Daten von 99 Schülerinnen und Schülern vor. Das Durchschnittsalter der Schüler zur ersten Testung betrug 17,3 Jahre. Ungefähr die Hälfte der Schüler (46 \%) hatten zuvor eine Realschule besucht.

Nach diesem Vorgehen können vier Gruppen von Schülern unterschieden werden: 1) eine Gruppe, die allein aufgrund des herkömmlichen Systems ausgewählt wurde, 2) eine Gruppe, die aufgrund des neu entwickelten Vorgehens mittels Diagnoseinstrumentarium und Vorhersagegleichungen ausgewählt wurde, 3) eine Gruppe, die aufgrund beider Verfahren 4) sowie eine Gruppe, die aufgrund von Einzelfallentscheidungen (kein Verfahren) ausgewählt wurde. Die Einteilung dieser Gruppen sollte die weitere Evaluierung des neuen testbasierten im Vergleich zum herkömmlichen notenbasierten Verfahren bahnen.

\subsection{Ergebnisse und Diskussion}

Die Prädiktionskraft des neuen Auswahlverfahrens wurde im Rahmen der ersten Stichprobe bereits erprobt. Vor dem Hintergrund des globalen Ziels des Gesamtprojektes einer Verbesserung der Schülerauswahl soll es in dieser zweiten Studie primär um den Vergleich beider Auswahlverfahren hinsichtlich ihrer prognostischen Validität gehen.

Zur Klärung der in der Hypothese zur prognostischen Überlegenheit des entwickelten Diagnoseinstrumentariums aufgestellten Vermutung, dass das neue testbasierte Diagnoseinstrumentarium eine bessere Auswahl geeigneter Schülerinnen und Schüler als das ursprüngliche notenbasierte Auswahlverfahren ermöglicht, wurden nachfolgende Analysen durchgeführt. Diese beziehen sich größtenteils auf die beiden relevantesten Validitätskriterien 
Eignungsurteil und Halbjahresdurchschnittsnote, welche für die Auswahlentscheidung herangezogen wurden. In eine Analyse wurden alle vorliegenden Validitätskriterien einbezogen.

\subsubsection{Analysen zur prognostischen Überlegenheit des entwickelten Diag- noseinstrumentariums}

\section{Gruppenunterschiede hinsichtlich der Validitätskriterien Eignungsurteil und durch- schnittliche Halbjahresnote}

Den folgenden Analysen liegt die im Rahmen der Auswahlprozedur vorgenommene Gruppeneinteilung der Schüler zugrunde: (1) ausschließlich nach dem herkömmlichen notenbasierten Verfahren ausgewählt, (2) ausschließlich aufgrund des neuen testbasierten Verfahrens ausgewählt (3) aufgrund beider Verfahren ausgewählt. Ausgehend von der aufgestellten Hypothese zur prognostischen Überlegenheit des entwickelten Diagnoseinstrumentariums ist anzunehmen, dass signifikante Unterschiede zwischen den beiden Schülergruppen 1 und 2 bestehen. Dies wurde zunächst für das Kriterium Eignungsurteil anhand von Mittelwertsvergleichen überprüft (siehe Abbildung 9). Schaut man sich die Mittelwertsunterschiede der drei dargestellten Gruppen an, dann ist zu erkennen, dass das neue Verfahren (Rangreihe nach Auswahltest) hier einen höheren Wert erreicht als das herkömmliche Verfahren (Rangreihe nach Vornoten), wobei die Kombination beider Verfahren den höchsten Wert erzielt. Der Annahme der Hypothese folgend sollte ein signifikanter Unterschied zwischen dem herkömmlichen und dem neuen Verfahren bestehen. Diese Hypothese bestätigt sich anhand dieser Daten nicht. Es wurde allerdings ein tendenzieller Unterschied zwischen den Gruppen 1 und 3 gefunden $(t(47)=-1.90 ; p=.06$, $d=0.56$ ). Dieser Unterschied wird bei einer zweiseitigen Testung auf einem Alpha-Niveau von $10 \%$ signifikant. In Abbildung 9 ist dieser tendenzielle Unterschied durch ein , „<“ Zeichen dargestellt. 


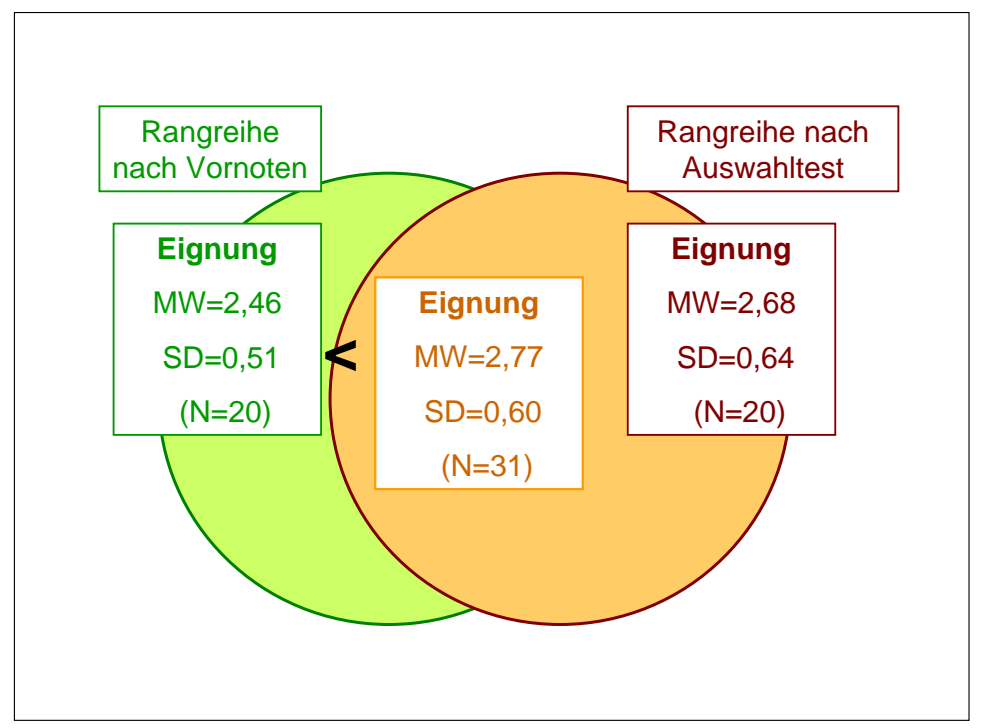

Abbildung 9: Darstellung der Mittelwerte und Standardabweichungen für das Validitätskriterium Eignungsurteil der Lehrer beider Verfahren im Vergleich hinsichtlich der drei Gruppen: (1) Auswahl nach Vornoten, (2) Auswahl nach Test sowie (3) Auswahl nach beiden Verfahren.

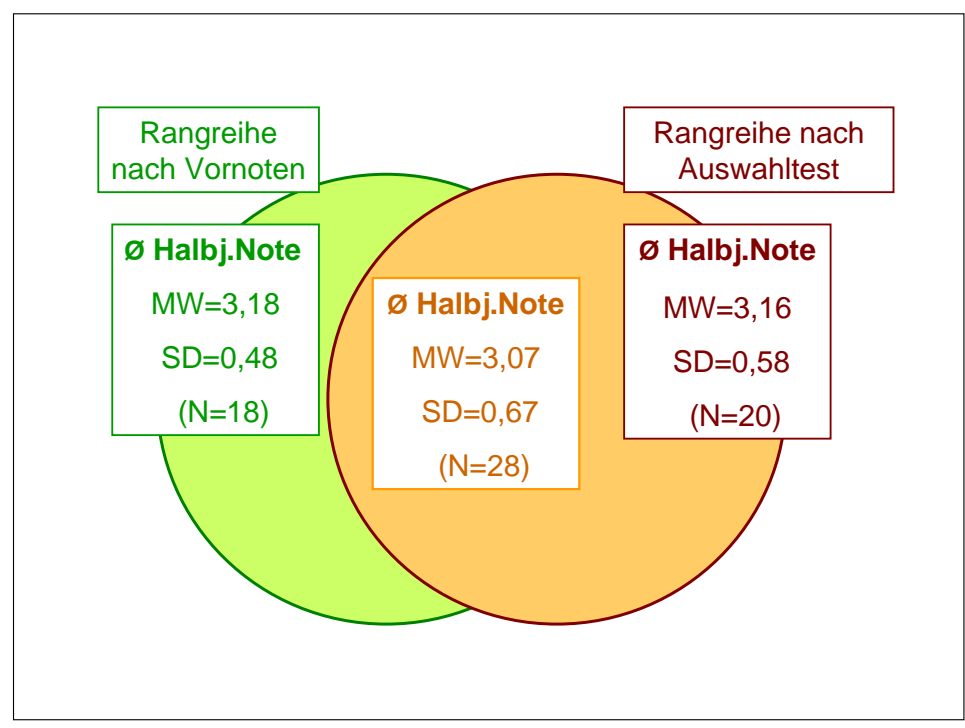

Abbildung 10: Darstellung der Mittelwerte und Standardabweichungen für das Validitätskriterium durchschnittliche Halbjahresnote beider Verfahren im Vergleich hinsichtlich der drei Gruppen: (1) Auswahl nach Vornoten, (2) Auswahl nach Test sowie (3) Auswahl nach beiden Verfahren.

Diese Ergebnisse deuten darauf hin, dass die aufgrund beider Systeme aufgenommenen Schüler von ihren Fachlehrern als geeigneter beurteilt werden als die Schüler, die allein aufgrund des herkömmlichen notenbasierten Verfahrens ausgewählt wurden. Hieran wird die 
Relevanz des neuen Diagnoseinstrumentariums insofern deutlich, dass der Einbezug von Informationen des Diagnoseinstrumentariums zu einer deutlichen Verbesserung der Auswahl der Schüler führt. Dieses Ergebnis weist auch darauf hin, dass durch das Diagnoseinstrumentarium zusätzlich neue Aspekte erfasst werden, also vermutlich eine geringe Redundanz der von beiden Verfahren erhobenen Informationen besteht.

Bei einem Vergleich der Gruppenmittelwerte dieser drei Gruppen hinsichtlich der durchschnittlichen Halbjahresnote fällt ebenfalls auf, dass die durch beide Verfahren ausgewählten Schüler eine im Durchschnitt etwas bessere durchschnittliche Halbjahresnote erzielen. Die gefundenen Unterschiede sind hier allerdings so gering, dass nicht von statistisch bedeutsamen Effekten gesprochen werden kann (siehe Abbildung 10).

Zusammenfassend kann festgehalten werden, dass ein Vergleich der Gruppenmittelwerte für das Kriterium Eignungsurteil tendenziell auf einen Vorteil der Kombination beider Auswahlverfahren im Vergleich zur alleinigen Auswahl durch das herkömmliche notenbasierte Vorgehen hinweist.

\section{Trefferquote (Sensitivität) beider Verfahren im Vergleich}

Tabelle 8: Übersicht über verschiedene Klassifikationen (tatsächlich/vorhergesagt) anhand eines bestimmten Merkmals Y in dichotomer Ausprägung ( $0=$ nicht vorhanden/negativ, $1=$ vorhanden/positiv). Diese Einteilung ermöglicht die Feststellung von Fehler- und Trefferquoten.

\begin{tabular}{c}
\begin{tabular}{c} 
Tatsächliche \\
Klassifikation \\
\cline { 2 - 4 }$(\mathrm{Y}=1)$
\end{tabular} \\
\cline { 2 - 4 }$(\mathrm{Y}=0)$
\end{tabular}


Eine weitere Analyse zur Bestimmung der prognostischen Validität beider Verfahren im Vergleich wurde mit der Bestimmung der „Trefferquote“ vorgenommen, anhand derer die Güte eines Instrumentariums beurteilt werden kann. Die Trefferquote (Sensitivität) gibt den Anteil der richtig als positiv erkannten Ergebnisse an der Gesamtheit der in Wirklichkeit positiven Ergebnisse an (siehe Tabelle 8). In die Gruppe der Personen, die von dem jeweiligen Verfahren abgelehnt wurden, gehen die Fälle ein, die von dem jeweils anderen System (herkömmliches bzw. neues Verfahren) ausgewählt wurden.

Für diese Analysen wurden die Schüler anhand der beiden relevanten Kriterien Eignungsurteil der Lehrer sowie durchschnittliche Halbjahresnote in vier Gruppen eingeteilt. Eine Dimension betraf die Aufteilung nach dem Auswahlverfahren, also die Frage nach welchem System der betreffende Schüler ausgewählt wurde. Die zweite Dimension der Aufteilung betraf das jeweilige Validitätskriterium (Eignungsurteil des Lehrers bzw. durchschnittliche Halbjahresnote). In den folgenden Tabellen ist die Aufteilung der Schüler anhand dieser Dimensionen dargestellt. Tabelle 9 zeigt die Einteilung der Schüler hinsichtlich des Kriteriums Eignungsurteil. Die Schüler mit „hoher Eignung“ erreichten auf der Skala von gar nicht geeignet (1) bis sehr geeignet (4) einen durchschnittlichen Wert von 2,5 bis 4 über die Urteile von vier Fachlehrern (Deutsch, Mathematik, Englisch und BRC). Für das neue System beträgt die Trefferquote $70 \%$, für das herkömmliche Verfahren 45 \%. Zur Erhöhung der Anzahl der Schüler in einer Gruppe wurden die Schüler in einem nächsten Schritt mit in die Analyse aufgenommen, die von beiden Verfahren ausgewählt wurden (vgl. Tabelle 10, rechter Teil). Wie hier zu sehen ist, erhöht sich die Zahl der Schüler mit hoher Eignung um 22 und die Zahl der Schüler mit niedriger Eignung um 7 für beide Verfahren. Das neue Verfahren erzielt hier immer noch eine höhere Trefferquote von $73 \%$ versus $63 \%$.

Für das Kriterium „durchschnittliche Halbjahresnote“ wurde ähnlich vorgegangen (siehe Tabelle 10). Schüler mit einer ,guten Note“ bekamen eine durchschnittliche Halbjahresnote von 3,5 und besser. Wie aus Tabelle 10 ersichtlich, ist hier die Trefferquote des neuen Verfahrens im Vergleich zum alten Verfahren besser (70\% versus 61\%), wenngleich nicht ganz so deutlich wie für das Kriterium Eignungsurteil des Lehrers. Bei einer Hinzunahme der Schüler, die nach beiden Verfahren ausgewählt wurden (rechter Teil der Tabelle), verringert sich der Abstand beider Verfahren noch etwas (73\% versus 70\%).

Die durchgeführten Analysen zur Trefferquote beider Verfahren weisen auf einen Vorteil des neuen Auswahlverfahrens im Sinne der aufgestellten Hypothese hin. Dies wird insbesondere für das Kriterium Eignungsurteil der Lehrer deutlich. Die gefundenen Unterschiede können 
allerdings im Rahmen dieser Analysestrategie nicht statistisch abgesichert werden, sondern sind als Tendenz zu betrachten.

Tabelle 9: Trefferquote (Sensitivität) für das Validitätskriterium Eignungsurteil der Lehrer. Im linken Teil der Tabelle ist diese für die Schülergruppen, die nur aufgrund jeweils eines Verfahrens ausgewählt wurden, dargestellt. Im rechten Teil der Tabelle beinhalten die Kategorien „Auswahl nach neuen Verfahren“ sowie „Auswahl nach altem Verfahren“ ebenfalls jeweils die Gruppen der Schüler, die nach beiden Verfahren ausgewählt wurden.

\begin{tabular}{|c|c|c|}
\hline & $\begin{array}{l}\text { Auswahl } \\
\text { nach neuem } \\
\text { Verfahren }\end{array}$ & $\begin{array}{l}\text { Auswahl } \\
\text { nach altem } \\
\text { Verfahren }\end{array}$ \\
\hline hohe Eignung & 14 & 9 \\
\hline $\begin{array}{l}\text { niedrige } \\
\text { Eignung }\end{array}$ & 6 & 11 \\
\hline Trefferquote & $70 \%$ & $45 \%$ \\
\hline
\end{tabular}

\begin{tabular}{|c|c|}
$\begin{array}{l}\text { Auswahl } \\
\text { nach neuem } \\
\text { Verfahren }\end{array}$ & $\begin{array}{l}\text { Auswahl } \\
\text { nach altem } \\
\text { Verfahren }\end{array}$ \\
\hline 36 & 31 \\
\hline 13 & 18 \\
\hline $73 \%$ & $63 \%$
\end{tabular}

Tabelle 10: Trefferquote (Sensitivität) für das Validitätskriterium durchschnittliche Halbjahresnote. Im linken Teil der Tabelle ist diese für die Schülergruppen, die nur aufgrund jeweils eines Verfahrens ausgewählt wurden, dargestellt. Im rechten Teil der Tabelle beinhalten die Kategorien „Auswahl nach neuen Verfahren“ sowie „Auswahl nach altem Verfahren“ ebenfalls jeweils die Gruppen der Schüler, die nach beiden Verfahren ausgewählt wurden.

\begin{tabular}{l|c|c|} 
& $\begin{array}{l}\text { Auswahl } \\
\text { nach neuem } \\
\text { Verfahren }\end{array}$ & $\begin{array}{l}\text { Auswahl } \\
\text { nach altem } \\
\text { Verfahren }\end{array}$ \\
\hline gute Note & 14 & 11 \\
\hline $\begin{array}{l}\text { schlechte } \\
\text { Note }\end{array}$ & 6 & 7 \\
\hline \multicolumn{1}{l}{ Trefferquote } & $70 \%$ & $61 \%$
\end{tabular}

\begin{tabular}{|c|c|}
$\begin{array}{l}\text { Auswahl } \\
\text { nach neuem } \\
\text { Verfahren }\end{array}$ & $\begin{array}{l}\text { Auswahl } \\
\text { nach altem } \\
\text { Verfahren }\end{array}$ \\
\hline 35 & 32 \\
\hline 13 & 14 \\
\hline $73 \%$ & $70 \%$
\end{tabular}




\section{Sensitivität und Spezifität: ROC-Kurven beider Verfahren im Vergleich}

Die Beurteilung der Schülerauswahl mithilfe einer ROC-Kurve (engl. Receiver Operating Characteristic-Kurve) stellt eine Methode dar, die im Gegensatz zur Trefferquote sowohl die Sensitivität, als auch die Spezifität der beiden Verfahren berücksichtigt. Im Gegensatz zum Vorgehen für die Bestimmung der Trefferquote bezieht diese Methode die konkret erzielten Rangreihenwerte der beiden Auswahlverfahren ein. In diese ROC-Kurve gehen also der Rangplatz des jeweiligen Auswahlverfahrens sowie dichotome Werte des betreffenden Kriteriums ein (Eignungsurteil bzw. durchschnittliche Halbjahresnote). Das Ziel im Rahmen dieser Analysestrategie ist also, festzustellen, ob das jeweilige Verfahren tatsächlich die Fähigkeit hat, zwischen den beiden Merkmalsausprägungen „Kriteriumswert positiv“ und „Kriteriumswert negativ“" zu unterscheiden.

Mittels dieses Verfahrens wird die Sensitivität und zusätzlich das Gütekriterium Spezifität berücksichtigt. Die Spezifität gibt den Anteil der richtig als negativ erkannten Ergebnisse an der Gesamtheit der in Wirklichkeit negativen Ergebnisse an (siehe Tabelle 8). Die ROC-Kurve trägt die Sensitivität auf der y-Achse und 1-Spezifität auf der x-Achse gegeneinander ab. Ein Maß für die Güte des Tests wird durch die Fläche unter der ROCKurve angegeben. Die Fläche kann Werte zwischen 0.5 und 1 annehmen, wobei ein höherer Wert die bessere Güte anzeigt. Die beste Modellgüte wird bei hoher Sensitivität und hoher Spezifität erreicht. Entspricht die Kurve der in den Abbildungen dargestellten Winkelhalbierenden, dann unterscheidet sich die Entscheidung nicht von einer Zufallsentscheidung.

Im Folgenden sind die ROC-Kurven sowie zugehörige relevante statistische Kenngrößen dargestellt. Zunächst wurden beide Verfahren anhand des Kriteriums Eignungsurteil der Lehrer verglichen (vgl. Abbildung 11, Tabelle 11). Für diese Analysen wurden die gleichen Kategorien wie für die Darstellung der Trefferquote verwendet (hohe Eignung: 2,5 bis 4 auf der Eignungsskala; niedrige Eignung: 1 bis 2,49 auf der Eignungsskala). Aus der Abbildung 11 wird deutlich, dass das neu entwickelte Verfahren besser abschneidet als das herkömmliche Verfahren. Die Kurve für dieses Verfahren liegt deutlich über der im Koordinatensystem dargestellten Winkelhalbierenden. Im Vergleich hierzu schneidet das bisherige Verfahren schlechter ab. Dies wird auch angesichts der Werte zur Fläche unter der Kurve deutlich, welche für das neu entwickelte Diagnoseinstrumentarium 0.63 versus 0.46 für das herkömmliche Verfahren der Schule betragen. Diese beiden Werte der Flächen unterscheiden sich signifikant voneinander, dies ist ersichtlich, wenn man die 
Konfidenzintervalle dieser betrachtet, die den Flächenwert der jeweils anderen Kurve nicht einschließen. Es fällt außerdem auf, dass die ROC-Kurve für das herkömmliche Auswahlverfahren knapp nicht signifikant wird (siehe Asymptotische Signifikanz: $p=.57$; $\alpha=.05)$.

ROC-Kurve

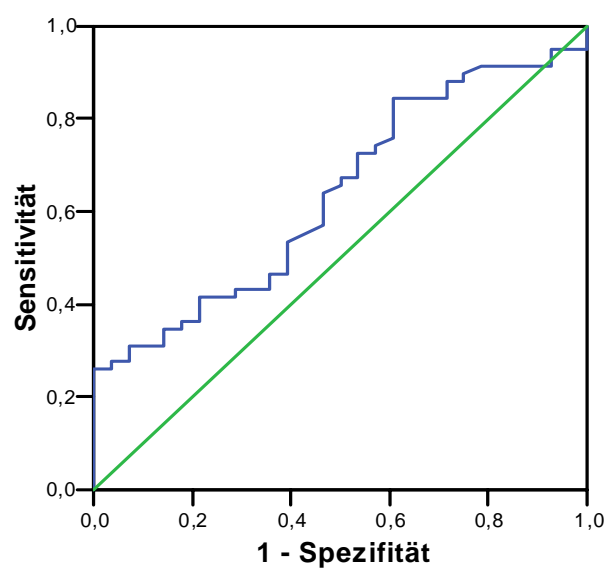

ROC-Kurve

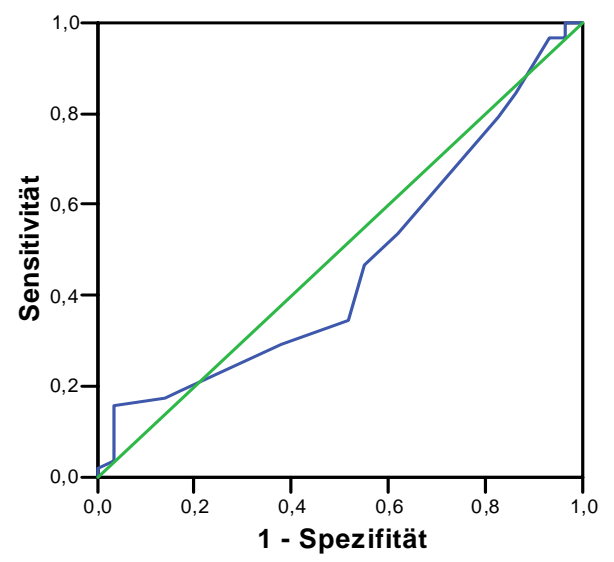

Abbildung 11: Darstellung der Sensitivität und 1-Spezifität in einer ROC-Kurve für das Kriterium Eignungsurteil. Im linken Teil der Abbildung ist dies für das testbasierte Auswahlverfahren nach dem neu entwickelten Diagnoseinstrumentarium, im rechten Teil der Abbildung für das herkömmliche notenbasierte Verfahren dargestellt.

Tabelle 11: Statistische Kennwerte der in Abbildung 11 dargestellten ROC-Kurven für das Kriterium Eignungsurteil. Im linken Teil der Abbildung ist dies für das testbasierte Auswahlverfahren nach dem neu entwickelten Diagnoseinstrumentarium, im rechten Teil der Abbildung für das herkömmliche notenbasierte Verfahren dargestellt.

\begin{tabular}{|c|c|c|c|}
\hline \multirow{2}{*}{ Fläche } & $\begin{array}{l}\text { Asympto- } \\
\text { tische } \\
\end{array}$ & Signifikanz & \multicolumn{2}{|l|}{$\begin{array}{l}\text { Asymptotisches } \\
\text { Konfidenzintervall 95\% }\end{array}$} \\
\cline { 3 - 4 } & & Untergrenze & Obergrenze \\
\hline, 635 &, 043 &, 515 &, 756 \\
\hline
\end{tabular}

\begin{tabular}{|c|c|c|c|}
\hline \multirow{2}{*}{ Fläche } & $\begin{array}{l}\text { Asympto- } \\
\text { tische } \\
\text { Signifikanz }\end{array}$ & \multicolumn{2}{|l|}{$\begin{array}{l}\text { Asymptotisches } \\
\text { Konfidenzintervall 95\% }\end{array}$} \\
\cline { 3 - 4 } & & Untergrenze & Obergrenze \\
\hline, 462 &, 567 &, 333 &, 591 \\
\hline
\end{tabular}

In einem zweiten Schritt wurden beide Verfahren hinsichtlich des Kriteriums durchschnittliche Halbjahresnote gegenübergestellt. Auch hier ist ersichtlich, dass das neu entwickelte Verfahren dem ehemaligen Verfahren überlegen ist (vgl. Abbildung 12, Tabelle 12). Die Differenz der Flächen deutet ebenfalls darauf hin, allerdings ist der Unterschied geringer als für das Kriterium Eignungsurteil der Lehrer. Die ROC-Kurven und deren 
Flächenwerte unterscheiden sich zwar augenscheinlich, doch unter Betrachtung der Konfidenzintervalle der Flächen kann statistisch nicht von einem signifikanten Unterschied ausgegangen werden. Allerdings ist der Überlappungsbereich dieser Intervalle sehr gering. Auf jeden Fall ist hier ebenfalls eine klare Tendenz zum Vorteil des neuen Auswahlverfahrens sichtbar. Des Weiteren erreicht keiner der beiden Werte für die Fläche unter den Kurven das traditionelle Signifikanzniveau von $\alpha=.05$. Bei einer Erweiterung des Signifikanzniveaus auf $\alpha=.10$ erreicht die Fläche der ROC-Kurve für das neue Verfahren Signifikanz.

ROC-Kurve

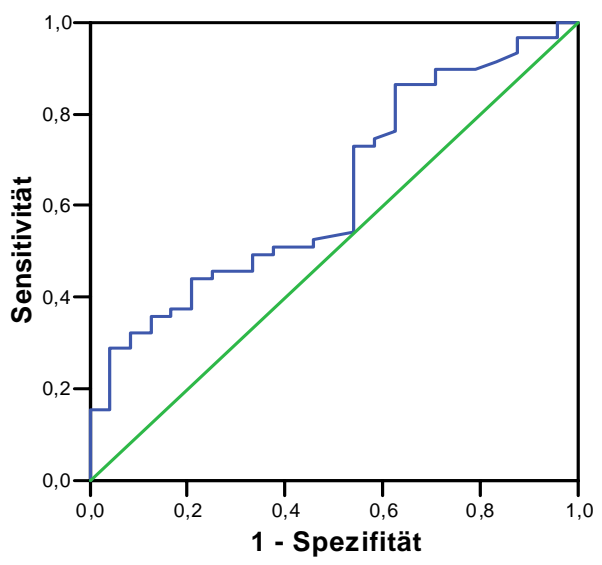

ROC-Kurve

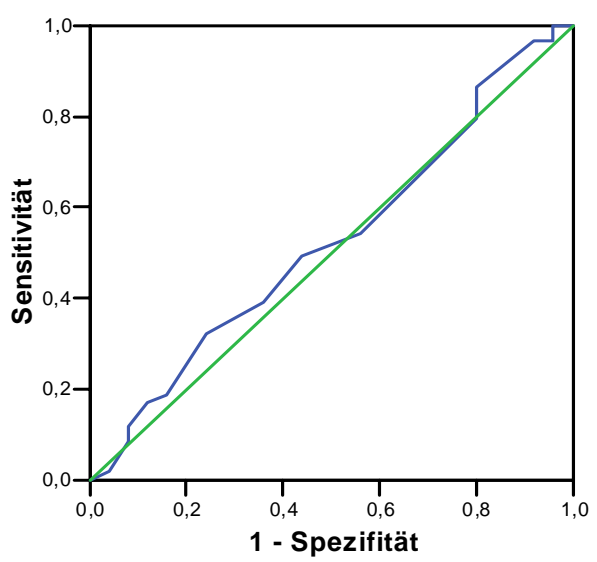

Abbildung 12: Darstellung der Sensitivität und 1-Spezifität in einer ROC-Kurve für das Kriterium durchschnittliche Halbjahresnote. Im linken Teil der Abbildung ist dies für das testbasierte Auswahlverfahren nach dem neu entwickelten Diagnoseinstrumentarium, im rechten Teil der Abbildung für das herkömmliche notenbasierte Verfahren dargestellt.

Tabelle 12: Statistische Kennwerte der in Abbildung 12 dargestellten ROC-Kurven für das Kriterium Eignungsurteil. Im linken Teil der Abbildung ist dies für das testbasierte Auswahlverfahren nach dem neu entwickelten Diagnoseinstrumentarium, im rechten Teil der Abbildung für das herkömmliche notenbasierte Verfahren dargestellt.

\begin{tabular}{|c|c|c|c|}
\hline \multirow{2}{*}{ Fläche } & $\begin{array}{l}\text { Asympto- } \\
\text { tische } \\
\text { Signifikanz }\end{array}$ & \multicolumn{2}{|l|}{$\begin{array}{l}\text { Asymptotisches } \\
\text { Konfidenzintervall 95\% }\end{array}$} \\
\cline { 3 - 4 } & & Untergrenze & Obergrenze \\
\hline, 633 &, 058 &, 505 &, 761 \\
\hline
\end{tabular}

\begin{tabular}{|c|c|c|c|}
\hline \multirow{2}{*}{ Fläche } & $\begin{array}{l}\text { Asympto- } \\
\text { tische } \\
\text { Signifikanz }\end{array}$ & \multicolumn{2}{|l|}{$\begin{array}{l}\text { Asymptotisches } \\
\text { Konfidenzintervall 95\% }\end{array}$} \\
\cline { 3 - 4 } & & Untergrenze & Obergrenze \\
\hline, 525 &, 721 &, 389 &, 660 \\
\hline
\end{tabular}

Für beide Kriterien ist ein Vorteil des neuen testbasierten Verfahrens im Vergleich zum herkömmlichen notenbasierten Vorgehen sichtbar, welcher für das Kriterium Eignungsurteil statistisch abgesichert werden kann. Hinsichtlich des Validitätskriteriums durchschnittliche Halbjahresnote wurde ein tendenzieller Unterschied beider Verfahren gefunden. Der 
signifikante Vorteil des neuen Verfahrens hinsichtlich des Validitätskriteriums Eignungsurteil der Lehrer bestätigt die aufgestellte Hypothese zur erhöhten Prognosesicherheit des Diagnoseinstrumentariums.

\section{Korrelation der Rangreihen mit den Validitätskriterien}

In die nachfolgenden Analysen wurden alle für diese Stichprobe vorliegenden Validitätskriterien einbezogen. In diese Analysen gingen die zusätzlich im Februar 2006 erhobenen Validitätskriterien aus den psychometrischen Leistungstests Englisch und Mathematik sowie die im Juli 2006 vergebenen Endjahresnoten ein (siehe Tabelle 13).

Tabelle 13: Korrelationen (Spearmans rho) der Rangreihen beider Auswahlverfahren mit verschiedenen Validitätskriterien.

\begin{tabular}{|c|c|c|c|}
\hline Validitätskriterien & $\begin{array}{c}\text { Rangreihe } \\
\text { herkömmliches } \\
\text { Verfahren }\end{array}$ & $\begin{array}{l}\text { Rangreihe } \\
\text { neues } \\
\text { Verfahren }\end{array}$ & $\begin{array}{l}\text { Differenz der } \\
\text { Korrelationen }\end{array}$ \\
\hline Eignungsurteil der Lehrer & .00 & $.18^{\mathrm{a}}$ & n.s. \\
\hline Halbjahresdurchschnittsnote & $.18^{\mathrm{a}}$ & $.21^{\mathrm{a}}$ & n.s. \\
\hline Schülerzufriedenheit & -.01 & .13 & n.s. \\
\hline Halbjahresnote Deutsch & .14 & $.37 * *$ & n.s. \\
\hline Halbjahresnote Englisch & $.22 *$ & $.23 *$ & n.s. \\
\hline Halbjahresnote Mathematik & .15 & .04 & n.s. \\
\hline Halbjahresnote BRC & .01 & .09 & n.s. \\
\hline Endjahresdurchschnittsnote & $.20^{\mathrm{a}}$ & .12 & n.s. \\
\hline Endjahresnote Deutsch & $.25 *$ & $.21^{\mathrm{a}}$ & n.s. \\
\hline Endjahresnote Englisch & .19 & .18 & n.s. \\
\hline Endjahresnote Mathematik & $.21^{\mathrm{a}}$ & -.13 & $*$ \\
\hline Endjahresnote BRC & .07 & .08 & n.s. \\
\hline
\end{tabular}




\begin{tabular}{|l|c|c|c|}
\hline Mathematik Grundbildung & -.03 & $\mathbf{. 0 0}^{\mathbf{a}}$ & n.s. \\
\hline Mathematik Oberstufe & $\mathbf{. 1 9}^{\mathbf{a}}$ & $\mathbf{. 3 8 * *}$ & n.s. \\
\hline Englisch Hörverständnis & .09 & $\mathbf{. 3 7 * *}$ & $\mathbf{a}$ \\
\hline $\begin{array}{l}\text { Englisch Grammatik und } \\
\text { Orthographie }\end{array}$ & .03 & $\mathbf{. 2 2}^{\mathbf{a}}$ & n.s. \\
\hline $\begin{array}{l}\text { Englisch Vokabelwissen u. } \\
\text { Leseverständnis }\end{array}$ & -.02 & $\mathbf{. 3 4} * *$ & $*$ \\
\hline Englisch Gesamtwert & .03 &. $\mathbf{3 7} * *$ & $*$ \\
\hline
\end{tabular}

Anmerkung. Die Stichprobengröße variiert für die verschiedenen Korrelationen zwischen 76 und 88 Schülern. Die Richtung der Korrelation ist hier jeweils so angegeben, dass die Korrelationen im Sinne der Erwartung einen positiven, die erwartungswidrigen Korrelationen einen negativen Zusammenhang aufweisen. Die signifikanten Korrelationen sind dementsprechend markiert (a $\mathrm{p} \leq .10, * \mathrm{p} \leq .05, * * \mathrm{p} \leq .01)$. Die dunkel markierten Felder kennzeichnen Korrelationen, die auf dem Alpha-Niveau von $1 \%$ sowie $5 \%$ signifikant werden. In der vierten Spalte ist angegeben, ob die Differenz der Korrelationen der beiden Auswahlverfahren für jeweils ein Kriterium sich signifikant voneinander unterscheiden (a $\mathrm{p} \leq .10, * \mathrm{p} \leq .05, * * \mathrm{p} \leq .01$, n.s. $=$ nicht signifikant).

Den folgenden Auswertungen liegen Korrelationen (Spearmans rho) der durch die beiden Auswahlverfahren erstellten Rangreihen mit allen verfügbaren Validitätskriterien zugrunde (vgl. Tabelle 13). Betrachtet man nur die signifikanten Korrelationen (auf dem 1\%, 5 \% und $10 \%$ - Signifikanzniveau $)^{10}$ beider Verfahren im Vergleich, dann ist ein Vorteil zugunsten des neuen testbasierten Verfahrens sichtbar. Eine Aussage zur Unterschiedlichkeit zweier Korrelationen ist allerdings erst möglich, wenn man die Korrelationen statistisch auf ihre Unterschiedlichkeit prüft (siehe 4. Spalte).

Die Validitätskriterien können in drei Gruppen eingeteilt werden: 1) die Halbjahresnoten sowie das Eignungsurteil der Lehrer und die eigene Leistungszufriedenheit der Schüler, 2) die Endjahresnoten sowie 3) die Werte aus den psychometrischen Leistungstests. Hinsichtlich der Halbjahresnoten (sowie des Eignungs- und Zufriedenheitsurteils) ist ein tendenzieller Vorteil des neuen Verfahrens sichtbar, welcher allerdings nicht auf signifikanten Unterschieden der Korrelationen beruht. Bezüglich der Endjahresnoten ist ein tendenzieller Vorteil des herkömmlichen Verfahrens zu sehen, welcher nur hinsichtlich der Endjahresnote Mathematik auf einer signifikanten Korrelationsdifferenz beruht.

\footnotetext{
${ }^{10}$ Eine Erweiterung des Signifikanzniveaus auf $10 \%$ wurde hier aufgrund der relativ geringen Stichprobengröße vorgenommen.
} 
Betrachtet man die signifikanten Unterschiede für die psychometrischen Leistungstests, dann zeigt sich hieran der Vorteil des neuen Verfahrens gegenüber dem herkömmlichen Verfahren.

Zusammenfassend ist aufgrund der dargestellten Ergebnisse der Vorsprung des neuen Auswahlverfahrens zu betonen, da hier vor allem signifikante Vorteile bezüglich der psychometrischen Leistungstests bestehen. Hinsichtlich der anderen Validitätskriterien ist kein eindeutiger Vorteil eines der beiden Verfahren zu verzeichnen, hier kann man lediglich von Tendenzen sprechen. Übertragen bedeuten diese Ergebnisse, dass es mit dem neuen Verfahren besser als mit dem herkömmlichen Auswahlverfahren gelingt, relevante Schulerfolgskriterien vorherzusagen, insbesondere den Erfolg im Bearbeiten von Leistungstests (bezogen auf die Fächer Mathematik und Englisch). Die Vorhersage der Kriterien Halbjahresnote Deutsch, Englisch, Halbjahresdurchschnittsnote sowie des Eignungsurteils ist tendenziell besser durch das neue Verfahren vorhersagbar. Die Kriterien Endjahresdurchschnittsnote und Endjahresnote Deutsch sind tendenziell besser und die Endjahresnote Mathematik signifikant besser durch das herkömmliche Verfahren vorhersagbar. Auch aufgrund dieser Ergebnisse kann der Schluss gezogen werden, dass sich beide Verfahren möglicherweise gut ergänzen, da sie offensichtlich auf unterschiedliche Bereiche abzielen.

Eine Zusammenfassung der unter Abschnitt 5.3.1 dargestellten Analysen spricht für die Annahme der Hypothese zur prognostischen Überlegenheit des entwickelten Diagnoseinstrumentariums. Die Ergebnisse hinsichtlich der Sensitivität und Spezifität der Auswahl durch beide Verfahren zeigen einen tendenziellen, für das Kriterium Eignungsurteil deutlichen bis signifikanten Vorsprung des neuen testbasierten Verfahrens. Die Korrelationen beider Rangreihen mit den Validitätskriterien belegen ebenfalls einen signifikanten Vorteil des neuen Verfahrens.

\subsubsection{Varianzaufklärung der Validitätskriterien}

Analog zum Vorgehen in der ersten Studie wurden multiple Regressionsanalysen zur Vorhersage der Validitätskriterien durchgeführt. Zur Vorhersage der Kriterien dienten die im Rahmen der ersten Studie aufgestellten Prädiktorgleichungen. Diese Varianzaufklärungen sind in Tabelle 14 dargestellt. Die erzielten Werte an erklärter Kriteriumsvarianz erreichen eine maximale Höhe von 13 \% für die Halbjahresnote Deutsch und bewegen sich sonst in 
einem Bereich von 4-5 \%. Für die Validitätskriterien Halbjahresnote Mathematik, BRC wird keine Kriteriumsvarianz aufgeklärt.

Tabelle 14: Darstellung der korrigierten Varianzaufklärung $\left(\mathrm{r}^{2}{ }_{\mathrm{KORR}}\right)$ für sieben Validitätskriterien.

\begin{tabular}{l|c}
\hline Validitätskriterien & $\begin{array}{l}\text { Anteil der aufgeklärten Varianz an der } \\
\text { Gesamtvarianz } \text { r }^{2} \text { KORR }\end{array}$ \\
\hline Lehrereinschätzung der Eignung & .04 \\
Halbjahresnote Englisch & .05 \\
Leistungszufriedenheit der Schüler & .04 \\
Halbjahresdurchschnittsnote aller Fächer & .05 \\
Halbjahresnote Deutsch & .13 \\
Halbjahresnote Mathematik & .00 \\
Halbjahresnote BRC & .00 \\
\hline
\end{tabular}

Anmerkung. BRC bezeichnet ein Wirtschaftsfach mit den Bereichen Betriebswirtschaft, Rechnungswesen und Controlling.

\subsubsection{Güte der Leistungstests}

\section{Reliabilitäten und Retestreliabilitäten}

Im Rahmen der zweiten Datenerhebung wurden ebenfalls Daten zu den Leistungstests erhoben, welche als Grundlage für die weitere Berechnung der Güte der Leistungstests dienten. Aufbauend auf den Ergebnissen der ersten Studie wurden keine weiteren TestSchwierigkeiten errechnet.

Anhand dieser Daten sollten die Reliabilitäten (interne Konsistenz) der relevanten Bausteine der Leistungstests bestimmt, um einen Vergleich mit den berechneten Reliabilitäten der ersten Studie zu ermöglichen (siehe Tabelle 15). So wurden für den Gesamtwert des Leistungstests Englisch sowie für den Untertest Deutsch: Wortschatz bezüglich der Höhe der internen Konsistenz ähnliche Werte erzielt. Die Reliabilität des Gesamttests Deutsch verschlechtert sich ein wenig im Vergleich zur ersten Studie. Auch für den Leistungstest im Fach Mathematik kann man einen leichten Abfall der Reliabilitäten des Gesamttestwertes sowie des Untertests: Funktion beobachten. Es ist trotzdem zu betonen, dass in den Daten eine relative Stabilität der berechneten internen Konsistenzen erkennbar ist, die nur in einem kleinen Bereich schwanken. 
Tabelle 15: Reliabilitäten der Leistungstests der Fächer Deutsch, Englisch und Mathematik (über beide Testversionen).

\begin{tabular}{|l|c|l|r|l|r|}
\hline \multicolumn{2}{|c|}{ Leistungstest Englisch } & \multicolumn{2}{c|}{ Leistungstest Deutsch } & \multicolumn{2}{c|}{ Leistungstest Mathematik } \\
\hline Gesamt & $\alpha=.77$ & Gesamt & $\alpha=.32$ & Gesamt & $\alpha=.59$ \\
\hline Übersetzen & $\alpha=.79$ & Wortschatz & $\alpha=.80$ & Funktion & $\alpha=.62$ \\
\hline
\end{tabular}

Anmerkung. Die diesen Berechnungen zugrunde liegende Stichprobengröße liegt zwischen $\mathrm{N}=87$ und $\mathrm{N}=89$ Schülern.

Im dieser zweiten Studie wurden zusätzlich zu den im vorigen Abschnitt dargestellten Reliabilitäten auch Retestreliabilitäten berechnet. Der 1. Testzeitpunkt dieser Retest-Erhebung entspricht dem 1. Messzeitpunkt der vorliegenden Studie (März 2005). Der zweite Testzeitpunkt zur Erhebung der Leistungstests ist kurz nach der Einschulung der aufgenommenen Schüler im August 2005 anzusiedeln. Aus organisatorischen Gründen war es nicht möglich, eine gezielte Zuordnung der Schüler zu den gleichen Testversionen des 1. Messzeitpunktes vorzunehmen, so dass die beiden Testformen per Zufall gleichmäßig auf alle Schüler verteilt wurden. Dieses Vorgehen führte zu der relativ geringen Stichprobengröße. Die Retestreliabilität wurde ebenfalls für alle hinsichtlich der Vorhersagegleichungen relevanten Bausteine der Leistungstests berechnet (siehe Tabelle 16).

Tabelle 16: Retestreliabilitäten der Leistungstests der Fächer Deutsch, Englisch, Mathematik (über beide Testversionen).

\begin{tabular}{|l|l|l|l|l|l|}
\hline \multicolumn{2}{|c|}{ Leistungstest Englisch } & \multicolumn{2}{c|}{ Leistungstest Deutsch } & \multicolumn{2}{c|}{ Leistungstest Mathematik } \\
\hline Gesamt & $\mathrm{r}_{\mathrm{tt}}=.65^{* *}$ & Gesamt & $\mathrm{r}_{\mathrm{tt}}=.44^{* *}$ & Gesamt & $\mathrm{r}_{\mathrm{tt}}=.64^{* *}$ \\
\hline Übersetzen & $\mathrm{r}_{\mathrm{tt}}=.68^{* *}$ & Wortschatz & $\mathrm{r}_{\mathrm{tt}}=.48^{* *}$ & Funktion & $\mathrm{r}_{\mathrm{tt}}=.54^{* *}$ \\
\hline
\end{tabular}

Anmerkung. Die diesen Berechnungen zugrunde liegende Stichprobengröße liegt für den Leistungstest Englisch bei $\mathrm{N}=50$, für den Leistungstest Deutsch bei $\mathrm{N}=44$ und für den Leistungstest Mathematik bei $\mathrm{N}=41$ Schülern.

Hinsichtlich der berechneten Retestreliabilitäten werden größtenteils zufrieden stellende Werte erzielt. Lediglich für den Gesamttest Deutsch erreicht dieser Wert eine wenig zufrieden stellende Höhe, die allerdings mit der Höhe der internen Konsistenz dieses Tests vergleichbar ist. Die Retestreliabilität für den Untertest Deutsch: Wortschatz verschlechtert sich deutlich im Vergleich zu dem Wert für die interne Konsistenz. In diesem Zusammenhang ist einerseits auf die geringe Stichprobengröße und zum anderen auf das für eine Retestung sehr große Zeitintervall von vier Monaten hinzuweisen. Es stellt sich hier die Frage, inwieweit dieses relativ große Zeitintervall zu einer fehlerbehafteten Schätzung der Retestreliabilität beitrug. Aus diesem Grunde kann man davon ausgehen, dass die hier berechneten Werte unterschätzt 
wurden und bei günstigeren Rahmenbedingungen höhere Werte erzielt werden könnten. Diese Argumentation ist insbesondere für den Untertest Deutsch: Wortschatz von Relevanz, da hier ein deutlich von den übrigen Reliabilitätswerten zur internen Konsistenz abweichender Wert berechnet wurde. Bei optimaleren Messbedingungen könnte dieser Wert sicherlich eine zufrieden stellende Höhe erreichen.

Insgesamt kann der Schluss gezogen werden, dass fast alle in das Diagnoseinstrumentarium eingehenden Prädiktorvariablen ausreichende Reliabilitäten aufweisen. Einen kritischen Wert weist lediglich der Gesamtwert für den Leistungstest Deutsch auf, welcher hinsichtlich der internen Konsistenz sowie der Retestreliabilität eine geringe Höhe erzielt. Bereits unter Abschnitt 4.3.3 der ersten Studie zur Güte der Leistungstests wurde damit argumentiert, dass dies sicherlich auch darauf zurückzuführen ist, dass dieser Test inhaltlich und strukturell sehr verschiedene Bestandteile in sich vereint und dass bei der Konstruktion dieses Tests darauf geachtet wurde, dieses Fach möglichst gut im Sinne einer hohen inhaltlichen Validität abzubilden. Einbußen der Reliabilität sind aus diesem Blickwinkel durchaus verständlich. Trotzdem handelt es sich hier um einen Leistungstest, der hinsichtlich seiner Gesamtzuverlässigkeit noch weiter optimiert und verbessert werden sollte. Zusammenfassend kann festgehalten werden, dass die in das Diagnoseinstrumentarium eingehenden relevanten Bestandteile der Leistungstests weitgehend die geforderte Zuverlässigkeit aufweisen.

\subsubsection{Fazit und weiteres Vorgehen zur Optimierung der Vorhersage- gleichungen}

Die Analysen zur zweiten Studie beinhalten einen Vergleich der Güte der Auswahl des herkömmlichen notenbasierten mit dem neuen testbasierten Verfahren. Hierzu wurde die im Rahmen der Schülerauswahl entstandene Gruppeneinteilung zugrunde gelegt (ausgewählt aufgrund: (1) ehemaligem Verfahren, (2) neuem Verfahren, (3) beiden Verfahren. Die Analysen bezogen sich größtenteils auf die für die Auswahlentscheidung relevanten Validitätskriterien Eignungsurteil der Lehrer und durchschnittliche Halbjahresnote. In diesem Zusammenhang wurden zunächst Vergleiche der Gruppenmittelwerte der Schülergruppen 1,2 und 3 für die beiden Kriterien durchgeführt. Weitere Analysen bezogen sich auf die Sensitivität (Analysen zur Trefferquote) sowie die Sensitivität und Spezifität (Analysen mittels ROC-Kurven) der Auswahl beider Verfahren im Vergleich. Anhand der Ergebnisse in diesen Analysen wird der Vorteil des neuen testbasierten Verfahrens deutlich. Dieser ist 
hinsichtlich der Kriterien Eignungsurteil in den meisten Fällen statistisch bedeutsam, bezüglich des Kriteriums durchschnittliche Halbjahresnote kann dieser Vorsprung statistisch nicht abgesichert werden.

Die Analyse der Korrelationen der beiden Rangreihen mit den Validitätskriterien stellt eine wertvolle Ergänzung dieser Analysen dar und bezieht weitere Validitätskriterien, wie die Endjahresnoten und die psychometrischen Leistungstests der Fächer Mathematik und Englisch ein. Anhand dieser Ergebnisse wird der Vorteil des testbasierten Auswahlverfahrens insbesondere aufgrund der psychometrischen Leistungstests deutlich, in welchen nach dem neuen Verfahren ausgewählte Schüler signifikant besser abschneiden. Hinsichtlich der Validitätskriterien Noten halten sich beide Verfahren ungefähr die Waage.

Aufgrund dieser Ergebnisse kann von der Gültigkeit der Hypothese zur prognostischen Überlegenheit des entwickelten Diagnoseinstrumentariums ausgegangen werden. Der signifikante Vorteil des neuen Verfahrens ist insbesondere anhand der besseren Ergebnisse für das Validitätskriterium Eignungsurteil sowie des hohen Zusammenhangs mit den psychometrischen Leistungstests begründbar. Es handelt sich hierbei um Validitätskriterien, die aufgrund ihrer Inhaltsvalidität und Güte am deutlichsten die Eignung der Schüler für die Oberstufe des Wirtschaftsgymnasiums abbilden. Anhand der Ergebnisse zum Vergleich der drei Schülergruppen für das Kriterium Eignungsurteil der Lehrer wird deutlich, dass eine geringe Redundanz der von beiden Verfahren erfassten Informationen besteht. Diese Befunde würden eine Kombination beider Verfahren bei der Schülerauswahl befürworten.

Für das weitere Vorgehen wurden die an der ersten Stichprobe erstellten Vorhersagegleichungen aufgrund der neu erhobenen Daten an der zweiten Kohorte für die Validitätskriterien Eignungsurteil der Lehrer und durchschnittliche Halbjahresnote weiter optimiert. Zusätzlich zu diesen vorliegenden Daten wurden die Datensätze beider Kohorten zu einer Gesamtstichprobe zusammengeführt. Auf der Basis dieser größeren Stichprobe wurden alternative Vorhersagegleichungen erstellt. Schließlich wurden für die ursprünglichen und alternativen Gleichungen Vorhersagen der Kriterien in allen drei Datensätzen (1. Stichprobe, 2. Stichprobe, Gesamtstichprobe) durchgeführt und die vorhergesagten Kriteriumswerte mit den erhobenen Kriteriumswerten korreliert. Aufgrund dieser Korrelationskoeffizienten sowie inhaltlicher Überlegungen zu relevanten Prädiktoren und deren Gewichtung wurden die Vorhersagegleichungen einem Vergleich unterzogen. Für beide Kriterien erwiesen sich die an der Gesamtstichprobe erstellten Vorhersagegleichungen als günstiger im Vergleich zu den 
ursprünglichen Vorhersagegleichungen. Die Gleichungen sind jeweils hinsichtlich der einbezogenen Prädiktoren den vorherigen Gleichungen aus der ersten Stichprobe sehr ähnlich. Die Gewichtungen wurden geringfügig verändert, die Reihenfolge der Stärke der Gewichtung veränderte sich kaum.

Im endgültigen Diagnoseinstrumentarium wurden die für die beiden optimierten Vorhersagegleichungen der Kriterien Eignungsurteil der Lehrer und Halbjahresdurchschnittsnote relevanten Prädiktoren beibehalten, alle anderen Prädiktorvariablen wurden entfernt. Die Leistungstests Deutsch, Mathematik und Englisch wurden beibehalten, wobei hieraus die Untertests Deutsch: Wortschatz, Mathematik: Funktion und Englisch: Übersetzen sowie ein Gesamtleistungswert (Durchschnitt der erzielten prozentualen Endergebnisse der Leistungstests) als Prädiktorvariablen in die Vorhersagegleichungen eingehen. Zum Bereich psychologischer Verfahren wurde der N-Test 2 (Figurenanalogien aus dem Kognitiven Fähigkeitstest) sowie die Skalen Selbstkonzept Deutsch (aus dem Verfahren zur Erfassung des differentiellen Selbstkonzepts schulischer Leistungen und Fähigkeiten, DISK-Gitter) und Elaborationsstrategien (aus dem Pisa-Fragebogen zum Selbstregulierten Lernen) im Instrumentarium belassen.

Es resultiert ein knappes, ökonomisches Verfahren, welches sich auf die Erhebung der relevantesten Prädiktorvariablen beschränkt. Auch hinsichtlich der Berechnung der Rangreihe wurde das Vorgehen weitestgehend vereinfacht und automatisiert. Die Daten werden in eine speziell dafür vorbereitete Excel-Datei eingegeben und auf Grundlage dieser Daten wird anhand eines festen Algorithmus, welcher die Vorhersagegleichungen beinhaltet, automatisch die Rangreihenberechnung vorgenommen. 


\section{Gesamtdiskussion}

In diesem Kapitel sollen die Ergebnisse beider Studien vor dem Hintergrund des globalen Projektziels der Verbesserung der Schülerauswahl zusammenfassend betrachtet werden. Zunächst wird ein Überblick über die beiden durchgeführten Teilstudien sowie deren zentrale Ergebnisse gegeben. In diesem Zusammenhang werden weitere grundlegende Fragen diskutiert.

Das vorrangige Projektziel beinhaltete die Konzipierung eines neuen Verfahrens, welches zuverlässiger als das bisherige System die Auswahl qualifizierter Schülern am Wirtschaftsgymnasium ermöglicht. Konkret ging es in diesem Projekt darum, dass mittels des neuen Vorgehens insbesondere diejenigen Schülerinnen und Schüler ausgewählt werden sollten, die besonders geeignet sind, die gymnasiale Oberstufe erfolgreich zu durchlaufen und zu beenden. Im Zuge dessen sollte langfristig die Quote der Schulabbrecher gesenkt und die Zahl der erfolgreichen Schulabgänger erhöht werden.

Die Zusammenarbeit bestand in der gemeinsamen Konstruktion, Optimierung und der Evaluation eines Diagnoseinstrumentariums. Hierfür wurden Leistungstests der Kernfächer Deutsch, Mathematik und Englisch entwickelt und optimiert. Des Weiteren wurden relevante Bausteine zur Erfassung individueller psychischer Merkmale zusammengestellt. Dies betraf die Bereiche Konzentration, formallogisches Denken, Leistungsmotivation, Selbstkonzept, Selbstwirksamkeitserwartung und Selbstreguliertes Lernen. Die Konzeption des Instrumentariums wurde so angelegt, dass ein zunächst großer „Pool“ von relevanten Bausteinen anhand empirischer Befunde immer weiter optimiert und auf seine wichtigsten Bestandteile konzentriert werden konnte. Strukturell sind zwei große Teilprozesse erkennbar: 1) in einer ersten Studie wurden die Bestandteile des Diagnoseinstrumentariums erstmalig erprobt und ein Prozedere für die Schülerauswahl entwickelt, 2) in einer zweiten Studie wurde eine weitere Validierung und Optimierung des Instrumentariums vorgenommen sowie der Frage nach der durch das neue Instrumentarium im Vergleich zum ehemaligen Auswahlverfahren erzielten inkrementellen Validität nachgegangen. Als zentrale Analysemethode wurden multiple Regressionsanalysen durchgeführt. Die Analysestrategie beruhte auf der Vorhersage von Validitätskriterien durch früher erfasste Prädiktorvariablen des entwickelten Diagnoseinstrumentariums. 
Im Rahmen der ersten Studie wurde untersucht, inwieweit es mittels der erhobenen Prädiktorvariablen gelingt, verschiedene Validitätskriterien aufzuklären. Hier interessierte zum einen die Höhe der erzielten Varianzaufklärung als Hinweis auf die Prädiktor- sowie Kriteriengüte, zum anderen die Frage, welche der Prädiktorvariablen zur Varianzaufklärung beitrugen und wie daraufhin eine erste Optimierung des Instrumentariums aussehen soll. In diesem Zusammenhang war auch von Interesse, inwieweit die zusätzlich zum Bereich Vorwissen erhobenen Prädiktorvariablen der Bereiche kognitive Grundfertigkeiten, Motivation und Selbstregulation weitere inkrementelle Varianz aufklärten. Darüber hinaus wurde davon ausgegangen, dass das Diagnoseinstrumentarium im Vergleich zum herkömmlichen Verfahren zu einer Erhöhung der prognostischen Validität beiträgt. Mittels der erhobenen Prädiktorvariablen gelang es, maximal $49 \%$ der Kriteriumsvarianz aufzuklären. Die erzielten korrigierten Varianzaufklärungen bewegen sich im Bereich zwischen $10 \%$ und $49 \%$, wobei nur das Validitätskriterium Halbjahresnote im Fach BRC (Betriebswirtschaft, Rechnungswesen, Controlling) einen geringen Wert von ungefähr $10 \%$ erreicht. Mit diesen Werten übertrifft das neue testbasierte Instrumentarium die durch das herkömmliche notenbasierte Verfahren erzielte Varianzaufklärung im Durchschnitt um das Sechs- bis Siebenfache. In diese Analysen gingen Prädiktorvariablen aus fast allen einbezogenen Determinantenbereichen ein. Einen großen Stellenwert nimmt hier - wie in Kapitel 2 zum theoretischen und empirischen Hintergrund bereits vermutet - das Vorwissen mit den Leistungstests der Fächer Mathematik, Deutsch und Englisch ein. Ebenfalls relevant für die Vorhersage mehrerer Kriterien erwiesen sich Prädiktorvariablen der Bereiche kognitive Grundfertigkeiten, Motivation und Selbstregulation. So konnte anhand der Daten der ersten Studie gezeigt werden, dass der Einbezug psychologischer Verfahren eine durchschnittliche Verbesserung der durch das Vorwissen erzielten Varianzaufklärung um das Drei- bis Vierfache erbrachte. Diese erste Studie diente unter anderem dazu, relevante und aussagekräftige Variablen für das Diagnoseinstrumentarium herauszufiltern. Aufgrund der durchgeführten Analysen konnte das Instrumentarium auf seine relevantesten Bestandteile gekürzt werden.

In diesem Zusammenhang stellt sich die Frage, wie die geringe prognostische Validität der Schulnoten, auf welchen das herkömmliche Verfahren basierte, zu erklären ist (dieses Verfahren beruhte hauptsächlich auf den Endjahresnoten der Fächer Deutsch, Mathematik und Englisch der Herkunftsschulen). Die Bedeutung bisheriger Schulleistungen für die Vorhersage zukünftigen Schulerfolges wird kontrovers diskutiert (vgl. Sauer, 2001). Ingenkamp (1995) kritisiert beispielsweise, dass Noten allein nur unzulänglich in der Lage 
sind, Schüler anhand ihrer Leistungsfähigkeit auszuwählen, weil sie in unzureichendem Maße objektiv, reliabel und valide sind. Andererseits werden Schulnoten häufig, gerade im Kontext des Wechsels in andere Zweige des Bildungssystems, systematisch zur Vorhersage von zukünftigem Schulerfolg eingesetzt und bewiesen in Längsschnittstudien eine relativ große Stabilität (vgl. z.B. Sauer \& Gamsjäger, 1996). Im vorliegenden Fall ist zu betonen, dass sich bei dem Übergang auf die Oberstufe des Wirtschaftsgymnasiums die Schultypen der abgebenden Schulen voneinander unterscheiden. Die geringe prognostische Validität der Zeugnisnoten, auf denen das herkömmliche Auswahlverfahren beruhte, könnte darin begründet liegen, dass die Lehrer der Herkunftsschulen bei der Beurteilung der Schulleistung ihren jeweiligen klassen- und schulinternen Referenzrahmen heranziehen. Dem neuen Diagnoseinstrumentarium hingegen liegt ein für alle Schüler gleicher Bewertungsmaßstab zugrunde, welcher zu prognostisch valideren wie auch faireren Aussagen über zukünftigen Schulerfolg führt.

Im Rahmen der zweiten Studie ging es aufbauend auf den Ergebnissen der ersten Untersuchung hauptsächlich um die Überprüfung der prognostischen Validität des optimierten Diagnoseinstrumentariums im Vergleich zum herkömmlichen Auswahlverfahren. Hiefür wurden beide Verfahren zur Schülerauswahl eines 11. Jahrgangs eingesetzt. Vorhersagen über den zukünftigen Schulerfolg der Bewerber basierten für das neue Diagnoseinstrumentarium hauptsächlich auf den Validitätskriterien Eignungsurteil der Lehrer sowie durchschnittliche Halbjahresnote. Dieses Vorgehen ermöglichte die spätere Durchführung von Analysen zur Sensitivität und Spezifität der Auswahl beider Verfahren im Vergleich hinsichtlich dieser beiden Validitätskriterien. Zum anderen wurden Korrelationen zwischen erhobenen Validitätskriterien und den anhand beider Verfahren zur Auswahl erstellten Rangreihen berechnet.

Aufgrund der Ergebnisse der zweiten Studie kann davon ausgegangen werden, dass das neue Auswahlverfahren dem herkömmlichen Vorgehen überlegen ist. Der signifikante Vorteil des neuen Verfahrens zeigte sich unter anderem anhand der besseren, zum Teil statistisch signifikanten, Ergebnisse für das Validitätskriterium Eignungsurteil der Lehrer. Die erwarteten Effekte fielen für dieses Kriterium bedeutsamer aus als für das Kriterium durchschnittliche Halbjahresnote. Es stellt sich hier die Frage, wie die Güte und folglich auch die Relevanz der beiden Kriterien bei der Beurteilung dieser Ergebnisse bewertet werden kann.

Das Kriterium Eignungsurteil der Lehrer nimmt insofern eine besondere Stellung ein, da es nicht auf der Basis von Noten, sondern aufgrund subjektiver Einschätzungen von 
verschiedenen Fachlehrern vorgenommen wurde. In dieses Urteil gehen vermutlich stärker Ressourcen und Potentiale der Schüler ein als in die Halbjahresnoten, die hier nur den aktuellen Leistungsstand eines Schülers nach einem ersten halben Jahr an der Schule widerspiegeln. Für das Eignungsurteil wurde auch danach gefragt, inwieweit ein Lehrer die Wahrscheinlichkeit einschätzt, dass ein Schüler das Abitur an dieser Schule schafft. Es ist anzunehmen, dass ein solches Urteil über die Halbjahresnoten hinausgehend Entwicklungspotentiale sowie lern- und leistungsrelevante individuelle Merkmale der Schüler beinhaltet und somit das Idealbild, welches das Wirtschaftsgymnasium von einem geeigneten Schüler hat, stärker abbildet. Diese Vermutung spiegelt sich insofern in den Daten wider, dass das im Dezember 2005 erhobene Eignungsurteil tendenziell stärker mit der im Juli 2006 erfassten durchschnittlichen Endjahresnote (r=.68** (nach Pearson), N=99) als mit der im Januar 2006 erhobenen durchschnittlichen Halbjahresnote ( $\mathrm{r}=.61 * *$ (nach Pearson), $\mathrm{N}=106)$ korreliert. Für die Endjahresnoten im Vergleich zu den Halbjahresnoten ist anzunehmen, dass diese Bewertungen über einen größeren Zeitraum umfassen und dadurch eine bessere Einschätzung der Schüler ermöglichen. Hieraus könnte man ableiten, dass das Eignungsurteil, wie oben vermutet, ein umfassenderes Urteil über einen Schüler beinhaltet. Des Weiteren kann diese Vermutung auch mit Blick auf die zur Aufklärung dieses Kriteriums eingehenden Prädiktorvariablen untermauert werden. Hier gehen viel stärker als für die durchschnittliche Halbjahresnote Prädiktoren der Bereiche kognitive Grundfertigkeiten, Motivation und Selbstregulation ein. An dieser Stelle ist auch auf den erzielten höheren Wert der korrigierten Varianzaufklärung von $49 \%$ im Vergleich zu 38 \% für die durchschnittliche Halbjahresnote hinzuweisen, der als ein weiterer Hinweis auf die höhere Güte des Validitätskriteriums Eignungsurteil gesehen werden kann.

Vor dem Hintergrund des ursprünglichen Ziels des Wirtschaftsgymnasiums, möglichst langfristig gute Schülerinnen und Schüler auszuwählen, die den Anforderungen der Schule gerecht werden und das Abitur erfolgreich absolvieren, sollte ein besonderes Gewicht auf Ressourcen und Entwicklungsmöglichkeiten der Schüler gelegt werden. Insbesondere aufgrund des Schulwechsels kann es eine Weile dauern, bis sich manche Schüler auf die veränderten Anforderungen eingestellt haben und ihr optimales Leistungspotential ausschöpfen können. Diese Leistungspotentiale werden vermutlich von dem Kriterium Eignungsurteil stärker erfasst als durch die Halbjahresnoten. Bei der Beurteilung der Steigerung prognostischen Validität des neuen testbasierten Auswahlverfahrens ist es diesen Überlegungen folgend durchaus sinnvoll, dem Validitätskriterium Eignungsurteil ein stärkeres Gewicht zuzusprechen. 
In einer weiteren Analyse im Rahmen der zweiten Studie wurden Korrelationen zwischen verschiedenen Validitätskriterien und den durch beide Auswahlverfahren erstellten Rangreihen berechnet. Diese einbezogenen Validitätskriterien der zweiten Studie können in drei Gruppen eingeteilt werden: 1) das Eignungsurteil der Lehrer, die Einschätzung der eigenen Leistungszufriedenheit der Schüler sowie die Halbjahresnoten, 2) die Endjahresnoten sowie 3) die psychometrischen Leistungstests der Fächer Englisch und Mathematik. Der vermutete Vorteil des neuen Verfahrens gegenüber dem ehemaligen Auswahlvorgehen drückte sich hinsichtlich dieser Korrelationen eindeutig anhand der psychometrischen Leistungstests aus. Hier stellt sich die Frage, wie diese verschiedenen Validitätskriterien in Bezug auf ihre Relevanz bewertet werden sollten. Ein Bewertungsgesichtspunkt betrifft die zeitliche Nähe der Erhebung dieser zu den Prädiktorvariablen, wobei ein größerer Abstand noch eine bessere Abschätzung der prognostischen Validität der Kriterien ermöglicht. Während die Prädiktorvariablen im März 2005 erfasst wurden, wurde das Eignungsurteil sowie die Leistungszufriedenheit im Dezember 2005, die Halbjahresnoten Ende Januar 2006 erhoben. Diese drei Kriteriengruppen wurden ungefähr zum gleichen Zeitpunkt erfasst und beziehen sich auf den Zeitraum, der das erste Halbjahr der Schüler an der Schule betrifft. Die psychometrischen Leistungstests wiederum wurden im Februar 2006 durchgeführt und spiegeln den aktuellen Kenntnisstand der Schüler zu diesem Zeitpunkt wider. Die Endjahresnoten weisen den zeitlich größten Abstand zur Erhebung der Prädiktorvariablen auf, diese wurden im Juli 2006 vergeben. Sie repräsentieren sozusagen die Leistungen der Schülerinnen und Schüler über ein Schuljahr und beinhalten erwartungsgemäß die Halbjahresnoten. Die Endjahresnoten stellen sicherlich im Vergleich zu den Halbjahresnoten die günstigeren und valideren Kriterien dar, da sie Noten über zwei Halbjahre beinhalten und den Schülern somit eine längere Zeitspanne zur Verfügung stand, sich an die Anforderungen der neuen Schule und der Sekundarstufe II zu gewöhnen. Hier ist zusammenfassend zu sagen, dass die Validitätskriterien Eignungsurteil, Leistungszufriedenheit sowie Halbjahresnoten hinsichtlich des Erhebungszeitpunktes vergleichbar sind. Die zeitlich am weitesten entfernten Kriterien sind die Endjahresnoten sowie die psychometrischen Leistungstests.

Ein anderer Aspekt zur Beurteilung der Bedeutsamkeit der einzelnen Kriterien ist die inhaltliche Validität dieser Kriterien hinsichtlich des zugrunde liegenden Konstruktes Schulerfolg. Für das Kriterium Eignungsurteil der Lehrer wurde dies bereits an früherer Stelle dieses Kapitels getan. Aufgrund des Einbezugs von Prädiktoren der Bereiche kognitive Grundfertigkeiten, Motivation und Selbstregulation sowie der relativ hohen Varianzaufklärung im Rahmen der ersten Studie (49 \%) wird von einer guten inhaltlichen 
Validität des Kriteriums Eignungsurteil ausgegangen. Die als Validitätskriterien eingesetzten Noten bieten sicherlich ein gutes Abbild der erzielten konkreten Leistungen der Schüler. Hier ist allerdings zu bedenken, dass Noten mitunter mangelnde Objektivität, Reliabilität und Vergleichbarkeit vorgeworfen wird (vgl. Ingenkamp, 1995). Die psychometrischen Leistungstests sind bezogen auf ihre Gütemerkmale als hoch zu beurteilen. Es handelt sich hierbei um unabhängige, objektive und hinsichtlich weiterer psychometrischer Kennwerte erprobte Verfahren, welche einen fairen Vergleich der Schülerinnen und Schüler ermöglichen. Es ist davon auszugehen, dass von den Schülern hier verschiedenste Fertigkeiten sowie Transferleistungen verlangt wurden. Derartige psychometrische Leistungstests weisen einige Vorteile gegenüber Noten auf, beispielsweise besitzen diese in der Regel mehr Objektivität, eine höhere Reliabilität und eine bessere Vergleichbarkeit (vgl. Ingenkamp, 1995). Hinsichtlich dieser Kriterien erzielte das neu entwickelte Diagnoseinstrumentarium einen deutlich signifikanten Vorteil gegenüber dem herkömmlichen Verfahren. Dieser Vorteil ist nachvollziehbar, wenn man bedenkt, dass das Diagnoseinstrumentarium darauf beruht, dass die Schüler unter anderem Leistungstests sowie einen Test zum schlussfolgernden Denken bearbeiteten. Aufgrund dieser Tests werden vermutlich stärker die Schüler ausgewählt, die zum einen über fundiertes Wissen und Fähigkeiten in den Fächern Deutsch, Mathematik und Englisch verfügen sowie zum anderen dazu in der Lage sind, ihr Wissen und ihre Fähigkeiten auf andere und neue Aufgaben anzuwenden. Genau diese Fertigkeiten finden in einer erfolgreichen Durchführung der im Februar 2006 erhobenen psychometrischen Leistungstests Anwendung. Die psychometrischen Leistungstests überzeugen somit zum einen aufgrund des zeitlichen Abstandes ihrer Erhebung zu den Prädiktorvariablen sowie zum anderen durch ihre inhaltliche Validität.

An dieser Stelle ist es wichtig $\mathrm{zu}$ erwähnen, dass aufgrund methodischer Ähnlichkeiten zu den Noten ein Vorteil des herkömmlichen Verfahrens gegenüber dem neuen Verfahren hinsichtlich der Validitätskriterien Halb- und Endjahresnoten besteht. Da das herkömmliche Verfahren auf Endjahresnoten der Fächer Deutsch, Mathematik und Englisch beruht, weist es auch bezüglich der Aufklärung der Validitätskriterien in Form von Noten Vorteile gegenüber dem neuen Verfahren auf. Die tendenziell besseren Ergebnisse des herkömmlichen Verfahrens hinsichtlich der Korrelationen für die Endjahresnoten sind sicherlich aufgrund der beschriebenen methodischen Ähnlichkeit erklärbar. Aus diesem Grund wird im Vergleich beider Verfahren hinsichtlich dieser Kriterien die Prädiktionskraft des neuen testbasierten Verfahrens unterschätzt. Die Tatsache, dass das neue Verfahren bezüglich der Noten mindestens genauso gut auswählt wie das herkömmliche Verfahren 
spricht also auch für dessen Vorteil. Unterstützt wird diese Vermutung noch durch den deutlichen Vorsprung des Diagnoseinstrumentariums bezüglich der psychometrischen Leistungstests.

Aufgrund vorangehender Ausführungen wird eine hohe Güte der psychometrischen Leistungstests vorausgesetzt. Diese sind hinsichtlich ihrer Bedeutsamkeit als zu den Notenkriterien gleichrangig zu beurteilen. Die soeben dargestellte Argumentation spricht sogar noch für eine stärkere Gewichtung dieser Kriterien. Aufbauend auf diesen Überlegungen weist der signifikant höhere Zusammenhang des Diagnoseinstrumentariums hinsichtlich psychometrischer Leistungstests eindeutig auf den Vorteil des neuen Verfahrens hin. Die Hypothese zur prognostischen Überlegenheit des Diagnoseinstrumentariums hat sich somit bestätigt.

Die Ergebnisse der beiden Studien zeigen, dass die aufgestellten Hypothesen und Fragestellungen im Sinne der Erwartung beantwortet werden konnten. Sicherlich wäre in gewissem Maße noch eine Steigerung der Güte der Auswahl möglich. In diesem Zusammenhang sollen Überlegungen dazu angestellt werden, wie eine Optimierung des gewählten Vorgehens unter anderen Rahmenbedingungen aussehen könnte.

An dieser Stelle ist nochmals darauf hinzuweisen, dass dieses Projekt durch die Zusammenarbeit der Forschungsabteilung Pädagogische Psychologie und Entwicklungspsychologie mit einem Wirtschaftsgymnasium einen besonderen Stellenwert einnimmt. Eine solche Konstellation ermöglicht einerseits eine weitgehend direkte Übertragung aktueller Forschungsergebnisse in die Praxis. Auf der anderen Seite werden derartige Projekte oft von Rahmenbedingungen begleitet, die eine streng methodisch orientierte Forschung erschweren. Diese besonderen Rahmenbedingungen erklären einige der nachfolgend diskutierten Vorgehensweisen, machen aber auch den besonderen Reiz dieses Projektes aus. Derartige Rahmenbedingungen wurden bereits in Kapitel 2 im allgemeinen Prognosemodell von Krapp (1979) angesprochen. Es handelt sich hierbei um Rahmenbedingungen, die sich aus dem Handlungszusammenhang, also durch das spezielle vorgefundene Setting, ergeben. Hierzu gehört beispielsweise der festgelegte Zeitrahmen des Projektes oder die Art der für diesen Rahmen ausgewählten Prädiktorvariablen.

Die Güte des Diagnoseinstrumentariums bemisst sich anhand der Güte seiner einzelnen Bestandteile: (a) der Prädiktorvariablen, (b) der Validitätskriterien und (c) der Prognosegleichungen. 
Durch den ursprünglich breiten Pool von Prädiktorvariablen der verschiedensten Bereiche ist es gut gelungen ist, eine optimale Auswahl relevanter Variablen aufzustellen. So ist in der Endversion des Instrumentariums eine knappe Auswahl von Prädiktorvariablen verschiedener relevanter Bereiche vertreten, wobei dem Vorwissen hier ein besonderes Gewicht eingeräumt wurde. In einem relativ kurzen Zeitraum von maximal zwei Zeitstunden werden mit der endgültigen Version des Diagnoseinstrumentariums Leistungstests der Kernfächer erhoben, relevante individuelle Merkmale mittels Fragebogen erfasst sowie ein Test zum formallogischen Denken durchgeführt.

Zur Erfassung individueller Merkmale mittels Fragebogen gibt es berechtigte Einwände, die die Gefahr eines ungewollten Einflusses verschiedener Antworttendenzen in Fragebögen betreffen. Es wurde versucht, derartige Tendenzen $\mathrm{zu}$ vermeiden. So wurde beispielsweise einer Antworttendenz zur Mitte durch die Auswahl einer meist geraden Anzahl von Antwortmöglichkeiten entgegengewirkt. Eine weitere Tendenz stellen Antworten im Sinne sozialer Erwünschtheit dar. Aufgrund der guten empirischen Ergebnisse ist anzunehmen, dass diese Tendenz keine große Rolle spielt. Durch diese Antworttendenz wäre sicherlich am ehesten die in der Endversion des Instrumentariums verbliebene Skala Selbstkonzept Deutsch betroffen, weniger vermutlich die Skala zur Erfassung von Elaborationsstrategien. Der Fakt, dass in der Skala Elaborationsstrategien vermutlich etwas anderes erfasst wird, als ursprünglich angenommen, mag dazu beitragen, dass eine systematische Verfälschbarkeit dieser Skala schwieriger ist, beziehungsweise, dass für die Schüler schlecht einsehbar ist, welche Antworten erwünscht sein könnten.

Das Diagnoseinstrumentarium ist in seiner endgültigen Kombination der Prädiktorvariablen äußerst gelungen, da eine Mischung aus Leistungstests, ein Test zum formallogischen Denken (Untertest des Kognitiven Fähigkeitstests) sowie zwei Skalen zur Erfassung individueller Merkmale enthalten sind. Die Güte der aus den Leistungstests relevanten Bestandteile ist ebenfalls größtenteils im geforderten Maß zufrieden stellend. Im Rahmen der gegebenen Möglichkeiten beinhaltet das entwickelte Instrumentarium eine optimale Kombination verschiedener Variablen.

Die Güte des Diagnoseinstrumentariums wird ebenfalls durch die eingesetzten Validitätskriterien bestimmt. Es wurde darauf geachtet, eine möglichst gute Mischung verschiedener Kriterien zusammenzustellen. Die Kriterien spielen im Rahmen der ersten Studie eine besondere Rolle, da sie hier für die Entwicklung und erste Optimierung des Instrumentariums eingesetzt wurden. Die Güte dieser Validitätskriterien wurde in diesem Kapitel in einem anderen Zusammenhang bereits ausführlich diskutiert. Die selbst erstellten 
Skalen zur Erhebung des Eignungsurteils der Lehrer sowie der Einschätzung der Leistungszufriedenheit der Schüler weisen zufrieden stellende Reliabilitäten auf (Eignung: $\alpha=.90$; Leistungszufriedenheit: $\alpha=.73$ ). So wird davon ausgegangen, dass die erhobenen Kriterien den Schulerfolg im Sinne der inhaltlichen Validität gut abbilden. Die Wahl der beiden Kriterien Eignungsurteil und durchschnittliche Halbjahresnote als Grundlage für das weitere Vorgehen der Schülerauswahl mittels neuem Verfahren erweist sich auch im Rückblick anhand der Daten der zweiten Stichprobe als sinnvoll. So handelt es sich bei beiden Kriterien um weitgehend globale, übergreifende Urteile, die hinsichtlich der eingehenden Prädiktoren sowie der Ergebnisse der beiden Studien gute Ergebnisse erzielen. Trotzdem hätte die Entwicklung und erste Optimierung dieses Instrumentariums anhand psychometrischer Leistungstests in der ersten Studie eine gelungene Ergänzung der Validitätskriterien bedeutet. Derartige Datenerhebungen waren im ersten Teil des Projektes jedoch nicht möglich. Allerdings konnten zur Validierung und Beurteilung der Güte des optimierten Instrumentariums im Rahmen der zweiten Studie Daten aus psychometrischen Leistungstests als wertvolle Ergänzung zur Beurteilung der inkrementellen Validität des Diagnoseinstrumentariums im Vergleich zum ehemaligen Verfahren herangezogen werden.

Die Güte der Vorhersagegleichungen hängt ebenfalls stark mit der Frage der Stabilität der Vorhersagen bzw. der Übertragbarkeit der aufgestellten Gleichungen auf unterschiedliche Stichproben zusammen. Die Ergebnisse der zweiten Studie belegen, dass das neue Instrumentarium eine deutlich bessere Schülerauswahl ermöglicht. Das belegt eine gewisse Stabilität des an der ersten Stichprobe entwickelten und an einer anderen zweiten Stichprobe durchgeführten Vorgehens. Es fällt allerdings auf, dass die im Rahmen der zweiten Studie erzielten Werte zur Aufklärung der Kriteriumsvarianz im Vergleich zu den aus der ersten Studie vorliegenden Werten deutlich geringer ausfielen. Der höchste Wert erreicht eine Varianzaufklärung von $13 \%$ für die Halbjahresnote Deutsch. Alle anderen Werte bewegen sich in dem Bereich zwischen $0 \%$ und $5 \%$ korrigierter Varianzaufklärung. Die Werte der ersten Stichprobe bewegen sich für die meisten Kriterien im Bereich zwischen $24 \%$ und $49 \%$ erzielter Varianzaufklärung. Es stellt sich die Frage, wie es zu einem solchen deutlichen Verlust an Varianzaufklärung kommt. Ein sehr plausibler Erklärungsansatz stützt sich auf den Fakt, dass diese zweite Stichprobe bereits anhand des Diagnoseinstrumentariums ausgewählt wurde und somit vermutlich eine geringere Streuung hinsichtlich der hierfür vorliegenden Prädiktoren und Kriterien aufweist. Folglich müsste die erzielte Varianzaufklärung - aufgrund einer kleineren Varianz des Kriteriumsmaßes - eine geringere Höhe erreichen. Schaut man sich hierzu die relevanten Prädiktorvariablen der ersten und zweiten Stichprobe und deren 
Verteilung im Vergleich an, dann findet man Hinweise dafür, dass Varianzreduktion eingetreten ist. In der zweiten Stichprobe sind im Vergleich zur ersten Stichprobe größtenteils geringere Streuungen der Prädiktorvariablen zu finden. Diese Differenz erweist sich für die Prädiktorvariable Gesamtergebnis im Leistungstest Mathematik sogar als statistisch bedeutsam, was für die Angemessenheit dieses Erklärungsansatzes spricht.

Um die Stabilität der Vorhersage noch zu steigern wäre es für ähnliche Projekte sinnvoll, die Entwicklung und Optimierung eines solchen Verfahrens an mehr als zwei Stichproben vorzunehmen. Soweit es der zeitliche Rahmen ermöglicht, wäre es wünschenswert, wenn Vorhersagegleichungen über mehrere Stichproben kreuzvalidiert und optimiert würden. Dies könnte ein stabileres Vorgehen gewährleisten und die Vorhersagekraft weiterhin steigern. Im hier angelegten Rahmen wurden allerdings alle derartigen Möglichkeiten ausgeschöpft. Beispielsweise wurden die zunächst an der ersten Stichprobe entwickelten Vorhersagegleichungen weiterhin anhand der aus beiden Stichproben bestehenden Gesamtstichprobe optimiert.

Neben verschiedenen methodischen Argumenten zum Vorteil des neuen Verfahrens können auch noch andere relevante Punkte aufgeführt werden, die für den Einsatz des neuen Instrumentariums sprechen. Hier ist zum Beispiel die größere Fairness zu nennen, die den Schülern entgegengebracht wird, die sich für einen Platz an der Schule bewerben und an dem Auswahlverfahren teilnehmen. Diese Fairness liegt darin begründet, dass es sich um größtenteils reliable, valide und objektive Bestandteile des Instrumentariums und eine streng standardisierte und objektivierte Durchführung des Auswahlverfahrens handelt. Dieses Kriterium kann von dem ehemaligen Verfahren nicht eingehalten werden, da hierfür Noten verschiedener Schulen miteinander verglichen werden.

Für das weitere Vorgehen bei der Schülerauswahl ist zu überlegen, ob gegebenenfalls eine Kombination beider Verfahren dem alleinigen Einsatz des neuen Verfahrens vorgezogen werden sollte. So weisen einige der dargestellten Ergebnisse darauf hin, dass beide Auswahlverfahren offensichtlich wenig redundante Informationen enthalten. Dafür spricht auch die sehr geringe Korrelation (Spearmans rho) beider Rangreihen der im Endeffekt ausgewählten Schüler ( $r=.05, N=88$ (2. Stichprobe)). Für das weitere Vorgehen ist also eine Kombination beider Verfahren empfehlenswert, vorzugsweise nach einem ähnlichen Vorgehen, wie es bei der Auswahl im Rahmen der zweiten Studie getan wurde. Da das ehemalige Verfahren einen unerheblichen Mehraufwand an Zeit erfordert und bereits in 
langer Erprobung automatisiert wurde, ist dieser Vorschlag sicherlich nicht nur sinnvoll, sondern auch praktikabel.

Insgesamt kann resümiert werden, dass im Rahmen dieses Projektes ein funktionstüchtiges, reliables und valides Diagnoseinstrumentarium entwickelt wurde, welches eine prognostisch validere Vorhersage von Schulerfolg im Rahmen der Schülerauswahl ermöglicht. 


\section{Zusammenfassung}

Die Notwendigkeit einer gezielten und grundlegenden Verbesserung bestehender nationaler Bildungsangebote und Bildungsstrukturen ist spätestens seit der Veröffentlichung der Ergebnisse international vergleichender Schulleistungsstudien deutlich geworden. Die im Handlungskatalog der Kultusministerkonferenz (vgl. Kultusministerkonferenz, 2002) ausgearbeiteten relevanten Punkte bieten dabei eine Orientierung für das weitere Vorgehen bei der Optimierung der Bildungsqualität an deutschen Schulen.

Das durchgeführte Projekt setzt hinsichtlich seiner Zielstellung, der Verbesserung der Schülerauswahl an der Oberstufe eines Wirtschaftsgymnasiums, am Handlungsfeld „Qualitätssicherung von Bildung durch verbindliche Standards und ergebnisorientierte Evaluation“ des genannten Kataloges an. Ausgehend von dieser Fragestellung wurde zunächst eine Rahmenkonzeption für ein Auswahlverfahren zur Diagnose und Prognose von Schulleistung entwickelt. Dieses Auswahlverfahren besteht aus an Bildungsstandards orientierten Leistungstests der Fächer Mathematik, Deutsch und Englisch sowie aus relevanten Verfahren zur Erfassung individueller Merkmale der Bereiche kognitive Grundfertigkeiten, Motivation und Selbstregulation.

In einer ersten Studie wurde das Diagnoseinstrumentarium erstmalig an einem 11. Jahrgang des Wirtschaftsgymnasiums eingesetzt und hinsichtlich der Bedeutsamkeit der enthaltenen Prädiktorvariablen evaluiert. Eine daraufhin erfolgte Optimierung des Instrumentariums war Ausgangspunkt der zweiten Studie, in welcher das Diagnoseinstrumentarium gemeinsam mit dem herkömmlichen notenbasierten Verfahren der Schule zur Auswahl eines weiteren 11. Jahrgangs eingesetzt wurde.

Im Rahmen der ersten Studie wurden Fragen zur Relevanz und Bedeutsamkeit der im Diagnoseinstrumentarium enthaltenen Prädiktorvariablen sowie Fragen zur inkrementellen Validität der zusätzlich zu den Leistungstests eingesetzten ,psychologischen“ Verfahren der Bereiche kognitive Grundfertigkeiten, Motivation und Selbstregulation geklärt. In der zweiten Studie lag der Fokus vor allem auf Analysen zur vermuteten erhöhten Prognosesicherheit des neu entwickelten testbasierten Auswahlverfahrens bei der Schülerauswahl im Vergleich zum herkömmlichen notenbasierten Vorgehen des Wirtschaftsgymnasiums.

Zur Beantwortung der aufgestellten Hypothesen und Fragestellungen wurde mittels multipler Regressionsanalysen die Vorhersagekraft der im Diagnoseinstrumentarium enthaltenen Prädiktorvariablen auf zeitlich später erhobene Validitätskriterien des 
Schulerfolges geprüft. Die beiden Studien beinhalteten zwei Messzeitpunkte: die Erhebung der relevanten Prädiktorvariablen sowie die zeitlich nachfolgende Erhebung von Validitätskriterien zur Messung von Schulerfolg.

Die Daten der ersten Studie sprechen für eine deutliche Überlegenheit des Diagnoseinstrumentariums gegenüber dem herkömmlichen Auswahlverfahren der Schule hinsichtlich der Vorhersage der Validitätskriterien. Die zusätzliche Aufnahme der „psychologischen“ Verfahren zu den eingesetzten Leistungstests trug zu einer Verbesserung der bereits durch die Leistungstests erzielten Varianzaufklärung um das Drei- bis Vierfache bei. In der zweiten Studie konnte der Vorteil des Diagnoseinstrumentariums auch im Rahmen der Schülerauswahl bestätigt werden. Dieser Vorsprung wurde insbesondere bezogen auf die Ergebnisse psychometrischer Leistungstests deutlich, in denen von dem neuen Verfahren ausgewählte Schülerinnen und Schüler signifikant besser abschnitten.

Insgesamt zeigt sich, dass mit dem resultierenden Diagnoseinstrumentarium, beruhend auf Leistungstests sowie relevanten Variablen zur Erfassung individueller Merkmale, eine bessere Auswahlgüte erzielt werden kann als mit dem herkömmlichen Vorgehen. Die Ergebnisse sprechen dafür, dass das grundlegende Projektziel, die Verbesserung der Schülerauswahl an der Oberstufe des Wirtschaftsgymnasiums, erreicht wurde.

Weitere Vorteile des neuen Diagnoseinstrumentariums sind dessen Objektivität und größere Fairness, die starke Orientierung an den Bildungsstandards der Fächer Deutsch, Mathematik und Englisch sowie der Einbezug individueller Ressourcen der Schüler in deren Beurteilung.

Es ist wünschenswert, dass - trotz der Herausforderung, die eine Implementierung wissenschaftlicher Erkenntnisse in den schulischen Alltag bedeutet - weiterhin vergleichbare Projekte initiiert werden. 


\section{Literaturverzeichnis}

Artelt, C. (1999). Lernstrategien und Lernerfolg - Eine handlungsnahe Studie. Zeitschrift für Entwicklungspsychologie und Pädagogische Psychologie, 31, 86-96.

Artelt, C. (2000). Strategisches Lernen. Münster: Waxmann.

Artelt, C., Demmrich, A. \& Baumert, J. (2001). Selbstreguliertes Lernen. In Deutsches PisaKonsortium (Hrsg.), PISA 2000. Basiskompetenzen von Schülerinnen und Schülern im internationalen Vergleich (S. 271-298). Opladen: Leske+Budrich.

Backhaus, K., Erichson, B., Plinke, W. \& Weiber, R. (2000). Multivariate Analysemethoden. Eine anwendungsorientierte Einführung (9., überarbeitete und erweiterte Auflage). Berlin: Springer.

Bandura, A. (1997). Self Efficacy. The Exercise of Control. New York: Freeman.

Baumert, J., Bos, W. \& Lehmann, R. H. (Hrsg.) (2000). TIMSS/III. Dritte Internationale Mathematik- und Naturwissenschaftsstudie - Mathematische und naturwissenschaftliche Bildung am Ende der Schullaufbahn. Band I: Mathematische und naturwissenschaftliche Grundbildung am Ende der Pflichtschulzeit (S. 31 - 84). Opladen: Leske+Budrich.

Baumert, J., Klieme, E., Neubrand, M., Prenzel, M., Schiefele, U., Schneider, W., Tillmann, K.-J. \& Weiß, M. (Hrsg.). (2000). Fähigkeit zum selbstregulierten Lernen als fächerübergreifende Kompetenz. Berlin: Max-Planck-Institut für Bildungsforschung.

Berg, D. \& Imhof, M. (2001). Aufmerksamkeit und Konzentration. In D. H. Rost (Hrsg.), Handwörterbuch Pädagogische Psychologie (2. Aufl.) (S. 42-49). Weinheim: Beltz PVU.

Bjorklund, D. F. \& Harnishfeger, K. K. (1990). Children's strategies: Their definition and origins. In D. F. Bjorklund (Hrsg.), Children's strategies: Contemporary views of cognitive development (S. 309-323). Hillsdale: Erlbaum.

Boekaerts, M. (1994). Action control: How relevant is it for classroom learning? In J. Kuhl \& J. Beckmann (Eds.), Volition and Personality (pp. 427-435). Seattle: Hogrefe \& Huber. 
Boerner, S., Seeber, G., Keller, H. \& Beinhorn, P. (2005). Lernstrategien und Lernerfolg im Studium: Zur Validierung des LIST bei berufstätigen Studierenden. Zeitschrift für Entwicklungspsychologie und Pädagogische Psychologie, 37, 17-26.

Bortz, J. (1993). Statistik. Für Sozialwissenschaftler. (4. Aufl.) Berlin: Springer.

Brickenkamp, R. (2002). Test d2: Aufmerksamkeits-Belastungs-Test (9., überarbeitete und neu normierte Aufl.). Göttingen: Hogrefe.

Burns, R. B. (1979). The self-concept. Theory, measurement, development and behavior. New York: Longman.

Cavanaugh, J. C. (1989). The importance of awareness in memory aging. In L. W. Poon, D. C. Rubin \& B. A. Wilson (Eds.), Everyday cognition in adulthood and late life (pp. 416-436). Cambridge: Cambridge University Press.

Chi, M. T. H., Feltovich, P. J. \& Glaser, R. (1981). Categorization and representation of physics problems by experts and novices. Cognitive Science, 5, 121-152.

Corno, L. \& Kanfer, R. (1993). The role of volition in learning and performance. In L. Darling-Hammond (Ed.), Review of research in education (Vol. 19, pp. 3-43). Washington, DC: American Educational Research Association.

Deci, E. L. \& Ryan, R. M. (1993). Die Selbstbestimmungstheorie der Motivation und ihre Bedeutung für die Pädagogik. Zeitschrift für Pädagogik, 39. Jhg, S. 223-238.

Dickhäuser, O., Schöne, C., Spinath, B. \& Stiensmeier-Pelster, J. (2002). Die Skalen zum akademischen Selbstkonzept. Konstruktion und Überprüfung eines neuen Instrumentes, Zeitschrift für Differentielle und Diagnostische Psychologie, 23 (4), 393-405.

Dweck, C. S. (1986). Motivational processes affecting learning. American Psychologist, 41, 1040-1048.

Eccles, J. S. \& Wigfield, A. (1995). In the mind of the achiever: The structure of adolescents' academic achievement related beliefs and self-perceptions. Personality and Social Psychology Bulletin, 21, 215-225.

Ericsson, K. A. \& Crutcher, R. J. (1990). The nature of exeptional performance. In P. B. Baltes, D. L. Featherman \& R. M. Lerner (Eds.), Life-span development and behavior (Vol. 10, pp. 187-217). Hillsdale, NJ: Erlbaum. 
Fend, H. (1971). Konformität und Selbstbestimmung. Weinheim: Beltz.

Filipp, S.-H. \& Frey, D. (1987). Das Selbst. In Deutsches Institut für Fernstudien (Hrsg.), Funkkolleg Psychobiologie, Studienbegleitbrief 8 (S. 11-56). Weinheim: Beltz.

Flavell, J. H. (1976). Metacognitive aspects of problem solving. In L. B. Resnick (Ed.), The nature of intelligence (pp. 231-235). Hillsdale, NJ: Erlbaum.

Friedrich, H. F. (1995). Analyse und Förderung kognitiver Lernstrategien. Empirische Pädagogik, 9 (2), 115-153.

Glaser, R. (1991). The maturing of the relationship between the science of learning and cognition and educational practice. Learning and Instruction, 1, 129-144.

Hansford, B. C. \& Hattie, J. A. (1982). The relationship between self and achievement / performance measures. Review of Educational Research, 52, 123-142.

Hasselhorn, M. (1992). Metakognition und Lernen. In G. Nold (Hrsg.), Lernbedingungen und Lernstrategien. Welche Rolle spielen kognitive Verstehensstrukturen? (S. 35-63). Tübingen: Narr.

Hasselhorn, M. (2006). Metakognition. In D. H. Rost (Hrsg.), Handwörterbuch Pädagogische Psychologie (3. Aufl.) (S. 480-485). Weinheim: Beltz PVU

Heckhausen, H. (1972). Die Interaktion von Sozialisationsvariablen in der Genese des Leistungsmotivs. In C. F. Graumann (Hrsg.), Handbuch der Psychologie, B. 7/2, Sozialpsychologie (S. 955-1019). Göttingen: Hogrefe.

Heckhausen, H. (1989). Motivation und Handeln (2. Aufl.). Berlin: Springer.

Heller, K. (1997). Individuelle Faktoren der Schulleistung. Literaturüberblick. In F. E. Weinert \& A. Helmke (Hrsg.), Entwicklung im Grundschulalter (S. 183-201). Weinheim: Beltz PVU.

Heller, K. A. \& Hany, E. A. (2001). Standardisierte Schulleistungsmessungen. In F. E. Weinert (Hrsg.), Leistungsmessung in Schulen (S. 87-101). Weinheim: Beltz.

Heller, K. A. \& Perleth, C. (2000). Kognitiver Fähigkeitstest für 4. bis 12. Klassen, Revision (KFT 4-12+R). Göttingen: Hogrefe. 
Heller, K. A., Rosemann, B. \& Steffens, K.-H. (1978). Prognose des Schulerfolgs. Eine Längsschnittstudie zur Schullaufbahnberatung. Weinheim: Beltz.

Hellwig, H.-J. (1975). Zur Differenzierung von Intelligenztest- und Konzentrationsleistungen. Unveröffentlichte Dissertation, Technische Universität, Berlin.

Helmke, A. (1992a). Determinanten der Schulleistung: Forschungsstand und Forschungsdefizit. In G. Nold (Hrsg.), Lernbedingungen und Lernstrategien. Welche Rolle spielen kognitive Verstehensstrukturen? (S. 23-34). Tübingen: Narr.

Helmke, A. (1992b). Selbstvertrauen und schulische Leistungen. Göttingen: Hogrefe.

Helmke, A. \& Schrader, F.-W. (2001). Determinanten der Schulleistung. In D. H. Rost (Hrsg.), Handwörterbuch Pädagogische Psychologie (2. Aufl.) (S. 81-91). Weinheim: Beltz PVU.

Helmke, A. \& Weinert, F. E. (1997). Bedingungsfaktoren schulischer Leistungen. In F. E. Weinert (Hrsg.), Psychologie des Unterrichts und der Schule (S. 71-176). Göttingen: Hogrefe.

Holling, H., Preckel, F. \& Vock, M. (2004). Intelligenzdiagnostik. Göttingen: Hogrefe.

Horn, J. L. (1968). Organization of abilities and the development of intelligence. Psychological Review, 75, 242-259.

Ingenkamp, K. (Hrsg.) (1995). Die Fragwürdigkeit der Zensurengebung: Text und Untersuchungsberichte. Weinheim: Beltz.

Jerusalem, M. \& Mittag, W. (1999). Selbstwirksamkeit, Bezugsnormen, Leistung und Wohlbefinden in der Schule. In M. Jerusalem \& R. Pekrun (Hrsg.), Emotion, Motivation und Leistung (S. 223-245). Göttingen: Hogrefe.

Jerusalem, M. \& Satow, L. (1999). Skala zur schulbezogenen Selbstwirksamkeitserwartung (WIRKSCHUL). In R. Schwarzer \& M. Jerusalem (Hrsg.), Skalen zur Erfassung von Lehrer- und Schülermerkmalen (S. 18-19). Berlin: Freie Universität Berlin.

Keller, G. (1993). Das Lern- und Arbeitsverhalten leistungsstarker und leistungsschwacher Schüler. Psychologie in Erziehung und Unterricht, 40, 125-129.

Klauer, K. J. (2001). Intelligenz und Begabung. In D. H. Rost (Hrsg.), Handwörterbuch Pädagogische Psychologie (2. Aufl.) (S. 280-285). Weinheim: Beltz PVU. 
Köller, O. \& Möller, J. (2006). Selbstwirksamkeit. In D. H. Rost (Hrsg.), Handwörterbuch Pädagogische Psychologie (3. Aufl.) (S. 693-698). Weinheim: Beltz PVU.

Köller, O. \& Schiefele, U. (2001). Zielorientierung. In D. H. Rost (Hrsg.), Handwörterbuch Pädagogische Psychologie (S. 811-815). Weinheim: Beltz PVU.

Köller, O., Watermann, R., Trautwein, U. \& Lüdtke O. (2004). Wege zur Hochschulreife in Baden-Württemberg. TOSCA - Eine Untersuchung an allgemein bildenden und beruflichen Gymnasien. Opladen: Leske + Budrich.

Kraft, S. (1999). Selbstgesteuertes Lernen. Problembereiche in Theorie und Praxis. Zeitschrift für Pädagogik, 45, 833-845.

Krapp, A. (1973). Bedingungen des Schulerfolgs. München: R. Oldenbourg Verlag.

Krapp, A. (1979). Prognose und Entscheidung: zur theoretischen Begründung und Differenzierung der pädagogisch-psychologischen Prognose. Weinheim: Beltz.

Krapp, A. (1992a). Das Interessenkonstrukt. Bestimmungsmerkmale der Interessenhandlung und des individuellen Interesses aus der Sicht der Person-Gegenstands-Konzeption. In A. Krapp \& M. Prenzel (Hrsg.), Interesse, Lernen, Leistung. Neuere Ansätze der pädagogisch-psychologischen Interessenforschung. Münster: Aschendorff.

Krapp, A. (1992b). Interesse, Lernen und Leistung. Neue Forschungsansätze in der Pädagogischen Psychologie. Zeitschrift für Pädagogik, 38, 747-770.

Krapp, A. (1997). Selbstkonzept und Leistung - Dynamik ihres Zusammenspiels: Literaturüberblick. In F. E. Weinert \& A. Helmke (Hrsg.), Entwicklung im Grundschulalter (S. 325-339). Weinheim: Beltz PVU.

Krapp, A. (1998). Entwicklung und Förderung von Interessen im Unterricht. Psychologie in Erziehung und Unterricht, 44, 185-201.

Krapp, A. \& Mandl, H. (1977). Einschulungsdiagnostik. Weinheim: Beltz.

Kuhl, J. (1983). Motivation, Konflikt und Handlungskontrolle. Berlin: Springer.

Kuhl, J. (1998). Wille und Persönlichkeit: Funktionsanalyse der Selbststeuerung. Psychologische Rundschau, 49 (2), 61-77. 
Kühn, R. (1983). Bedingungen für Schulerfolg. Zusammenhänge zwischen Schülermerkmalen, häuslicher Umwelt und Schulnoten. In B. Kraak (Hrsg.), Studien zur Pädagogischen Psychologie, Band 18. Göttingen: Hogrefe.

Kultusministerkonferenz (2002), PISA 2000 - Zentrale Handlungsfelder: Zusammenfassende Darstellung der laufenden und geplanten Maßnahmen in den Ländern, Beschluss der 299. Kultusministerkonferenz vom 17./18.10.2002, Bonn.

Lukesch, H. (1998). Einführung in die pädagogisch-psychologische Diagnostik. Psychologie in der Lehrerausbildung, Band 3. Regensburg: Roderer.

Mack, W. (1995). Intelligenz und Expertiseerwerb. Empirische Studien zur Kompetenzentwicklung bei Kfz-Mechanikern. Dissertation. München: Ludwig-MaximiliansUniversität.

Marsh, H. W. (1990). The structure of academic self-concept: The Marsh-Shavelson model. Journal of Educational Psychology, 82, 623-636.

Marsh, H. W. \& Shavelson, R. J. (1985). Self-concept: Its multifaceted, hierarchical structure. Educational Psychologist, 20, 107-125.

Mittag, W., Kleine, D. \& Jerusalem, M. (2002). Evaluation der schulbezogenen Selbstwirksamkeit von Sekundarschülern. Zeitschrift für Pädagogik, 44. Beiheft, S. 145-173.

Moschner, B. (2001). Selbstkonzept. In D. H. Rost (Hrsg.), Handwörterbuch Pädagogische Psychologie (2. Aufl.) (S. 629-635). Weinheim: Beltz PVU.

Moschner, B. \& Dickhäuser, O. (2006). Selbstkonzept. In D. H. Rost (Hrsg.), Handwörterbuch Pädagogische Psychologie (3. Aufl.) (S. 685-692). Weinheim: Beltz PVU.

Nicholls, J. G. (1989). The competitive ethos and democratic education. Cambridge, MA: Harvard University Press.

Oswald, W.-D., Roth, E. (1978). Der Zahlen-Verbindungs-Test (ZVT). Göttingen: Hogrefe.

Pekrun, R. \& Schiefele, U. (1996). Emotions- und motivationspsychologische Bedingung der Lernleistung. In F. E. Weinert (Hrsg.), Psychologie des Lernens und der Instruktion (S.153-180). Göttingen: Hogrefe.

Preiser, S. (1998). Kontrollüberzeugungen. In: D. H. Rost (Hrsg.). Handwörterbuch Pädagogische Psychologie. Weinheim: Beltz. 
Renkl, A. (1996). Vorwissen und Schulleistung. In J. Möller \& O. Köller (Hrsg.), Emotionen, Kognitionen und Schulleistung. Weinheim: Beltz PVU.

Rheinberg, F. \& Wendland, M. (2003). Itemübersicht zum Fragebogen SSI-K-32. Potsdam: Universität Potsdam, Institut für Psychologie.

Rost, D. H. \& Lamsfuß, S. (1992). Entwicklung und Erprobung einer ökonomischen Skala zur Erfassung des Selbstkonzepts schulischer Leistungen und Fähigkeiten (SKSLF). Zeitschrift für Pädagogische Psychologie, 6, 239-250.

Rost, D. H. \& Sparfeldt, J. (2002). Facetten des schulischen Selbstkonzepts. Ein Verfahren zur Messung des differentiellen Selbstkonzepts schulischer Leistungen und Fähigkeiten (DISK-Gitter). Diagnostica, 48 (3), 130-140.

Roth, E. \& Sauer, J. (1981). Über die Entwicklung einiger kognitiver Bedingungen der Schulleistung. In K. Voppa \& R. Groner (Hrsg.), Kognitive Strukturen und ihre Entwicklung (S. 135-153). Bern: Huber.

Satow, L. (1999). Klassenklima und Selbstwirksamkeitsentwicklung. Eine Längsschnittstudie in der Sekundarstufe I. Unveröffentliche Dissertation. Berlin: Freie Universtität.

Satow, L. \& Schwarzer, R. (2000). Selbstwirksamkeitserwartung, Besorgtheit und Schulleistung. Eine Längsschnittuntersuchung in der Sekundarstufe I. Empirische Pädagogik, 14, 131-150.

Sauer, J. (2001). Prognose von Schulerfolg. In D. H. Rost (Hrsg.), Handwörterbuch Pädagogische Psychologie (2. Aufl.) (S. 544-555). Weinheim: Beltz PVU.

Sauer, J. \& Gamsjäger, E. (1996). Ist Schulerfolg vorhersagbar? Die Determinanten der Grundschulleistung und ihr prognostischer Wert für den Schulerfolg. Göttingen: Hogrefe.

Sauer, J. \& Gattringer, H. (1986). Zur Aufklärung der Schulleistung durch spezifische und gemeinsame Varianzanteile von Intelligenz und Motivation. In K. Daumenlang \& J. Sauer (Hrsg.), Aspekte psychologischer Forschung (S. 237-257). Göttingen: Hogrefe.

Schermer, J. S. (2001). Soziales Lernen. In: D. H. Rost (Hrsg.), Handwörterbuch Pädagogische Psychologie. Weinheim: Beltz PVU.

Schiefele, U. (1996). Motivation und Lernen mit Texten. Göttingen: Hogrefe. 
Schiefele, U., Krapp, A. \& Schreyer, I. (1993). Metaanalyse des Zusammenhangs von Interesse und schulischer Leistung. Zeitschrift für Entwicklungspsychologie und Pädagogische Psychologie, 25, 120-148.

Schiefele, U. \& Pekrun, R. (1996). Psychologische Modelle des fremdgesteuerten und selbstgesteuerten Lernens. In F. E. Weinert (Hrsg.), Psychologie des Lernens und der Instruktion (S.249-278). Göttingen: Hogrefe.

Schiefele, U. \& Urhahne, D. (2000). Motivationale und volitionale Bedingungen der Studienleistung. In U. Schiefele \& K.-P. Wild, Interesse und Lernmotivation. Untersuchungen zur Entwicklung, Förderung und Wirkung. Münster: Waxmann Verlag.

Schneider, W. (1985). Developmental trends in the metamemory-memory behavior relationship: An integrative review. In D. L. Forrest-Pressley, G. E. MacKinnon \& T. G. Waller (Eds.), Metacognition, cognition and human performance (Vol. 1, pp. 57-109). Orlando, FL: Academic Press.

Schneider, W. \& Bös, K. (1985). Exploratorische Analysen zu Komponenten des Schulerfolgs. Zeitschrift für Entwicklungspsychologie und Pädagogische Psychologie, 15, 325-340.

Schöne, C., Dickhäuser, O., Spinath, B. \& Stiensmeier-Pelster, J. (2003). Das Fähigkeitsselbstkonzept und seine Erfassung. In J. Stiensmeier-Pelster \& F. Rheinberg (Hrsg.), Diagnostik von Motivation und Selbstkonzept. Tests und Trends, Band 2 (S. 3-14). Göttingen: Hogrefe.

Schuler, H. \& Prochaska, M. (2001). LMI - Leistungsmotivationsinventar. Dimensionen berufsbezogener Leistungsorientierung. Göttingen: Hogrefe.

Schunk, D. H. \& Zimmerman, B. J. (Hrsg.). (1998). Self-regulated learning. From teaching to self-reflective practice. New York: Guilford Publications.

Schwarzer, R. \& Jerusalem, M. (2002). Das Konzept der Selbstwirksamkeit. Zeitschrift für Pädagogik. 44. Beiheft.

Seiffge-Krenke, I. (1987). Eine aktualisierte deutschsprachige Form des OFFER Self-Image Questionnaire. Zeitschrift für Differentielle und Diagnostische Psychologie, 8, 99-109.

Shavelson, R. J., Hubner, J. J. \& Stanton, G. C. (1976). Validation of construct interpretations. Review of Educational Research, 46, 407-441. 
Simon, H. A. (1990). Invariants of human behavior. Annual Review of Psychology, 41, 1-19.

Spada, H. \& Wichmann, S. (1996). Kognitive Determinanten der Lernleistung. In F. E. Weinert (Hrsg.), Psychologie des Lernens und der Instruktion (S.119-152). Göttingen: Hogrefe.

Spinath, B., Stiensmeier-Pelster, J., Schöne, C. \& Dickhäuser, O. (2002). Skalen zur Erfassung der Lern- und Leistungsmotivation (SELLMO). Göttingen: Hogrefe.

Stevenson, H. W., Parker, T., Wilkinson, A., Hegion, A. \& Fish, E. (1976). Longitudial study of individual differences in cognitive development and scholastic achievement. Journal of educational psychology, 68 (4), 377-400.

Stiensmeier-Pelster, J., Balke, S. \& Schlangen, B. (1996). Lern- versus Leistungszielorientierung als Bedingungen des Lernfortschritts. Zeitschrift für Entwicklungspsychologie und Pädagogische Psychologie, Band XXVIII, Heft 2, 169-187.

ten Hoopen, G. (1996). Auditive Aufmerksamkeit. In O. Neumann \& A. F. Sanders (Hrsg.), Aufmerksamkeit (S. 115-161). Göttingen: Hogrefe.

Utman, C. H. (1997). Performance effects of motivational state: A meta-analysis. Personality and Social Psychology Review, 1, 170-182.

Volet, S. E. (1997). Cognitive and effective variables in academic learning: The significance of direction and effort in students' goals. Learning and Instruction, 7, 235-254.

Weinert, F. E. (1982). Selbstgesteuertes Lernen als Voraussetzung, Methode und Ziel des Unterrichts. Unterrichtswissenschaft, 10, 99-110.

Weinert, F. E. (1984). Metakognition und Motivation als Determinanten der Lerneffektivität: einführung und Überblick. In F. E. Weinert \& R. H. Kluwe (Hrsg.), Metakognition, Motivation und Lernen (S. 9-21). Stuttgart: Kohlhammer.

Weinert, F. E. (1989). The impact of schooling on cognitive development: One hypothetical assumption, some empirical results, and many theoretical implications. Early News, 8, 37.

Weinert, F. E. (1996). Lerntheorien und Instruktionsmodelle. In F. E. Weinert (Hrsg.), Psychologie des Lernens und der Instruktion (S.1-48). Göttingen: Hogrefe. 
Weinert, F. E. \& Stefanek, J. (1997). Entwicklung vor, während und nach der Grundschulzeit. Ergebnisse aus dem SCHOLASTIK-Projekt. In F. E. Weinert, A. Helmke (Hrsg.), Entwicklung im Grundschulalter (S. 423-453). Weinheim: Beltz PVU.

Wigfield, A., Eccles, J. S. \& Rodriguez, D. (1998). The development of children's motivation in school contexts. In P. D. Pearson \& A. Iran-Nejad (Eds.), Review of research in education (Vol. 23, 73-118). Washington DC: American Educational Research Association. 


\section{Anhang}

\section{Anhangsverzeichnis}

Anhang A: Teststatistische Gütekriterien der Fragebögen, Subskalen und Tests der

Bereiche kognitive Grundfertigkeiten, Motivation und Selbstregulation 123

Anhang B: Lehrerfragebogen zur Einschätzung der Eignung der Schüler

Anhang C: Schülerfragebogen zur Einschätzung der eigenen schulischen

Leistungszufriedenheit

Anhang D: Instruktion für die Datenerfassung mittels Fragebogen - Studie 1

Anhang E: Instruktion für die Datenerfassung mittels Fragebogen - Studie 2 127

Anhang F: Fragebogen 1 - Studie 1 .............................................................................128

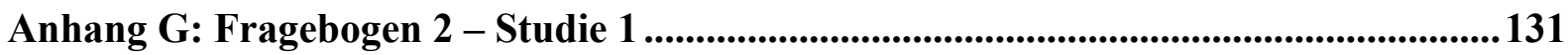

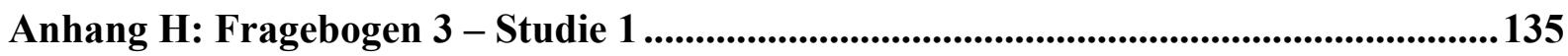

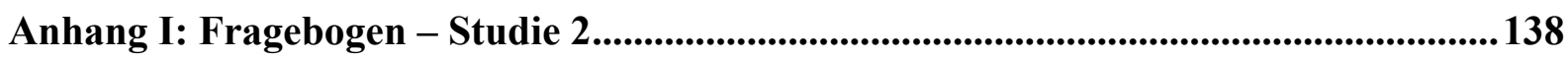

Anhang J: Skalen aus dem Pisa-Fragebogen zum Selbstregulierten Lernen, Baumert et

al. (2000)

Anhang K: Skala WIRSCHUL zur schulischen Selbstwirksamkeitserwartung,

Jerusalem \& Satow (1999) 145

Anhang L: DISK-Gitter, Rost \& Sparfeldt (2002) 146

Anhang M: Leistungsmotivationsinventar (Kurzform), Schuler \& Prochaska (2001)

Anhang N: Die Skalen Lernziele (N1) und Arbeitsvermeidung (N2) aus dem SELLMO,

Spinath et al. (2002).

Anhang O: Selbststeuerungsinventar-Kurzform (SSI-K-32), Rheinberg \& Wendland

Anhang P: Bedeutsame Korrelationen der Skala Elaborationsstrategien (Pisa-

Fragebogen) mit anderen Skalen

Anhang Q: Vorhersagegleichungen für die relevantesten Kriterien „Eignungsurteil der

Lehrer" sowie „durchschnittliche Halbjahresnote“ 152

Anhang R: Prädiktorvariablen und die dazugehörigen konreten Prädiktorwerte des

Diagnoseinstrumentariums 153 


\section{Anhang A: Teststatistische Gütekriterien der Fragebögen, Subskalen und Tests der Bereiche kognitive Grundfertig- keiten, Motivation und Selbstregulation}

Tabelle 17: Reliabilitäten (interne Konsistenz) und Trennschärfe der Skalen und Tests des erstellten Diagnoseinstrumentariums

\begin{tabular}{|c|c|c|c|}
\hline Fragebogen/Skalen/Tests & $\begin{array}{l}\text { Reliabilität der } \\
\text { Originalversion } \\
\text { (Cronbachs } \alpha)\end{array}$ & $\begin{array}{l}\text { Eigene Relia- } \\
\text { bilitätsanalyse } \\
(\text { Cronbachs } \alpha)\end{array}$ & $\begin{array}{c}\text { Trennschärfe } \\
\left(\mathbf{r}_{\text {it }}\right)\end{array}$ \\
\hline 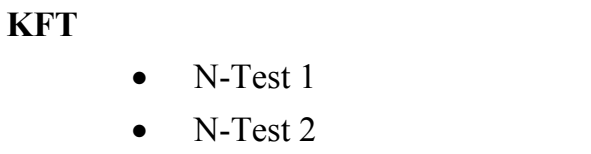 & $\begin{array}{c}\text { (Re- u. Parallel- } \\
\text { testung) } \\
.71-.93\end{array}$ & - & zufriedenstellend \\
\hline d2-Test & $\begin{array}{c}.94 \\
\text { (Konzentrations- } \\
\text { leistungswert) }\end{array}$ & - & - \\
\hline $\begin{array}{ll}\text { PISA } & \\
\text { - Wiederholungsstrategien } \\
\text { - Elaborationsstrategien } \\
\text { - Kontrollstrategien } \\
\text { - Selbstwirksamkeits- } \\
\text { - } \text { erwartungen } \\
\text { - Introntrollerwartungen } \\
\text { - Interesse Lesen } \\
\text { - Instrumentelle Motivation } \\
\text { - Handlungskontrolle: } \\
& \text { Anstrengung und Ausdauer }\end{array}$ & $\begin{array}{l}.74 \\
.75 \\
.72 \\
.75 \\
.72 \\
.76 \\
.85 \\
.83 \\
.77\end{array}$ & $\begin{array}{l}.85 \\
.77 \\
.64 \\
.75 \\
.72 \\
.89 \\
.82 \\
.84 \\
.80\end{array}$ & $\begin{array}{l}.46-.61 \\
.36-63 \\
.42-.54 \\
.49-.60 \\
.48-.52 \\
.47-.67 \\
.69-.77 \\
.68-.71 \\
.51-.62\end{array}$ \\
\hline WIRKSCHUL & $.70-.73$ & .77 & $.33 .-.51$ \\
\hline $\begin{aligned} \text { DISK-Gitter } \\
$\[ \]$ \\
\bullet \quad \text { Deutsch } \\
\bullet \quad \text { Englisch (angepasst) } \\
\bullet \quad \text { Mathematik }\end{aligned}$ & $\begin{array}{r}.93 \\
- \\
.95\end{array}$ & $\begin{array}{l}.91 \\
.95 \\
.96\end{array}$ & $\begin{array}{c}.66-.85 \\
- \\
.66-.90\end{array}$ \\
\hline LMI-K & .94 & .91 & $\begin{array}{l}\text { Daten nur für } \\
\text { Langversion } \\
\text { existent }\end{array}$ \\
\hline $\begin{aligned} \text { SELLMO } & \\
\bullet & \text { Lernziele } \\
\bullet & \text { Arbeitsvermeidung }\end{aligned}$ & $\begin{array}{l}.70-.79 \\
.77-.84\end{array}$ & $\begin{array}{l}.83 \\
.80\end{array}$ & $\begin{array}{l}.37-.51 \\
.42-.70\end{array}$ \\
\hline $\begin{aligned} \text { SSI-K-32 Gesamtwert } \\
\bullet \quad \text { Selbstkontrolle und } \\
\text { Zielverfolgung } \\
\bullet \quad \text { Energiemangel und geringe } \\
\text { Konzentration } \\
\text { - Selbstmotivierung und } \\
\text { Emotionskontrolle } \\
\text { - Tendenz zum Aufschieben }\end{aligned}$ & $\begin{array}{l}\text { Daten nur } \\
\text { für Langversion } \\
\text { vorhanden }\end{array}$ & $\begin{array}{l}.90 \\
.79 \\
.79 \\
.78 \\
.82\end{array}$ & $\begin{array}{c}\text { Daten nur } \\
\text { für Langversion } \\
\text { existent }\end{array}$ \\
\hline
\end{tabular}

Anmerkung. Die Werte der eigenen Reliabilitätsanalysen beziehen sich auf die

Daten der ersten Stichprobe 


\section{Anhang B: Lehrerfragebogen zur Einschätzung der Eignung der Schüler}

\section{Lehrerbefragung}

Im Folgenden bitten wir Sie darum, eine Einschätzung über den jeweiligen Schüler/ die jeweilige Schülerin (siehe Excel-Datei) abzugeben.

Vielen Dank für Ihre Mitarbeit.
(1)
(2)
(3)

1) Für wie geeignet halten Sie o.g. Schüler/ Schülerin für Ihre Schule?

2) Glauben Sie, dass o.g. Schüler/ Schülerin an Ihrer Schule erfolgreich sein wird?

3) Wie überzeugt sind Sie, dass o.g. Schüler/ Schülerin den Abschluss an Ihrer Schule schaffen wird?

4) Glauben Sie, dass o.g. Schüler/ Schülerin hier an der richtigen Schule ist? 


\section{Anhang C: Schülerfragebogen zur Einschätzung der eigenen schulischen Leistungszufriedenheit}

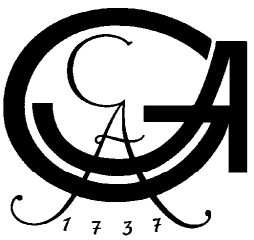

GEORG - AUGUST - UNIVERSITÄT

GEORG-ELIAS-MÜLLER-

INSTITUT

FÜR PSYCHOLOGIE

\section{Schülerbefragung}

Im Folgenden bitten wir Sie darum, Ihre persönliche Meinung abzugeben. Bitte lesen Sie dazu die folgenden Aussagen genau durch. Es gibt jeweils vier Antwortmöglichkeiten, mit deren Hilfe Sie angeben können, wie sehr ein Satz auf Sie zutrifft. Kreuzen Sie bitte das Kästchen an, welches am besten passt.

Vielen Dank für Ihre Mitarbeit.

Tragen Sie bitte hier Ihren persönlichen Code ein.

Code:

stimmt stimmt

gar eher stimmt stimmt

nicht nicht eher genau

1) Nach den ersten Monaten an der Arnoldi-Schule bin ich zuversichtlich, dass ich das Abitur schaffe.

2a) Ich fühle mich in meiner Klasse bezogen auf meine Kompetenzen an der richtigen Stelle.

2b) Ich fühle mich in meiner Klasse bezogen auf das Lernklima in der Klasse an der richtigen Stelle.

3a) Wenn ich sehe, was wir an der Arnoldi-Schule können müssen, halte ich mich für sehr geeignet.

3b) Wenn ich sehe, was wir an der Arnoldi-Schule lernen, entspricht das meinem beruflichen Interesse.

4) Mit den Anforderungen...

...im Fach BRC komme ich gut zurecht.

...im Fach Deutsch komme ich gut zurecht.

...im Fach Englisch komme ich gut zurecht.

...im Fach Mathematik komme ich gut zurecht.

5) Mit der Auswahl meines zukünftigen Leistungsfaches (Deutsch/Engl./Mathe) bin ich sehr zufrieden. 


\section{Anhang D: Instruktion für die Datenerfassung mittels Fragebogen - Studie 1}

Es sind im Folgenden 3 Fragebögen und noch 2 weitere Tests zu bearbeiten. Hiermit möchte ich Euch einige Hinweise zur Bearbeitung der Fragebögen geben.

1) Zunächst möchte ich darauf hinweisen, dass alle Angaben, die Ihr hier macht, vollständig anonym behandelt werden. Damit die einzelnen Fragebögen wieder ein und derselben Person zugeordnet werden können, hat jeder von Euch einen persönlichen Code, den er/sie auf jedem Fragebogen eintragen soll.

2) In den Fragebögen gibt es keine falschen und richtigen Antworten. Es soll keine Bewertung/Benotung der Antworten vorgenommen werden, sondern es geht lediglich um Eure persönliche Meinung. Bitte antwortet ehrlich!

3) In den einzelnen Fragebögen geht es darum zu beurteilen, inwieweit eine bestimmte Aussage auf Euch (Dich) persönlich zutrifft bzw. inwieweit Ihr (Du) einer bestimmten Aussage zustimmen könnt oder nicht. In den verschiedenen Fragebögen sind unterschiedlich viele Antwortmöglichkeiten vorgegeben. Es ist wichtig, dass Ihr jede Aussage bearbeitet. Fragebögen mit unbewerteten Aussagen können nicht ausgewertet werden.

Bitte setzt pro Aussage immer nur 1 Kreuz. Wenn Ihr zwischen zwei Antworten schwankt, dann versucht trotzdem Euch für die Antwort zu entscheiden, die am ehesten auf Euch zutrifft.

Markierungen zwischen zwei Zahlen können ebenfalls nicht ausgewertet werden.

4) Wenn Ihr versehentlich eine falsche Markierung gesetzt habt, so kreist diese bitte ein und setzt eine neue Markierung an der anderen Stelle.

5) Bevor Ihr anfangt ist es sehr wichtig, dass Ihr immer Euren persönlichen Code auf den Fragebogen eintragt!! Wenn dieser Code fehlt, dann kann der Fragebogen leider nicht verwendet werden.

6) Bearbeitet die Fragebögen bitte zügig, aber sorgfältig. Haltet Euch nicht allzu lange an einzelnen Aussagen auf. Wer mit dem jeweiligen Fragebogen fertig ist, der legt den Fragebogen sichtbar beiseite. 


\section{Anhang E: Instruktion für die Datenerfassung mittels Fragebogen - Studie 2}

„Nun ist noch ein Fragebogen auszufüllen. Hiermit möchte ich Euch einige Hinweise zur Bearbeitung des Fragebogens geben."

1) „Bitte lest jede Aussage genau durch und gebt an, inwieweit diese auf Euch persönlich zutrifft.

In dem Fragebogen gibt es keine falschen und richtigen Antworten, sondern es geht lediglich um Eure persönliche Meinung. Antwortet also bitte ehrlich!“‘

2) „In dem Fragebogen geht es darum zu beurteilen, inwieweit eine bestimmte Aussage auf Euch persönlich zutrifft bzw. inwieweit Ihr einer bestimmten Aussage zustimmen könnt oder nicht. Es sind unterschiedlich viele Antwortmöglichkeiten vorgegeben, mit deren Hilfe Ihr angeben könnt, wie sehr ein Satz auf Euch zutrifft. Trifft die Aussage für Euch gar nicht zu, dann setzt ein Kreuz im ersten Kästchen links. Trifft die Aussage vollständig auf Euch zu, dann setzt Euer Kreuz im Kästchen ganz rechts. Für verschiedene Abstufungen könnt Ihr die Kästchen dazwischen ankreuzen."

„Es ist wichtig, dass Ihr jede Aussage bearbeitet. Fragebögen mit unbewerteten Aussagen können nicht ausgewertet werden."

„Bitte setzt pro Aussage immer nur 1 Kreuz. Wenn Ihr zwischen zwei Antworten schwankt, dann versucht trotzdem Euch für die Antwort zu entscheiden, die am ehesten auf Euch zutrifft.“

„Markierungen zwischen zwei Zahlen bzw. zwei Kästchen können ebenfalls nicht ausgewertet werden."

„Wenn Ihr versehentlich eine falsche Markierung gesetzt habt, dann streicht diese bitte deutlich durch und setzt eine neue Markierung an einer anderen Stelle.“

3) „Bitte beachtet, dass sich die Anzahl der Abstufungen der Antwort innerhalb des Fragebogens verändert. Zunächst sind es sieben, dann sechs und am Ende nur noch fünf Abstufungen der Antwortmöglichkeiten.“

4) „Bearbeitet den Fragebogen bitte zügig, aber sorgfältig. Haltet Euch nicht allzu lange an einzelnen Aussagen auf. Wer mit dem jeweiligen Fragebogen fertig ist, der legt den Fragebogen sichtbar beiseite.“

5) „Bitte vergesst auch diesmal nicht, Euren Namen etc. einzutragen bevor Ihr mit dem Ausfüllen beginnt.“

„Noch Fragen?““

Beim Einsammeln bitte kontrollieren, ob der Name leserlich in den dafür vorgesehenen Kasten eingetragen wurde! 


\title{
Anhang F: Fragebogen 1 - Studie 1
}

\author{
(a) \\ GEORG-AUGUST-UNIVERSITÄT \\ GÖTTINGEN
}

\section{Hinweise zur Bearbeitung des Fragebogens}

Mit dem vorliegenden Fragebogen werden Ihre Einstellungen gegenüber Arbeit, Beruf und Leistung erfasst. Nur Ihre persönliche Meinung zählt - es gibt weder richtige noch falsche Antworten.

Bitte lesen Sie jede Aussage genau durch und geben Sie an, inwieweit sie auf Sie persönlich zutrifft. Sie können hierbei zwischen den Antwortmöglichkeiten (1) und (7) abstufen. Wenn eine Aussage gar nicht auf Sie persönlich zutrifft, markieren Sie die (1). Trifft die Aussage hingegen vollständig auf Sie zu, markieren Sie die (7). Zwischen (1) und (7) können Sie abstufen, je nachdem, in welchem Ausmaß die Aussage auf Sie zutrifft.

Bitte nehmen Sie Ihre Markierung stets eindeutig vor. Markierungen zwischen zwei Zahlen können nicht ausgewertet werden. Haben Sie versehentlich eine falsche Markierung vorgenommen, so kreisen Sie diese bitte ein und setzen Sie eine neue Markierung an einer anderen Stelle.

Wichtig ist, dass Sie jede Aussage bearbeiten. Fragebogen mit unbewerteten Aussagen können nicht ausgewertet werden. Wählen Sie im Zweifelsfall diejenige Antwortmöglichkeit, die noch am ehesten auf Sie zutrifft.

Bearbeiten Sie den Fragebogen bitte zügig, aber sorgfältig; halten Sie sich nicht zu lange an einzelnen Aussagen auf.

Tragen Sie bitte hier Ihren persönlichen Code ein.

\section{CODE:}




\section{trifft trifft \\ gar nicht zu vollständig zu}

1. Ich habe mir vorgenommen, es beruflich weit $\mathrm{zu}$ (1)-(2)-(3)-(4)-(5)-(6)-(7)

2. Ich bin überzeugt davon, dass ich es beruflich zu etwas bringen werde.

(1)-(2)-(3)-(4)-(5)-(6)-(7)

3. Mir sind Aufgaben lieber, die mir leicht von der Hand gehen, als solche, bei denen ich mich sehr einsetzen muss.

4. Manchmal lüge ich, wenn ich muss.

(1)-(2)-(3)-(4)-(5)-(6)-(7)

5. Ich bin überzeugt, mich bisher in Ausbildung und

Beruf mehr engagiert zu haben als meine Kollegen.

6. Ich empfinde Befriedigung darüber, meine eigene Leistung zu steigern.

(1)-(2)-(3)-(4)-(5)-(6)-(7)

7. Aufgaben, bei denen ich nicht ganz sicher bin, ob ich sie lösen kann, reizen mich ganz besonders.

(1)-(2)-(3)-(4)-(5)-(6)-(7)

8. Es ist mir sehr wichtig, eine verantwortungsvolle Position zu erreichen.

(1)-(2)-(3)-(4)-(5)-(6)-(7)

9. Ich nehme niemals Dinge an mich, die mir nicht gehören.

(1)-(2)-(3)-(4)-(5)-(6)-(7)

(1)-(2)-(3)-(4)-(5)-(6)-(7)

0. Wenn ich eine Prüfung ablege, bin ich auch davon überzeugt, dass ich sie bestehe.

(1)-(2)-(3)-(4)-(5)-(6)-(7)

1. Mein Ehrgeiz ist leicht herauszufordern.

(1)-(2)-(3)-(4)-(5)-(6)-(7)

12. Ich beschäftige mich besonders gern mit

Problemen, bei denen es eine harte Nuss zu knacken gibt.

13. Es ist schon einmal vorgekommen, dass ich jemanden ausgenutzt habe.

(1)-(2)-(3)-(4)-(5)-(6)-(7)

4. Ich bin zuversichtlich, dass meine Leistung die Anerkennung anderer finden wird.

(1)-(2)-(3)-(4)-(5)-(6)-(7)

5. Ich suche mir gern Aufgaben, an denen ich meine Fähigkeiten prüfen kann.

(1)-(2)-(3)-(4)-(5)-(6)-(7)

16. Am glücklichsten bin ich mit einer Aufgabe, bei der ich alle meine Kräfte einsetzen kann.

(1)-(2)-(3)-(4)-(5)-(6)-(7)

Ich bin schon einmal wegen einer angeblichen Krankheit nicht zur Arbeit oder Schule gegangen.

(1)-(2)-(3)-(4)-(5)-(6)-(7)

(1)-(2)-(3)-(4)-(5)-(6)-(7)

8. Wenn mir etwas nicht so gut gelungen ist, wie ich es mir vorgenommen hatte, strenge ich mich anschließend noch mehr an.

19. Der Wunsch, besser zu sein als andere, ist ein großer Ansporn für mich.

20. Ich fluche niemals.

$$
\text { (1)-(2)-(3)-(4)-(5)-(6)-(7) }
$$

21. Schwierige Probleme reizen mich mehr als einfache. 


\section{trifft trifft \\ gar nicht zu vollständig zu}

22. Auch wenn ich vor schwierigen Aufgaben stehe, bin ich immer guten Mutes.

(1)-(2)-(3)-(4)-(5)-(6)-(7)

23. Ich habe Dinge getan, von denen ich anderen nichts erzähle.

(1)-(2)-(3)-(4)-(5)-(6)-(7)

24. Wenn ich mit anderen zusammenarbeite, übernehme ich gewöhnlich die Initiative.

(1)-(2)-(3)-(4)-(5)-(6)-(7)

25. Meine Bekannten würden es als typisch für mich ansehen, dass ich mich durch alle Schwierigkeiten durchbeiße.

(1)-(2)-(3)-(4)-(5)-(6)-(7)

26. Ich empfinde Befriedigung über intensive, konzentrierte Arbeit.

27. Ich arbeite gern an Aufgaben, die ein hohes Maß an Geschick erfordern.

28. Manchmal zahle ich es lieber anderen heim, als dass ich vergebe und vergesse.

29. Es bereitet mir Freude, mich ganz in eine Aufgabe zu vertiefen.

(1)-(2)-(3)-(4)-(5)-(6)-(7)

0. Manchmal fahre ich schneller, als es erlaubt ist.

$$
\text { (1)-(2)-(3)-(4)-(5)-(6)-(7) }
$$

31. Es ist für mich ein beruflicher Ansporn, einmal eine wichtige Stellung zu erreichen.

$$
\text { (1)-(2)-(3)-(4)-(5)-(6)-(7) }
$$

Ich glaube, dass ich mich beruflich mehr anstrenge als die meisten meiner Kollegen.

(1)-(2)-(3)-(4)-(5)-(6)-(7)

Ich habe schon einmal zuviel Wechselgeld

herausbekommen, ohne es der Verkäuferin zu sagen.

(1)-(2)-(3)-(4)-(5)-(6)-(7)

(1)-(2) - (3)-(4)-(5)-(6)-(7)

34. Wenn ich mit anderen zusammenarbeite, nehme ich die Sachen gern selbst in die Hand.

(1)-(2)-(3)-(4)-(5)-(6)-(7)

35. Ich eigne mir lieber neue Kenntnisse an, als mich

mit Dingen zu beschäftigen, die ich schon beherrsche.

36. Wenn ich etwas erreicht habe, lag das vor allem an meinem Geschick und meinen Fähigkeiten.

$$
\text { (1)-(2)-(3)-(4)-(5)-(6)-(7) }
$$

$$
\text { (1)-(2)-(3)-(4)-(5)-(6)-(7) }
$$

7. Durch eine schwierige Aufgabe fühle ich mich besonders herausgefordert.

(1)-(2)-(3)-(4)-(5)-(6)-(7)

Ich gebe grundsätzlich alles an, was ich zu verzollen habe.

(1)-(2)-(3)-(4)-(5)-(6)-(7)

9. Wenn ein Risiko besteht, eine Aufgabe nicht zu schaffen, gebe ich mir ganz besondere Mühe.

(1)-(2)-(3)-(4)-(5)-(6)-(7)

(1)-(2)-(3)-(4)-(5)-(6)-(7)

0. Es ist mir wichtig, meine Tüchtigkeit zu steigern.

(1)-(2)-(3)-(4)-(5)-(6)-(7)

(1)-(2)-(3)-(4)-(5)-(6)-(7) 


\title{
Anhang G: Fragebogen 2 - Studie 1
}

\author{
C. GEORG-AUGUST-UNIVERSITÄT \\ GÖTTINGEN
}

\section{Hinweise zur Bearbeitung des Fragebogens}

Bitte lesen Sie jede Aussage genau durch und geben Sie an, inwieweit sie auf Sie persönlich zutrifft. Nur Ihre persönliche Meinung zählt - es gibt weder richtige noch falsche Antworten.

Es gibt jeweils sechs Antwortmöglichkeiten, mit deren Hilfe Sie angeben können, wie sehr ein Satz auf Sie zutrifft. Trifft die Aussage für Sie gar nicht zu, dann setzen Sie ein Kreuz im ersten Kästchen links. Trifft die Aussage für Sie vollständig zu, setzen Sie Ihr Kreuz im Kästchen ganz rechts. Für verschiedene Abstufungen kreuzen sie bitten die Kästchen dazwischen an.

Bitte nehmen Sie Ihre Markierung stets eindeutig vor. Markierungen zwischen zwei Zahlen können nicht ausgewertet werden. Haben Sie versehentlich eine falsche Markierung vorgenommen, so kreisen Sie diese bitte ein und setzen Sie eine neue Markierung an einer anderen Stelle.

Wichtig ist, dass Sie jede Aussage bearbeiten. Fragebogen mit unbewerteten Aussagen können nicht ausgewertet werden. Wählen Sie im Zweifelsfall diejenige Antwortmöglichkeit, die noch am ehesten auf Sie zutrifft.

Bearbeiten Sie den Fragebogen bitte zügig, aber sorgfältig; halten Sie sich nicht zu lange an einzelnen Aussagen auf.

Tragen Sie bitte hier Ihren persönlichen Code ein.

\section{CODE:}




\begin{tabular}{|c|c|c|c|c|c|c|}
\hline & $\begin{array}{l}\text { trifft } \\
\text { gar } n\end{array}$ & & & & $\begin{array}{l}\text { trifft } \\
\text { vollst }\end{array}$ & ig zu \\
\hline $\begin{array}{l}\text { 1. Wenn ich lerne, versuche ich alles auswendig zu } \\
\text { lernen, was drankommen könnte. }\end{array}$ & 0 & 0 & 0 & 0 & 0 & 0 \\
\hline $\begin{array}{l}\text { 2. Ich kann auch die schwierigen Aufgaben im } \\
\text { Unterricht lösen, wenn ich mich anstrenge. }\end{array}$ & 0 & 0 & 0 & 0 & 0 & 0 \\
\hline $\begin{array}{l}\text { 3. Ich lerne, um meine Berufschancen zu } \\
\text { verbessern. }\end{array}$ & 0 & 0 & 0 & 0 & 0 & 0 \\
\hline $\begin{array}{l}\text { 4. Wenn ich lerne, überlege ich, inwiefern die } \\
\text { Information im wirklichen Leben nützlich sein } \\
\text { könnte. }\end{array}$ & 0 & 0 & 0 & 0 & 0 & 0 \\
\hline $\begin{array}{l}\text { 5. Ich lerne, um eine gute Arbeitsstelle zu } \\
\text { bekommen. }\end{array}$ & 0 & 0 & 0 & 0 & 0 & $O$ \\
\hline $\begin{array}{l}\text { 6. Wenn ich lerne, passe ich genau auf, dass ich das } \\
\text { Wichtigste behalte. }\end{array}$ & 0 & 0 & 0 & 0 & 0 & 0 \\
\hline $\begin{array}{l}\text { 7. Es fällt mir leicht, neuen Unterrichtsstoff zu } \\
\text { verstehen. }\end{array}$ & 0 & 0 & $\mathrm{O}$ & 0 & 0 & O \\
\hline $\begin{array}{l}\text { 8. Wenn ich lerne, versuche ich, neuen Stoff mit } \\
\text { Dingen zu verbinden, die ich in anderen Fächern } \\
\text { gelernt habe. }\end{array}$ & 0 & 0 & 0 & 0 & 0 & 0 \\
\hline $\begin{array}{l}\text { 9. Weil mir das Lesen Spaß macht, würde ich es } \\
\text { nicht gerne aufgeben. }\end{array}$ & 0 & 0 & 0 & 0 & 0 & $\mathrm{O}$ \\
\hline $\begin{array}{l}\text { 10. Wenn ich lerne und etwas nicht verstehe, suche } \\
\text { ich nach zusätzlicher Information, um das } \\
\text { Problem zu klären. }\end{array}$ & 0 & 0 & 0 & 0 & 0 & 0 \\
\hline $\begin{array}{l}\text { 11. Wenn ich lerne, übe ich, indem ich den Stoff } \\
\text { immer wieder aufsage. }\end{array}$ & 0 & 0 & 0 & 0 & 0 & 0 \\
\hline $\begin{array}{l}\text { 12. Wenn ich lerne, versuche ich den Stoff besser zu } \\
\text { verstehen, indem ich Verbindungen zu Dingen } \\
\text { herstelle, die ich schon kenne. }\end{array}$ & 0 & 0 & 0 & 0 & 0 & 0 \\
\hline 13. Ich lese in meiner Freizeit. & $\mathrm{O}$ & O & $\mathrm{O}$ & $\mathrm{O}$ & $\mathrm{O}$ & $\mathrm{O}$ \\
\hline $\begin{array}{l}\text { 14. Wenn ich lerne, lerne ich so viel wie möglich } \\
\text { auswendig. }\end{array}$ & $\mathrm{O}$ & $\mathrm{O}$ & $\mathrm{O}$ & 0 & O & O \\
\hline $\begin{array}{l}\text { 15. Selbst wenn ich mal längere Zeit krank sein } \\
\text { sollte, kann ich immer noch gute Leistungen } \\
\text { erzielen. }\end{array}$ & $\mathrm{O}$ & $\mathrm{O}$ & $\mathrm{O}$ & O & $\mathrm{O}$ & $\mathrm{O}$ \\
\hline $\begin{array}{l}\text { 16. Wenn ich lerne, überlege ich, wie der Stoff mit } \\
\text { dem zusammenhängt, was ich schon gelernt } \\
\text { habe. }\end{array}$ & $\mathrm{O}$ & O & $\mathrm{O}$ & O & O & O \\
\hline $\begin{array}{l}\text { 17. Wenn ich lerne, versuche ich beim Lesen } \\
\text { herauszufinden, was ich noch nicht richtig } \\
\text { verstanden habe. }\end{array}$ & $\mathrm{O}$ & O & $\mathrm{O}$ & O & O & O \\
\hline $\begin{array}{l}\text { 18. Wenn ich mir vornehme, keine schlechten Noten } \\
\text { zu bekommen, dann gelingt es mir. }\end{array}$ & $\mathrm{O}$ & O & O & 0 & O & O \\
\hline
\end{tabular}




\begin{tabular}{|c|c|c|c|c|c|c|}
\hline & $\begin{array}{l}\text { trifft } \\
\text { gar } n\end{array}$ & & & & lliftä & $\lg \mathrm{zu}$ \\
\hline $\begin{array}{l}\text { 19. Ich bin überzeugt, dass ich in Hausaufgaben und } \\
\text { Klassenarbeiten gute Leistungen erzielen kann. }\end{array}$ & $\mathrm{O}$ & $\mathrm{O}$ & O & $\mathrm{O}$ & $\mathrm{O}$ & O \\
\hline $\begin{array}{l}\text { 20. Auch wenn der Lehrer/die Lehrerin an meinen } \\
\text { Fähigkeiten zweifelt, bin ich mir sicher, dass ich } \\
\text { gute Leistungen erzielen kann. }\end{array}$ & $\mathrm{O}$ & $\mathrm{O}$ & $\mathrm{O}$ & $\mathrm{O}$ & $\mathrm{O}$ & O \\
\hline 21. Wenn ich lerne, gebe ich mein Bestes. & $\mathrm{O}$ & $\mathrm{O}$ & O & $\mathrm{O}$ & O & O \\
\hline $\begin{array}{l}\text { 22. Wenn ich lerne, arbeite ich so fleißig wie } \\
\text { möglich. }\end{array}$ & O & O & O & O & O & O \\
\hline $\begin{array}{l}\text { 23. Wenn ich mir vornehme, bei keiner Aufgabe } \\
\text { einen Fehler zu machen, gelingt es mir. }\end{array}$ & $\mathrm{O}$ & $\mathrm{O}$ & $\mathrm{O}$ & $\mathrm{O}$ & $\mathrm{O}$ & O \\
\hline $\begin{array}{l}\text { 24. Wenn ich lerne, überlege ich mir zuerst, was } \\
\text { genau ich lernen muss. }\end{array}$ & $\mathrm{O}$ & $\mathrm{O}$ & $\mathrm{O}$ & $\mathrm{O}$ & O & O \\
\hline $\begin{array}{l}\text { 25. Wenn ich lerne, präge ich mir alles Neue so ein, } \\
\text { dass ich es aufsagen kann. }\end{array}$ & $\mathrm{O}$ & $\mathrm{O}$ & $\mathrm{O}$ & $\mathrm{O}$ & $\mathrm{O}$ & O \\
\hline $\begin{array}{l}\text { 26. Wenn ich lese, vergesse ich manchmal alles um } \\
\text { mich herum. }\end{array}$ & $\mathrm{O}$ & $\mathrm{O}$ & $\mathrm{O}$ & $\mathrm{O}$ & $\mathrm{O}$ & O \\
\hline $\begin{array}{l}\text { 27. Ich bin überzeugt, dass ich auch den } \\
\text { kompliziertesten Stoff, den der Lehrer vorstellt, } \\
\text { verstehen kann. }\end{array}$ & $\mathrm{O}$ & $\mathrm{O}$ & $\mathrm{O}$ & $\mathrm{O}$ & $\mathrm{O}$ & O \\
\hline $\begin{array}{l}\text { 28. Wenn ich lerne, versuche ich mein Bestes zu } \\
\text { geben, um mir das Wissen und die Fertigkeiten } \\
\text { anzueignen. }\end{array}$ & $\mathrm{O}$ & $\mathrm{O}$ & $\mathrm{O}$ & $\mathrm{O}$ & $\mathrm{O}$ & $\mathrm{O}$ \\
\hline $\begin{array}{l}\text { 29. Ich bin überzeugt, dass ich die Fertigkeiten, die } \\
\text { gelehrt werden, beherrschen kann. }\end{array}$ & $\mathrm{O}$ & $\mathrm{O}$ & $\mathrm{O}$ & $\mathrm{O}$ & $\mathrm{O}$ & $\mathrm{O}$ \\
\hline $\begin{array}{l}\text { 30. Ich bin mir sicher, dass ich auch noch meine } \\
\text { gewünschten Leistungen erreichen kann, wenn } \\
\text { ich mal eine schlechte Note bekommen habe. }\end{array}$ & $\mathrm{O}$ & O & $\mathrm{O}$ & $\mathrm{O}$ & $\mathrm{O}$ & O \\
\hline $\begin{array}{l}\text { 31. Wenn ich mich hinsetze, um etwas sehr } \\
\text { gründlich zu lernen, dann gelingt es mir auch. }\end{array}$ & $\mathrm{O}$ & $\mathrm{O}$ & $\mathrm{O}$ & $\mathrm{O}$ & $\mathrm{O}$ & O \\
\hline $\begin{array}{l}\text { 32. Ich lerne, damit ich in der Zukunft finanziell } \\
\text { abgesichert sein werde. }\end{array}$ & $\mathrm{O}$ & $\mathrm{O}$ & O & $\mathrm{O}$ & $\mathrm{O}$ & O \\
\hline $\begin{array}{l}\text { 33. Wenn ich lerne, zwinge ich mich zu prüfen, ob } \\
\text { ich das Gelernte auch behalten habe. }\end{array}$ & $\mathrm{O}$ & $\mathrm{O}$ & O & $\mathrm{O}$ & $\mathrm{O}$ & O \\
\hline 34. Mathematik ist mir persönlich wichtig. & $\mathrm{O}$ & $\mathrm{O}$ & O & $\mathrm{O}$ & 0 & 0 \\
\hline $\begin{array}{l}\text { 35. Wenn ich etwas gut lernen will, kann ich das } \\
\text { auch. }\end{array}$ & 0 & 0 & 0 & 0 & 0 & 0 \\
\hline
\end{tabular}




$\begin{array}{ll}\begin{array}{l}\text { trifft } \\ \text { gar nicht zu }\end{array} & \text { trifft } \\ \text { vollständig zu }\end{array}$

36. Weil mir die Beschäftigung mit Mathematik Spaß macht, würde ich das nicht gerne aufgeben.

37. Wenn der Lehrer/die Lehrerin das Tempo noch mehr anzieht, werde ich die geforderten Leistungen kaum noch schaffen können.

38. Ich bin sicher, dass ich auch den schwierigsten Stoff in Unterrichtstexten verstehen kann.

39. Wenn ich lerne, arbeite ich auch dann weiter, wenn der Stoff schwierig ist.

40. Wenn ich mich mit Mathematik beschäftige, vergesse ich manchmal alles um mich herum.

41. Wenn ich eine schwierige Aufgabe an der Tafel lösen soll, glaube ich, dass ich das schaffen werde.

o 0 o 0000

$\begin{array}{llllll}0 & 0 & 0 & 0 & 0 & 0\end{array}$




\title{
Anhang H: Fragebogen 3 - Studie 1
}

\author{
@ \\ GEORG-AUGUST-UNIVERSITÄT \\ GÖTTINGEN
}

\section{Hinweise zur Bearbeitung des Fragebogens}

In diesem Fragebogen geht es um die Dinge, die Ihnen in der Schule wichtig sind. Bitte lesen Sie jede Aussage genau durch und geben Sie an, inwieweit sie auf Sie persönlich zutrifft. Nur Ihre persönliche Meinung zählt - es gibt weder richtige noch falsche Antworten.

Es gibt jeweils fünf Antwortmöglichkeiten, mit deren Hilfe Sie angeben können, wie sehr ein Satz auf Sie zutrifft. Kreuzen Sie bitte das Kästchen an, welches am besten passt!

Bitte nehmen Sie Ihre Markierung stets eindeutig vor. Markierungen zwischen zwei Zahlen können nicht ausgewertet werden. Haben Sie versehentlich eine falsche Markierung vorgenommen, so kreisen Sie diese bitte ein und setzen Sie eine neue Markierung an einer anderen Stelle.

Wichtig ist, dass Sie jede Aussage bearbeiten. Fragebogen mit unbewerteten Aussagen können nicht ausgewertet werden. Wählen Sie im Zweifelsfall diejenige Antwortmöglichkeit, die noch am ehesten auf Sie zutrifft.

Bearbeiten Sie den Fragebogen bitte zügig, aber sorgfältig; halten Sie sich nicht zu lange an einzelnen Aussagen auf.

Tragen Sie bitte hier Ihren persönlichen Code ein.

CODE: 


\begin{tabular}{|c|c|c|c|c|c|}
\hline & $\begin{array}{l}\text { stimmt } \\
\text { gar } \\
\text { nicht }\end{array}$ & $\begin{array}{l}\text { stimmt } \\
\text { eher } \\
\text { nicht }\end{array}$ & $\begin{array}{c}\text { weder/ } \\
\text { noch }\end{array}$ & $\begin{array}{l}\text { stimmt } \\
\text { eher }\end{array}$ & $\begin{array}{l}\text { stimmt } \\
\text { genau }\end{array}$ \\
\hline $\begin{array}{l}\text { 1. In der Schule geht es mir darum, neue Ideen zu } \\
\text { bekommen. }\end{array}$ & 0 & 0 & 0 & O & 0 \\
\hline $\begin{array}{l}\text { 2. Die meisten Dinge, die ich mir vornehme, setze ich } \\
\text { auch um. }\end{array}$ & 0 & 0 & 0 & 0 & 0 \\
\hline $\begin{array}{l}\text { 3. In der Schule geht es mir darum, keine schwierigen } \\
\text { Tests oder Arbeiten zu haben. }\end{array}$ & 0 & 0 & 0 & 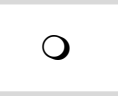 & 0 \\
\hline $\begin{array}{l}\text { 4. Meine Gedanken schweifen oft von der Sache ab, auf } \\
\text { die ich mich eigentlich konzentrieren möchte. }\end{array}$ & 0 & 0 & 0 & 0 & 0 \\
\hline $\begin{array}{l}\text { 5. Ich kann ganz gezielt an heitere Dinge denken, um } \\
\text { lockerer voranzukommen. }\end{array}$ & 0 & 0 & 0 & 0 & 0 \\
\hline $\begin{array}{l}\text { 6. In der Schule geht es mir darum, komplizierte Inhalte } \\
\text { zu verstehen. }\end{array}$ & 0 & 0 & O & 0 & 0 \\
\hline $\begin{array}{l}\text { 7. Nach unangenehmen Erlebnissen kann ich Gedanken } \\
\text { nicht loswerden, die mir die Energie nehmen. }\end{array}$ & 0 & 0 & 0 & 0 & 0 \\
\hline $\begin{array}{l}\text { 8. In der Schule geht es mir darum, zu Hause keine } \\
\text { Arbeiten erledigen zu müssen. }\end{array}$ & 0 & 0 & 0 & 0 & 0 \\
\hline $\begin{array}{l}\text { 9. Wenn etwas getan werden muss, beginne ich damit, } \\
\text { ohne zu zögern. }\end{array}$ & 0 & 0 & 0 & 0 & 0 \\
\hline $\begin{array}{l}\text { 10. Ich kann es schaffen, einer anfangs unangenehmen } \\
\text { Tätigkeit zunehmend angenehme Seiten } \\
\text { abzugewinnen. }\end{array}$ & 0 & 0 & O & 0 & 0 \\
\hline $\begin{array}{l}\text { 11. In der Schule geht es mir darum, so viel wie möglich } \\
\text { zu lernen. }\end{array}$ & $O$ & 0 & 0 & 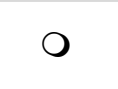 & 0 \\
\hline 12. Ich schiebe viele Dinge vor mir her. & O & O & 0 & O & O \\
\hline 13. Unangenehmes erledige ich oft in letzter Minute. & $\mathrm{O}$ & $\mathrm{O}$ & $\mathrm{O}$ & $\mathrm{O}$ & $\mathrm{O}$ \\
\hline $\begin{array}{l}\text { 14. In der Schule geht es mir darum, keine schwierigen } \\
\text { Fragen oder Aufgaben lösen zu müssen. }\end{array}$ & $\mathrm{O}$ & $\mathrm{O}$ & O & O & $\mathrm{O}$ \\
\hline $\begin{array}{l}\text { 15. Auch wenn Probleme auftauchen, bin ich mir meist } \\
\text { sicher, dass es irgendwie klappt. }\end{array}$ & $\mathrm{O}$ & $\mathrm{O}$ & O & O & $\mathrm{O}$ \\
\hline $\begin{array}{l}\text { 16. Meist ist mir klar, dass das, was ich tue, auch das ist, } \\
\text { was ich selber will. }\end{array}$ & $\mathrm{O}$ & O & O & O & $\mathrm{O}$ \\
\hline $\begin{array}{l}\text { 17. Wenn eine Aufgabe erledigt werden muss, packe ich } \\
\text { sie am liebsten sofort an. }\end{array}$ & $\mathrm{O}$ & $\mathrm{O}$ & O & O & $\mathrm{O}$ \\
\hline $\begin{array}{l}\text { 18. In der Schule geht es mir darum, etwas Interessantes } \\
\text { zu lernen. }\end{array}$ & $\mathrm{O}$ & $\mathrm{O}$ & O & $\mathrm{O}$ & O \\
\hline 19. Ich habe mich selbst ganz gut im Griff. & $\mathrm{O}$ & $\mathrm{O}$ & $\mathrm{O}$ & $\mathrm{O}$ & $\mathrm{O}$ \\
\hline $\begin{array}{l}\text { 20. Dinge, die ich noch nicht erledigt habe, mache ich } \\
\text { mir immer wieder bewusst. }\end{array}$ & $\mathrm{O}$ & $\mathrm{O}$ & $\mathrm{O}$ & O & $\mathrm{O}$ \\
\hline $\begin{array}{l}\text { 21. In der Schule geht es mir darum, nicht so schwer zu } \\
\text { arbeiten. }\end{array}$ & $\mathrm{O}$ & $\mathrm{O}$ & O & O & $\mathrm{O}$ \\
\hline $\begin{array}{l}\text { 22. Ich kann meine Stimmung so verändern, dass mir } \\
\text { dann alles leichter von der Hand geht. }\end{array}$ & $\mathrm{O}$ & $\mathrm{O}$ & O & O & O \\
\hline $\begin{array}{l}\text { 23. Ich erreiche meine beste Form erst dann, wenn } \\
\text { Schwierigkeiten auftauchen. }\end{array}$ & $\mathrm{O}$ & $\mathrm{O}$ & O & O & $\mathrm{O}$ \\
\hline 24. In der Schule geht es mir darum, dass die Arbeit leicht ist. & $\mathrm{O}$ & $\mathrm{O}$ & O & $\mathrm{O}$ & $\mathrm{O}$ \\
\hline 25. Ich fühle mich oft ziemlich lustlos. & $\mathrm{O}$ & $\mathrm{O}$ & $\mathrm{O}$ & $\mathrm{O}$ & $\mathrm{O}$ \\
\hline $\begin{array}{l}\text { 26. Auch in schwierigen Situationen vertraue ich darauf, } \\
\text { dass ich die Probleme irgendwie bewältigen werde. }\end{array}$ & 0 & 0 & 0 & 0 & 0 \\
\hline $\begin{array}{l}\text { 27. In der Schule geht es mir darum, aufwendige } \\
\text { Aufgaben nicht selber erledigen zu müssen. }\end{array}$ & $\mathrm{O}$ & $\mathrm{O}$ & $\mathrm{O}$ & O & $\mathrm{O}$ \\
\hline
\end{tabular}




\begin{tabular}{|c|c|c|c|c|c|c|}
\hline & & $\begin{array}{l}\text { stimmt } \\
\text { gar } \\
\text { nicht }\end{array}$ & $\begin{array}{l}\text { stimmt } \\
\text { eher } \\
\text { nicht }\end{array}$ & $\begin{array}{c}\text { weder/ } \\
\text { noch }\end{array}$ & $\begin{array}{c}\text { stimmt } \\
\text { eher }\end{array}$ & $\begin{array}{r}\text { stimmt } \\
\text { genau }\end{array}$ \\
\hline & $\begin{array}{l}\text { Wenn eine Sache langweilig wird, weiß ich meist, } \\
\text { wie ich wieder Spaß daran finden kann. }\end{array}$ & 0 & 0 & O & 0 & 0 \\
\hline & $\begin{array}{l}\text { In der Schule geht es mir darum, zum Nachdenken } \\
\text { angeregt zu werden. }\end{array}$ & 0 & 0 & O & 0 & 0 \\
\hline 30. & Ich habe oft zu wenig Energie. & 0 & 0 & 0 & 0 & 0 \\
\hline & $\begin{array}{l}\text { Sobald Hindernisse auftauchen, spüre ich, wie ich } \\
\text { aktiver werde. }\end{array}$ & 0 & 0 & 0 & 0 & 0 \\
\hline & $\begin{array}{l}\text { Wenn ich ein Ziel nicht erreiche, verliere ich oft } \\
\text { jeden Schwung. }\end{array}$ & 0 & 0 & 0 & 0 & 0 \\
\hline 33. & $\begin{array}{l}\text { In der Schule geht es mir darum, mit wenig Arbeit } \\
\text { durch die Schule zu kommen. }\end{array}$ & 0 & 0 & 0 & 0 & 0 \\
\hline 34. & $\begin{array}{l}\text { Oft muss ich an Dinge denken, die mit dem, was ich } \\
\text { gerade tue, gar nichts zu tun haben. }\end{array}$ & 0 & 0 & 0 & 0 & 0 \\
\hline 35. & $\begin{array}{l}\text { Bei meinen Handlungen spüre ich meist, dass ich es } \\
\text { bin, der so handeln will. }\end{array}$ & 0 & 0 & $\mathrm{O}$ & $\mathrm{O}$ & 0 \\
\hline 36. & $\begin{array}{l}\text { Wenn eine Versuchung auftaucht, kann ich schlecht } \\
\text { nein sagen. }\end{array}$ & 0 & 0 & 0 & 0 & 0 \\
\hline 37. & Ich schiebe unangenehme Dinge oft auf. & $\mathrm{O}$ & $\mathrm{O}$ & $\mathrm{O}$ & 0 & 0 \\
\hline & $\begin{array}{l}\text { In der Schule geht es mir darum, später knifflige } \\
\text { Probleme lösen zu können. }\end{array}$ & 0 & 0 & 0 & 0 & 0 \\
\hline 39. & $\begin{array}{l}\text { Wenn ich etwas erreichen will, setze ich mir ein ganz } \\
\text { genaues Ziel. }\end{array}$ & 0 & 0 & O & 0 & 0 \\
\hline 40. & $\begin{array}{l}\text { Ich habe oft ein Ziel, ohne zu wissen, wie ich es } \\
\text { erreichen kann. }\end{array}$ & 0 & 0 & 0 & 0 & O \\
\hline 41. & Ich verfolge meist sehr genaue Ziele. & $\mathrm{O}$ & $\mathrm{O}$ & $\mathrm{O}$ & $\mathrm{O}$ & 0 \\
\hline & $\begin{array}{l}\text { In der Schule geht es mir darum, dass das Gelernte } \\
\text { für mich einen Sinn ergibt. }\end{array}$ & 0 & 0 & 0 & 0 & 0 \\
\hline & $\begin{array}{l}\text { Bei schwierigen Aufgaben kann ich eine enorme } \\
\text { Ausdauer entwickeln. }\end{array}$ & 0 & 0 & O & 0 & 0 \\
\hline & $\begin{array}{l}\text { In der Schule geht es mir darum, den Arbeitsaufwand } \\
\text { stets gering zu halten. }\end{array}$ & 0 & 0 & 0 & $\mathrm{O}$ & O \\
\hline & $\begin{array}{l}\text { Ich lasse die Dinge lieber auf mich zukommen als } \\
\text { genau zu planen. }\end{array}$ & 0 & 0 & 0 & 0 & 0 \\
\hline & $\begin{array}{l}\text { Wenn ich mir einmal ein Ziel gesetzt habe, lasse ich } \\
\text { mich nicht leicht davon abbringen. }\end{array}$ & 0 & 0 & 0 & 0 & O \\
\hline & $\begin{array}{l}\text { Wenn Schwierigkeiten auftauchen, kann ich eine } \\
\text { enorme Hartnäckigkeit entwickeln. }\end{array}$ & 0 & $\mathrm{O}$ & 0 & 0 & 0 \\
\hline & $\begin{array}{l}\text { In der Schule geht es mir darum, ein tiefes } \\
\text { Verständnis für die Inhalte zu erwerben. }\end{array}$ & 0 & 0 & 0 & 0 & 0 \\
\hline
\end{tabular}




\section{Anhang I: Fragebogen - Studie 2}

\section{Hinweise zur Bearbeitung des Fragebogens}

Bitte lesen Sie jede Aussage genau durch und geben Sie an, inwieweit diese auf Sie persönlich zutrifft. Nur Ihre persönliche Meinung zählt - es gibt weder richtige noch falsche Antworten!

Es gibt jeweils mehrere Antwortmöglichkeiten, mit deren Hilfe Sie angeben können, wie sehr ein Satz auf Sie zutrifft. Trifft die Aussage für Sie gar nicht zu, dann setzen Sie ein Kreuz im ersten Kästchen links. Trifft die Aussage für Sie vollständig zu, setzen Sie Ihr Kreuz im Kästchen ganz rechts. Für verschiedene Abstufungen kreuzen sie bitte die Kästchen dazwischen an. Bitte beachten Sie, dass die Anzahl der Antwortmöglichkeiten in diesem Fragebogen variiert.

Nehmen Sie Ihre Markierung bitte stets eindeutig vor. Markierungen zwischen zwei Zahlen können nicht ausgewertet werden. Haben Sie versehentlich eine falsche Markierung vorgenommen, so streichen Sie diese bitte deutlich durch und setzen Ihre neue Markierung an einer anderen Stelle.

Wichtig ist, dass Sie jede Aussage bearbeiten. Fragebogen mit unbewerteten Aussagen können nicht ausgewertet werden. Wählen Sie im Zweifelsfall diejenige Antwortmöglichkeit, die noch am ehesten auf Sie zutrifft.

Bearbeiten Sie den Fragebogen bitte zügig, aber sorgfältig. Halten Sie sich nicht zu lange an einzelnen Aussagen auf. 


\begin{tabular}{|c|c|c|c|}
\hline & & $\begin{array}{l}\text { Trifft } \\
\text { gar nicht } \\
\text { zu }\end{array}$ & $\begin{array}{r}\text { Trifft } \\
\text { vollständig } \\
\text { zu }\end{array}$ \\
\hline 1. & $\begin{array}{l}\text { Ich bin überzeugt davon, dass ich es beruflich zu etwas } \\
\text { bringen werde. }\end{array}$ & (1) (2) (3) (4) (5) & (6) (7) \\
\hline 2. & $\begin{array}{l}\text { Wenn ich eine Prüfung ablege, bin ich auch davon } \\
\text { überzeugt, dass ich sie bestehe. }\end{array}$ & (1) (2) (3) (4) (5) & (6) (7) \\
\hline 3. & $\begin{array}{l}\text { Ich bin zuversichtlich, dass meine Leistung die Aner- } \\
\text { kennung anderer finden wird. }\end{array}$ & (1) (2) (3) (4) (5) & (6) (7) \\
\hline 4. & $\begin{array}{l}\text { Auch wenn ich vor schwierigen Aufgaben stehe, bin ich } \\
\text { immer guten Mutes. }\end{array}$ & (1) (2) (3) (4) (5) & (6) 7 \\
\hline 5. & $\begin{array}{l}\text { Ich brauche mich vor keiner Situation zu fürchten, weil } \\
\text { ich mit meinen Fähigkeiten noch überall durch- } \\
\text { gekommen bin. }\end{array}$ & (1) (2) (3) (4) (5) & (6) (7) \\
\hline 6. & $\begin{array}{l}\text { Auch vor einer schwierigen Aufgabe rechne ich immer } \\
\text { damit, mein Ziel zu erreichen. }\end{array}$ & (1) (2) (3) (4) (5) & (6) (7) \\
\hline 7. & $\begin{array}{l}\text { Vor neuen Aufgaben war ich immer zuversichtlich, sie } \\
\text { zu schaffen. }\end{array}$ & (1) (2) (3) (4) (5) & (6) 7 \\
\hline & $\begin{array}{l}\text { Wenn ich mir etwas vornehme, dann gelingt es mir } \\
\text { meist besser als anderen Leuten. }\end{array}$ & (1) (2) (3) (4) & \\
\hline
\end{tabular}

Bitte beachten Sie, dass im Folgenden nur noch zwischen 6 Abstufungen unterschieden wird!

\begin{tabular}{|c|c|c|c|c|c|c|}
\hline & \multicolumn{2}{|c|}{$\begin{array}{l}\text { Trifft } \\
\text { gar nicht } \\
\text { zu }\end{array}$} & & \multicolumn{3}{|c|}{$\begin{array}{r}\text { Trifft } \\
\text { vollständig } \\
\text { zu }\end{array}$} \\
\hline $\begin{array}{l}\text { 9. Wenn ich lerne, versuche ich alles auswendig zu lernen, } \\
\text { was drankommen könnte. }\end{array}$ & 0 & 0 & 0 & 0 & 0 & 0 \\
\hline $\begin{array}{l}\text { 10. Weil mir die Beschäftigung mit Mathematik Spaß macht, } \\
\text { würde ich das nicht gerne aufgeben. }\end{array}$ & 0 & 0 & 0 & 0 & 0 & 0 \\
\hline $\begin{array}{l}\text { 11. Wenn ich lerne, überlege ich, inwiefern die Information } \\
\text { im wirklichen Leben nützlich sein könnte. }\end{array}$ & 0 & 0 & 0 & 0 & 0 & 0 \\
\hline $\begin{array}{l}\text { 12. Wenn ich lerne, versuche ich, neuen Stoff mit Dingen zu } \\
\text { verbinden, die ich in anderen Fächern gelernt habe. }\end{array}$ & 0 & 0 & 0 & 0 & 0 & 0 \\
\hline $\begin{array}{l}\text { 13. Wenn ich lerne und etwas nicht verstehe, suche ich nach } \\
\text { zusätzlicher Information, um das Problem zu klären. }\end{array}$ & 0 & $\mathrm{O}$ & 0 & 0 & 0 & 0 \\
\hline $\begin{array}{l}\text { 14. Wenn ich mich mit Mathematik beschäftige, vergesse ich } \\
\text { manchmal alles um mich herum. }\end{array}$ & 0 & 0 & 0 & 0 & 0 & 0 \\
\hline $\begin{array}{l}\text { 15. Wenn ich lerne, versuche ich den Stoff besser zu } \\
\text { verstehen, indem ich Verbindungen zu Dingen herstelle, } \\
\text { die ich schon kenne. }\end{array}$ & 0 & 0 & 0 & 0 & 0 & 0 \\
\hline 16. Wenn ich lerne, lerne ich so viel wie möglich auswendig. & O & O & 0 & 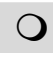 & O & O \\
\hline $\begin{array}{l}\text { 17. Wenn ich lerne, überlege ich, wie der Stoff mit dem } \\
\text { zusammenhängt, was ich schon gelernt habe. }\end{array}$ & O & $\mathrm{O}$ & 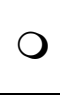 & כ & 0 & D \\
\hline
\end{tabular}




\section{Trifft}

Zu

$\mathbf{z u}$

18. Wenn ich lerne, versuche ich beim Lesen herauszufinden, was ich noch nicht richtig verstanden habe.

19. Wenn ich mir vornehme, keine schlechten Noten zu bekommen, dann gelingt es mir.

20. Wenn ich lerne, überlege ich mir zuerst, was genau ich lernen muss.

21. Wenn ich lerne, präge ich mir alles Neue so ein, dass ich es aufsagen kann.

22. Wenn ich mich hinsetze, um etwas sehr gründlich zu lernen, dann gelingt es mir auch.

23. Wenn ich lerne, zwinge ich mich zu prüfen, ob ich das Gelernte auch behalten habe.

24. Mathematik ist mir persönlich wichtig.

25. Wenn ich etwas gut lernen will, kann ich das auch.

26. Wenn ich lerne, passe ich genau auf, dass ich das Wichtigste behalte.

27. Wenn ich mir vornehme, bei keiner Aufgabe einen Fehler zu machen, gelingt es mir.

28. Wenn ich lerne, übe ich, indem ich den Stoff immer wieder aufsage.

$\begin{array}{llllll}0 & 0 & 0 & 0 & 0 & 0 \\ 0 & 0 & 0 & 0 & 0 & 0 \\ 0 & 0 & 0 & 0 & 0 & 0 \\ 0 & 0 & 0 & 0 & 0 & 0 \\ 0 & 0 & 0 & 0 & 0 & 0 \\ 0 & 0 & 0 & 0 & 0 & 0\end{array}$

Im Folgenden geht es speziell um das Lernen in den Fächern Mathematik, Deutsch und Englisch. Bitte lesen Sie sich jede Aussage sorgfältig durch. Schätzen Sie dann ein, wie gut jede Aussage auf Sie zutrifft, und zwar getrennt für das jeweilige Fach. In jeder Aussage ist „in ... “ enthalten. Sie sollen also dort in Gedanken das vorgegebene Fach (Mathematik oder Deutsch oder Englisch) beim Lesen einsetzen. Die Antwortmöglichkeiten reichen von „1= trifft gar nicht zu“ bis „6= trifft vollständig zu“. Kreuzen Sie bitte die entsprechende Antwort an.

Bitte beachten Sie, dass im Folgenden nur noch zwischen 5 Abstufungen unterschieden wird!

\begin{tabular}{|c|c|c|c|}
\hline & Mathematik & Deutsch & Englisch \\
\hline & 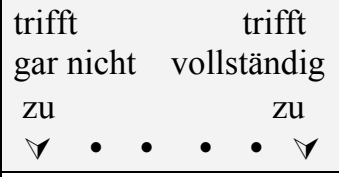 & 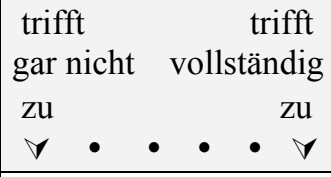 & 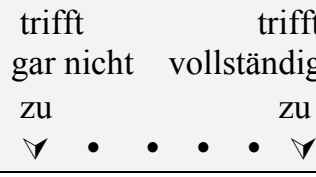 \\
\hline $\begin{array}{l}\text { Ich weiß in ... die Antwort auf eine } \\
\text { Frage schneller als die Anderen. }\end{array}$ & (1) (2) (3) (4) (5) (6) & (1)-(2)-(3)-(4)-(5)-(6) & (1)-(2)-(3)-(4)-(5)-(6) \\
\hline $\begin{array}{l}\text { Manchmal fühle ich mich in ... anderen } \\
\text { überlegen und glaube, dass sie noch } \\
\text { manches von mir lernen können. }\end{array}$ & (1)-(2)-(3)-(4)-(5)-(6) & (1)-(2)-(3)-(4)-(5)-(6) & (1)-(2)-(3)-(4)-(5)-(6) \\
\hline $\begin{array}{l}\text { Ich kann in ... Sachen selbst } \\
\text { rauskriegen. }\end{array}$ & (1)-(2)-(3)-(4)-(5)-(6) & (1)-(2)-(3)-(4)-(5)-(6) & (1)-(2)-(3)-(4)-(5)-(6) \\
\hline $\begin{array}{l}\text { Ich bin in ... zufrieden mit me } \\
\text { Fähigkeit, vor der Klasse zu s }\end{array}$ & (1)-(2)-(3)-(4)-(5)-(6) & (1)-(2)-(3)-(4)-(5)-(6) & (1)-(2)-(3)-(4)-(5)-(6) \\
\hline $\begin{array}{l}\text { Es fällt mir in ... leicht, Probleme zu } \\
\text { lösen. }\end{array}$ & (1)-(2)-(3)-(4)-(5)-(6) & (1)-(2)-(3)-(4)-(5)-(6) & (1)-(2)-(3)-(4)-(5)-(6) \\
\hline In ... fallen mir gute Noten zu. & (1)-(2)-(3)-(4)-(5)-(6) & (1)-(2)-(3)-(4)-(5)-(6) & (1)-(2)-(3)-(4)-(5)-(6) \\
\hline Ich gehöre in ... zu den Guten. & (1)-(2)-(3)-(4)-(5)-(6) & (1)-(2)-(3)-(4)-(5)-(6) & (1)-(2)-(3)-(4)-(5)-(6) \\
\hline $\begin{array}{l}\text { Ich habe ein gutes Gefühl, was } m \\
\text { Arbeit in ... angeht. }\end{array}$ & (1)-(2)-(3)-(4)-(5)-(6) & (1)-(2)-(3)-(4)-(5)-(6) & (1)-(2)-(3)-(4)-(5)-(6) \\
\hline
\end{tabular}




\begin{tabular}{|c|c|c|c|c|c|}
\hline & $\begin{array}{l}\text { stimmt } \\
\text { gar } \\
\text { nicht }\end{array}$ & $\begin{array}{l}\text { stimmt } \\
\text { eher } \\
\text { nicht }\end{array}$ & $\begin{array}{l}\text { weder/ } \\
\text { noch }\end{array}$ & $\begin{array}{l}\text { stimmt } \\
\text { eher }\end{array}$ & $\begin{array}{l}\text { stimmt } \\
\text { genau }\end{array}$ \\
\hline $\begin{array}{l}\text { 29. In der Schule geht es mir darum, neue Ideen zu } \\
\text { bekommen. }\end{array}$ & $\mathrm{O}$ & $\mathrm{O}$ & $\mathrm{O}$ & $\mathrm{O}$ & O \\
\hline $\begin{array}{l}\text { 30. In der Schule geht es mir darum, keine } \\
\text { schwierigen Tests oder Arbeiten zu haben. }\end{array}$ & $\mathrm{O}$ & 0 & O & O & O \\
\hline $\begin{array}{l}\text { 31. In der Schule geht es mir darum, komplizierte } \\
\text { Inhalte zu verstehen. }\end{array}$ & $\mathrm{O}$ & O & $\mathrm{O}$ & $\mathrm{O}$ & O \\
\hline $\begin{array}{l}\text { 32. In der Schule geht es mir darum, zu Hause keine } \\
\text { Arbeiten erledigen zu müssen. }\end{array}$ & $\mathrm{O}$ & O & O & O & $\mathrm{O}$ \\
\hline $\begin{array}{l}\text { 33. Wenn etwas getan werden muss, beginne ich } \\
\text { damit, ohne zu zögern. }\end{array}$ & $\mathrm{O}$ & $\mathrm{O}$ & $\mathrm{O}$ & $\mathrm{O}$ & $\mathrm{O}$ \\
\hline $\begin{array}{l}\text { 34. In der Schule geht es mir darum, so viel wie } \\
\text { möglich zu lernen. }\end{array}$ & $\mathrm{O}$ & O & O & O & O \\
\hline 35. Ich schiebe viele Dinge vor mir her. & O & 0 & O & O & O \\
\hline $\begin{array}{l}\text { 36. In der Schule geht es mir darum, keine } \\
\text { schwierigen Fragen oder Aufgaben lösen zu } \\
\text { müssen. }\end{array}$ & O & $\mathrm{O}$ & O & O & $\mathrm{O}$ \\
\hline $\begin{array}{l}\text { 37. Wenn eine Aufgabe erledigt werden muss, packe } \\
\text { ich sie am liebsten sofort an. }\end{array}$ & $\mathrm{O}$ & $\mathrm{O}$ & $\mathrm{O}$ & $\mathrm{O}$ & $\mathrm{O}$ \\
\hline $\begin{array}{l}\text { 38. In der Schule geht es mir darum, etwas } \\
\text { Interessantes zu lernen. }\end{array}$ & O & O & O & O & O \\
\hline $\begin{array}{l}\text { 39. In der Schule geht es mir darum, nicht so schwer } \\
\text { zu arbeiten. }\end{array}$ & $\mathrm{O}$ & O & $\mathrm{O}$ & $\mathrm{O}$ & O \\
\hline $\begin{array}{l}\text { 40. In der Schule geht es mir darum, dass die Arbeit } \\
\text { leicht ist. }\end{array}$ & O & O & O & O & O \\
\hline $\begin{array}{l}\text { 41. In der Schule geht es mir darum, zum } \\
\text { Nachdenken angeregt zu werden. }\end{array}$ & $\mathrm{O}$ & O & $\mathrm{O}$ & O & O \\
\hline $\begin{array}{l}\text { 42. In der Schule geht es mir darum, mit wenig } \\
\text { Arbeit durch die Schule zu kommen. }\end{array}$ & $\mathrm{O}$ & O & O & O & O \\
\hline $\begin{array}{l}\text { 43. Wenn eine Versuchung auftaucht, kann ich } \\
\text { schlecht nein sagen. }\end{array}$ & $\mathrm{O}$ & O & $\mathrm{O}$ & O & O \\
\hline $\begin{array}{l}\text { 44. In der Schule geht es mir darum, aufwendige } \\
\text { Aufgaben nicht selber erledigen zu müssen. }\end{array}$ & O & $\bigcirc$ & O & O & $\mathrm{O}$ \\
\hline 45. Ich schiebe unangenehme Dinge oft auf. & $\mathrm{O}$ & O & O & O & O \\
\hline $\begin{array}{l}\text { 46. In der Schule geht es mir darum, später knifflige } \\
\text { Probleme lösen zu können. }\end{array}$ & $\mathrm{O}$ & O & $\mathrm{O}$ & $\mathrm{O}$ & O \\
\hline $\begin{array}{l}\text { 47. In der Schule geht es mir darum, dass das } \\
\text { Gelernte für mich einen Sinn ergibt. }\end{array}$ & $\mathrm{O}$ & O & $\mathrm{O}$ & $\mathrm{O}$ & O \\
\hline $\begin{array}{l}\text { 48. In der Schule geht es mir darum, den } \\
\text { Arbeitsaufwand stets gering zu halten. }\end{array}$ & $\mathrm{O}$ & O & $\mathrm{O}$ & $\mathrm{O}$ & $\mathrm{O}$ \\
\hline $\begin{array}{l}\text { 49. In der Schule geht es mir darum, ein tiefes } \\
\text { Verständnis für die Inhalte zu erwerben. }\end{array}$ & 0 & 0 & $\mathrm{O}$ & 0 & 0 \\
\hline $\begin{array}{l}\text { 50. Unangenehmes erledige ich oft erst in letzter } \\
\text { Minute. }\end{array}$ & 0 & 0 & 0 & 0 & 0 \\
\hline
\end{tabular}




\section{Anhang J: Skalen aus dem Pisa-Fragebogen zum Selbst- regulierten Lernen, Baumert et al. (2000)}

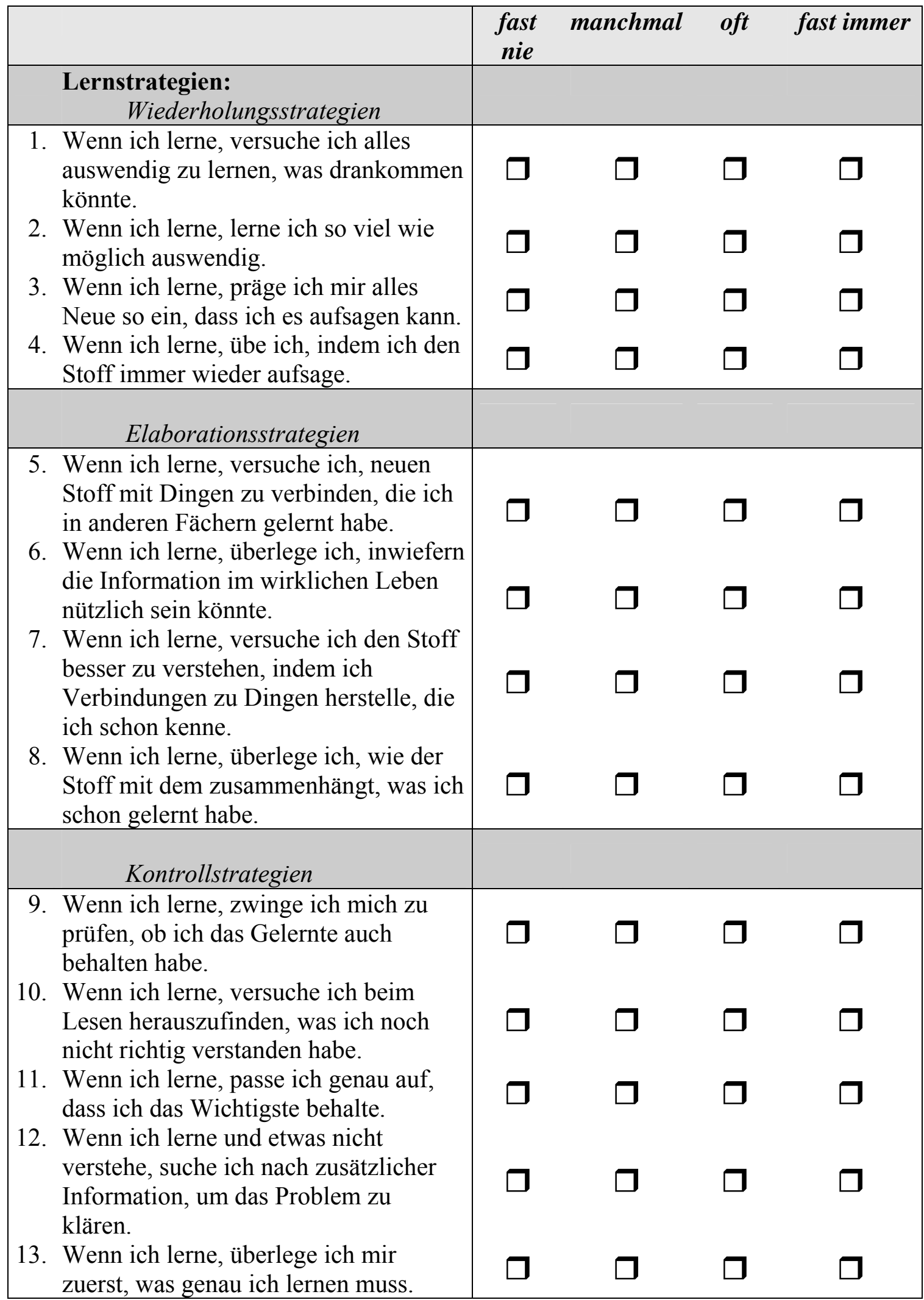




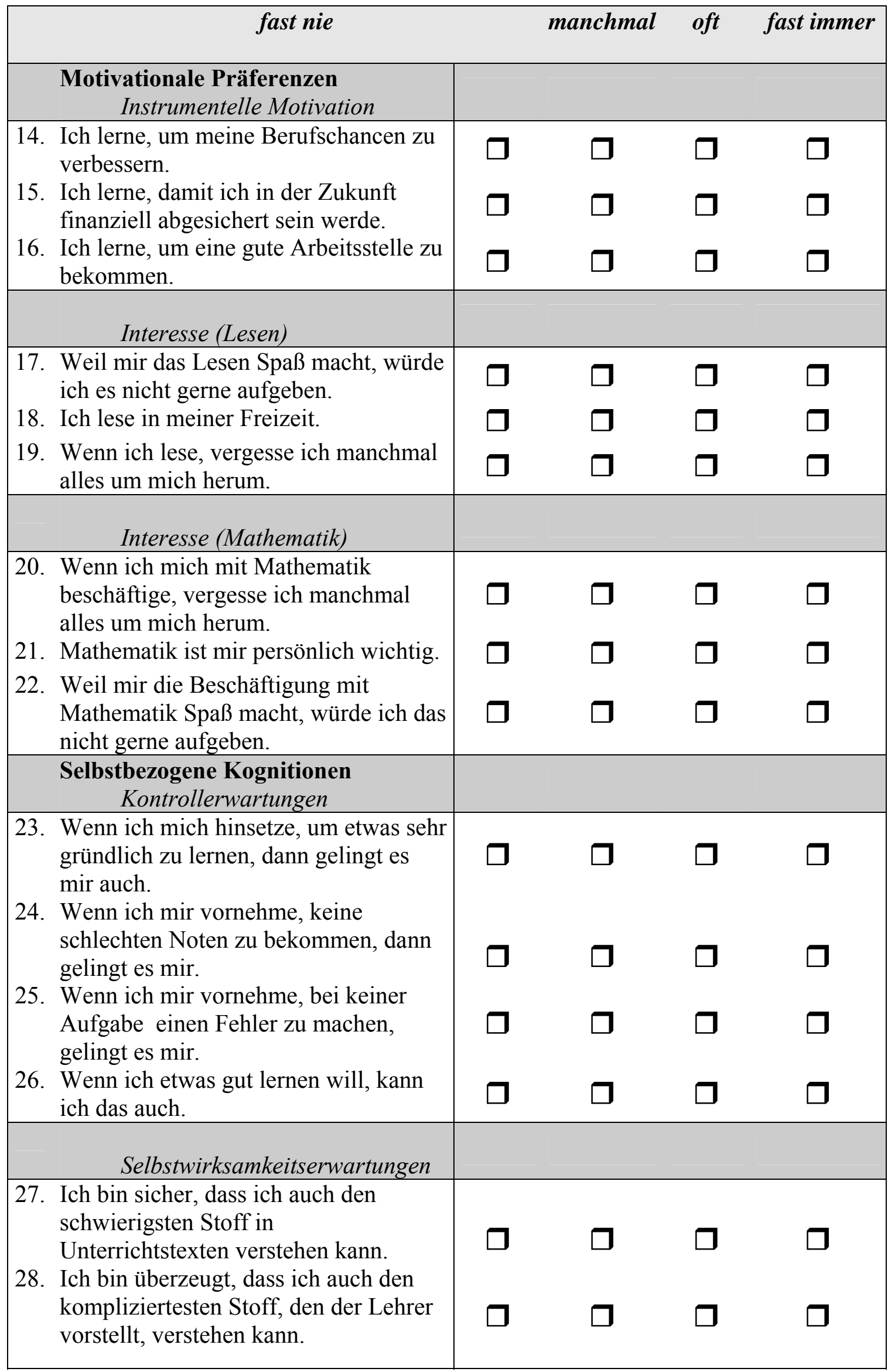




\begin{tabular}{|c|c|c|c|c|}
\hline & $\begin{array}{c}\text { fast } \\
\text { nie }\end{array}$ & manchmal & oft & fast immer \\
\hline $\begin{array}{l}\text { 29. Ich bin überzeugt, dass ich in } \\
\text { Hausaufgaben und Klassenarbeiten } \\
\text { gute Leistungen erzielen kann. } \\
\text { 30. Ich bin überzeugt, dass ich die } \\
\text { Fertigkeiten, die gelehrt werden, } \\
\text { beherrschen kann. }\end{array}$ & $\square$ & $\square$ & $\square$ & $\square$ \\
\hline $\begin{array}{l}\text { Handlungskontrolle } \\
\text { Anstrengung und Ausdauer }\end{array}$ & & & & \\
\hline $\begin{array}{l}\text { 31. Wenn ich lerne, arbeite ich so fleißig } \\
\text { wie möglich. }\end{array}$ & $\square$ & $\square$ & 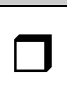 & $\square$ \\
\hline $\begin{array}{l}\text { 32. Wenn ich lerne, arbeite ich auch dann } \\
\text { weiter, wenn der Stoff schwierig ist. } \\
\text { 33. Wenn ich lerne, versuche ich mein }\end{array}$ & $\square$ & $\square$ & $\mathbf{J}$ & 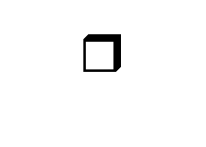 \\
\hline $\begin{array}{l}\text { Bestes zu geben, um mir das Wissen } \\
\text { und die Fertigkeiten anzueignen. } \\
\text { 34. Wenn ich lerne, gebe ich mein Bestes. }\end{array}$ & $\square$ & $\square$ & $\square$ & $\square$ \\
\hline
\end{tabular}




\section{Anhang K: Skala WIRSCHUL zur schulischen Selbstwirk- samkeitserwartung, Jerusalem \& Satow (1999)}

\begin{tabular}{|c|c|c|c|c|}
\hline & $\begin{array}{l}\text { Trifft } \\
\text { nicht zu }\end{array}$ & $\begin{array}{l}\text { Trifft } \\
\text { kaum zu }\end{array}$ & $\begin{array}{l}\text { Trifft } \\
\text { eher zu }\end{array}$ & $\begin{array}{l}\text { Trifft } \\
\text { genau zu }\end{array}$ \\
\hline $\begin{array}{l}\text { 1. Ich kann auch die schwierigen Aufgaben } \\
\text { im Unterricht lösen, wenn ich mich } \\
\text { anstrenge. }\end{array}$ & $\square$ & $\square$ & $\square$ & $\square$ \\
\hline $\begin{array}{l}\text { 2. Es fällt mir leicht, neuen Unterrichtsstoff } \\
\text { zu verstehen. }\end{array}$ & $\square$ & $\square$ & $\square$ & $\square$ \\
\hline $\begin{array}{l}\text { 3. Wenn ich eine schwierige Aufgabe an der } \\
\text { Tafel lösen soll, glaube ich, dass ich das } \\
\text { schaffen werde. }\end{array}$ & $\square$ & $\square$ & $\square$ & $\square$ \\
\hline $\begin{array}{l}\text { 4. Selbst wenn ich mal längere Zeit krank } \\
\text { sein sollte, kann ich immer noch gute } \\
\text { Leistungen erzielen. }\end{array}$ & $\square$ & $\square$ & $\square$ & $\square$ \\
\hline $\begin{array}{l}\text { 5. Wenn der Lehrer/die Lehrerin das Tempo } \\
\text { noch mehr anzieht, werde ich die } \\
\text { geforderten Leistungen kaum noch } \\
\text { schaffen können. }\end{array}$ & $\square$ & $\square$ & $\square$ & $\square$ \\
\hline $\begin{array}{l}\text { 6. Auch wenn der Lehrer/die Lehrerin an } \\
\text { meinen Fähigkeiten zweifelt, bin ich mir } \\
\text { sicher, dass ich gute Leistungen erzielen } \\
\text { kann. }\end{array}$ & $\square$ & $\square$ & $\square$ & $\square$ \\
\hline $\begin{array}{l}\text { 7. Ich bin mir sicher, dass ich auch noch } \\
\text { meine gewünschten Leistungen erreichen } \\
\text { kann, wenn ich mal eine schlechte Note } \\
\text { bekommen habe. }\end{array}$ & $\square$ & $\square$ & $\square$ & $\square$ \\
\hline
\end{tabular}




\section{Anhang L: DISK-Gitter, Rost \& Sparfeldt (2002)}

\begin{tabular}{|c|c|c|c|}
\hline & Mathematik & Deutsch & Englisch \\
\hline & 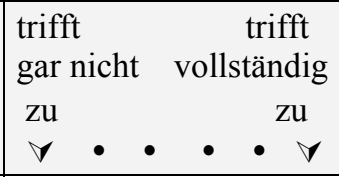 & 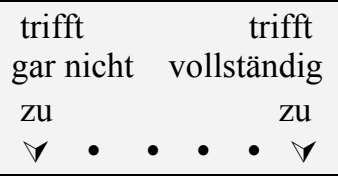 & 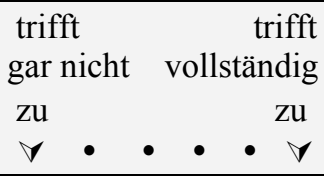 \\
\hline $\begin{array}{l}\text { Ich weiß in ... die Antwort auf eine } \\
\text { Frage schneller als die Anderen. }\end{array}$ & (1)-(2)-(3)-(4)-(5)-(6) & (1)-(2)-(3)-(4)-(5)-(6) & (1)-(2)-(3)-(4)-(5)-(6) \\
\hline $\begin{array}{l}\text { Manchmal fühle ich mich in ... anderen } \\
\text { überlegen und glaube, dass sie noch } \\
\text { manches von mir lernen können. }\end{array}$ & (1)-(2)-(3)-(4)-(5)-(6) & (1)-(2)-(3)-(4)-(5)-(6) & (1)-(2)-(3)-(4)-(5)-(6) \\
\hline $\begin{array}{l}\text { Ich kann in ... Sachen selbst } \\
\text { rauskriegen. }\end{array}$ & (1)-(2)-(3)-(4)-(5)-(6) & (1)-(2)-(3)-(4)-(5)-(6) & (1)-(2)-(3)-(4)-(5)-(6) \\
\hline $\begin{array}{l}\text { zufrieden mit m } \\
\text { or der Klasse zu s }\end{array}$ & (1)-(2)-(3)-(4)-(5)-(6) & (1)-(2)-(3)-(4)-(5)-(6) & (1)-(2)-(3)-(4)-(5)-(6) \\
\hline $\begin{array}{l}\text { Es fällt mir in ... leicht, Probleme zu } \\
\text { lösen. }\end{array}$ & (1)-(2)-(3)-(4)-(5)-(6) & (1)-(2)-(3)-(4)-(5)-(6) & (1)-(2)-(3)-(4)-(5)-(6) \\
\hline In ... fallen mir gute Noten zu. & (1)-(2)-(3)-(4)-(5)-(6) & (1)-(2)-(3)-(4)-(5)-(6) & (1)-(2)-(3)-(4)-(5)-(6) \\
\hline Ich gehöre in ... zu den Guten. & (1)-(2)-(3)-(4)-(5)-(6) & (1)-(2)-(3)-(4)-(5)-(6) & (1)-(2)-(3)-(4)-(5)-(6) \\
\hline $\begin{array}{l}\text { Ich habe ein gutes Gefühl, was meine } \\
\text { Arbeit in ... angeht. }\end{array}$ & -(5)-(6) & -6) & (1)- \\
\hline
\end{tabular}




\section{Anhang M: Leistungsmotivationsinventar (Kurzform), Schuler \& Prochaska (2001)}

Name:

Schule (Schultyp):
Alter:

Klasse:
Geschlecht: $\quad \mathrm{m} \square \mathrm{w}$ Datum:

1. Ich habe mir vorgenommen, es beruflich weit zu bringen.

2. Ich bin überzeugt davon, dass ich es beruflich zu etwas bringen werde.

3. Mir sind Aufgaben lieber, die mir leicht von der Hand gehen, als solche, bei denen ich mich sehr einsetzen muss.

4. Ich bin überzeugt, mich bisher in Ausbildung und Beruf mehr engagiert zu haben als meine Kollegen.

5. Ich empfinde Befriedigung darüber, meine eigene Leistung zu steigern.

6. Aufgaben, bei denen ich nicht ganz sicher bin, ob ich sie lösen kann, reizen mich ganz besonders.

7. Es ist mir sehr wichtig, eine verantwortungsvolle Position zu erreichen.

8. Wenn ich eine Prüfung ablege, bin ich auch davon überzeugt, dass ich sie bestehe.

9. Mein Ehrgeiz ist leicht herauszufordern.

10. Ich beschäftige mich besonders gern mit Problemen, bei denen es eine harte Nuss zu knacken gibt.

11. Ich bin zuversichtlich, dass meine Leistung die Anerkennung anderer finden wird.

12. Ich suche mir gern Aufgaben, an denen ich meine Fähigkeiten prüfen kann.

13. Am glücklichsten bin ich mit einer Aufgabe, bei der ich alle meine Kräfte einsetzen kann.

14. Wenn mir etwas nicht so gut gelungen ist, wie ich es mir vorgenommen hatte, strenge ich mich anschließend noch mehr an.

15. Der Wunsch, besser zu sein als andere, ist ein großer Ansporn für mich.

16. Schwierige Probleme reizen mich mehr als einfache.

17. Auch wenn ich vor schwierigen Aufgaben stehe, bin ich immer guten Mutes.

$$
\begin{aligned}
& \text { trifft trifft } \\
& \text { gar nicht zu vollständig zu } \\
& \text { (1)-(2)-(3)-(4)-(5)-(6)-(7) } \\
& \text { (1)-(2)-(3)-(4)-(5)-(6)-(7) } \\
& \text { (1)-(2)-(3)-(4)-(5)-(6)-(7) } \\
& \text { (1)-(2)-(3)-(4)-(5)-(6)-(7) } \\
& \text { (1)-(2)-(3)-(4)-(5)-(6)-(7) } \\
& \text { (1)-(2)-(3)-(4)-(5)-(6)-(7) } \\
& \text { (1)-(2)-(3)-(4)-(5)-(6)-(7) } \\
& \text { (1)-(2)-(3)-(4)-(5)-(6)-(7) } \\
& \text { (1)-(2)-(3)-(4)-(5)-(6)-(7) } \\
& \text { (1)-(2)-(3)-(4)-(5)-(6)-(7) } \\
& \text { (1)-(2)-(3)-(4)-(5)-(6)-(7) } \\
& \text { (1)-(2)-(3)-(4)-(5)-(6)-(7) } \\
& \text { (1)-(2)-(3)-(4)-(5)-(6)-(7) }
\end{aligned}
$$$$
\text { (1)-(2)-(3)-(4)-(5)-(6)-(7) }
$$$$
\text { (1)-(2)-(3)-(4)-(5)-(6)-(7) }
$$$$
\text { (1)-(2)-(3)-(4)-(5)-(6)-(7) }
$$$$
\text { (1)-(2)-(3)-(4)-(5)-(6)-(7) }
$$ 
18. Wenn ich mit anderen zusammenarbeite,

$\begin{array}{cc}\text { trifft } & \text { trifft } \\ \text { gar nicht zu } & \text { vollständig zu }\end{array}$
übernehme ich gewöhnlich die Initiative.

(1)-(2)-(3)-(4)-(5)-(6)-(7)

19. Meine Bekannten würden es als typisch für mich ansehen, dass ich mich durch alle

(1)-(2)-(3)-(4)-(5)-(6)-(7) Schwierigkeiten durchbeiße.

20. Ich empfinde Befriedigung über intensive, konzentrierte Arbeit.

21. Ich arbeite gern an Aufgaben, die ein hohes Maß an Geschick erfordern.

22. Es bereitet mir Freude, mich ganz in eine Aufgabe zu vertiefen.

23. Es ist für mich ein beruflicher Ansporn, einmal eine wichtige Stellung zu erreichen.

24. Ich glaube, dass ich mich beruflich mehr anstrenge als die meisten meiner Kollegen.

25. Wenn ich mit anderen zusammenarbeite, nehme ich die Sachen gern selbst in die Hand.

26. Ich eigne mir lieber neue Kenntnisse an, als mich mit Dingen zu beschäftigen, die ich schon beherrsche.

27. Wenn ich etwas erreicht habe, lag das vor allem an meinem Geschick und meinen Fähigkeiten.

28. Durch eine schwierige Aufgabe fühle ich mich besonders herausgefordert.

29. Wenn ein Risiko besteht, eine Aufgabe nicht zu schaffen, gebe ich mir ganz besondere Mühe.

30. Es ist mir wichtig, meine Tüchtigkeit zu steigern.

(1)-(2)-(3)-(4)-(5)-(6)-(7)

(1)-(2)-(3)-(4)-(5)-(6)-(7)

(1)-(2)-(3)-(4)-(5)-(6)-(7)

(1)-(2)-(3)-(4)-(5)-(6)-(7)

(1)-(2)-(3)-(4)-(5)-(6)-(7)

(1)-(2)-(3)-(4)-(5)-(6)-(7)

(1)-(2)-(3)-(4)-(5)-(6)-(7)

(1)-(2)-(3)-(4)-(5)-(6)-(7)

(1)-(2)-(3)-(4)-(5)-(6)-(7)

(1)-(2)-(3)-(4)-(5)-(6)-(7)

(1)-(2)-(3)-(4)-(5)-(6)-(7)

Anmerkungen. Die Items 2, 8, 11 und 17 entstammen der Skala „Erfolgszuversicht“ aus der Langversion des Leistungsmotivationsinventars (LMI). 


\section{Anhang N: Die Skalen Lernziele (N1) und Arbeitsver- meidung (N2) aus dem SELLMO, Spinath et al. (2002)}

N 1

In der Schule geht es mir darum, ...

\begin{tabular}{|c|c|c|c|c|c|c|}
\hline & & $\begin{array}{l}\text { stimm } \\
\text { gar } \\
\text { nicht }\end{array}$ & $\begin{array}{l}\text { stimmt } \\
\text { eher } \\
\text { nicht }\end{array}$ & $\begin{array}{l}\text { weder/ } \\
\text { noch }\end{array}$ & $\begin{array}{l}\text { stimmt } \\
\text { eher }\end{array}$ & $\begin{array}{l}\text { stimmt } \\
\text { genau }\end{array}$ \\
\hline 1) & ... neue Ideen zu bekommen. & $\square$ & $\square$ & $\square$ & $\square$ & $\square$ \\
\hline 2) & ... etwas Interessantes zu lernen. & $\square$ & $\square$ & $\square$ & $\square$ & $\square$ \\
\hline 3) & $\begin{array}{l}\text {... später knifflige Probleme lösen zu } \\
\text { können. }\end{array}$ & $\square$ & $\square$ & $\square$ & $\square$ & $\square$ \\
\hline 4) & ... komplizierte Inhalte zu verstehen. & $\square$ & $\square$ & $\square$ & $\square$ & $\square$ \\
\hline 5) & $\begin{array}{l}\text {... dass das Gelernte für mich einen Sinn } \\
\text { ergibt. }\end{array}$ & $\square$ & $\square$ & $\square$ & $\square$ & $\square$ \\
\hline 6) & ... zum Nachdenken angeregt zu werden. & $\square$ & $\square$ & $\square$ & $\square$ & $\square$ \\
\hline 7) & ... so viel wie möglich zu lernen. & $\square$ & $\square$ & $\square$ & $\square$ & $\square$ \\
\hline 8) & $\begin{array}{l}\text {... ein tiefes Verständnis für die Inhalte zu } \\
\text { erwerben. }\end{array}$ & $\square$ & $\square$ & $\square$ & $\square$ & $\square$ \\
\hline
\end{tabular}

\section{N 2}

In der Schule geht es mir darum, ...

$$
\begin{array}{ll}
\text { stimmt } & \text { stimmt } \\
\text { gar eher weder/ stimmt stimmt }
\end{array}
$$
nicht nicht noch eher genau

1) ... keine schwierigen Tests oder Arbeiten zu haben.

2) $\quad . . \mathrm{zu}$ Hause keine Arbeiten erledigen $\mathrm{zu}$ müssen.

3) ... keine schwierigen Fragen oder Aufgaben lösen zu müssen.

4) ... nicht so schwer zu arbeiten.

5) ... dass die Arbeit leicht ist.

6) ... aufwendige Aufgaben nicht selber erledigen zu müssen.

7) ... mit wenig Arbeit durch die Schule zu kommen.

$\begin{array}{lllll}\square & \square & \square & \square & \square\end{array}$

8) ... den Arbeitsaufwand stets gering zu halten. 


\section{Anhang O: Selbststeuerungsinventar-Kurzform (SSI-K- 32), Rheinberg \& Wendland (2003)}

\section{Selbstkontrolle und Zielverfolgung}

01. Die meisten Dinge, die ich mir vornehme, setze ich auch um.

09. Auch wenn Probleme auftauchen, bin ich mir meist sicher, dass es irgendwie klappt.

10. Meist ist mir klar, dass das, was ich tue, auch das ist, was ich selber will.

12. Ich habe mich selbst ganz gut im Griff.

13. Dinge, die ich noch nicht erledigt habe, mache ich mir immer wieder bewusst.

17. Auch in schwierigen Situationen vertraue ich drauf, dass ich die Probleme irgendwie bewältigen werde.

23. Bei meinen Handlungen spüre ich meist, dass ich es bin, der so handeln will.

26. Wenn ich etwas erreichen will, setze ich mir ein ganz genaues Ziel.

28. Ich verfolge meist sehr genaue Ziele.

31. Wenn ich mir ein Ziel gesetzt habe, lasse ich mich nicht leicht davon abbringen.

32. Wenn Schwierigkeiten auftauchen, kann ich eine enorme Hartnäckigkeit entwickeln.

\section{Energiemangel und geringe Konzentration}

02. Meine Gedanken schweifen oft von der Sache ab, auf die ich mich eigentlich konzentrieren möchte.

04. Nach unangenehmen Erlebnissen kann ich Gedanken nicht loswerden, die mir die Energie nehmen.

16. Ich fühle mich oft lustlos.

19. Ich habe oft zu wenig Energie.

21. Wenn ich ein Ziel nicht erreiche, verliere ich oft jeden Schwung

22. Oft muss ich an Dinge denken, die mir dem, was ich gerade tue, gar nichts zu tun haben.

27. Ich habe oft ein Ziel, ohne zu wissen, wie ich es erreichen kann.

30. Ich lasse die Dinge lieber auf mich zukommen als genau zu planen.

Trifft

nicht zu

Trifft

$\mathrm{zu}$

$\begin{array}{lllll}2- & 1- & 0 & 1+ & 2+ \\ 2- & 1- & 0 & 1+ & 2+ \\ 2- & 1- & 0 & 1+ & 2+ \\ 2- & 1- & 0 & 1+ & 2+ \\ 2- & 1- & 0 & 1+ & 2+ \\ 2- & 1- & 0 & 1+ & 2+ \\ 2- & 1- & 0 & 1+ & 2+ \\ 2- & 1- & 0 & 1+ & 2+ \\ 2- & 1- & 0 & 1+ & 2+ \\ 2- & 1- & 0 & 1+ & 2+ \\ 2- & 1- & 0 & 1+ & 2+\end{array}$

\section{Selbstmotivierung und Emotionskontrolle}

03. Ich kann ganz gezielt an heitere Dinge denken, um lockerer voranzukommen.

06. Ich kann es schaffen, einer anfangs unangenehmen Tätigkeit zunehmend angenehme Seiten abzugewinnen.

14. Ich kann meine Stimmung so verändern, dass mir dann alles leichter von der Hand geht.

15. Ich erreiche meine beste Form erst dann, wenn Schwierigkeiten auftauchen.

18. Wenn eine Sachelangweilig wird, weiß ich meist, wie ich wieder Spaß daran finden kann.

20. Sobald Hindernisse auftauchen, spüre ich, wie ich aktiver werde.

29. Bei schwierigen Aufgaben kann ich eine enorme Ausdauer entwickeln.

$\begin{array}{ccccc}2- & 1- & 0 & 1+ & 2+ \\ 2- & 1- & 0 & 1+ & 2+ \\ 2- & 1- & 0 & 1+ & 2+ \\ 2- & 1- & 0 & 1+ & 2+ \\ 2- & 1- & 0 & 1+ & 2+ \\ 2- & 1- & 0 & 1+ & 2+ \\ 2- & 1- & 0 & 1+ & 2+ \\ 2- & 1- & 0 & 1+ & 2+\end{array}$

\section{Procastination / Aufschieben}

05. Wenn etwas getan werden muss, beginne ich damit, ohne zu zögern. (-)

07. Ich schiebe viele Dinge vor mir her.

08. Unangenehmes erledige ich oft erst in letzter Minute.

11. Wenn eine Aufgabe erledigt werden muss, packe ich sie am liebsten sofort an. (-)

24. Wenn eine Versuchung auftaucht, kann ich schlecht nein sagen.

25. Ich schiebe unangenehme Dinge oft auf.

$\begin{array}{lllll}2- & 1- & 0 & 1+ & 2+ \\ 2- & 1- & 0 & 1+ & 2+ \\ 2- & 1- & 0 & 1+ & 2+ \\ 2- & 1- & 0 & 1+ & 2+ \\ 2- & 1- & 0 & 1+ & 2+ \\ 2- & 1- & 0 & 1+ & 2+ \\ 2- & 1- & 0 & 1+ & 2+ \\ & & & & \\ 2- & 1- & 0 & 1+ & 2+ \\ 2- & 1- & 0 & 1+ & 2+ \\ 2- & 1- & 0 & 1+ & 2+ \\ 2- & 1- & 0 & 1+ & 2+ \\ 2- & 1- & 0 & 1+ & 2+ \\ 2- & 1- & 0 & 1+ & 2+\end{array}$




\section{Anhang P: Bedeutsame Korrelationen der Skala Elaborationsstrategien (Pisa-Fragebogen) mit anderen Skalen}

Tabelle 18: Korrelation der Skala Elaborationsstrategien mit anderen im Diagnoseinstrumentarium enthaltenen Skalen, $(\mathrm{N}=90)$

\begin{tabular}{|l|c|}
\hline & Skala Elaborationsstrategien \\
\hline LMI-K: Lernbereitschaft & $.45^{* *}$ \\
\hline $\begin{array}{l}\text { LMI-K: Schwierigkeitspräferenz (Items } \\
\text { 31,43,47) }\end{array}$ & $.33^{* *}$ \\
\hline $\begin{array}{l}\text { SSI-K-32: } \\
\text { Selbstmotivierung/Emotionskontrolle }\end{array}$ & $.29 * *$ \\
\hline SELLMO: Lernziele & $.28^{* *}$ \\
\hline PISA: Selbstwirksamkeitserwartungen & $.27 * *$ \\
\hline PISA: Kontrollstrategien & $.32 * *$ \\
\hline $\begin{array}{l}\text { WIRKSCHUL (schulische } \\
\text { Selbstwirksamkeitserwartungen) }\end{array}$ & $.28^{* *}$ \\
\hline
\end{tabular}




\title{
Anhang Q: Vorhersagegleichungen für die relevantesten Kriterien „Eignungsurteil der Lehrer“" sowie „durch- schnittliche Halbjahresnote“6
}

\author{
Erstellt anhand der 1. Studie
}

Vorhersage des Validitätskriteriums „Eignungsurteils der Lehrer“:

Eignung $=1.15+0.01 *$ Deutsch: Wortschatz $\%+0.01 *$ Englisch: Übersetzen $\%+0.03 *$ Punktsumme N-Test $2+0.01 *$ Mathe: Funktion \% - 0.14* Elaborationsstrategien (MW) + 0.14* Selbstkonzept Deutsch (MW)

Vorhersage des Validitätskriteriums ,,durchschnittlichen Halbjahresnote“:

Halbjahresnote (Durchschnitt) $=3.44-0.01 *$ Deutsch: Wortschatz $\%+0.20 *$ Elaborationsstrategien (MW) - $0.01 *$ Gesamtleistungswert

Optimiert anhand der 2. Studie

Vorhersage des Validitätskriterium „Eignungsurteils der Lehrer“:

Eignung $=1.255+0.009 *$ Mathe: Gesamt $\%+0.009 *$ Englisch: Gesamt $\%+0.12 *$ Selbstkonzept Deutsch $-0.117 *$ Elaborationsstrategien $+0.023 *$ N-Test $2+0.004 *$ Deutsch: Wortschatz\%

Vorhersage des Validitätskriteriums „,durchschnittlichen Halbjahresnote“:

Halbjahresnote $($ Durchschnitt $)=3.925-0.009 *$ Deutsch: Wortschatz \% $+0.125 *$ Elaborationsstrategien (MW) $-0.01 *$ Gesamtleistungswert $-0.09 *$ Selbstkonzept Deutsch

Deutsch: Wortschatz\%: erzielter Wert für den Deutsch-Untertest Wortschatz in Prozent Englisch: Übersetzen\%: erzielter Wert für den Englisch-Untertest Übersetzen in Prozent Englisch: Gesamt\%: erzielter Wert für den Englisch-Gesamtwert in Prozent

Mathematik: Funktion\%: erzielter Wert für den Mathematik-Untertest Funktion in Prozent Mathematik: Gesamt\%: erzielter Wert für den Mathematik-Gesamtwert in Prozent Gesamtleistungswert: Durchschnitt der erreichten Prozentwerte der Fächer Mathematik, Deutsch, Englisch

Elaborationsstrategien: Mittelwert der Skala Elaborationsstrategien (Pisa-Fragebogen zu Selbstreguliertem Lernen)

Selbstkonzept Deutsch: Mittelwert der Skala Selbstkonzept Deutsch (DISK-Gitter) 


\section{Anhang R: Prädiktorvariablen und die dazugehörigen konreten Prädiktorwerte des Diagnoseinstrumentariums}

Tabelle 19: Übersicht über die im Diagnoseinstrumentarium enthaltenen Prädiktorwerte.

\begin{tabular}{|c|c|c|}
\hline Bereich & Fragebogen/Test & $\begin{array}{l}\text { Prädiktorwert, der in } \\
\text { die Analysen einging }\end{array}$ \\
\hline \multirow[t]{7}{*}{$\begin{array}{l}\text { KOGNITIVE } \\
\text { DETERMINANTEN }\end{array}$} & \multirow[b]{2}{*}{ Leistungstest Mathematik } & $\begin{array}{l}1 \text { Gesamtwert Mathematik } \\
\text { (über alle } 4 \text { Untertests) in } \\
\text { Prozent }\end{array}$ \\
\hline & & $\begin{array}{l}\text { - } 4 \text { Werte für die Untertests } \\
\text { Größenvergleich, Gleichung, } \\
\text { Zinsrechnung, Funktion in } \\
\text { Prozent }\end{array}$ \\
\hline & \multirow[b]{2}{*}{ Leistungstest Deutsch } & $\begin{array}{l}\text { - } 1 \text { Gesamtwert Deutsch (über } \\
\text { alle } 4 \text { Untertests) in Prozent }\end{array}$ \\
\hline & & $\begin{array}{l}\text { - } 4 \text { Werte für die Untertests } \\
\text { Textverständnis, Wortschatz } \\
\text { und Rechtschreibung in } \\
\text { Prozent }\end{array}$ \\
\hline & \multirow{2}{*}{ Leistungstest Englisch } & $\begin{array}{l}1 \text { Gesamttestwert Englisch } \\
\text { (über alle } 3 \text { Untertests) in } \\
\text { Prozent }\end{array}$ \\
\hline & & $\begin{array}{l}\text { - } 3 \text { Werte für die Untertests } \\
\text { Einsetzen, Matching und } \\
\text { Übersetzen in Prozent }\end{array}$ \\
\hline & \multicolumn{2}{|c|}{$\begin{array}{l}\text { - } 1 \text { Gesamtleistungswert über die drei Gesamtwerte der } \\
\text { Leistungstests Mathematik, Deutsch und Englisch in } \\
\text { Prozent }\end{array}$} \\
\hline \multirow[t]{2}{*}{ Intelligenz } & $\begin{array}{l}\text { Kognitiver Fähigkeitstest: } \\
\text { Figurenklassifikation } \\
\text { (N-Test 1) }\end{array}$ & $\begin{array}{l}\text { - Gesamtsummenwert der } \\
\text { erzielten richtigen Antworten }\end{array}$ \\
\hline & $\begin{array}{l}\text { Kognitiver Fähigkeitstest: } \\
\text { Figurenanalogien } \\
\text { (N-Test 2) }\end{array}$ & $\begin{array}{l}\text { - Gesamtsummenwert der } \\
\text { erzielten richtigen Antworten }\end{array}$ \\
\hline Konzentration & $\begin{array}{l}\text { Aufmerksamkeits- und } \\
\text { Belastungstest } \mathrm{d} 2\end{array}$ & - Konzentrationsleistungswert \\
\hline
\end{tabular}




\begin{tabular}{|c|c|c|}
\hline \multirow{2}{*}{$\begin{array}{l}\text { MOTIVATIONALE } \\
\text { DETERMINANTEN }\end{array}$} & \multirow{2}{*}{$\begin{array}{l}\text { Leistungsmotivationsinventar } \\
\text { Kurzversion (LMI-K) }\end{array}$} & - Gesamtwert (MW) \\
\hline & & $\begin{array}{l}\text { - Skala: Erfolgszuversicht } \\
\text { (MW) }\end{array}$ \\
\hline \multirow[t]{3}{*}{ Leistungsmotivation } & \multirow{3}{*}{$\begin{array}{l}\text { Skalen zur Erfassung der } \\
\text { Lern- und } \\
\text { Leistungsmotivation } \\
\text { (SELLMO) }\end{array}$} & - Skala: Lernziele (MW) \\
\hline & & \\
\hline & & $\begin{array}{l}\text { - Skala: Arbeitsvermeidung } \\
(\mathrm{MW})\end{array}$ \\
\hline \multirow[t]{3}{*}{$\begin{array}{l}\text { MOTIVATIONSSTEUERNDE } \\
\text { KOGNITIONEN }\end{array}$} & \multirow{3}{*}{$\begin{array}{l}\text { Verfahren zur Erfassung des } \\
\text { differentiellen Selbstkonzepts } \\
\text { schulischer Leistungen und } \\
\text { Fähigkeiten (DISK-Gitter) }\end{array}$} & $\begin{array}{l}\text { - Skala: Selbstkonzept Deutsch } \\
\text { (MW) }\end{array}$ \\
\hline & & $\begin{array}{l}\text { - Skala: Selbstkonzept } \\
\text { Englisch (MW) }\end{array}$ \\
\hline & & $\begin{array}{l}\text { - Skala: Selbstkonzept } \\
\text { Mathematik (MW) }\end{array}$ \\
\hline Selbstwirksamkeit & $\begin{array}{l}\text { WIRKSCHUL: Skala zur } \\
\text { schulischen Selbstwirk- } \\
\text { samkeitserwartung }\end{array}$ & - Skala: Wirkschul (MW) \\
\hline \multirow[t]{9}{*}{$\begin{array}{l}\text { SELBSTREGULIERTES } \\
\text { LERNEN }\end{array}$} & \multirow{9}{*}{$\begin{array}{l}\text { Pisa-Fragebogen zum selbst- } \\
\text { regulierten Lernen mit den } \\
\text { Skalen: }\end{array}$} & $\begin{array}{ll}\text { - Skala: Elaborationsstrategien } \\
(\mathrm{MW})\end{array}$ \\
\hline & & $\begin{array}{ll}\text { - Skala: } \\
\text { Wiederholungsstrategien } \\
\text { (MW) }\end{array}$ \\
\hline & & $\begin{array}{l}\text { - Skala: Kontrollstrategien } \\
(\mathrm{MW})\end{array}$ \\
\hline & & $\begin{array}{l}\text { - Skala: Kontrollerwartungen } \\
\text { (MW) }\end{array}$ \\
\hline & & $\begin{array}{l}\text { - Skala: Selbstwirksamkeits- } \\
\text { erwartungen (MW) }\end{array}$ \\
\hline & & - Skala: Interesse Lesen (MW) \\
\hline & & $\begin{array}{ll}\text { - Skala: Interesse Mathematik } \\
\text { (MW) }\end{array}$ \\
\hline & & $\begin{array}{ll}\text { - Skala: Instrumentelle } \\
\text { Motivation (MW) }\end{array}$ \\
\hline & & $\begin{array}{l}\text { - Skala: Anstrengung und } \\
\text { Ausdauer (MW) }\end{array}$ \\
\hline
\end{tabular}




\begin{tabular}{|c|c|c|}
\hline \multirow[t]{5}{*}{$\begin{array}{l}\text { SELBSTREGULIERTES } \\
\text { LERNEN }\end{array}$} & \multirow{5}{*}{$\begin{array}{l}\text { Selbststeuerungsinventar- } \\
\text { Kurzversion (SSI-K-32) } \\
\text { bestehend aus den Skalen }\end{array}$} & $\begin{array}{ll}\text { - Skala: Selbstkontrolle u. } \\
\text { Zielverfolgung (MW) }\end{array}$ \\
\hline & & $\begin{array}{l}\text { - Skala: Engergiemangel u. } \\
\text { geringe Konzentration (MW) }\end{array}$ \\
\hline & & $\begin{array}{l}\text { - Skala: Selbstmotivierung u. } \\
\text { Emotionskontrolle (MW) }\end{array}$ \\
\hline & & $\begin{array}{l}\text { - Skala: Procrastination u. } \\
\text { Aufschieben }\end{array}$ \\
\hline & & - Gesamtwert (MW) \\
\hline
\end{tabular}

Anmerkung. Die Abkürzung „MW“ steht für Mittelwert. 


\section{LEBENSLAUF}

\section{Persönliche Daten}

Name:

Dittmar

Vorname:

Maike

Geburtsdatum

20.10.1976

Geburtsort:

Naumburg (Saale)

Staatsangehörigkeit: deutsch

\section{WISSENSCHAFTLICHER WERDEGANG}

10/1996 - 9/1998 Grundstudium der Psychologie an der Martin-LutherUniversität Halle-Wittenberg in Halle

10/1998 - 9/1999 Auslandsstudium der Psychologie an der Université de Caen in Frankreich im Rahmen des Erasmus Programms

10/1999 - 1/2004 Hauptstudium der Psychologie an der Georg-AugustUniversität Göttingen mit den Schwerpunkten Klinische Psychologie und Pädagogische Psychologie

10/2001 - 03/2003 Studentische Hilfskraft in der Abteilung für Pädagogische Psychologie und Entwicklungspsychologie der Universität Göttingen

seit $01 / 2004$

Geprüfte wissenschaftliche Hilfskraft/ wissenschaftliche Mitarbeiterin in der Abteilung für Pädagogische Psychologie und Entwicklungspsychologie der Universität Göttingen mit dem Ziel der Promotion 